\title{
Complex Bounds for Real Maps
}

\author{
Trevor Clark, Sebastian van Strien, Sofia Trejo \\ Imperial College London, London, UK. \\ E-mail: trevorcclark@gmail.com; s.van-strien@imperial.ac.uk; sofia.trejo.a@gmail.com
}

Received: 1 November 2013 / Accepted: 22 May 2017

Published online: 12 August 2017 - (C) The Author(s) 2017. This article is an open access publication

\begin{abstract}
In this paper we prove complex bounds, also referred to as a priori bounds for $C^{3}$, and, in particular, for analytic maps of the interval. Any $C^{3}$ mapping of the interval has an asymptotically holomorphic extension to a neighbourhood of the interval. We associate to such a map, a complex box mapping, which provides a kind of Markov structure for the dynamics. Moreover, we prove universal geometric bounds on the shape of the domains and on the moduli between components of the range and domain. Such bounds show that the first return maps to these domains are well controlled, and consequently such bounds form one of the corner stones in many recent results in one-dimensional dynamics, for example: renormalization theory, rigidity, density of hyperbolicity, and local connectivity of Julia sets.
\end{abstract}

\section{Contents}

1. Introduction and Statement of the Main Results . . . . . . . . . . . . . . . 1002

2. The Generalized Enhanced Nest . . . . . . . . . . . . . . . . . . . . . . . 1019

3. Real Bounds Associated to the Enhanced Nest . . . . . . . . . . . . . . . 1024

4. Poincaré Disks and Tools for Dealing with Analytic Maps . . . . . . . . . 1043

5. Tools for Dealing with $C^{3}$ Maps . . . . . . . . . . . . . . . . . . . . . . . 1049

6. Pulling Back a Poincaré Disc Through High Iterates of First Return Maps . 1056

7. Pulling Back a Poincaré Disc Along a Chain with Bounded Combinatorial Depth . . . . . . . . . . . . . . . . . . . . . 1067

8. Pulling Back a Poincaré Disc One Step Along the Enhanced Nest . . . . . 1070

9. Extension to a QR Quasi-Box Mapping . . . . . . . . . . . . . . . . . . . 1089

10. Box Mappings and Complex Bounds . . . . . . . . . . . . . . . . . . . . 1098

11. Table of Notation and Terminology . . . . . . . . . . . . . . . . . . 1116

References ....................... . . 1117 


\section{Introduction and Statement of the Main Results}

The purpose of this paper is to develop a unified technique that allows one to treat a real analytic or even a $C^{3}$ map of the interval as a complex dynamical system, where the domain and range provide a Markov-like structure for the dynamics. This problem has a long history, which we will discuss later. Our results are new for analytic maps, and, in some cases, even when the mapping is a polynomial.

If the interval mapping is real analytic, the associated complex mapping is holomorphic, and when the mapping is only smooth, the complex extension is a quasiregular mapping that is asymptotically holomorphic on its real trace. Crucially, we will obtain geometric bounds (usually referred to as complex bounds or a priori bounds) for this complex extension. In Sect. 1.6 we will explain why complex bounds are useful. The following informal statement, which we will make more precise in the next subsection, summarizes our results:

Main Theorem (Informal Statement). Let $M \subset \mathbb{R}$ be a compact interval. Assume that $f: M \rightarrow M$ is a real analytic map (respectively a $C^{3}$ map with critical points of integer orders) with no critical points on $\partial M$, and a critical point $c$ such that $f$ is persistently recurrent on $\omega(c)$. Then there exist arbitrarily small, combinatorially defined neighbourhoods $\hat{I} \subset M$ of $\operatorname{Crit}(f) \cap \omega(c)$ such that we can associate to the (real) first return map to $\hat{I}$ a complex box mapping (respectively a quasiregular box mapping) with complex bounds.

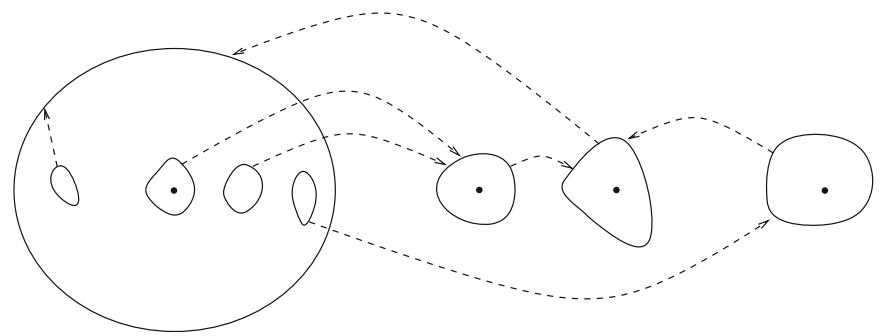

The notion persistently recurrent on $\omega(c)$ is defined on p. 20.

Return maps which are not persistently recurrent on $\omega(c)$ are much easier to work with, and complex box mappings will be constructed for such maps in [CvS].

Definition 1.1 (Complex box mapping). A mapping $F: \mathcal{U} \rightarrow \mathcal{V}$ is a complex box mapping if $F$ is holomorphic and $\mathcal{U} \subset \mathcal{V}$ are open subsets of the complex plane where

- $\mathcal{V}$ is a union of finitely many pairwise disjoint Jordan disks;

- every connected component $V$ of $\mathcal{V}$ is either a connected component of $\mathcal{U}$ or $V \cap \mathcal{U}$ is a union of Jordan disks with pairwise disjoint closures that are compactly contained in $V$;

- for each component $U$ of $\mathcal{U}, F(U)$ is a component of $\mathcal{V}$ and $F \mid U$ is a proper map;

We say that $F: \mathcal{U} \rightarrow \mathcal{V}$ is a quasiregular complex box mapping, abbreviated qr box mapping, if $F$ is a quasiregular mapping that satisfies the remaining conditions of the definition of a complex box mapping, see Sect. 5.3. When it will not cause confusion, we will refer to qr box mappings simply as box mappings. As usual, a polynomial-like mapping is a holomorphic, proper mapping $F: U \rightarrow V$ between topological disks $U \Subset V \subset \mathbb{C}$. We call a mapping $F: U \rightarrow V$, a qr polynomial-like map if $U \Subset V$ are 
topological disks in $\mathbb{C}$ and $F: U \rightarrow V$ is a proper mapping that can be expressed as $F=P \circ h$ where $h: U \rightarrow U$ is quasiconformal and $P: U \rightarrow V$ is holomorphic.

A complex box mapping (or a qr box mapping) $F: \mathcal{U} \rightarrow \mathcal{V}$ is called real-symmetric if $\mathcal{U}$ and $\mathcal{V}$ are both real-symmetric, and $\overline{F(\bar{z})}=F(z)$. The box mappings constructed in this paper are real-symmetric, and indeed every construction in this paper is carried out real-symmetrically.

We say that the map $F: \mathcal{U} \rightarrow \mathcal{V}$ has complex bounds (also referred to as a priori bounds) if one has estimates on the geometry of $\mathcal{U}$ and $\mathcal{V}$, see Sect. 1.1.2.

Throughout this paper, we only consider first return maps restricted to the components of their domains that intersect $\omega(c)$, for a critical point $c$, and we will implicitly assume this throughout.

Since $f$ is persistently recurrent on $\omega(c), \omega(c)$ is a minimal set for $f$, and hence it is compact. Thus, because each component of $\mathcal{U}$ intersects $\omega(c), \mathcal{U}$ has at most finitely many components. Complex box mappings whose domains contain only finitely many components are also known as generalized polynomial-like maps.

1.1. Some terminology. Before we can state the main results of this paper, we need some terminology.

1.1.1. First return and landing maps. Consider a complex box mapping $F: \mathcal{U} \rightarrow \mathcal{V}$. We will say that $P$ is a (complex) puzzle piece if $P$ is a component of $F^{-n}(V)$, where $V$ is any connected component of $\mathcal{V}$ and $n \geq 0$. To a puzzle piece, we associate three mappings: the (first) return mapping, the (first) landing map and the (first) entry map. Let

$$
\operatorname{Dom}(P)=\left\{z \in \mathcal{U}: F^{k}(z) \in P \text { for some } k \in \mathbb{N}\right\},
$$

where we take $\mathbb{N}=\{1,2,3, \ldots\}$. For any $z \in \operatorname{Dom}(P)$, let $k(z) \in \mathbb{N}$ be minimal so that $F^{k(z)}(z) \in P$. The first entry mapping to $P$ is the mapping from $\operatorname{Dom}(P)$ to $P$ defined by $z \mapsto F^{k(z)}(z)$. The first landing map to $P$ is defined by $z \mapsto F^{k(z)}(z)$ for $z \in \operatorname{Dom}(P) \backslash P$ and by the identity on $P$. We define the first return mapping to $P$ as the restriction of the first entry mapping to $P$ :

$$
R_{P}: \operatorname{Dom}(P) \cap P \rightarrow P, \text { where } R_{P}(z)=F^{k(z)}(z) .
$$

Suppose that $z \in \operatorname{Dom}(P)$. The connected component of $\operatorname{Dom}(P)$ that contains $z$ is denoted by $\mathcal{L}_{z}(P)$ and will be called a first entry domain to $P$ that contains $z$. We will also call $\mathcal{L}_{z}(P)$ a return domain to $P$, if $z \in \operatorname{Dom}(P) \cap P$. We define the first landing domain to $P$ containing $z$ by

$$
\hat{\mathcal{L}}_{z}(P)=\left\{\begin{aligned}
P & \text { if } z \in P \\
\mathcal{L}_{z}(P) & \text { if } z \notin P .
\end{aligned}\right.
$$

We will also use these definitions in the real case where $P \subset M$ is an interval.

1.1.2. Complex bounds ( $\rho$-nice, $\rho$-free, $\rho$-bounded geometry). Let $\rho>0$. A puzzle piece $P$ is called $\rho$-nice if for any $x \in P \cap \omega(c)$ one has $\bmod \left(P \backslash \mathcal{L}_{x}(P)\right) \geq \rho$, and $\rho$-free if there are puzzle pieces $P^{+} \supset P \supset P^{-}$such that $\left(P^{+} \backslash P^{-}\right) \cap \omega(c)=\emptyset$, $\bmod \left(P^{+} \backslash P\right) \geq \rho$ and $\bmod \left(P \backslash P^{-}\right) \geq \rho$. We refer to the annulus $P^{+} \backslash P^{-}$, which is disjoint from $\omega(c)$, as free space. We say that a simply connected domain $U$ has $\rho$ bounded geometry with respect to $x \in U$ if the Euclidian ball $B(x, \rho \cdot \operatorname{diam}(U)) \subset U$. 
A domain $U$ is said to have $\rho$-bounded geometry if there is an $x \in U$ such that $U$ has $\rho$-bounded geometry with respect to $x$.

Let $\mathcal{V}$ be the range of a (qr) complex box mapping. We say that $\mathcal{V}$, respectively, is $\rho$-nice, is $\rho$-free or has $\rho$-bounded geometry if $\mathcal{V}$ is the union of puzzle pieces $V$, such that each $V$, respectively, is $\rho$-nice, is $\rho$-free or has $\rho$-bounded geometry.

If there exist $\rho>0$, universal, and a neighbourhood $\mathcal{V}$ of $\operatorname{Crit}(f) \cap \omega(c)$ such that $R \mathcal{V}: \operatorname{Dom}(\mathcal{V}) \cap \mathcal{V} \rightarrow \mathcal{V}$ is a (qr) complex box mapping such that one has that $\mathcal{V}$ is $\rho$-nice and has $\rho$-bounded geometry, then $R \mathcal{V}$ is said to have complex bounds.

1.1.3. Extendible box mappings. If $F: \cup U_{j} \rightarrow \cup V_{i}$ is a complex box mapping with $b$ critical points, we say that $F$ is $v$-extendible if there exists $v>0$ such that for each $i$ there are topological disks $V_{i}^{\prime} \supset V_{i}$ with $\bmod \left(V_{i}^{\prime} \backslash \bar{V}_{i}\right)>v$ such that the following hold:

(1) for each $i$, if $k$ is such that $F\left(V_{i}\right)=V_{k}$, then $F \mid V_{i}$ extends to a branched covering from $V_{i}^{\prime}$ to $V_{k}^{\prime}$ and there are no critical points of $F$ in $V_{i}^{\prime} \backslash V_{i}$;

(2) for each component $U_{j}$ of $\mathcal{U}$, if $k$ is such that $F\left(U_{j}\right)=V_{k}$, then there exists a topological disk $U_{j}^{\prime} \supset U_{j}$ so that $F \mid U_{j}$ extends to a holomorphic map from $U_{j}^{\prime}$ to $V_{k}^{\prime}$ with no additional critical points in $U_{j}^{\prime} \backslash U_{j}$;

(3) If $U_{j} \subset V_{k}$, then $U_{j}^{\prime} \subset V_{k}$.

We will define $v$-extendible for qr box mappings in the remarks following the statement of Theorem 1.2.

The notions of $\delta$-nice and $\delta$-free have real analogues, see p. 24.

1.1.4. Remormalizable maps, periodic, central and terminating intervals. An interval $I$ is a periodic interval of $f: M \rightarrow M$ if there exists $s>1$ such that $I, f(I), \ldots, f^{s-1}(I)$ have pairwise disjoint interiors and $f^{s}(I) \subset I$ with $f^{s}(\partial I) \subset \partial I$. The integer $s$ is called the period of $I$. We say that a map $f$ of the interval is renormalizable at $x$ if it possesses a periodic subinterval containing $x$, and that it is infinitely renormalizable at $x$ if there exist arbitrarily large integers $p>0$ such that $f$ has a periodic subinterval containing $x$ with period $p$.

Suppose that $J$ is an interval and $c \in J$ is a recurrent critical point of $f$. Let $p \geq 1$ be minimal so that $f^{p}(c) \in J$. We say that the return to $J$ is central if $f^{p}(c) \in \mathcal{L}_{c}(J)$. Let $\mathcal{L}_{c}^{0}(J)=J$ and $\mathcal{L}_{c}^{n}(J)=\mathcal{L}_{c}\left(\mathcal{L}_{c}^{n-1}(J)\right)$ for all $n \geq 1$, then we say that $J$ is terminating if the returns of $c$ to $\mathcal{L}_{c}^{n}(J)$ are central for all $n$.

If $J$ is terminating, we define $J^{\infty}=\cap_{n \geq 1} \mathcal{L}_{c}^{n}(J)$; under these circumstances, $J^{\infty}$ is a periodic interval of period $p$, and $f$ is renormalizable at $c$.

Suppose that $\hat{I}$ and $\hat{J}$ are unions of intervals. We say that a mapping $g: \hat{J} \rightarrow \hat{I}$ extends to $G: \mathcal{U} \rightarrow \mathcal{V}$ if for each connected component $J$ of $\hat{J}$, there exists a unique connected component $U$ of $\mathcal{U}$ such that $J \subset U$ and $G|J=g| J$. Moreover, we require that each component $U$ of $\mathcal{U}$ contains a component $J$ of $\hat{J}$, that each component $V$ of $\mathcal{V}$ contains a unique component $I$ of $\hat{I}$, and that the maps $G$ and $g$ have the same critical points.

1.2. Complex bounds in the real analytic case. We can now state our main theorem for analytic maps (see Sect. 2.1 for the definition of a real puzzle piece).

Theorem 1.1. Let $M \subset \mathbb{R}$ be a compact interval. Assume that $f: M \rightarrow M$ is a real analytic map with a critical point $c$ such that $f$ is persistently recurrent on $\omega(c)$. Then there 
exist $\rho>0$ and combinatorially defined intervals (puzzle pieces) I $\ni$ c of arbitrarily small diameter so that the following holds. Let

$$
\hat{I}:=\bigcup_{c^{\prime} \in \operatorname{Crit}(f) \cap \omega(c)} \hat{\mathcal{L}}_{c^{\prime}}(I) .
$$

1. Suppose that $f$ is non-renormalizable. Then the first return map to $\hat{I}$ extends to a complex box mapping

$$
F: \mathcal{U} \rightarrow \mathcal{V} \text { so that } \mathcal{V} \cap \mathbb{R}=\hat{I} \text { and }
$$

- for each component $U$ of $\mathcal{U}, F \mid U$ has at most one critical point,

- each component of $\mathcal{V}$ is $\rho$-nice and $\rho$-free,

- each component of $\mathcal{V}$ has $\rho$-bounded geometry.

2. Suppose that I is a terminating interval for $f$. Then the return map to $I^{\infty}$ extends to a polynomial-like map $F: U \rightarrow V$ such that $\bmod (V \backslash U)>\rho$.

Remarks. (a) If $I$ is sufficiently small, then each component of $\hat{\mathcal{L}}_{c^{\prime}}(I), c^{\prime} \in \operatorname{Crit}(f) \cap$ $\omega(c)$, contains exactly one critical point of $f$, so each component $V$ of $\mathcal{V}$ contains exactly one critical point of $F$.

(b) The intervals $I$ will be obtained from the generalized enhanced nest defined on p. 22. In the non-renormalizable case this nest coincides with the enhanced nest from $[\mathrm{KSvS}]$. In Theorems 10.1 and 10.18 we restate this theorem making the choice of $I$ explicit.

(c) When $f$ is infinitely renormalizable, then the assertion holds for any periodic interval $I$ which is sufficiently small. If $f$ is at most finitely renormalizable, then as in the non-renormalizable case, $I$ can be taken to be a sufficiently small pullback of some fixed interval $I_{0}$.

(d) The number $\rho$ is universal, or beau, (a universal bound that holds eventually) in the following sense: there exists $\varepsilon>0$, which may depend on $f$, such that whenever the combinatorially defined interval $I$ has $|I|<\varepsilon$, Theorem 1.1 holds with $\rho>0$ dependant only on the number of critical points in $\omega(c)$ and their orders, and not on $f$. (It is crucial that we take $I$ to be sufficiently small depending on $f$. To get beau bounds, we use the real bounds from Sect. 3 and an argument similar to [ $\mathrm{dMvS}$, Theorem IV.B] which states that there exists a beau $\epsilon>0$ so that for each $f$ there exists $N$ so that each periodic orbit of period at least $N$ has multiplier $\geq 1+\epsilon$.)

(e) The reason that we do not include $\delta$-free in the definition of complex bounds is that we require that the free space be defined by puzzle pieces, and in the infinitely renormalizable case, we do not prove this. However, in any case, the mapping $F$ is $\rho$-extendible.

The terminology 'beau bounds' was introduced by Dennis Sullivan. The underlying concept is a crucial property for results on renormalization, see Sect. 1.6.

1.3. Complex bounds in the $C^{3}$ setting. Let us now explicitly state our main result for $C^{3}$ interval mappings. Suppose that $c \in \operatorname{Crit}(f)$ is a persistently recurrent critical point. Let $c_{0} \in \omega(c) \cap \operatorname{Crit}(f)$ be of even order, if there is such a critical point in $\omega(c)$, and otherwise choose $c_{0}$ arbitrarily. We let $I_{0} \supset I_{1} \supset I_{2} \supset \cdots$ denote the enhanced nest about $c_{0}$. See Sect. 2 for the definition of this nest. Whenever $f$ is at most finitely 
renormalizable at $c_{0}$, we assume that $I_{0}$ is contained in the smallest periodic interval for $f$ containing $c_{0}$. If $I \subset \mathbb{R}$ is an interval we let $D_{\theta}(I)$ the Poincaré disk with angle $\theta$ with real trace $I$, see p. 17 for the definition.

Theorem 1.2 (Complex bounds in the $C^{3}$ case). Let $M \subset \mathbb{R}$ be a compact interval. Suppose that $f: M \rightarrow M$ is $C^{3}$ and its critical points are of integer order (i.e. $f$ is contained in the class $\mathcal{A}_{b}^{3}$ defined in Sect. 1.10.2). There exists $\rho>0$ and $C>0$, universal (depending only on the number of critical points of $f$ and their order, i.e. on $\underline{b}$, but not on $f$ ), such that for each $n$ sufficiently large the following holds. Suppose that $c_{0}$ is a critical point such that $f$ is persistently recurrent on $\omega\left(c_{0}\right)$.

(1) Suppose that $f$ is finitely renormalizable. Let $I_{0} \supset I_{1} \supset I_{2} \supset \cdots$ be the enhanced nest for $f$ about $c_{0}$ (constructed so that $I_{0}$ is contained in the smallest periodic interval of $f$ that contains $\left.c_{0}\right)$. Let $\hat{I}_{n}=\cup_{c \in \omega\left(c_{0}\right) \cap \operatorname{Crit}(f)} \hat{\mathcal{L}}_{c}\left(I_{n}\right)$. Then for all $n$ sufficiently big, the first return map to $\hat{I}_{n}$ extends to a $\kappa_{n}$-qr box mapping $F: \mathcal{U} \rightarrow \mathcal{V}$ with $\operatorname{Crit}(F)=\operatorname{Crit}(f)$ that is $\rho$-extendible and such that $\mathcal{V} \cap \mathbb{R}=\hat{I}_{n}$; for each component $U$ of $\mathcal{U}, F \mid U$ is at most unicritical; the components of $\mathcal{V}$ are $\rho$-nice, $\rho$ free; the components of $\mathcal{U}$ and $\mathcal{V}$ have $\rho$-bounded geometry. Moreover, there exists $\theta^{\prime} \in(0, \pi)$ such that for each component $U$ of $\mathcal{U}$ or $\mathcal{V}$, there exists an interval $\tilde{I}_{U} \supset(1+2 \rho)(U \cap \mathbb{R})$ such that $U$ is contained in $D_{\theta^{\prime}}\left(\tilde{I}_{U}\right)$.

(2) If $f$ is infinitely renormalizable, then for all s sufficiently big, if $J$ is a periodic interval for $f$ of period $s$, then $f^{s}: J \rightarrow J$ extends to a $\kappa(V)$-qr polynomial-like mapping $F: U \rightarrow V$ with $\operatorname{Crit}(F)=\operatorname{Crit}(f), \bmod (V \backslash U) \geq \rho, U$ has $\rho$-bounded geometry and $\operatorname{diam}(V)<C|J|$.

The number $\kappa_{n}$ depends on $\max _{V} \operatorname{diam}(V)$, where the maximum is taken over the component of $\mathcal{V}, \kappa_{n}$, and $\kappa(V)$ depends only on $\operatorname{diam}(V)$ and both $\kappa_{n}$ and $\kappa(V)$, tend to 1 as the corresponding diameters tend to 0 .

1.4. Complex bounds for induced mappings. We remark that the proof of our results imply the following corollary, which will be useful in applications.

Corollary 1.3. Let $N$ denote the circle or interval. Suppose that $f: N \rightarrow N$ is $C^{3}$ and its critical points are of integer order (i.e. $f$ is contained in the class $\mathcal{A}_{b}^{3}$ defined in Sect. 1.10.2). Assume that $M$ is a union of intervals in $N$ and that $M^{\prime}$ is a union of intervals $J^{\prime} \subset M$ such that for each interval $J^{\prime}$, there exists $k_{J^{\prime}} \in \mathbb{N}$ such that $f^{k_{J^{\prime}}}\left(J^{\prime}\right) \subset M$, and $f^{k_{J^{\prime}}}$ does not have a critical point on $\partial J^{\prime}$. Define $F: M^{\prime} \rightarrow M$ on each interval $J^{\prime} \subset M^{\prime}$ by $F\left|J^{\prime}=f^{k^{\prime}}\right| J^{\prime}$. Assume that $c$ is a critical point of $F$ such that $F$ is persistently recurrent on $\omega(c)$. Then the conclusion of Theorem 1.2 holds for $F$ at $c$.

For example, suppose that $f: S^{1} \rightarrow S^{1}$ is an analytic mapping of the circle that is not injective. Then $f$ has a periodic point $p$, see $[\mathrm{CvS}]$. Let $s$ be the period of $p$, and let $\mathcal{O}=\left\{p, f(p), \ldots, f^{s-1}(p)\right\}$. Then $\mathcal{Y}_{0}=S^{1} \backslash f^{-1}(\mathcal{O})$ is a partition of $S^{1}$ by real puzzle pieces, see Sect. 2.1. Suppose that $f$ has critical point $c_{0}$ such that $f$ is persistently recurrent at $c_{0}$, and let $Y_{0}$ be the component of $\mathcal{Y}_{0}$ that contains $c_{0}$. Then the results of this paper hold for the return mapping to $Y_{0}$ restricted to the components of the domain that intersect $\omega\left(c_{0}\right)$. 
1.5. Previous results on complex bounds. Let us first give some historical background before discussing in the next subsection why complex bounds are crucial for results on renormalization, quasiconformal rigidity and ergodic properties of one-dimensional dynamical systems. Complex bounds were first proved by Sullivan for certain infinitely renormalizable unimodal maps $[\mathrm{Su}]$, see also $[\mathrm{dMvS}]$. They were proved for real unicritical polynomials, in [LvS1,S1,LY] and [GS]. Let us now summarize some of the past work for multimodal analytic maps with all critical points real and of even order:

- Complex bounds were proved for infinitely renormalizable maps with bounded combinatorics in [Sm1].

- In [S3] complex bounds were proved for infinitely renormalizable maps. In addition, for at most finitely renormalizable maps, Shen proves a somewhat weaker version of complex bounds. Namely, the existence of complex box mappings with the property that each domain of an iterate of the box mapping is contained in a Poincare disk with real trace of length comparable to the range of the (real) return map, see [S3, Theorem 3'].

- Complex bounds, analogous to those in this paper, were proved for at most finitely renormalizable real polynomials with all critical points even and real in [KSvS].

Complex bounds for various classes of unicritical analytic maps whose critical point is of odd order, including covering maps of the circle, and certain real polynomial maps and Blaschke products, were obtained in [LvS2]. The methods in that paper, and the corresponding paper [Le1] in which real bounds are proved, do not seem to go through to the case of two or more critical points of odd order. Indeed, also in our proof the presence of odd critical points requires us to overcome significant additional difficulties.

We should note that if $f$ is a non-renormalizable polynomial (not necessarily real) with only hyperbolic periodic orbits in the complex plane, then the construction of a complex box mapping follows immediately from the Yoccoz puzzle construction, see $[\mathrm{KvS}]$. In fact, if there are neutral periodic points which are of parabolic type, this construction can be easily made as well: simply consider rays landing on repelling periodic points in the boundary of one of the petals of the periodic point. However, if $f$ has a non-parabolic neutral orbit then in general it may be impossible to find a complex box mapping or even a periodic point with two rays landing on it. If $f$ is a real polynomial such rays, and therefore a complex box mapping, exists, but nevertheless it seems the only way to obtain a complex box mapping for which each puzzle piece contains at most one critical point is through complex bounds, see [KvS, Sect. 2.2].

If $f$ is at most finitely renormalizable polynomial with only hyperbolic periodic points, then the results in $[\mathrm{KvS}]$ imply that complex bounds indeed hold for $f$. The method there relies on an important lemma by Kahn-Lyubich, see [KL1], and the results in $[\mathrm{KvS}]$ do not require the polynomial to be real (and it does not matter whether the critical points are of even or odd order). Because of this, the proof in [KSvS] can be simplified: one can replace Sects. 8 to 11 in [KSvS] by the more general results derived in $[\mathrm{KvS}]$. However, the methods in $[\mathrm{KvS}]$ do not provide complex bounds when $f$ is infinitely renormalizable or when $f$ is $C^{3}$.

So combining the puzzle construction and the complex bounds from [KvS] shows that if $f$ is a polynomial that is at most finitely renormalizable and has only hyperbolic or parabolic periodic points, then there exists a complex box mapping so that each puzzle piece contains at most one critical point. But if $f$ is a real polynomial which is either infinitely renormalizable (possibly at a non-real critical point) or with a non-parabolic periodic point (and a non-real critical point), then $[\mathrm{KvS}]$ does not provide complex bounds. 
Thus, even for real polynomials our theorem is new: previous results did not establish beau complex bounds for (at most) finitely renormalizable real polynomials with either non-real critical points or real critical points of odd order, and also not for infinitely renormalizable real polynomials with critical points of odd order.

Previous proofs of complex bounds often require dividing the proof into the (essentially) bounded geometry and the big geometry cases. Having big geometry simplifies the construction of a complex box mapping at a single level, but when there are no bounds on the scaling factors, it is difficult to transfer estimates to deeper levels, see the comment before Proposition 10.5.

The purpose of this paper is to treat all situations in a fully unified manner, dealing with non-renormalizable and renormalizable maps, with any combinatorics, through essentially the same inductive framework. The methods we develop allow us to deal with critical points of any (integer) order. The construction builds on the one given in Sects. 8 to 11 of [KSvS], but encompasses the infinitely renormalizable case, and overcomes the issues that arise from the presence of odd critical points. Another important feature of the proof is that it allows one to associate quasiregular box mappings to $C^{3}$ maps, see Theorem 1.2. This is also an important reason why we did not aim for short-cuts in the proof in the setting of real analytic maps, which do not generalize to the setting of maps with asymptotically holomorphic extensions. In this paper we will not discuss complex bounds for smooth homeomorphisms of the circle, but merely refer to [dFdM1, dFdM2, Ya].

For unicritical, (at most) finitely renormalizable complex polynomials without neutral periodic orbits, moduli bounds were proved in [KL2]. We should emphasise that complex bounds do not hold in general for complex maps; there are infinitely renormalizable complex quadratic maps for which complex bounds are known to fail, see for example [Le2]. However, they do hold for unicritical maps with certain combinatorics, see [Ka, KL3, KL4]. For an early work on non-renormalizable mappings with specific combinatorics, where the shape of puzzle pieces was well controlled we draw the readers attention to [Sm3].

1.6. The usefulness of complex bounds: applications. Because of the Koebe Distortion Theorem and the behaviour of the mapping $z \mapsto z^{\ell}$, one immediately sees that complex bounds for $F: \mathcal{U} \rightarrow \mathcal{V}$ give control on the distortion of diffeomorphic mappings onto components of $\mathcal{V}$ and on the shapes of certain puzzle pieces. However, the implications of complex bounds are much deeper. The results in this paper are an important ingredient in results on the topology Julia sets, renormalization, quasiconformal rigidity and ergodic properties.

1.6.1. Topological and ergodic properties. By methods that are by now standard, see for instance $[\mathrm{LvS} 3, \mathrm{~S} 2]$ and $[\mathrm{KvS}]$, the results of this paper imply

Theorem 1.4. Suppose that $f$ is a real polynomial with real critical points. Then the Julia set of $f$ is locally connected, and $f$ supports no measurable invariant line field on its Julia set.

Note that, complex bounds for reluctantly recurrent maps are easier to obtain and the proof can be found in $[\mathrm{CvS}]$.

Before now, in the multicritical case, such a result was only known when all critical points were of even order. In the unicritical case, local connectivity was proved in the presence of one even critical point in $[\mathrm{LvS} 1, \mathrm{LY}, \mathrm{GS}]$ and $[\mathrm{HJ}]$ and for the Julia sets of 
certain Blaschke products with a single critical point on the unit circle in [LvS2]. For multicritical polynomials with all critical points real and of even order, local connectivity was proved for certain infinitely renormalizable maps in [Sm1], in [S3] (in the case of 'bounded geometry') and without assumptions on the geometry in [KSvS]. Absence of invariant line fields was first proved in [M1] and subsequently in [LvS3,LvS2,S2] and $[\mathrm{KvS}]$.

1.6.2. Quasisymmetric rigidity. Complex bounds also play a key role in proving quasisymmetric rigidity. In particular, complex bounds are a crucial hypothesis in the QCCriterion of [KSvS]. The results of this paper immediately extend the results of [KSvS] to include polynomials with odd critical points:

Theorem 1.5. Suppose that $f$ and $\tilde{f}$ are two real polynomials, with real critical points. Assume that $f$ and $\tilde{f}$ are topologically conjugate as dynamical systems on the real line, that corresponding critical points for $f$ and $\tilde{f}$ have the same order and that parabolic points correspond to parabolic points, then $f$ and $\tilde{f}$ are quasiconformally conjugate as dynamical systems on the complex plane.

The results of this paper are a vital ingredient in extending this result for polynomials to all analytic maps and to a broad class of $C^{3}$ mappings.

Theorem 1.6 (Clark-van Strien $[\mathrm{CvS}]$ ). Assume that $f, g:[0,1] \rightarrow \mathbb{R}$ with $f(\{0,1\}) \subset$ $\{0,1\}$ are real analytic and topologically conjugate. Alternatively, assume that $f, g: S^{1} \rightarrow S^{1}$ are topologically conjugate and that $f$ and $g$ each have at least one critical point or at least one periodic point. Moreover, assume that the topologically conjugacy is a bijection between

- the sets of critical points and the orders of corresponding critical points are the same;

- the set of parabolic periodic points.

Then the conjugacy between $f$ and $g$ is quasisymmetric.

For smooth mappings, we have:

Theorem 1.7 (Clark-van Strien $[\mathrm{CvS}]$ ). Suppose that $f, g:[0,1] \rightarrow[0,1]$, or alternatively that $f, g: S^{1} \rightarrow S^{1}$ each has least one critical point or at least one periodic point, are $C^{3}$, each with a finite number of critical points. Suppose that at each $c \in \operatorname{Crit}(f)$, one can locally express $f(x)=[\phi(x)]^{\ell}+f(c)$ where $\phi$ is a $C^{3}$ diffeomorphism with $\phi(c)=0$ and $\ell$ is an integer $\geq 2$, and likewise for $g$. Assume that $f$ and $g$ have only repelling periodic points. Suppose that $f$ and $g$ are topologically conjugate and that the conjugacy is a bijection between $\operatorname{Crit}(f)$ and $\operatorname{Crit}(g)$ and that the orders of corresponding critical points is the same. Then $f$ and $g$ are quasisymmetrically conjugate.

Under some additional smoothness and genericity assumptions, we can remove the condition that all periodic orbits be repelling, as in Theorem 1.6. These theorems extend earlier work for quadratic polynomials [Ly2,GS2,GS3], and for polynomials without odd critical points [KSvS]. Partial results in this direction are proved in [S3,LvS2].

Quasisymmetric rigidity is a crucial ingredient in proving density of hyperbolicity, see [Ly2, GS2, GS3] for quadratic polynomials, for real polynomials without odd critical points $[\mathrm{KSvS}]$ and $[\mathrm{AKLS}]$ for unicritical polynomials. Density of hyperbolicity in the space of $C^{2}$ maps of the interval was proved in [S3]. 
Quasisymmetric rigidity can also be proved for a large class of real transcendental maps, see [RvS1] and [RvS2]. Another motivation is to extend results about monotonicity of entropy for real polynomials with only real critical points, see [BvS], to real polynomials with non-real critical points. This is work in progress by the 2 nd author joint with Cheraghi.

1.6.3. Renormalization results. In the 1970s, Feigenbaum and Coullet-Tresser [Fe, TC] observed surprising universal scaling laws in one-dimensional dynamics. They noticed that, in the family

$$
f_{\lambda}: x \mapsto x^{2}+\lambda,
$$

the sequence of points, $\lambda_{n}$, as $\lambda$ decreases from $1 / 4$, where the mapping $f_{\lambda_{n}}$ passes through a period doubling bifurcation has the following property: the ratio

$$
\frac{\lambda_{n-1}-\lambda_{n}}{\lambda_{n}-\lambda_{n+1}}
$$

converges, and even more, the limit is independent of the family of unimodal maps, provided that family of maps is chosen so that each map in the family has a nondegenerate critical point. They also observed similar universality properties of $\omega(0)$. In the period doubling case, there is a sequence of periodic intervals $J_{n}$ with period $2^{n}$ under $f$, for which the ratios $\left|J_{n}\right| /\left|J_{n+1}\right|$ converge to a fixed value, which does not depend on the choice of family. To explain these observations, they introduced the period doubling renormalization operator. They conjectured that this operator has a unique fixed point and that this fixed point is hyperbolic with a one-dimensional unstable manifold. Exponential convergence of renormalization has strong implications for the rigidity of such maps. For example:

Theorem 1.8. Assume that $f, g$ are real analytic infinitely renormalizable maps with bounded geometry. Then any topological conjugacy between $f$ and $g$ is differentiable at the critical point.

The original renormalization conjecture has been extended to cover all unimodal infinitely renormalizable combinatorial types. Roughly, the generalized version of the conjecture states that, there is a renormalization operator, $\mathcal{R}$, that acts on an appropriate space of functions, and has an invariant set, $\mathbb{K}$, called the full renormalization horseshoe. Furthermore, at each point $f \in \mathbb{K}, \mathcal{R}$ has a one-dimensional unstable manifold, and the stable manifold of $f$ corresponds to its topological conjugacy class. This conjecture was settled for quadratic maps by Lyubich, [Ly3,Ly5].

Partial proofs of such results were first obtained by [CE,La] using bounds obtained with computer assistance. Sullivan, [Su], was the first to prove convergence of renormalization for real analytic infinitely renormalizable maps of bounded type. It is precisely for this reason that he derived complex bounds for such maps. A crucial ingredient in the proof was to have that on a sufficiently small scale, the bounds are independent of the map, i.e. that these bounds are beau, see p. 5. This means that there exists a compact class $\mathcal{C}$ of maps, so that after renormalizing a map $f$, possibly a large number of times, its renormalization is in $\mathcal{C}$. Subsequent renormalization results, including exponential convergence of renormalisation, were obtained by [M1,M2,Ly3, Ly5, dFdMP]. The most recent proof in [AL] of the convergence of renormalization shows that, in fact, the property of complex bounds with beau estimates is essentially the only ingredient 
that is required. For analytic interval maps with several critical points, results on renormalization have been proved by Smania, $[\mathrm{Sm} 2, \mathrm{Sm} 4, \mathrm{Sm} 5]$. It is clear from the results just cited that our results will be a key to extending results about the hyperbolicity of renormalization to more general settings.

For critical circle maps, there is a renormalization theory that is closely related to the theory for unimodal maps, see for example [Ya2, KT, dFdM1,dFdM2].

For maps that are not real-analytic, but smooth, it turns out that some renormalization results can be obtained through asymptotically holomorphic extensions, see [GdM, GMdM] for smooth critical circle maps and [CFS] for certain smooth infinitely renormalizable interval maps.

In addition to explaining the universal scaling laws in both the phase and parameter spaces observed by Feigenbaum and Coullet-Tresser, the hyperbolicity of renormalization is a vital ingredient in the proof of the celebrated theorem that in the real family $z \mapsto z^{2}+c, c \in[-2,1 / 4]$, almost every map is regular or stochastic, [Ly5]. The hyperbolicity of renormalization, together with the fact that the leaves of the lamination of the space of polynomial-like maps by the hybrid classes are analytic manifolds, imply that the set of infinitely renormalizable maps have measure zero in generic families of unimodal maps. To complete the proof of the regular or stochastic theorem a parameter exclusion argument and a geometric characterization of stochastic mappings are used to show that in the set of non-regular, non-renormalizable parameters, almost every map is stochastic, [Ly4,MN]. This result has been generalized and improved: in generic families of analytic unimodal maps almost every map is regular or Collet-Eckmann, see [AM1, AM2, ALdM, ALS, BSvS, C].

1.7. An outline of the paper and a sketch of the proof. In this paper, we construct box mappings with complex bounds around a critical point $c_{0}$ with the property that $f$ is persistently recurrent on $\omega\left(c_{0}\right)$; recall that either $c_{0}$ is even or all critical points in $\omega\left(c_{0}\right)$ are odd. When $f$ is not persistently recurrent at $c_{0}$, one can go with bounded degree from arbitrarily small scales around $c_{0}$ to a fixed large scale; therefore for such critical points the construction of complex bounds uses rather different methods, which can be found in $[\mathrm{KSvS} 2]$ and $[\mathrm{CvS}]$. For maps with a persistently recurrent critical point $c_{0}$, we define a sequence of nested intervals around $c_{0}$ called the generalized enhanced nest

$$
I_{0} \supset I_{1} \supset I_{2} \supset \cdots \text {. }
$$

In the non-renormalizable setting, this generalized enhanced is identical to the enhanced nest in [KSvS]; however, we extend the construction so that it also covers infinitely renormalizable maps. This nest allows us to construct quasi-box mappings, see Sect. 1.7.2, in the infinitely renormalizable and non-renormalizable cases simultaneously. This is the crucial step in the construction of complex box mappings.

The enhanced nest possesses key features that play important roles throughout the proof. In the non-renormalizable case, each interval $I_{n+1}$ in the enhanced nest is a pullback of $I_{n}$ with bounded degree depending only on the vector $\underline{b}$. The combinatorics of the enhanced nest are very well controlled-if a chain starts in a deep level of the enhanced nest, and returns to it, we exploit the fact that the chain had to visit each higher level several times prior to returning. Finally, the enhanced nest provides us with dynamically defined space, free (disjoint) from $\omega\left(c_{0}\right)$, even in the (infinitely) renormalizable case. It is worth noticing that the enhanced nest is never a subsequence of the principal nest and that while the combinatorics of the enhanced nest are far more complicated than those 
of the principal nest, the principal nest does not provide the same geometric control as the enhanced nest.

1.7.1. Sections 3-8: developing the required tools. We use the same strategy to prove complex bounds as was used in [KSvS], but we extend it to allow for odd critical points and for infinitely renormalizable maps. For this reason we follow the notation from $[\mathrm{KSvS}]$, and refer as much as possible to results and proofs from that paper. We have attempted to indicate to which past results our statements correspond, even though the proofs and some of the statements require modifications. It turned out that to overcome the additional difficulties for dealing with this generality required new ideas. In particular, many of the results or proofs in Sects. 6 and 8 have no analogues in [KSvS].

Our aim is to construct a complex box mapping with complex bounds that extends $R_{I_{n}}$ for any $n$ sufficiently large. Remember that we always restrict return maps to the components that intersect $\omega\left(c_{0}\right)$. Our main goal is the construction of a quasi-box mapping. A quasi-box mapping is similar to a box mapping, except that the components of its domain need not be compactly contained in its range and we do not require the components of its domain to be pairwise disjoint (see Definition 1.2). This construction occupies most of the paper, Sections 3 to 9. In the final section, Sect. 10, we make use of quasi-box mappings to build box mappings and we show complex bounds.

To construct quasi-box mappings we use Poincaré disks based on intervals from the generalizad enhanced nest. See p. 17, for the definition of the Poincare disk $D_{\theta}(I)$, where $I$ is an interval in $\mathbb{R}$ and $\theta \in(0, \pi)$. We let $\mathbb{C}_{I}$ denote the slit complex plane $\mathbb{C}_{I}=\mathbb{C} \backslash(\mathbb{R} \backslash I)$. Let us explain the basic idea behind the construction. Fix $n$ large and let $\mathcal{L}_{x}\left(I_{n}\right)$ be the landing domain to $I_{n}$ containing a point $x \in I_{n} \cap \omega\left(c_{0}\right)$. Consider the disjoint chain $\left\{G_{j}\right\}_{j=0}^{s}$ given by $G_{s}=I_{n}$ and $G_{0}=\mathcal{L}_{x}\left(I_{n}\right)$ and let

$$
U_{s}=D_{\theta}\left(I_{n-N}\right) \cap \mathbb{C}_{I_{n}} \text { and } U_{j}=\operatorname{Comp}_{G_{j}} f^{-1}\left(U_{j+1}\right),
$$

for $0 \leq j<s$ and some fixed $3<N<n$. Assume there exists a constant $\mu \in(0,1)$ so that

$$
U_{0} \subset D_{\mu \theta}(K) \subset U_{s},
$$

where $K$ is an interval well-inside $I_{n-N}$ with $\mathcal{L}_{x}\left(I_{n}\right) \subset K$. Observe that the map $f^{s}: U_{0} \rightarrow U_{s}$ is a (qr) branched covering map. Hence, we can construct a quasi-box mapping $F: \mathcal{U} \rightarrow \mathcal{V}$, by considering $\mathcal{U}$ equal to the union of all sets $U_{0}$ constructed as above, for points $x \in I_{n} \cap \omega\left(c_{0}\right)$, and range $\mathcal{V}=D_{\theta}\left(I_{n-N}\right)$. The key part of the proof to show the existence of a beau bound on the constant $\mu$, depending only on the number of critical points of $f$ and their orders, and a universal constant $N$ so that the above construction holds for all intervals $I_{n}$ with $n$ sufficiently large. To find such constants we first study the geometric properties of the generalized enhanced nest, and then we study pullbacks of Poincaré domains in various circumstances.

Let us now survey what is done in each section.

In Sect. 3, we prove the necessary "real bounds". Before going into further detail, we refer the reader to the beginning of Sect. 3 for the definitions of $\delta$-nice and $\delta$-free. It is worth remarking that while some of the results in this section are very close to those in $[\mathrm{KSvS}]$, the proofs usually differ in significant ways. For instance, when all critical 




Fig. 1. The Poincaré disk $D_{\theta}(I)$ of angle $\theta$ based on an interval $I$

points of $f$ are even as in $[\mathrm{KSvS}]$, all intervals $I_{n}$ are $\delta$-free. However, in our setting, this need not be the case. Very roughly, using the terminology from Sect. 1.1.2, we show the existence of a universal constant $\delta>0$ such that: if $I_{n}$ is a non-terminating level of the generalized enhanced nest, then

- $\quad I_{n}$ is $\delta$-nice, and

- $\quad\left((1+2 \delta) I_{n+1} \backslash I_{n+1}\right) \cap \omega\left(c_{0}\right)=\emptyset$.

Bounded scaling factors between sufficiently many nearby levels in the enhanced nest has important consequences for the geometry of $\omega\left(c_{0}\right)$. For example, we show that if there is a small return domain to $I_{n}$, then $I_{n+1}$ is small compared to $I_{n}$, and if $I_{n}$ and $I_{n+1}$ are non-terminating, then $I_{n}$ is $\delta$-free for some $\delta>0$ depending on $\left|I_{n}\right| /\left|I_{n+1}\right|$. Furthermore, we are able to use the generalized enhanced nest and certain bounded geometry conditions to control the geometry of the post-critical set for infinitely renormalizable maps, see Proposition 3.20. Control of the post-critical set is vital throughout this paper since it makes it possible to control the shape of pullbacks of Poincare disks in the complex plane, which are the basic pieces we will use to construct quasi-box mappings.

In Sect. 4, we present some necessary facts about Poincaré disks and show how to pull them back by first return maps. When we pull back one of these domains (i.e. we take its preimage) by a unicritical branched covering, knowing that the critical values of the map are not close to the boundary of the disk, allows us to control the shape of this pullback, see Lemmas 4.2 and 4.3. Of greatest importance to us is that we control the loss of angle. The pullback of a Poincaré disk with angle $\theta$ is contained in a Poincaré disk of angle $\theta^{\prime} \leq \theta$ and we bound $\theta^{\prime}$ from below (in terms of $\theta$ ), which gives us some control on the geometry of the pullbacks (Figs. 1, 2).

Asymptotically holomorphic extensions were used by Lyubich to prove complex bounds and quasisymmetric rigidity for the quadratic Fibonacci map [Ly1]. For an application to infinitely renormalizable mappings with bounded combinatorics see [Su]. In [GSS2] the theory of asymptotically holomorphic extensions was developed further, and we use their results in our proof. In Sect. 5, we recall these results, and use them 

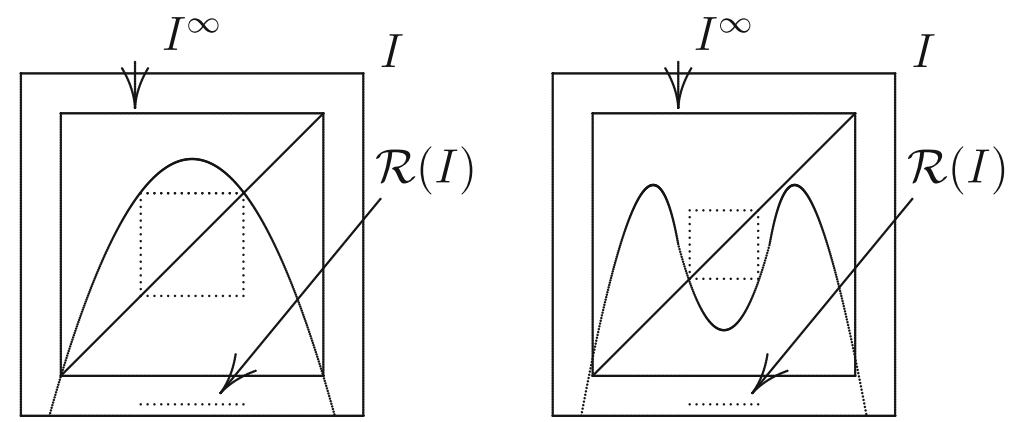

Fig. 2. The figure shows two cases where $I$ is terminating together with the interval $\mathcal{R}(I)$ marked in dots

to develop tools for dealing with $C^{3}$ maps $f: M \rightarrow M$. These maps yield quasiregular extensions of $C^{3}$ interval maps to a neighbourhood in the complex plane, which are asymptotically holomorphic. The key property of these extensions is that, like analytic maps, they satisfy an "Almost Schwarz Inclusion Principle:" the loss of angle when pulling back a Poincaré disk by a diffeomorphism is small, see Lemma 5.5. We also show in this section that the results in Sect. 4 go through in the smooth setting. The reader who is only interested in the main theorem in the real analytic setting can skip this section.

In Sect. 6, we show how to pull back Poincaré disks in a few different situations: along monotone branches, by maps of bounded degree, and through long cascades of central returns. All these arguments are complicated by the fact that our map is not a polynomial; therefore we will lose angle even when we pull back by a diffeomorphism.

In Propositions 6.1 and 6.3, the problem that we face is pulling back a slit Poincare disk $D_{\theta}\left(\hat{G}_{s}\right) \cap \mathbb{C}_{G_{s}}$ along a chain $\left\{G_{j}\right\}_{j=0}^{s}$ under which the chain $\left\{\hat{G}_{j}\right\}_{j=0}^{s}$ could have any degree. To deal with this issue, we make use of dynamically defined free space and the existence of fundamental domains. Of particular importance is Proposition 6.1 (which has no analogue in $[\mathrm{KSvS}]$ ). This proposition allows us to pull back along a single monotone branch for as long as we like, using fundamental domains of definite size to control the loss of angle.

Proposition 6.3 allows us to pull back along long cascades of central returns. It is similar to [KSvS, Proposition 11.1]; however, it also covers the case of terminating intervals. Its proof, compared to its analogue in [KSvS], is complicated by the fact each time we pullback along a monotone branch we lose some angle, so we have to bound the number of times we switch between monotone branches. This argument is new and non-trivial; ideas of this proof are also used in Sect. 7.

In Sect. 7 we use the notion of combinatorial depth of a chain from [KSvS]. For a given critical point the combinatorial depth measures how close a chain comes to the critical point $c$ in terms of the number central cascades around $c$ that the chain enters. Proposition 7.1 bounds the loss of angle for the pullback of the Poincare disk along a chain in terms of its combinatorial depth.

In Sect. 8, we show how to control the loss of angle as we pull back from one level of the enhanced nest to the next, under two different circumstances: bounded scaling factors and big scaling factors. The first case, which occupies the majority of the section, is roughly explained as follows. There exist $s_{i}<s_{i-1}, \lambda \in(0,1)$ and $\delta>0$, so that 


$$
\operatorname{Comp}_{G_{s_{i}}} f^{-\left(s_{i-1}-s_{i}\right)}\left(D_{\theta}\left(I_{n-i-1}\right) \cap \mathbb{C}_{G_{s_{i-1}}}\right) \subset D_{\lambda \theta}\left((1+2 \delta)^{-1} I_{i}\right) .
$$

This argument and the strategy of the proof are subtle, in particular due the presence of terminating intervals. We must control the combinatorial depth of (certain segments of) the chain $\left\{G_{j}\right\}_{j=0}^{s_{i}}$, which can be guaranteed only when the scaling factor between $I_{n-i-1}$ and $I_{n-i+1}$ is bounded. In this part of the proof, the difference between the non-renormalizable and the renormalizable cases is pronounced. Roughly, in the nonrenormalizable case we are able to decompose the map $f^{s_{i-1}-s_{i}} \mid D_{\theta}\left(I_{n-i-1}\right) \cap \mathbb{C}_{G_{s_{i-1}}}$ into a bounded sequence of that maps that we can control, and use the results of the previous sections to control the loss of angle at each stage. This is impossible to do when there are terminating intervals in the generalized enhanced nest; if $I_{n-i-1}$ is terminating, $I_{n-i}$ is not a pullback of $I_{n-i-1}$. In this case, we pull back from a terminating interval in the enhanced nest to the next level in two stages: first we pull back from the terminating interval to the largest periodic interval contained in it, and then we pull back from the periodic interval to the next level of the generalized enhanced nest. This strategy is described in Diagrams 13 and 14.

When there are big scaling factors between levels of the enhanced nest, it is enough to make use of the dynamically defined external free space to control the loss of angle. Furthermore, big scaling factors allow us to construct complex box mappings without the aid of quasi-box mappings.

1.7.2. Section 9: construction of quasi-box mappings. In this section we use an inductive argument to construct a quasi-box mapping that extends the return mapping to any interval $I_{n}$ of the enhanced nest, provided $n$ is sufficiently large. The notion of a quasibox mapping was introduced in [LvS1, Remark 12.4], used explicitly in [LvS2], and is defined as follows:

Definition 1.2. Let $U_{i}$ and $V_{j}$ be open Jordan disks in $\mathbb{C}, 0 \leq j<b, i \in \mathcal{I}$ where $\mathcal{I}$ is at most countable. Set $\mathcal{U}=\cup U_{i}$ and $\mathcal{V}=\cup V_{j}$. A mapping $F: \mathcal{U} \rightarrow \mathcal{V}$ is a holomorphic quasi-box mapping if for each $i$, there exists $j$ and a holomorphic branched covering $F_{i}: U_{i} \rightarrow V_{j}$, and the following hold:

- $V_{0}, \ldots, V_{b-1}$ are pairwise disjoint Jordan disks;

- every connected component $V_{j}$ of $\mathcal{V}$ is either a connected component of $\mathcal{U}$ or the intersection of $V_{j}$ with $\mathcal{U}$ is a union of sets $U_{i}$ where each of these is contained in $V_{j}$, not necessarily compactly;

- if $U_{i} \subset V_{j}$, then $\bar{U}_{i} \backslash V_{j} \subset \mathbb{R}$.

Notice that we do not assume the components of $\mathcal{U}$ are disjoint, so $F: \mathcal{U} \rightarrow \mathcal{V}$ may be multi-valued, but $F \mid U_{i}=F_{i}$ is well-defined as a single valued function on each $U_{i}$. If we only require that $F_{i}$ be quasi-regular on each $U_{i}$ then we say that $F$ is a qr quasi-box mapping, see Sect. 5.3. When it will not cause confusion, we will refer to these mappings as quasi-box mappings.

We define the filled Julia set of a quasi-box mapping $F: \mathcal{U} \rightarrow \mathcal{V}$ as the set

$$
K(F)=\left\{z \in \mathcal{U}: F^{k}(z) \in \mathcal{U} \text { for all } k=0,1,2, \ldots\right\} .
$$

To construct a qr quasi-box mapping we only need to control the loss of angle when we pull back a Poincaré disks under a certain chain. The angle control that we have is the same for both the asymptotically holomorphic extensions we use as it is for analytic 
maps, so the proofs in the smooth and analytic cases are essentially the same. It is worth remarking that we do not control the dilatation of a qr quasi-box mapping; the presence of long central cascades seems to make this impossible using our methods.

We start the construction of quasi-box mappings with a Poincare disk, based on a slit domain

$$
D_{\theta}\left(I_{n-M}\right) \cap \mathbb{C}_{I_{n}} \text { where } \mathbb{C}_{I_{n}}=\mathbb{C} \backslash\left(\mathbb{R} \backslash I_{n}\right)
$$

and pull it back from one level of the enhanced nest to the next using the arguments in Sect. 8 .

If the scaling factor between the level $I_{n-i}$ and $I_{n-i+1}$ is ever big enough, we can easily construct a box mapping for the return map to a deeper puzzle piece, which gives us a quasi-box mapping for the return map to deeper levels. So in what follows we assume the scaling factors between the level $I_{n-i}$ and $I_{n-i+1}$ are bounded.

The combinatorics of the enhanced nest make it possible to select times to pull back to in such way that once we have pulled back to level $I_{n-i}$, the forward orbit of the chain only visits $I_{n-i}$ a couple of times. This bound is used in following way: if $I_{n-i}$ is comparable to $I_{n}$, then by the real bounds, the components of the domain of the return map to $I_{n-i}$ are comparable to $I_{n-i}$, so the derivative of the return mapping to $I_{n-i}$ is bounded. Since the number of returns of the chain $G_{S_{n-i}}, \ldots, G_{S}$ to $I_{n-i}$ is bounded, we have that $G_{s_{n-i}}$ is comparable to $I_{n}$, and hence to $I_{n-i}$ too. In the presence of terminating intervals, the proof of the existence of suitable times to pull back to, at each level, involves a combinatorial analysis of the return maps. Let us explain the pull back argument in more detail. Let $x \in \omega(c) \cap I_{n}$ and consider the chain $\left\{G_{j}\right\}_{j=0}^{S}$ where $G_{S}=I_{n}$ and $G_{0}=\mathcal{L}_{x}\left(I_{n}\right)$. From Sect. 8, we know there exist $s_{n-M+1}<s, \lambda \in(0,1)$ and $\delta>0$ such that

$$
\operatorname{Comp}_{G_{s_{n-M+1}}} f^{-\left(s-s_{m-M+1}\right)}\left(D_{\pi / 2}\left(I_{n-M}\right) \cap \mathbb{C}_{I_{n}}\right) \subset D_{\lambda \pi / 2}\left((1+2 \delta)^{-1} I_{n-M+1}\right) .
$$

In other words, as we pull back a point from one level of the enhanced nest to the next, we know how much angle we lose. If we do not lose any angle; that is, for the set of points in

$$
\operatorname{Comp}_{G_{s_{n-M+1}}} f^{-\left(s-s_{m-M+1}\right)}\left(D_{\pi / 2}\left(I_{n-M}\right) \cap \mathbb{C}_{I_{n}}\right) \cap D_{\pi / 2}\left(I_{n-M+1}\right),
$$

we repeat the argument and control the loss of angle up to a time $s_{n-M+2}<s_{n-M+1}$ with a Poincaré disk based on $I_{n-M+2}$. This argument can be carried on inductively, because of the combinatorics of the enhanced nest. If we lose angle when we pull back; that is, for the set of points in

$$
\operatorname{Comp}_{G_{s_{n-M+1}}} f^{-\left(s-s_{m-M+1}\right)}\left(D_{\pi / 2}\left(I_{n-M}\right) \backslash D_{\pi / 2}\left(I_{n-M+1}\right)\right),
$$

then this is a "jumping time" associated to this point (see [LY]), and also Fig. 11 on p. 61. In this case, since $G_{S_{n-M+1}}$ is comparable to $I_{n-M+1}$, as assumed above, there exists $\lambda^{\prime} \in(0,1)$ so that

$$
\operatorname{Comp}_{G_{s_{n-M+1}}} f^{-\left(s-s_{m-M+1}\right)}\left(D_{\pi / 2}\left(I_{n-M}\right) \cap \mathbb{C}_{I_{n}}\right) \backslash D_{\pi / 2}\left(I_{n-M+1}\right) \subset D_{\lambda^{\prime} \pi / 2}\left(G_{s_{n-M+1}}\right),
$$

and we are able to capture this set by a Poincaré disk based on $G_{S_{n-M+1}}$ without losing too much angle. Since the chain $\left\{G_{i}\right\}_{i=0}^{s-1}$ is disjoint, it is not hard to control the loss of angle as we pull back all the way to the start. So there are two cases, given a point 
$z \in D_{\pi / 2}\left(I_{n-M}\right) \cap \mathbb{C}_{I_{n}}$ either the point $f^{-\left(s-s_{n-i}\right)}(z) \subset D_{\pi / 2}\left(I_{n-i}\right)$ for all $i$, or there exists some $i$, for which $s_{n-i}$ gives a jumping time. In either case, choosing $M$ properly, we show $f^{-s}(z) \subset D_{\pi / 2}\left(I_{n-1}\right)$. Hence, the return map to $I_{n}$ extends to a quasi-box mapping. This is Theorem 9.3. It is important to remark that, throughout this section, the presence of periodic intervals significantly complicates the arguments.

1.7.3. Section 10: construction of complex box mappings with complex bounds. In this final section, we use quasi-box mappings to construct box mappings. In the nonrenormalizable case, we employ a method that was first used in [LvS2]: we first construct 'by hand' a smooth box mapping with the desired topological properties: the domain being compactly contained in the range and the components of the domain being disjoint, and then intersect it with the quasi-box mapping to obtain a complex box mapping. In the renormalizable case we make use of a result of [LY]. We then prove that complex bounds hold for these complex box mappings. The work in this section is done primarily to deal with the smooth, as opposed to the analytic, case. The complex bounds for at most finitely renormalizable maps follow immediately from the "Upper and Lower Bounds," see $[\mathrm{KSvS}]$ :

Proposition 1.9 (Upper bounds). There exists a constant $\eta>0$ such that for all $n$ sufficiently large the following hold.

- $\operatorname{diam}\left(\boldsymbol{I}_{n}\right) \leq \eta\left|I_{n}\right|$, and;

- there exists a topological disk $\Omega \supset \boldsymbol{I}_{n}$ such that $\left(\Omega \backslash \boldsymbol{I}_{n}\right) \cap \omega\left(c_{0}\right)=\emptyset$ and

$$
\bmod \left(\Omega \backslash \boldsymbol{I}_{n}\right)>1 / \eta
$$

Proposition 1.10 (Lower bounds). There exist beau constants $\eta>0$ and $\varepsilon>0$ such that for all $n>0$ with $\left|I_{n}\right|<\varepsilon$,

$$
B\left(c_{0}, \eta\left|I_{n}\right|\right) \subset \boldsymbol{I}_{n}
$$

For infinitely renormalizable maps, the key estimate is to show that if $F^{\prime}: U^{\prime} \rightarrow V^{\prime}$ is the quasi-box mapping map constructed in Sect. 9 that extends the return map to a periodic interval $J$ of sufficiently high period, then $\bmod \left(V^{\prime} \backslash K\left(F^{\prime}\right)\right)$ is bounded away from zero. From this we obtain a polynomial-like map that extends the return map to $J$ with complex bounds from Lemma 10.17.

1.8. Relation to complex bounds for non-renormalizable polynomials: [KvS]. Up to Sect. 10.2, this paper is concerned with the construction of a complex box mapping associated to a real map of the interval. Before this is accomplished, the methods of [KvS] do not seem to apply. However, once we have constructed a complex box mapping, in the analytic case, it is possible to replace the arguments of this paper with the arguments of $[\mathrm{KvS}]$ to establish complex bounds.

In fact, it is possible to generalize [KvS, Lemma 9.1 (Small Distortion of Thin Annuli)] to the case when $F: \mathcal{U} \rightarrow \mathcal{V}$ is quasiregular, and obtain a similar statement. This gives an alternative approach to establishing complex bounds when $f \in \mathcal{A}_{\underline{b}}^{3}$ is non-renormalizable. However, with the preparation that we have already done in this paper, the approach of $[\mathrm{KSvS}]$ is more natural, and that is the route we take to prove complex bounds. 
1.9. The complications of having smooth maps, with critical points of odd order which are possibly infinitely renormalizable. Let us highlight some of the main complications in our proof, compared to the proof of complex bounds in [KSvS] in the nonrenormalizable case and to $[\mathrm{S} 3]$ in the infinitely renormalizable one.

1.9.1. Diffeomorphic pullbacks. In both the analytic and the $C^{3}$ settings, we have a loss of angle along diffeomorphic pullbacks of Poincaré disks (see Lemmas 5.5 and 4.1). To bound this loss, we need to ensure that certain chains of intervals are disjoint or have that the sum of the lengths of their elements are bounded. This is not a problem in the polynomial case where there is no loss of angle under diffeomorphic pullbacks.

1.9.2. Odd critical points. If $\omega\left(c_{0}\right)$ contains only critical points of odd order, then the real bounds require a more elaborate treatment than if at least one of the critical points is even. This is due to the lack of symmetry around critical points of odd order, i.e. an odd critical point need not be close to the centre of a first return domain. As a result, the statement and the proof of the real bounds become more subtle. Indeed, Theorem 3.1 covers more cases than the corresponding statement in [KSvS], namely Proposition 8.1.

When there are any odd critical points, we need to deal with saddle-cascades occurring in monotone branches. We do this in Proposition 6.1. This is used in Proposition 6.3, which is a key step in the proof, and it is a generalization of [KSvS, Lemma 11.1]. To prove this proposition, we need to control the number of times we switch from one monotone branch to another since each time we switch we lose angle. To make the argument work requires a subtle change to the proof of Lemma 11.1 in [KSvS].

1.9.3. Infinitely renormalizable maps. If the map is infinitely renormalizable, then the argument used to prove complex bounds in $[\mathrm{KSvS}]$ no longer applies. For this reason we define the generalized enhanced nest, see Sect. 2.5. One of the advantages of working with this nest is that it has better geometric properties than the principle nest, which was used in [S3]. Indeed, we use the control on the geometry of the post-critical set, which we obtain under some straightforward bounded geometry conditions, to pull back from one level of the generalized enhanced nest to the next. This is done in Sect. 8.1, see also diagrams in Figs. 13 and 14, see p. 68.

1.10. Notation and terminology. Unless otherwise stated, we adopt the convention that $C>0$ is a large constant, and $\varepsilon>0$ will be a small constant.

1.10.1. Scaled sets, Poincaré domains and components. We let $\mathbb{R}$ be the real line. We will always use $I$ to denote an interval in $\mathbb{R}$. If $I$ is a bounded interval, then write $I=(a+x, a-x)$ and for $\gamma>0$ define $\gamma I:=(a+\gamma x, a-\gamma x)$. We let $\mathbb{C}$ denote the complex plane. We let $\mathbb{C}_{I}=\mathbb{C} \backslash(\mathbb{R} \backslash I)$. We let $\mathbb{H}$ be the upper-half plane and $\mathbb{H}^{-}$ be the lower-half plane. If $\theta \in(0, \pi)$, we denote by $D_{\theta}^{+}(I)$ (respectively $\left.D_{\theta}^{-}(I)\right)$ the region in $\mathbb{H}$ (respectively $\mathbb{H}^{-}$) bounded by $I$ together with the circle arc subtending $I$ that meets the real axis with external angle $\theta$ at each boundary point of $I$. We let $D_{\theta}(I)=D_{\theta}^{+}(I) \cup D_{\theta}^{-}(I) \cup I$. This set corresponds to the set of points with a fixed distance to $I$ in the Poincaré metric in $\mathbb{C}_{I}$. Given a set $K \subset \mathbb{C}$ we let $\operatorname{Comp}_{x}(K)$ denote the connected component of $K$ containing $x$. 
1.10.2. Definition of the class of functions. We let $\mathcal{A}_{b}^{k}$ be the set of $C^{k}$ maps of the interval $[0,1]$, (i.e. are $C^{k}$ on some small neighbourhood of $[0,1]$ ), with critical points $\left(c_{1}, \ldots, c_{b}\right)$ with integer orders $\underline{b}=\left(\ell_{1}, \ldots, \ell_{b}\right)$, where $b=|\underline{b}|$ is the number of critical points, such that at each point $c_{i} \in[0,1]$ we can express $f$ locally as $f(x)=$ $\left[\phi_{i}\left(x-c_{i}\right)\right]^{\ell_{i}}+f\left(c_{i}\right)$ where $\phi_{i}$ is a local $C^{k}$ diffeomorphism, $\phi_{i}(0)=0$ and $\ell_{i}>0$. If $\ell_{i}$ is even (odd) we say that the corresponding critical point $c_{i}$ has even (odd) order. We will call a critical point of even order a turning point. We let $\mathcal{A}_{b}$ denote the set of such maps that are analytic. As usual, we will say that a map is univalent if it is holomorphic and injective.

1.10.3. First return maps, pullbacks, periodic intervals and nice intervals. As usual, we let $\omega(x)$ denote the omega-limit set of a point $x$.

Given a set $K \subset \mathbb{C}$ we will only consider branches of $R_{K}$ (the first return map to $K$, see p.3) that intersect $\omega\left(c_{0}\right)$ for a fixed critical point $c_{0}$. Where $c_{0}$ will be chosen later.

Let $f: M \rightarrow M$ be a continuous interval map. An interval $J$ is called a pullback of an interval $I$ if it is a component of $f^{-s}(I)$ for some $s \in \mathbb{N}$. If $J$ is a pullback of $I$ by $f^{-s}$, we associate to this pullback a chain of intervals $\left\{J_{i}\right\}_{i=0}^{s}$ with $J_{s}=I$ and $J_{i}=\operatorname{Comp}_{f^{i}(J)}\left(f^{-1}\left(J_{i+1}\right)\right)$ for $i$ satisfying $0 \leq i<s$. We say that the order of the chain $\left\{J_{i}\right\}_{i=0}^{S}$ is $N$ if precisely $N$ of the intervals $J_{0}, \ldots, J_{s-1}$ contain a critical point.

We say that an open interval $J$ is nice if $f^{n}(x) \notin \operatorname{int}(J)$ for each $x \in \partial J$ and $n>0$. This implies that for any $k>n \geq 0$, if a component $J_{k}$ of $f^{-k}(J)$ intersects a component $J_{n}$ of $f^{-n}(J)$ then $J_{k}$ is contained in $J_{n}$; so any two pullbacks of a nice interval are either nested or disjoint. In particular, if the first return time of $x$ to $J$ is equal to $s$ and we consider the chain $\left\{J_{i}\right\}_{i=0}^{s}$ with $J_{s}=I$ and $J_{i}=\operatorname{Comp}_{f^{i}(x)}\left(f^{-1}\left(J_{i+1}\right)\right)$ we get the following: $J_{0}=\mathcal{L}_{x}(J)$, the intervals $J_{0}, \ldots J_{s-1}$ are pairwise disjoint and the order of the chain $\left\{J_{i}\right\}_{i=0}^{s}$ is bounded by the number of critical points of $f$.

We will say that two intervals $I$ and $J$ have nested or disjoint pullbacks if for any $m, n \in \mathbb{N}$, any component $I_{1}$ of $f^{-m}(I)$ and any component $J_{1}$ of $f^{-n}(J)$ either $J_{1}$ and $I_{1}$ are nested or $J_{1}$ and $I_{1}$ are disjoint.

Give two nice intervals $J \subset I$, they are called a nice pair if all iterates of $\partial J$ remain outside the interior of $I$. Under these circumstances, if $J_{1}, J_{2}$ are pullbacks of $J$ and $I_{1}, I_{2}$ are pullbacks of $I$ with $J_{i} \subset I_{i}$ for $i=1,2$ and $I_{2} \subset I_{1}$, then either

$$
J_{2} \subset I_{2} \subset J_{1} \subset I_{1} \text { or } J_{2} \subset I_{2} \subset I_{1} \backslash J_{1} \text {. }
$$

1.10.4. Notation table. At the end of the paper, there is a table of some of the notation and terminology we use throughout.

\section{The Generalized Enhanced Nest}

2.1. Real puzzle pieces. Let us assume that $f: M \rightarrow M$ is a $C^{3}$ map of the interval with $b<\infty$ critical points and let $c$ be a recurrent, non-periodic critical point. We say that a set $Z$ is admissible if it is a finite forward invariant set, disjoint from the postcritical set of $f$ such that every point of $Z$ is a preimage of a repelling periodic point under an iterate of $f$.

Given a $Z$ admissible set, we will say that $I$ is a (real) puzzle piece of depth $n$ (with respect to $Z$ ) if it is a component of $f^{-n}(Y)$, where $Y$ is a component of $M \backslash f^{-1}(Z)$. We observe that puzzle pieces are nice intervals, so any two puzzle pieces are either nested or disjoint. 
2.2. Combinatorics of puzzle pieces. While the objects in this subsection are defined for real puzzle pieces, the definitions in this subsection hold whether a puzzle piece is real or complex.

We say that a puzzle piece is $\omega(c)$-critical if it contains a critical point in $\omega(c)$. Let $P$ be an $\omega(c)$-critical puzzle piece containing the critical point $c^{\prime} \in \omega(c)$. An $\omega(c)$ critical puzzle piece $Q$ is a called a child of $P$ if it is a unicritical pullback of $P$; that is, there exists a positive integer $n$ such that $Q$ is a component of $f^{-n}(P)$ containing a critical point in $\omega(c)$, and there exists a puzzle piece $Q^{\prime} \supset f(Q)$ such that the map $f^{n-1}: Q^{\prime} \rightarrow P$ is a diffeomorphism. A successor of $P$ is a puzzle piece of the form $\hat{\mathcal{L}}_{c^{\prime}}(Q)$, where $Q$ is a child of $\hat{\mathcal{L}}_{c^{\prime \prime}}(P)$ for some critical point $c^{\prime \prime} \in \omega(c)$. By construction, a successor of $P$ is a pullback of $P$ of order bounded by $2 b-1$.

Let $I$ be a puzzle piece containing a recurrent point $x$. We define the principal nest around $x$ as follows. We set $I^{0}=I$ and inductively define $I^{n+1}=\mathcal{L}_{x}\left(I^{n}\right)$. Given a puzzle piece $I^{n}$ the puzzle piece $I^{n+1}$ will be called central, while any other return domain to $I^{n}$ will be called non-central.

Let $c$ be a recurrent, non-periodic, critical point and consider the principal nest $I^{0} \supset$ $I^{1} \supset I^{2} \supset \cdots$ about $c$. We define $\hat{m} \in \mathbb{N} \cup\{\infty\}$ to be the smallest number, if it exists, such that a there is a critical point $c^{\prime}$ of $R_{I^{0}} \mid I^{1}$ with $\left(R_{I^{0}} \mid I^{1}\right)\left(c^{\prime}\right) \notin I^{\hat{m}}$. If no such integer exists, we set $\hat{m}=\infty$. If $\hat{m}<\infty$ we say that $I$ is non-terminating and otherwise we say that $I$ is terminating. In the terminating case, we let $I^{\infty}=\cap_{i \geq 0} I^{i}$.

2.3. Persistent recurrence. A map $f$ is called persistently recurrent on $\omega(c)$ if $c$ is recurrent, non-periodic and each $\omega(c)$-critical puzzle piece has only finitely many children. Under these circumstances, we will also say that $f$ is persistently recurrent at $c$ and that $c$ is a persistently recurrent critical point. If $f$ is persistently recurrent on $\omega(c)$, then $\omega(c)$ is minimal, but the converse of that statement is false. We observe the following, if $f$ is persistently recurrent on $\omega(c)$ each $\omega(c)$-critical puzzle piece $P$ has a smallest successor, which we denote by $\Gamma(P)$; and if $Q$ is an entry domain to $P$ intersecting $\omega(c)$, then $\hat{\mathcal{L}}_{c}(Q)$ is a successor of $P$, and thus $P \supset \hat{\mathcal{L}}_{c}(Q) \supset \Gamma(P)$.

From $[\mathrm{KSvS}]$, pp. 771-772, we know the following:

Lemma 2.1. If $f$ is persistently recurrent on $\omega(c)$, then for any $\varepsilon>0$ there exists an admissible set $Z_{\varepsilon}$ such that any real puzzle piece of depth zero that intersects $\omega(c)$ has length less than $\varepsilon$.

As a direct consequence of the No Wandering Intervals Theorem, see [vSV, p. 751], we get that for any $\delta>0$ there exists $\varepsilon(\delta, f)>0$ such that the length of any pullback of a critical real puzzle piece with size less than $\varepsilon$ has size less than $\delta$. From this fact and Lemma 2.1, we can assume that any real puzzle piece that intersects $\omega(c)$ has length less than $\varepsilon_{0}$, where we can choose $\varepsilon_{0}>0$ as small as we like. We will use this observation without further comment.

Remark. Suppose that $c \in \operatorname{Crit}(f)$ and that $f$ is persistently recurrent on $\omega(c)$. Then for any sufficiently small nice interval $I \ni c$ the following holds.

- The interval $I$ contains no other point from $\operatorname{Crit}(f)$.

- If $c^{\prime} \in \operatorname{Crit}(f)$ is any critical point such that there exists a chain $\left\{G_{i}\right\}_{i=0}^{S}$, with $G_{s}=I$ and $G_{0}=\mathcal{L}_{x}(I)$ for $x \in \omega(c) \cap I$, and $c^{\prime} \in G_{j}$ for some $0 \leq j<s$, then $c^{\prime} \in \omega(c)$. 
Assume $f$ is persistently recurrent on $\omega(c)$. By Lemma 2.1 and the remark above we know that (for $I$ is sufficiently small) all critical values of $\left.f^{r}\right|_{I^{1}}$ are contained in $\omega(c)$. Since $f$ is persistently recurrent, $\omega(c)$ is not a periodic orbit. These two facts, along with [dMvS, Theorem III.4.1] imply $\left.f^{r}\right|_{I^{1}}$ does not have a periodic attractor (otherwise $\omega(c)$ would be a periodic orbit). Moreover, since $\omega(c)$ is minimal, and the period of all attracting or parabolic cycles is bounded $[\mathrm{dMvS}$, Chapter IV, Theorem B], there exists a neighbourhood of $\omega(c)$ which does not intersect any immediate basin of a periodic attractor or any parabolic cycle. Thus if $I$ is sufficiently small, for any $x \in \omega(c)$, the chain $\left\{G_{j}\right\}_{j=0}^{s}$ with $G_{s}=I$ and $G_{0}=\mathcal{L}_{x}(I)$ avoids a neighbourhood of any immediate basin of a periodic attractor or any parabolic point.

It will be useful for us to select a critical point $c_{0} \in \operatorname{Crit}(f)$ about which we will focus our construction. If $\omega(c)$ contains a turning point, we take $c_{0}$ to be a turning point, otherwise choose $c_{0} \in \omega(c) \cap \operatorname{Crit}(f)$ arbitrarily. Observe that $f$ is persistently recurrent on $\omega\left(c_{0}\right)$, so $c_{0}$ is recurrent, non-periodic, $\omega\left(c_{0}\right)$ is minimal and $\omega(c)=\omega\left(c_{0}\right)$.

2.4. Terminating intervals. Suppose that $I \ni c_{0}$ is a terminating interval. Then, since $c$ is recurrent and non-periodic, $R_{I}$ has a critical point of even order, and hence $c_{0}$ is of even order too. Since $c_{0}$ is a turning point, there exists a neighbourhood $J \supset I$ of $c_{0}$ and an involution $\tau: J \rightarrow J$ so that $f=f \circ \tau$ on $J$. Let $r$ be the return time of $c_{0}$ to $I$. Since $I$ is terminating, it follows that $I^{\infty}$ is a periodic interval. More precisely, $f^{r}\left(I^{\infty}\right) \subset I^{\infty}$, and $f^{r}\left(\partial I^{\infty}\right) \subset \partial I^{\infty}$ and all of the critical points of $f^{r} \mid I^{1}$ are contained in $I^{\infty}$ along with their orbits under $f^{r}$. We let $\beta$ denote the fixed point of $f^{r}$ on the boundary of $I^{\infty}$

Since $c_{0} \in I^{\infty}$, a periodic interval, and $c_{0}$ is recurrent, $f^{r} \mid I^{\infty}$ has at least one repelling orientation reversing fixed point. Let $\alpha$ be the orientation reversing fixed of $f^{r} \mid I^{\infty}$ closest to $c_{0}$; so $(\alpha, \tau(\alpha)) \ni c_{0}$ is the smallest $\tau$-symmetric interval with one repelling fixed point on its boundary.

Given a terminating interval $I$ we define $\mathcal{R}(I)$ as

$$
\mathcal{R}(I):=(\alpha, \tau(\alpha)) .
$$

See Fig. 12 for some examples. If $\mathcal{R}(I)$ is a periodic interval, then it has period two under $R_{I}$ and we say $\mathcal{R}(I)$ is Feigenbaum.

We let $Y_{i}$ with $-a \leq i \leq a$ denote the components of $I^{\infty} \backslash f^{-r}(\alpha)$ labeled as follows: $Y_{0}=\mathcal{R}(I), Y_{-1} \neq Y_{0}$ is the other component that contains $\alpha$ in its boundary and $Y_{-a}$ and $Y_{a}$ are the components that contain $\beta$ and $\tau(\beta)$, respectively, in their boundaries (see Fig. 3). We let $\tilde{\mathcal{Y}}_{\gamma}$ denote the monotone branch of $f^{r} \mid I^{\infty}$ that contains $\gamma$, where $\gamma \in\{\alpha, \beta, \tau(\beta)\}$ (see Fig. 3).

2.5. The generalized enhanced nest. We will extend the construction of the enhanced nest of $[\mathrm{KSvS}]$ to cover the renormalizable case. We will make use of the following combinatorially defined return time.

Lemma 2.2 ([KSvS], Lemma 8.2). Let I $\ni$ c be a $\omega\left(c_{0}\right)$-critical puzzle piece. Then there exists a positive integer $v$ with $f^{v}(c) \in I$ such that the following holds. Let $U_{0}=\operatorname{Comp}_{c} f^{-v}(I)$ and $U_{j}=\operatorname{Comp}_{f^{j}(c)} f^{-(v-j)}(I)$ for $0 \leq j \leq v$. Then

(1) $\#\left\{j: U_{j} \cap \operatorname{Crit}(f) \neq \emptyset, 0 \leq j \leq v-1\right\} \leq b^{2}$, and

(2) $U_{0} \cap \omega(c) \subset \operatorname{Comp}_{c}\left(f^{-v}\left(\mathcal{L}_{f^{v}(c)}(I)\right)\right)$. 


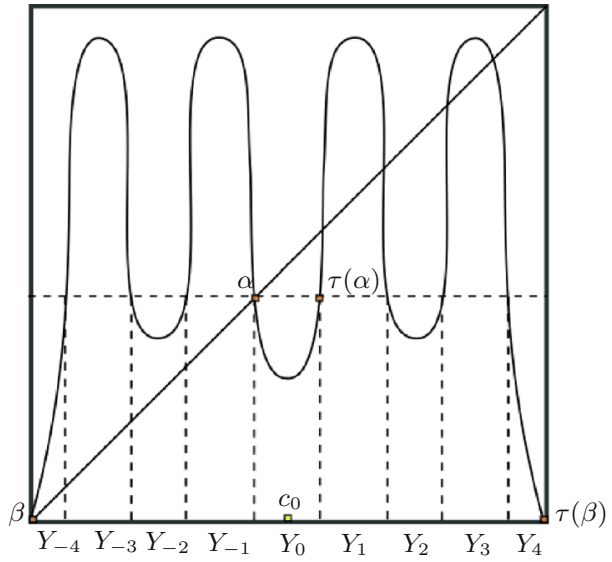

a



Fig. 3. a Partition of $I^{\infty}$. b Labelling of monotone branches

For each puzzle piece $I \ni c_{0}$ we let $v=v(I)$ be the smallest positive integer with the properties specified by Lemma 2.2. We define

$$
\begin{aligned}
& \mathcal{A}(I)=\operatorname{Comp}_{c_{0}} f^{-v}\left(\mathcal{L}_{f^{v}\left(c_{0}\right)}(I)\right), \\
& \mathcal{B}(I)=\operatorname{Comp}_{c_{0}} f^{-v}(I) .
\end{aligned}
$$

By construction $\mathcal{A}(I) \subset \mathcal{B}(I)$ and $(\mathcal{B}(I) \backslash \mathcal{A}(I)) \cap \omega\left(c_{0}\right)=\emptyset$, giving a mechanism for obtaining free space, i.e. space disjoint from $\omega\left(c_{0}\right)$, on the outside and inside of the interval $\mathcal{B}(\mathcal{A}(I))$. See Fig. 4.

Next, let $T=5 b$, where $b$ is the number of critical points in $\omega(c)$, and define

$$
\mathcal{E}(I)= \begin{cases}\Gamma^{T} \mathcal{B} \mathcal{A}(I) & \text { if } I \text { is non-terminating }, \\ \mathcal{L}_{c_{0}}(\mathcal{R}(I)) & \text { if } I \text { is terminating. }\end{cases}
$$

The generalized enhanced nest associated to a nice interval $I \ni c_{0}$ is inductively defined by

$$
I_{0}=I \quad \text { and } \quad I_{n+1}=\mathcal{E}\left(I_{n}\right) \text { for } n \geq 0 .
$$

For simplicity, we will refer to the elements from this nest as intervals from the enhanced nest. However it is important to remark this definition differs from the one

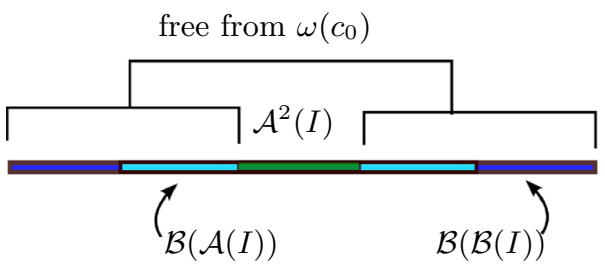

Fig. 4. The intervals shown are $\mathcal{A}^{2}(I) \subset \mathcal{B}(\mathcal{A}(I)) \subset \mathcal{B}^{2}(I)$. The pair of intervals $\mathcal{B}^{2}(I) \backslash \mathcal{A}^{2}(I)$ is disjoint from the postcritical set 
introduced in $[\mathrm{KSvS}]$ in the presence of terminating intervals. If $I_{n}$ is non-terminating, we let $p_{n}>0$ be so that $I_{n+1}$ is a component of $f^{-p_{n}}\left(I_{n}\right)$. Under these circumstances, Lemma 2.2 and the definition of successor imply that $I_{n+1}$ is a pullback of $I_{n}$ of bounded order; $\mathcal{B A}\left(I_{n}\right)$ is a pullback of $I_{n}$ with order bounded from above by $2 b^{2}$, and each successor $\Gamma^{i+1}\left(\mathcal{B} \mathcal{A}\left(I_{n}\right)\right)$ is a pullback of order at most $2 b-1$ of $\Gamma^{i}\left(\mathcal{B A}\left(I_{n}\right)\right)$. Thus $I_{n+1}$ is a pullback of $I_{n}$ with order at most $2 b^{2}+5 b(2 b-1)$. Note that if $c_{0}$ is a critical point of odd order, $I_{n}$ is never terminating and so we always have that $I_{n+1}=\Gamma^{T} \mathcal{B} \mathcal{A}\left(I_{n}\right)$. Finally, the interval $I_{n}$ is called Feigenbaum if it is periodic; necessarily of period two under $R_{I_{n-1}}$.

For each $n \geq 0$, let $r\left(I_{n}\right)$ be the minimal return time for $x \in I_{n} \cap \omega\left(c_{0}\right)$ back to $I_{n}$ and $\hat{r}\left(I_{n}\right)$ be the maximal entry time for $x \in \omega\left(c_{0}\right)$ to $I_{n}$.

Although the following was stated in $[\mathrm{KSvS}]$, we provide a proof, since the proof was not given in full in $[\mathrm{KSvS}]$ and the assumptions here are slightly weaker.

Lemma 2.3 (cf. [KSvS], Lemma 8.3). Assume that $I_{n}$ and $I_{n+1}$ are both non-terminating, then the following holds:

(1) $3 r\left(I_{n+1}\right) \geq p_{n}$,

(2) $\hat{r}\left(I_{n}\right) \leq\left(1 / 2^{5 b-1}\right) r\left(I_{n+1}\right)$.

Proof. For each $n \geq 0$ and for $0 \leq j \leq T-1$ define

$$
L_{n}=\mathcal{A}\left(I_{n}\right), \quad M_{n, 0}=K_{n}=\mathcal{B}\left(L_{n}\right) \quad \text { and } M_{n, j+1}=\Gamma\left(M_{n, j}\right) .
$$

Let $s_{n}$ and $t_{n}$ be such that $L_{n}$ is a pullback of $I_{n}$ under $f^{s_{n}}$ and $K_{n}$ is a pullback of $L_{n}$ under $f^{t_{n}}$. For each $0 \leq j \leq T-1$ let $q_{n, j}$ be such that $M_{n, j+1}$ is a pullback of $M_{n, j}$ under $f^{q_{n, j}}$. Finally define $p_{n}=s_{n}+t_{n}+q_{n, 1}+\cdots+q_{n, T}$. Using the same proof given in Lemma 8.2 on $[\mathrm{KSvS}$ ] we get

(i) $2 b^{2} r\left(I_{n+1}\right) \geq s_{n} \geq r\left(I_{n}\right)$,

(ii) $b^{2} r\left(K_{n}\right) \geq \bar{t}_{n} \geq r\left(L_{n}\right)$.

For each $j$ consider the chain $\left\{G_{j_{i}}\right\}_{i=0}^{q_{n, j}}$, with $G_{j_{n, j}}=M_{n, j-1}$ and $G_{j_{0}}=M_{n, j}$. By definition of $M_{n, j}$ we know that the interval $G_{j_{i}}$ does not contain $c_{0}$ if $0<i<q_{n, j}$. So we conclude that $r\left(M_{n, j}\right) \geq q_{n, j}$. The fact that $M_{n, j-1}$ is non-terminating for every $0 \leq j \leq T$ implies that $R_{M_{n, j-1}}\left(M_{n, j}\right) \cap M_{n_{j}}=\emptyset$. Therefore, $q_{n, j} \geq 2 r\left(M_{n, j-1}\right)$. Putting these two inequalities together we get

(iii) $r\left(M_{n, j}\right) \geq q_{n, j} \geq 2 r\left(M_{n, j-1}\right)$.

Since $M_{n, 1}$ is the smallest successor of $M_{n, 0}$ and $M_{n, 0} \subset I_{n}$ we have $\hat{r}\left(I_{n}\right) \leq \hat{r}\left(M_{n, 0}\right) \leq$ $q_{n, 1}$. Using this fact and equation (iii) we get

$$
\hat{r}\left(I_{n}\right) \leq q_{n, 1} \leq \frac{1}{2} q_{n, 2} \leq \cdots \leq \frac{1}{2^{T-1}} r\left(I_{n+1}\right) .
$$

The previous inequality together with equations (i) and (iii) gives us

$$
\begin{aligned}
p_{n} & =s_{n}+t_{n}+q_{n, 1}+\cdots+q_{n, T} \\
& \leq 2 b^{2} r\left(L_{n}\right)+b^{2} r\left(K_{n}\right)+q_{n, 1}+\cdots+q_{n, T} \\
& \leq 2 b^{2} r\left(K_{n}\right)+b^{2} r\left(K_{n}\right)+q_{n, 1}+\cdots+q_{n, T} \\
& \leq 2 b^{2} q_{n, 1}+b^{2} r q_{n, 1} / 2+q_{n, 1}+\cdots+q_{n, T}
\end{aligned}
$$




$$
\begin{aligned}
& \leq \frac{b^{2}}{2^{T-1}} r\left(I_{n+1}\right)+\frac{b^{2}}{2^{T-2}} r\left(I_{n+1}\right)+\left(\frac{1}{2^{T-1}}+\cdots+\frac{1}{2}\right) r\left(I_{n+1}\right) \\
& \leq \frac{3 b^{2}}{2^{T-2}} r\left(I_{n+1}\right)+\left(\frac{1}{2^{T-1}}+\cdots+\frac{1}{2}\right) r\left(I_{n+1}\right) \\
& \leq 3 r\left(I_{n+1}\right) .
\end{aligned}
$$

\section{Real Bounds Associated to the Enhanced Nest}

In this section we will consider maps $f \in \mathcal{A}_{b}^{3}$ and show geometric bounds for the intervals of the generalized enhanced nest Before stating the main result we need to introduce some terminology.

Given a constant $\rho>0$, a nice interval $I$ is called:

$$
\begin{array}{ll}
\rho \text {-nice } & \text { if for each } x \in I \cap \omega\left(c_{0}\right),(1+2 \rho) \mathcal{L}_{x}(I) \subset I ; \\
\rho \text {-free } & \text { if }\left((1+2 \rho) I \backslash(1+2 \rho)^{-1} I\right) \cap \omega\left(c_{0}\right)=\emptyset ; \\
\rho \text {-externally free } & \text { if there exists a nice interval } J \supset(1+2 \rho) I, \text { so that } J \supset I \text { is a } \\
& \text { nice pair and } J \cap \omega\left(c_{0}\right) \subset I ; \\
\rho \text {-internally free } & \text { if there exists a nice interval } J^{\prime}, \text { so that }(1+2 \rho) J^{\prime} \subset I, \\
& J^{\prime} \subset I \text { is a nice pair and } I \cap \omega\left(c_{0}\right) \subset J^{\prime} .
\end{array}
$$

We say that $I$ is $\rho$-strongly nice if it is $\rho$-nice and if for each $x, y \in I \cap \omega\left(c_{0}\right)$ either $\mathcal{L}_{x}(I)=\mathcal{L}_{y}(I),(1+2 \rho) \mathcal{L}_{x}(I) \cap \mathcal{L}_{y}(I)=\emptyset$ or $(1+2 \rho) \mathcal{L}_{y}(I) \cap \mathcal{L}_{x}(I)=\emptyset$. We will say that an interval $J$ is well-inside an interval $I$ if $(1+2 \rho) J \subset I$ for $\rho$ universal. If $\rho$ is large, then we say that $J$ is deep-inside $I$.

The purpose of this section is to prove the following theorem.

Theorem 3.1 (Real geometry of the enhanced nest). Suppose that $f \in \mathcal{A}_{\underline{b}}^{3}$. There exists $\varepsilon_{f}>0$ such that the following holds. Assume that $c_{0}$ is a critical point at which $f$ is persistently recurrent, and that either $c_{0}$ has even order or that every critical point in $\omega\left(c_{0}\right)$ has odd order. Suppose that $I_{0} \ni c_{0}$ is a nice interval with $\left|I_{0}\right|<\varepsilon_{f}$. Let $I_{0} \supset I_{1} \supset \cdots$ be the generalized enhanced nest for $f$ at $c_{0}$. Then the following hold:

(a) There exists $\rho>0$ such that if $I_{n}$ is non-terminating, then $I_{n}$ is $\rho$-nice. In addition, if $I_{n-1}$ is non-terminating then $I_{n}$ is $\rho$-externally and $\rho$-internally free, where the externally free space is given by an interval $J \supset(1+2 \rho) I_{n}$ and the internal free space is given by an interval $J^{\prime} \subset(1+2 \rho) J^{\prime} \subset I_{n}$. Moreover, if $c_{0}$ is even $\left|J^{\prime}\right| \geq \rho\left|I_{n}\right|$; if $c_{0}$ is odd then for each $v>0$ there exists $\rho^{\prime}>0$ so that if $\left|I_{n-1}\right| /\left|I_{n}\right|<v$ then $\left|J^{\prime}\right| \geq \rho^{\prime}\left|I_{n}\right|$.

(b) Suppose that $I_{n-1}$ is terminating and $I_{n}$ is non-terminating. Then for each $v>0$ there exists $\rho^{\prime}>0$ so that if $\left|I_{n-1}\right| /\left|I_{n}\right|,\left|I_{n}\right| /\left|I_{n+1}\right|<v$, then $I_{n}$ is $\rho^{\prime}$-free.

(c) For any $C^{\prime}>0$ there exists $C>0$ such that if $I_{n}$ is non-terminating and there is some $x \in \omega\left(c_{0}\right) \cap I_{n}$ with $(1+2 C) \mathcal{L}_{x}\left(I_{n}\right) \subset I_{n}$ then $\left(1+2 C^{\prime}\right) I_{n+1} \subset I_{n}$.

(d) For any $C^{\prime}>0$, there exists $C>0$ such that if $n \geq 0, I_{n}$ is non-terminating and $C$-nice, then $I_{n+1}$ is $C^{\prime}$-nice and $C^{\prime}$-externally free.

(e) Suppose that $I_{n}$ is non-terminating, then for each $\varepsilon^{\prime}>0$ there exists $\varepsilon>0$ such that if there is some $x \in \omega\left(c_{0}\right) \cap I_{n}$ with $\left|\mathcal{L}_{x}\left(I_{n}\right)\right| \leq \varepsilon\left|I_{n}\right|$, then $\left|I_{n+1}\right| \leq \varepsilon^{\prime}\left|I_{n}\right|$.

(f) For any $C^{\prime}>0$ there exists $\varepsilon>0$ so that if $I_{n}$ and $I_{n+1}$ are both non-terminating and $\left|I_{n+1}\right| \leq \varepsilon\left|I_{n}\right|$, then $I_{n+2}$ is a $C^{\prime}$-strongly nice and $C^{\prime}$-externally free. 
(g) For any $C^{\prime}>0$, there exists $C>0$ such that if $I_{n}$ is non-terminating, $I_{n+1}$ is terminating and $I_{n} \supset(1+2 C) I_{n+1}$, then $I_{n+1}^{\infty}$ is $C^{\prime}$-externally free.

Remark. (1) In the statements, the constants $C, C^{\prime}$ are large, while $\varepsilon, \varepsilon^{\prime}$ are small. This leads to intervals which are deep-inside, large free space, large ratios between intervals etc. For this reason, the bounds with $C, C^{\prime}$ and $\varepsilon, \varepsilon^{\prime}$ will be referred to as "big bounds".

(2) If $c_{0}$ is even, because of symmetry about the critical point, in Part (a) we have that $I_{n+1}$ is $\rho$-nice and $\rho$-free. Also, in Part (a) notice that if $\left|F^{\prime}\right| \geq \rho^{\prime}\left|I_{n}\right|$, then the components of $I_{n} \backslash F^{\prime}$ have size comparable to $I_{n}$. If $c_{0}$ is odd, then critical points can be close to the boundary of the return domains. This causes the map to lose free space inside the intervals. Because of this, the statement in Part (c) is no longer sufficient for our purposes, so we supplement it with (e).

(3) To get internal free space, in general, we require that the lengths of the intervals in few consecutive levels are comparable, see Corollary 3.25.

(4) In Part (a) the free space around $I_{n}$ is given directly by the construction of the enhanced nest, since return domains to $I_{n-1}$ are well-inside $I_{n-1}$. The return domains to $I_{n-1}$ can be close to the boundary, but when this happens they are very small. In case $I_{n-1}$ is terminating, the free space, when we have it, is a consequence of certain real bounds, rather than the construction of the enhanced nest.

Except for $\varepsilon_{f}$, which depends on $f$, the above bounds are universal, or in Sullivan's terminology beau. In other words, one can choose the constants in the above theorem so that they do not depend on $f$ but only on the vector $\underline{b}=\left(\ell_{1}, \ldots, \ell_{b}\right)$, provided we begin the construction with an interval $I_{0}$ small enough.

Theorem 3.1 generalizes Proposition 8.1 of [KSvS], to maps $f$ that are $C^{3}$ with critical points of any order (i.e. not necessarily even order) and allowing for maps which are possibly (infinitely) renormalizable. Proposition 3.20 is an addendum to Theorem 3.1 that will also be used later on. In the case of terminating intervals, some specific details of the proof of Proposition 3.20 will be frequently used throughout the paper.

An important difference between this work and $[\mathrm{KSvS}]$ is that we no longer get that

$$
\left(I_{n} \backslash(1+2 \rho)^{-1} I_{n}\right) \cap \omega\left(c_{0}\right)=\varnothing
$$

in the case where all critical points in $\omega(c)$ have odd order, which means that there can be small return domains to $I_{n}$ that intersect $\omega\left(c_{0}\right)$ close to the boundary of $I_{n}$. This complicates the proof significantly.

The proof of Theorem 3.1 occupies most of this section.

\subsection{Distortion bounds and their consequences.}

Theorem 3.2 ([vSV], Theorem C and [LiS], Theorem A). Suppose that $f \in \mathcal{A}_{\underline{b}}^{3}$. Then one has the following properties:

(1) Improved Macroscopic Koebe Principle. For each $\xi>0$, there exists $\xi^{\prime}>0$ such that if $I$ is a nice interval, $V$ is a nice interval that is $\xi$-well-inside $I, x \in I$ and $f^{k}(x) \in V$ with $k \geq 1$ not necessarily minimal, then the pullback of $V$ along $\left\{x, f(x), \ldots f^{k}(x)\right\}$ is $\xi^{\prime}$-well-inside the return domain to I containing $x$.

(2) Improved Koebe Principle. For each $\tau>0$, there exists $K>0$ and $\xi=\xi(f, \tau)>0$ satisfying the following. Let $T \subset M$ be an open interval, and let $J$ be a closed subinterval of $T$ such that the following hold: 
- $J \cap \omega\left(c_{0}\right) \neq \varnothing$,

- $f^{n} \mid T$ a diffeomorphism,

- $\left|f^{n}(T)\right|<\xi$,

- $f^{n}(J) \tau$-well-inside $f^{n}(T)$.

Then $f^{n} \mid J$ has bounded distortion; that is, for any $x, y \in J$,

$$
\frac{\left|D f^{n}(x)\right|}{\left|D f^{n}(y)\right|} \leq K \text {. }
$$

Furthermore, $K \rightarrow 1$ as $\tau \rightarrow \infty$.

(3) Negative Schwarzian derivative. For each critical point c that is not in the basin of a periodic attractor, there exists a neighbourhood $U$ of $c$ such that whenever $f^{n}(x) \in U$ for some $x \in I$ and $n \geq 0$, the Schwarzian derivative of $f^{n+1}$ at $x$ is negative.

Theorem 3.2(2) follows from [LiS, Theorem A]. There the result is stated for mappings with all periodic orbits repelling. However, the Theorem holds, without any change, if we consider $f$ restricted to puzzle pieces intersecting $\omega\left(c_{0}\right)$; since all periodic points contained in those pieces are repelling.

From now on we assume the following

\section{Standing Assumptions:}

- $f \in \mathcal{A}_{\underline{b}}^{3}$ is persistently recurrent on $\omega(c)$, where $c \in \operatorname{Crit}(f)$.

- Either $c_{0} \in \omega(c)$ is even or $c_{0}$ is odd and every critical point in $\omega(c)$ is odd.

- Any nice interval $I$ that intersects $\omega\left(c_{0}\right)$ is so small that for any chain $\left\{G_{j}\right\}_{j=0}^{s}$ with $G_{s}=I$ and $G_{0} \cap \omega(c) \neq \varnothing$ each $G_{j}$ avoids a neighbourhood of any immediate basin of attraction of any periodic attractor or any parabolic cycle.

- If $I$ is a nice interval containing a critical point $c^{\prime} \in \omega(c)$, then $I$ is so small that the remark on p. 20 holds for $I$.

- Suppose $I$ and $J$ are nice intervals with $(1+2 \delta) J \subset I$ with $\delta>0$ universal. We assume $|I|<\xi$, where $\xi=\min \left\{\xi(f, \tau): \delta \leq \tau \leq 10^{10}\right\}$, and $\xi(f, \tau)$ is the constant given by Theorem 3.2 (2).

We can assume these since, as we have seen in Sect. 2.3, we can guarantee that puzzle pieces intersecting $\omega\left(c_{0}\right)$ are arbitrarily small. It is worth observing that if $(1+2 C) J \subset I$ for $C$ large, then taking $J^{\prime}=(1+2(C-1 / 2)) J$, we can apply the Improved Koebe Principal with $\xi=\xi(f, 1 / 2)>0$, so that when $C$ is large our control on the distortion given by Theorem 3.2 remains bounded.

Fact 9.1 of [KSvS] does not generalize to our present setting (the proof in [KSvS] relies on the maps having negative Schwarzian derivative). However, the following analogue holds.

Lemma 3.3 [KSvS, Fact 9.1]. For each $N \in \mathbb{N}$ and $\rho>0$ there exists $\rho^{\prime}>0$ such that the following holds. Let $\left\{G_{j}\right\}_{j=0}^{s}$ and $\left\{G_{j}^{\prime}\right\}_{j=0}^{s}$ be chains such that $G_{j} \subset G_{j}^{\prime}$ for all $j$, $0 \leq j \leq s$ and $G_{0} \cap \omega\left(c_{0}\right) \neq \emptyset$. Assume that the order of $\left\{G_{j}^{\prime}\right\}_{j=0}^{s}$ is at most $N$ and that $(1+2 \rho) G_{s} \subset G_{s}^{\prime}$. Then $\left(1+2 \rho^{\prime}\right) G_{0} \subset G_{0}^{\prime}$. Furthermore, for fixed $N, \rho^{\prime} \rightarrow \infty$ as $\rho \rightarrow \infty$.

Proof. Let $\hat{G}_{s}=(1+\rho) G_{s}$ and consider the chain $\left\{\hat{G}_{j}\right\}_{j=0}^{s}$ with $G_{j} \subset \hat{G}_{j}$. Observe that $\hat{G}_{s}$ is $\rho / 2$-well-inside of $G_{s}^{\prime}$. Let $0<s_{1}<\cdots<s$ be the times $j$ so that $G_{j}^{\prime}$ 
contains a critical point. Then we can decompose $f^{s}: G_{0}^{\prime} \rightarrow G_{s}^{\prime}$ into at most $N$ maps of the form $f: G_{s_{j-1}}^{\prime} \rightarrow G_{s_{j-1}+1}^{\prime}$ followed by $f^{s_{j}-s_{j-1}-1}: G_{s_{j-1}+1}^{\prime} \rightarrow G_{s_{j}}^{\prime}$. It follows from Theorem 3.2(2) that each of the factors $\left.f^{s_{j}-s_{j-1}+1}\right|_{\hat{G}_{s_{j-1}+1}}$ has bounded distortion. This, along with the fact that $f$ has non-flat critical points, implies that existence of $\rho^{\prime}>0$ so that $\left(1+2 \rho^{\prime}\right) G_{0} \subset \hat{G}_{0} \subset G_{0}^{\prime}$.

Two immediate consequences of the previous two results are:

Corollary 3.4. For each $\rho>0$ there exists $\rho^{\prime}>0$ so that the following holds. If $I$ is a nice interval, $J=\mathcal{L}_{x}(I)$ for some $x \in \omega\left(c_{0}\right)$ and $(1+2 \rho) J \subset I$ then, $J$ is $\rho^{\prime}$-nice. Furthermore, if I is $\rho$-nice then $\rho^{\prime} \rightarrow \infty$ as $\rho \rightarrow \infty$.

Corollary 3.5. For each $\rho>0$ and each integer $N \geq 0$, there exists $\rho^{\prime}>0$ so that the following holds. Let I and $J$ be nice intervals with $J$ a pullback of I of order bounded by $N$ that intersects $\omega\left(c_{0}\right)$. Then if I is $\rho$-free, $J$ is $\rho^{\prime}$-free. Furthermore, for fixed $N$, we get that $\rho^{\prime} \rightarrow \infty$ as $\rho \rightarrow \infty$.

Observe the above implies that if $I$ is $\rho$-externally free then $J$ is $\rho^{\prime}$-externally free; however, we do not claim that if there exists $\rho>0$ such that $\left(I \backslash(1+2 \rho)^{-1} I\right) \cap \omega\left(c_{0}\right)=\emptyset$, then there exists $\rho^{\prime}>0$ such that $\left(J \backslash\left(1+2 \rho^{\prime}\right)^{-1} J\right) \cap \omega\left(c_{0}\right)=\varnothing$. This statement may be wrong, because the internal free space that we pull back may be small compared to the total interval $J$, unless we also have external free space to control the distortion of the mapping.

The next two lemmas are closely related and are useful when a return domain to $I$ is not well-inside $I$.

Lemma 3.6 [vSV, Lemmas 2 and 3]. There exists a constant $\rho=\rho(\underline{b})>0$ with the following property. Let I be a nice interval, consider a point $x \in I$ which returns to I in time $s$ and let $I^{1}=\mathcal{L}_{x}(I)$. Then either of the following holds:

(1) $(1+2 \rho) I^{1} \subset I$,

(2) there exists an interval $G_{s} \supset(1+2 \rho) I^{1}$ with $I \subset G_{s}$, so that the chain $\left\{G_{j}\right\}_{j=0}^{s}$ with $G_{0} \ni x$ has intersection multiplicity bounded from above by a constant $N$ that depends only of the modality of $f$.

3.2. The existence of suitable fundamental domains. Let $J \subset J^{\prime}$ be intervals and assume that $g: J \rightarrow J^{\prime}$ is monotone. An interval $J^{*}$ is called a fundamental domain for $g$ if it is of the form $(x, g(x))$ (or $(g(x), x)$ ). We will repeatedly use the property that the pullbacks of a fundamental domain under iterates of $g$ are disjoint.

Lemma 3.7. For each $\sigma \in(0,1)$ there exist $\kappa>0$ and for each $f \in \mathcal{A}_{\underline{b}}^{3}$ there exists an integer $N$ such that the following holds. Let I be a nice interval and let $J$ be a first return domain of I with return time $n>N$. Assume that $f^{n}: J \rightarrow I$ is an orientation preserving diffeomorphism with fixed point $p$ and let $J^{\prime}$ be a component of $J \backslash\{p\}$. Then for each $x \in J^{\prime}$ with $|x-p| \geq \sigma\left|J^{\prime}\right|$ there exists a fundamental domain $F$ containing $x$ with $|F| \geq \kappa \cdot d(F, p)$.

Proof. By Theorem IV.B in [dMvS] there exists $\delta_{1}>0$ so that for each $f \in \mathcal{A}_{b}^{3}$ there exists $N$ so that $D f^{n}(p) \geq 1+\delta_{1}$ provided the period $n$ of $p$ is at least $N$. For simplicity 
assume that $p=0$ and that $[0, x] \subset J^{\prime}$. Write $l=\{0\}, j=[0, \sigma x], r=[\sigma x, x]$, $t=[0, x]$ and $g=f^{n}$. Define the cross-ratio distortion

$$
B(g, t, j)=\frac{|g(j)||g(t)|}{|g(l)||g(r)|} \cdot \frac{|l||r|}{|t||j|} .
$$

Take $y=\sigma x$ and take $\delta>0$ so that $g(\sigma x) /(\sigma x)=1+\delta$. Then

$$
B(g, t, j)=\frac{1}{g^{\prime}(0)} \frac{g(\sigma x)}{(\sigma x)} \frac{g(x)}{x} \frac{x-\sigma x}{g(x)-g(\sigma x)} \leq \frac{1+\delta}{1+\delta_{1}} \frac{1-\sigma}{1-\sigma(1+\delta),}
$$

since $g(x) \geq x$. We would like to show that $\delta$ uniformly bounded from below for all $x \in J^{\prime}$. If this is not the case, taking $\delta>0$ small we get $B(g, t, j)<1$ contradicting Theorem 2.1 of Chapter 4 of $[\mathrm{dMvS}]$; which states that $B(g, t, j) \geq 1$.

3.3. The principal nest. Recall that if $I$ is a nice interval that contains a recurrent point $x$, then the principal nest about $x$ is defined inductively by $I^{0}=I$ and $I^{n+1}=\mathcal{L}_{x}\left(I^{n}\right)$. The next lemma is an important bound on the geometry of intervals in the principal nest.

Lemma 3.8 [vSV, Theorem A]. There exists $\delta>0$ such that if $I$ is a nice interval, $x \in I$ is recurrent and $R_{I}(x) \notin \mathcal{L}_{x}(I)$, then for each $d \in \mathbb{N}$ if $R_{\mathcal{L}_{x}^{d}(I)}(x) \notin \mathcal{L}_{x}^{d+1}(I)$, then

$$
(1+2 \delta) \mathcal{L}_{x}^{d+2}(I) \subset \mathcal{L}_{x}^{d+1}(I) .
$$

Lemma 3.9 [S3, Lemma 5.5]. There exists a constant $\rho=\rho(\underline{b})>0$ with the following property. Let I be a periodic interval of sufficiently large period s. Then $(1+2 \rho) I$ does not contain $f^{i}(I)$ for $i=1, \ldots, s-1$. Moreover, the chain $\left\{G_{j}\right\}_{j=0}^{s}$ with $G_{s}=(1+2 \rho) I$ and $G_{0} \supset I$ has the following two properties

- $\left\{G_{j}\right\}_{j=0}^{s}$ has intersection multiplicity at most four and

- the map $f^{s}$ does not have a critical point in $G_{0} \backslash I$.

We say that $I:=I^{0} \supset I^{1} \supset \cdots \supset I^{m}$ is a central cascade if $m \geq 2$ and the return time of $I^{m}$ to the intervals $I^{0}, I^{1}, \ldots, I^{m-1}$ is always the same.

Lemma 3.10 (cf. [KSvS], Lemma 9.5). For any $\delta>0$ there exist $\kappa>0$ and $C>0$ with the following properties. Let I be a nice interval (as usual, assumed to be sufficiently small), having a central cascade $I:=I^{0} \supset I^{1} \supset \cdots \supset I^{m}$ with $m \geq 2$ and let $r$ be the return time of $I^{1}$ to $I^{0}$.

(1) If $\left|I^{2}\right| \geq \delta\left|I^{0}\right|$, then for any critical point c of the map $R_{I} \mid I^{2}$ we have

$$
\left|f^{r}(c)-c\right| \geq \kappa\left|I^{0}\right| \text { and }\left|D f^{r}(x)\right| \leq C \text { for all } x \in I^{2} \text {. }
$$

(2) If $\left|I^{1}\right| \geq \delta\left|I^{0}\right|$ and we let $\tilde{I}=(1+2 \delta) I$ and $\tilde{I}^{1}=\operatorname{Comp}_{I^{1}}\left(f^{-r}(\tilde{I})\right)$ the following holds. Suppose that $f^{r}$ extends to a map from $\tilde{I}^{1}$ to $\tilde{I}$, with the same set of critical points as $f^{r} \mid I^{1}$, that can be decomposed into a finite composition of maps with bounded distortion and polynomials. Then for any critical point c of the map $R_{I} \mid I^{1}$ we have

$$
\left|f^{r}(c)-c\right| \geq \kappa\left|I^{0}\right|, \text { and }\left|D f^{r}(x)\right| \leq C \text { for all } x \in I^{1} \text {. }
$$


Proof. As before, let $\underline{b}=\left(\ell_{1}, \ldots, \ell_{b}\right)$. By Lemma 3.6; Theorem 3.2 and since $c \in I^{2}$, there exist beau constants $\rho^{\prime}>0, K<\infty$ and an integer $N$ (only depending on $\underline{b}$ ) and an interval $J$ with either $J=I^{2}$ or with $J$ of the form $(c-\delta, c+\delta)$ so that $|J| \geq \bar{\rho}^{\prime}\left|I^{2}\right|$, $f^{r}(J) \subset\left(1+\rho^{\prime}\right) I_{0}$ and so that $f^{r}: J \rightarrow f^{r}(J)$ can be written as a composition of at most $N$ maps whose distortion is bounded by $K$ and polynomials $z \mapsto z^{\ell_{i}}$. Taking $J=I^{2}$ the 2 nd inequality in (1) follows. To prove the 1 st inequality, take $J$ as above of the form $(c-\delta, c+\delta)$. So there exists a beau constant $\hat{K}<\infty$ so that for all $\epsilon \in(0,1)$ one has $|D f(x)| \leq \epsilon \hat{K}|f(J)| /|J|$ for all $x \in(c-\epsilon \delta, c+\epsilon \delta)$ and $\left|D f^{r-1}(y)\right| \leq$ $\hat{K}^{N-1}\left|f^{r}(J)\right| /|J|$ for all $y \in f(J)$. It follows that $\left|D f^{r}(x)\right| \leq \epsilon \hat{K}^{N}\left|f^{r}(J)\right| /|J|$ for all $x \in(c-\epsilon \delta, c+\epsilon \delta)$ and $\left|f^{r}(J)\right| /|J| \leq\left(\left(1+\rho^{\prime}\right) / \rho^{\prime}\right)\left|I_{0}\right| /\left|I_{2}\right| \leq\left(\left(1+\rho^{\prime}\right) /\left(\delta \rho^{\prime}\right):=K^{*}\right.$. If we take $\epsilon>0$ so that $\epsilon \hat{K}^{N} K^{*}<1 / 2$ then $\left|D f^{r}(x)\right| \leq(1 / 2)$ for all $x \in(c-\epsilon \delta, c+\epsilon \delta)$. Now choose $\kappa=\epsilon /\left(16 \delta \rho^{\prime}\right)$ and assume by contradiction that $\left|f^{r}(c)-c\right| \leq \kappa\left|I^{0}\right|$. Then $\left|f^{r}(c)-c\right| \leq\left(\epsilon /\left(16 \delta \rho^{\prime}\right)\left|I^{0}\right| \leq \epsilon /\left(16 \rho^{\prime}\right)\left|I^{2}\right| \leq(\epsilon / 16)|J|=\epsilon \delta / 8\right.$. Thus we get that $f^{r}$ maps $(c-\epsilon \delta, c+\epsilon \delta)$ into itself and $\left|D f^{r}(x)\right| \leq 1 / 2$ on this interval. Hence $f^{r}$ has an attracting fixed point, which contradicts that $\omega(c)$ is minimal. Thus we have proved the 1 st inequality in (1) by contradiction. The inequalities in (2) follow as in (1).

\subsection{Geometry of pullbacks.}

Lemma 3.11. Let I be a nice interval. Assume $z^{\prime} \notin I$ has first entry time to I equal to $k>0$ and that there exists $l>k$ such that $f^{l}\left(z^{\prime}\right) \in I$. Letting $z=f^{k}\left(z^{\prime}\right), I^{1}=\mathcal{L}_{z}(I)$, $\hat{I}=\mathcal{L}_{z^{\prime}}(I)$ and $\hat{K}=\mathcal{L}_{z^{\prime}}(\hat{I})$, we have that $\hat{K} \subset \mathcal{L}_{z^{\prime}}\left(I^{1}\right)$.

Proof. Since $\hat{I}$ is a pullback of $I$ and $\hat{K}$ is a pullback of $\hat{I}, \hat{K}$ is a pullback of $I$, by say $f^{s}$. Since $k$ is the first entry time of $z^{\prime}$ to $I$ we have that $k<s$. Let $K^{\prime}=\operatorname{Comp}_{z} f^{-(s-k)}(I)$. Since $z^{\prime} \in \hat{K}, z \in K^{\prime}$ and $I^{1}$ is a pullback of $I$ containing $z$, we have that $K^{\prime} \subset I^{1}$. Hence $\hat{K} \subset \mathcal{L}_{z^{\prime}}\left(I^{1}\right)$.

Lemma 3.12 (cf. [S3] Proposition 4.1). For any $C>0$ and any $d \in \mathbb{N}$, there exists $C^{\prime}>0$ so that the following holds. Assume $I \supset J$ are nice intervals with nested or disjoint pullbacks and $J \supset \mathcal{L}_{x}^{d}(I)$ for some $x \in \omega\left(c_{0}\right)$. If $\left(1+2 C^{\prime}\right) J \subset I$, then $(1+2 C) \mathcal{L}_{y}(J) \subset \mathcal{L}_{y}(I)$ for any $y \in \omega\left(c_{0}\right)$.

If $c_{0}$ is odd, then for any $\delta>0$ and $C>0$ there is exists $C^{\prime}>0$ so that the following holds. Let $I$ be a nice interval with $((1+2 \delta) I \backslash I) \cap \omega\left(c_{0}\right)=\emptyset$. Suppose that $J \subset I$ is a nice interval with $J \supset \mathcal{L}_{x}(I)$ for some $x \in \omega\left(c_{0}\right)$. If $|J| \leq|I| /\left(1+2 C^{\prime}\right)$ then $\left|\mathcal{L}_{y}(J)\right| \leq\left|\mathcal{L}_{y}(I)\right| /(1+2 C)$ for any $y \in \omega\left(c_{0}\right)$.

Proof. We will prove the first part of the lemma by induction on $d$. Let us begin with the case $d=1$. In [vSV, Theorem B2], the result is stated for $J=\mathcal{L}_{c}(I)$, but the proof holds for $J \supset \mathcal{L}_{c}(I)$ provided $J, I$ are a nice pair (definition p.18). The fact that $d=1$ implies that $I$ and $J$ are a nice pair so the result follows. Before we prove the general case, we observe that the constant $C^{\prime}$ will depend on $d$. Assume the result holds for all $d^{\prime}<d$ and let us show it for $d$. Since $y \in \omega\left(c_{0}\right)$, there exists $s>0$, minimal, so that $z=f^{s}(y) \in J$. Consider the chains $\left\{G_{j}\right\}_{j=0}^{s}$ with $G_{s}=J$ and $G_{0}=\mathcal{L}_{y}(J)$ and $\left\{G_{j}^{\prime}\right\}_{j=0}^{s}$ with $G_{s}^{\prime}=I$ and $G_{0}^{\prime}=\operatorname{Comp}_{y} f^{-s}(I)$. Let $0<s^{\prime}<s$ be maximal so that $f^{s^{\prime}}(y) \in I$. If there exists no such $s^{\prime}$, then $z$ is the first entry time of $y$ to both $I$ and $J$, so the chain $\left\{G_{j}^{\prime}\right\}_{j=1}^{s}$ is disjoint, therefore it has order bounded by $b$. In this case the result follows from Lemma 3.3. If $s^{\prime}$ is defined, let $z^{\prime}=f^{s^{\prime}+1}(y)$ and $\hat{I}=G_{s^{\prime}+1}^{\prime}$. Observe 
$\hat{I}=\mathcal{L}_{z^{\prime}}(I)$. Since $I$ is small and it intersects $\omega\left(c_{0}\right)$ the entry time of $f^{s^{\prime}}(y)$ to $I$ is bigger than one, so $z^{\prime} \notin I$. Let $J^{\prime}=G_{s^{\prime}+1}$. By the definition of $s^{\prime}$, the chain $\left\{G_{j}^{\prime}\right\}_{j=s^{\prime}+1}^{s}$ is disjoint, so Lemma 3.3 implies that $J^{\prime}$ is deep-inside $\hat{I}$. Observe that $\mathcal{L}_{z}^{i}(I)=\mathcal{L}_{x}^{i}(I)$ for $i<d$ since $z \in J$. From Lemma 3.11 we conclude that $J^{\prime} \supset \mathcal{L}_{z^{\prime}}^{d-1}(\hat{I})$, and the result follows from the induction hypothesis.

The proof of the 2 nd part of the lemma is fairly involved and will be given in the next subsection.

3.4.1. Proof of the 2 nd part of Lemma 3.12. If $I$ is a nice interval that contains a recurrent point $x$, then define

$$
\mathcal{C}_{x}(I):=\left\{\begin{array}{cl}
I^{m} & \text { if } I \text { is non-terminating and } \\
I^{\infty} & \text { otherwise, }
\end{array}\right.
$$

where $m$ is minimal such that $R_{I}(x) \notin I^{m}$ We will sometimes omit the $x$ from the notation when it will not cause confusion.

By [vSV, Lemma 15], if $U \subset I$ is a nice interval and $U \cap \mathcal{L}_{x}(I)=\emptyset$, then any pullback of $U$ that contains $x$ is contained in $\mathcal{C}_{x}(I)$.

Lemma 3.13 (cf. [vSV], Lemma 16). Let $\delta>0$ and suppose that $c_{0}$ is odd (so every critical point in $\omega\left(c_{0}\right)$ is odd). There exists a function $\rho_{1}: \mathbb{R}^{+} \rightarrow \mathbb{R}^{+}$such that $\rho_{1}(\varepsilon) \rightarrow 0$ as $\varepsilon \rightarrow 0$ with the following properties. Let $I^{0}$ be a nice interval containing a point $c \in \omega\left(c_{0}\right)$ with $\left((1+2 \delta) I^{0} \backslash I^{0}\right) \cap \omega\left(c_{0}\right)=\emptyset$. Let $V$ be an interval such that $|V| /\left|I^{0}\right|<\varepsilon$ and such that $V \subset I^{0}$ is a nice pair. Suppose that $f^{s}(x) \in V$ for some $s>0$ and some $x \in I^{0} \cap \omega\left(c_{0}\right)$. Let $G_{s}=V, \hat{G}_{s}=I^{0}$ and let $\left\{G_{j}\right\}_{j=0}^{s},\left\{\hat{G}_{j}\right\}_{j=0}^{s}$ be the chains so that

$$
\hat{G}_{i}=\operatorname{Comp}_{f^{i}(x)}\left(f^{-(s-i)}\left(\hat{G}_{s}\right)\right) \text { and } G_{i}=\operatorname{Comp}_{f^{i}(x)}\left(f^{-(s-i)}\left(G_{s}\right)\right) .
$$

Then there exist $t, 0 \leq t<s$ and an interval $\hat{G}_{t}^{1}$ with $G_{t} \subset \hat{G}_{t}^{1} \subset \mathcal{L}_{f^{t}(x)}\left(I^{0}\right)$ such that

$$
\frac{\left|G_{t}\right|}{\left|\hat{G}_{t}^{1}\right|}<\rho_{1}(\varepsilon) .
$$

If $t>0$, then:

(1) $G_{t} \subset \hat{G}_{t}^{1}$ is a nice pair and

(2) each pullback of $\hat{G}_{t}^{1}$ that intersects $c$ is contained in $\mathcal{C}_{c}\left(I^{0}\right)$.

Proof. Let $I^{1}=\mathcal{L}_{c}\left(I^{0}\right)$. Let $\phi: I^{1} \rightarrow I^{0}$ be the first return map of $I^{1}$ to $I^{0}$. Since $\left((1+2 \delta) I^{0} \backslash I^{0}\right) \cap \omega\left(c_{0}\right)=\varnothing$, by the Improved Koebe Principle, Theorem 3.2(2), any first entry map to $I^{0}$ decomposes into at most $b$ maps of the form $z \mapsto z^{\ell}$, where $\ell$ is odd, followed by a diffeomorphism with bounded distortion. Hence, there exists a function $\hat{\rho}: \mathbb{R}^{+} \rightarrow \mathbb{R}^{+}$with $\hat{\rho}(\varepsilon) \rightarrow 0$ as $\varepsilon \rightarrow 0$ so that any pullback, $V^{\prime}$, of $V$ by a first entry map to $I_{0}$ satisfies

$$
\frac{\left|V^{\prime}\right|}{\left|\mathcal{L}_{V^{\prime}}\left(I^{0}\right)\right|}<\hat{\rho}(\varepsilon) .
$$

It is worth noticing that this is where we use the fact that all critical points in $\omega\left(c_{0}\right)$ are odd. 
Let $0=: t_{0}<t_{1}<\cdots<t_{k}:=s$ be the integers such that $f^{t_{j}}(x) \in I^{0}$. By the preceding comment,

$$
\frac{\left|G_{t_{k-1}}\right|}{\left|\hat{G}_{t_{k-1}}\right|}=\frac{\left|G_{t_{k-1}}\right|}{\left|\mathcal{L}_{f^{t_{k-1}(x)}}\left(I^{0}\right)\right|}<\hat{\rho}(\varepsilon) .
$$

Hence, if $f^{t_{k-1}}(x) \notin I^{1}$, then all required properties hold for $t=t_{k-1}$ taking $\hat{G}_{t}^{1}=$ $\mathcal{L}_{f^{t}(x)}\left(I^{0}\right)$ and $\rho_{1}=\hat{\rho}$, so from now on we assume that $f^{t_{k-1}}(z) \in I^{1}$. Now let $k^{\prime}$ be minimal such that

$$
f^{t_{k^{\prime}}}(x), f^{t_{k^{\prime}+1}}(x), \ldots, f^{t_{k-1}}(x) \in I^{1}
$$

Since $R_{I^{0}} \mid I^{1}$ is monotone, we have that $\hat{G}_{t_{k^{\prime}}} \subset \hat{G}_{t_{k^{\prime}+1}} \subset \cdots \subset \hat{G}_{t_{k-1}}=I^{1} \subset \hat{G}_{t_{k}}=I^{0}$. We can assume that $\frac{\left|I^{1}\right|}{\left|I^{0}\right|}$ is not small: otherwise, if $k^{\prime}>0$, the required properties hold for $t=t_{k^{\prime}-1}$ and $\hat{G}_{t}^{1}=\mathcal{L}_{f^{t}(x)}\left(I^{0}\right)$, and if $k^{\prime}=0$, we set $t=0$ and $\hat{G}_{t}^{1}=I^{1}$. This means that we can additionally assume that $V \subset I^{0} \backslash I^{1}$ : since $V \subset I^{0}$ is a nice pair, if this was not the case, we would have $I^{1} \subset V$, but then $I^{1}$ would be very small compared to $I^{0}$.

Claim. There exists a function $\rho_{1}$, as above such that

$$
\frac{\left|G_{k^{\prime}}\right|}{\left|I^{1}\right|} \leq \rho_{1}(\varepsilon) .
$$

Proof of claim. We will assume that $\phi: I^{1} \rightarrow I^{0}$ is orientation preserving (if it is not, we replace it by its second iterate). Let $p$ be a fixed point of $\phi: I^{1} \rightarrow I^{0}$, let $\hat{G}_{t_{i}, \pm}$ denote the components of $\hat{G}_{t_{i}} \backslash\{p\}$ where $\hat{G}_{t_{i},+}$ is on the same side of $p$ as $V$.

Case 1. If the union of two adjacent fundamental domains, $\hat{G}_{t_{k},+} \backslash \hat{G}_{t_{k-2},+}$ is much bigger than $V$, then we are done since the pullback of $\hat{G}_{t_{k},+} \backslash \hat{G}_{t_{k-2},+}$ under the first return map to $I^{1}$ has intersection multiplicity bounded by three.

Case 2. The condition of Case 1 does not hold, but $\left|\hat{G}_{t_{k-1},+} \backslash \hat{G}_{t_{k-2},+}\right|$ is much bigger than $\left|\hat{G}_{t_{k-2}}\right|$. Then $G_{t_{i}}$ is much smaller than $I_{0}$ for all $i=k^{\prime}, \ldots, k$. Using this statement for $i=k^{\prime}+1$ and pulling back once more we are done.

Case 3. $\left|\hat{G}_{t_{k-1},+} \backslash \hat{G}_{t_{k-2},+}\right|$ is much smaller than $\left|\hat{G}_{t_{k-2},+}\right|$. By the decomposition of the return map into maps with bounded distortion followed by a polynomial, the derivative of $\phi: \hat{G}_{t_{k-2}} \rightarrow I^{1}$ is bounded. Hence, since $G_{t_{k-1}}$ is very small in $I^{1}$ and $G_{t_{k-1}} \subset$ $\hat{G}_{t_{k-1}} \backslash \hat{G}_{t_{k-3}}$, we have that $\left|G_{t_{k-1}}\right|$ is very small compared to $\left|\hat{G}_{t_{k-1}} \backslash \hat{G}_{t_{k-3}}\right|$. The proof now follows as in Case 1 .

To complete the proof of the lemma, if $k^{\prime}>0$, take $t=t_{k^{\prime}-1}$ and $\hat{G}_{t}^{1}=\mathcal{L}_{f^{t}(x)}\left(I^{0}\right)$, and if $k^{\prime}=0$, take $t=0$ and $\hat{G}_{t}^{1}=\mathcal{L}_{x}\left(I^{1}\right)$.

We now prove the second part of Lemma 3.12. 
Lemma 3.14 (cf. [vSV] Proposition 5). Suppose $c_{0}$ is odd. Assume that I $\supset$ are nice intervals such that their pullbacks are either nested or disjoint, with $((1+2 \delta) I \backslash I) \cap$ $\omega\left(c_{0}\right)=\emptyset$. Let $y \in \omega\left(c_{0}\right) \cap I$. Then for any $\varepsilon>0$, there exists $\varepsilon^{\prime}>0$ such that if $J \supset \mathcal{L}_{y}(I)$ and

$$
\frac{|J|}{|I|}<\varepsilon^{\prime},
$$

then for any $x \in \omega\left(c_{0}\right)$,

$$
\frac{\left|\mathcal{L}_{x}(J)\right|}{\left|\mathcal{L}_{x}(I)\right|}<\varepsilon
$$

Proof. Notice that if $I \supset J \supset \mathcal{L}_{x}(I)$ and $I$ and $J$ are nice intervals with nested or disjoint pullbacks, then $I \supset J$ is a nice pair.

Let $s$ be the entry time of $x$ to $J$. Let $\hat{G}_{s}^{0}=I$ and $G_{s}=J$, and define chains $\left\{\hat{G}_{i}^{0}\right\}_{i=0}^{s}$ and $\left\{G_{i}\right\}_{i=0}^{s}$ so that $G_{i} \subset \hat{G}_{i}^{0}$ are the pullbacks of $G_{s} \subset \hat{G}_{s}^{0}$ containing $f^{i}(x)$. Let $s_{1}$ be maximal so that $\hat{G}_{s_{1}}^{0}$ contains a critical point of $f$. If no such $s_{1}$ exists, we set $s_{1}=0$. Then by the Improved Koebe Principle, there exists a function $\hat{\rho}: \mathbb{R}^{+} \rightarrow \mathbb{R}^{+}$ with $\hat{\rho}(\varepsilon) \rightarrow 0$ as $\varepsilon \rightarrow 0$ such that

$$
\frac{\left|G_{s_{1}}\right|}{\left|\hat{G}_{s_{1}}^{0}\right|}<\hat{\rho}(\varepsilon) .
$$

If $s_{1}=0$, then the proof is finished. If $s_{1}>0$, let $c_{1}$ be the critical point in $\hat{G}_{s_{1}}^{0}$. Note that $\hat{G}_{s_{1}}^{0} \supset G_{s_{1}}$ is a nice pair. Now let $t_{1}$ and $\hat{G}_{t_{1}}^{1}$ be the time and the interval given by the previous lemma. Then

$$
\frac{\left|G_{t_{1}}\right|}{\left|\hat{G}_{t_{1}}^{1}\right|} \leq \rho_{1} \circ \hat{\rho}(\varepsilon) .
$$

If $t_{1}=0$, this completes the proof. Otherwise, $G_{t_{1}} \subset \hat{G}_{t_{1}}^{1}$ is a nice pair, and moreover, any pullback of $\hat{G}_{t_{1}}^{1}$ that intersects $c_{1}$ is contained in $\mathcal{C}_{c_{1}}\left(\hat{G}_{s_{1}}^{0}\right)$.

Repeating this construction inductively, we obtain a sequence of times $t_{0} \geq s_{1}>$ $t_{1}>\cdots>s_{\kappa-1}>t_{\kappa-1} \geq s_{\kappa} \geq t_{\kappa}=0$ and a sequence of nice pairs $G_{t_{i}} \subset \hat{G}_{t_{i}}^{i}$, $i=0,1, \ldots, \kappa-1$ such that

(1) for $i=1, \ldots, \kappa,\left|G_{s_{i}}\right| /\left|\hat{G}_{s_{i}}^{i}\right|<\hat{\rho}\left(\left(\rho_{1} \circ \hat{\rho}\right)^{i-1}(\varepsilon)\right)$;

(2) for $i=0,1, \ldots, \kappa,\left|G_{t_{i}}\right| /\left|\hat{G}_{t_{i}}^{i}\right|<\left(\rho_{1} \circ \hat{\rho}\right)^{i}(\varepsilon)$;

(3) if $\hat{G}_{t_{i}}^{i}, \hat{G}_{t_{j}}^{j}$, with $i<j$, both contain the same critical point $c$, then $\hat{G}_{t_{j}}^{j} \subset \mathcal{C}_{c}\left(\hat{G}_{t_{i}}^{i}\right)$.

If $\kappa$ is not large, the proposition follows immediately. If $\kappa$ is large, we use a different argument. By the last property, there is a critical point $c$ and a sequence $i(1)<i(2)<$ $\cdots<i(r)$, with $r>\kappa /(b-1)$, such that

$$
\hat{G}_{n_{i(1)}}^{i(1)} \supset \mathcal{C}_{c}\left(\hat{G}_{\left.n_{i(1)}^{i(1)}\right)}\right) \hat{G}_{n_{i(2)}^{i(2)}}^{{ }_{1}} \supset \mathcal{C}_{c}\left(\hat{G}_{n_{i(2)}}^{i(2)}\right) \supset \cdots \supset \hat{G}_{n_{i(r)}}^{i(r)} \supset G_{n_{i(r)}} .
$$

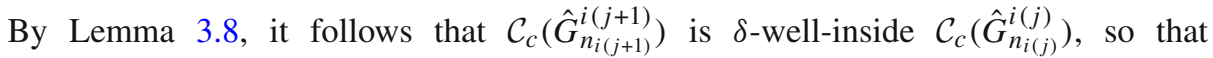
$\mathcal{L}_{x}\left(\mathcal{C}_{c}\left(\hat{G}_{n_{i(j+1)}}^{i(j+1)}\right)\right.$ is $\delta^{\prime}$-well-inside $\mathcal{L}_{x}\left(\mathcal{C}_{c}\left(\hat{G}_{n_{i(j)}}^{i(j)}\right)\right)$. Since this holds for $j=1, \ldots, r-1$, 
$\mathcal{L}_{x}\left(\hat{G}_{n_{i(1)}(1)}^{i\left(\hat{C}^{i}\right.}\right)$ contains a $\left(1+2 \delta^{\prime}\right)^{r-2}$-scaled neighbourhood of $\mathcal{L}_{x}\left(\hat{G}_{n_{i(r)}}^{i(r)}\right)$. Since $\mathcal{L}_{x}(J)=$ $G_{0} \subset \mathcal{L}_{x}\left(\hat{G}_{n_{i(r)}}^{i(r)}\right)$ and $\mathcal{L}_{x}\left(\hat{G}_{n_{i(1)}^{i(1)}}^{i(j)} \subset \mathcal{L}_{x}(I)\right.$. Hence $\mathcal{L}_{x}(I)$ is a $\left(1+2 \delta^{\prime}\right)^{r-2}$-scaled neighbourhood of $\mathcal{L}_{x}(J)$. This completes the argument if $\kappa$ and hence $r$ is large.

This concludes the proof of Lemma 3.12.

Lemma 3.15 (cf. [KSvS], Lemma 9.6). For any $C>0$ and $\delta>0$ there exists $C^{\prime}>0$ such that the following holds. Let I be a nice interval and $J$ a pullback of I with $I \supset\left(1+2 C^{\prime}\right) J$ and $c_{0} \in J$. Assume that either

- $c_{0}$ is a critical point of even order or

- I is $\delta$-nice.

Then for any $x \in \omega\left(c_{0}\right)$, we have that $(1+2 C) \mathcal{L}_{x}(J) \subset \mathcal{L}_{x}(I)$.

Remark. The proof of this lemma in the case when $c_{0}$ is a critical point of odd order is significantly more difficult than when it has even order. The reason for this is that if $I \supset K \supset J \ni c_{0}$ are intervals so that $J$ deep-inside $I$, then unless $I, K$ and $J$ are symmetric with respect to $c_{0}$ it is quite possible that $K$ not well-inside $I$ and also that $J$ is not well-inside $K$. A similar issue arises in the proof of Lemma 3.16.

Proof. Assume that $x \in \omega\left(c_{0}\right)$. Observe that since $f$ is persistently recurrent on $\omega\left(c_{0}\right)$ and $c_{0} \in J$, there exists $k>0$ such that $f^{k}(x) \in J$. Let $I^{n}=\mathcal{L}_{c_{0}}^{n}(I)$ and define $m(0)=0$ and $m(1)<m(2)<\cdots$ as the positive integers such that $R_{I^{m(i)-1}}\left(c_{0}\right) \notin I^{m(i)}$. Let $k_{0}$ be maximal such that $J \subset I^{m\left(k_{0}\right)}$. Notice that $J$ could be equal to $I^{m\left(k_{0}\right)}$.

Claim 1. We can assume that $k_{0}$ is uniformly bounded.

Proof of Claim 1. By Lemma 3.8, for any $i$ with $1<i \leq k_{0}-1$ the interval $I^{m(i)}$ contains a definite neighbourhood of $I^{m(i)+1}$. By the Theorem 3.2 (1), we know that $\mathcal{L}_{x}\left(I^{m(i)}\right)$ contains a definite neighbourhood of $\mathcal{L}_{x}\left(I^{m(i)+1}\right)$. As $\mathcal{L}_{x}(J) \subset$ $\mathcal{L}_{x}\left(\mathcal{L}_{c_{0}}\left(I^{m\left(k_{0}-1\right)+1}\right)\right)$ and $\mathcal{L}_{x}\left(I^{m(1)}\right) \subset \mathcal{L}_{x}\left(I^{0}\right)$, the lemma follows if $k_{0}$ is sufficiently large.

Case 1. Assume that $c_{0}$ is even. Suppose first that $k_{0}=0$. If additionally, $m(1)<4$ or if $I^{j} \subset J$ for $j \in\{1,2,3\}$ we get that $J \supset I^{4}=\mathcal{L}_{c_{0}}^{4}(I)$, and the result follows from Lemma 3.12. So we assume $J \subset I^{2}$ and $m(1) \geq 4$. If $\left|I^{j}\right| /\left|I^{j+1}\right|>C_{1} / 3$ is large for $j \in\{0,1\}$ and some $C_{1}>1$, then $\left(1+2 C_{1}\right) I^{j+1} \subset I^{j}$. From Lemma 3.12, and making $C_{1}$ larger if necessary, we know that $(1+2 C) \mathcal{L}_{x}\left(I^{j+1}\right) \subset \mathcal{L}_{x}\left(I^{j}\right)$. Since $\mathcal{L}_{x}(J) \subset \mathcal{L}_{x}\left(I^{j+1}\right) \subset \mathcal{L}_{x}\left(I^{j}\right) \subset \mathcal{L}_{x}\left(I^{0}\right)$, the result follows. On the other hand if $\left|I^{0}\right| /\left|I^{2}\right|$ is not large, Lemma 3.10 implies that $\left|I^{m(1)-1}\right|$ is comparable to $\left|I^{0}\right|$, so $J$ is deep-inside $I^{m(1)-1}$ and the lemma follows from Lemma 3.12.

Let us now assume that $k_{0}>0$. Then, there are two possibilities: either

- $\left|I^{m(j)}\right| /\left|I^{m(j+1)}\right|$ is close to one for all $0 \leq j \leq k_{0}-1$ or

- there exist $j_{0}$ with $0 \leq j_{0} \leq k_{0}-1$ so that $\left|I^{\left.\overline{m(} j_{0}\right)}\right| /\left|I^{m\left(j_{0}+1\right)}\right|$ is large.

In either case the result follows from the case $k_{0}=0$ applied to intervals $I^{\prime}$ and $J^{\prime}$ with $J \subset J^{\prime} \subset I^{\prime} \subset I^{0}$ as follows. In the first case, we consider $I^{\prime}=I^{m\left(k_{0}-1\right)}$ and $J^{\prime}=J$, and in the second case we consider $I^{\prime}=I^{m\left(j_{0}\right)}$ and $J^{\prime}=I^{m\left(j_{0}+1\right)}$. This concludes the proof when $c_{0}$ is even.

Case 2. Assume $c_{0}$ is odd. Then all critical points in $\omega\left(c_{0}\right)$ are odd. Let $m^{\prime} \in \mathbb{N}$ be so that $I^{m^{\prime}} \subset J \subset I^{m^{\prime}-1}$. If $m^{\prime}<6$ the result follows from Lemma 3.12, so from now on we will assume $J \subset I^{5}$. Since $I$ is $\delta$-nice by Corollary 3.4 there exits $\delta^{\prime}>0$ so that $I^{1}$ and $I^{2}$ are $\delta^{\prime}$-nice. 
Claim 2. We can assume that $\left|I^{1}\right|$ is comparable to $\left|I^{3}\right|$ and that there exists a large constant $C_{2}=C_{2}(C, \delta)>1$ such that $\left(1+2 C_{2}\right) J \subset I^{2}$.

Proof of Claim 2. We can assume that $\left|I^{2}\right|$ is comparable to $\left|I^{1}\right|$. Indeed, if $\left|I^{2}\right|$ is very small compared $\left|I^{1}\right|$, then because $I^{1}$ is well-inside $I^{0}$ we get that $I^{2}$ is deep-inside $I^{0}$ and the lemma follows from Lemma 3.12. Similarly, we can assume that $\left|I^{3}\right|$ is comparable to $\left|I^{2}\right|$. Therefore, from now on, we can and will assume that $\left|I^{3}\right|$ is comparable to $\left|I^{1}\right|$. If $|J|$ is comparable to $\left|I^{1}\right|$, then, since $J$ is deep-inside of $I^{0}$, we have that $I^{1}$ is deep-inside $I^{0}$, and the result follows from Lemma 3.12. So we can assume $|J|$ is small compared to $\left|I^{1}\right|$ and indeed also to $\left|I^{3}\right|$. Furthermore, since $J \subset I^{3},\left(1+2 \delta^{\prime}\right) I^{3} \subset I^{2}$ and $|J|$ is small compared to $\left|I^{3}\right|$ we get that $J$ is deep-inside $I^{2} . \checkmark$

Let us first study the cases when $k_{0}=0$ or when $k_{0}=1$ and $I^{m(1)+1} \subset J \subset I^{m(1)}$. Observe that since $m^{\prime}>6$ we must have that $m(1)>5$. Let $r$ be the return time of $c_{0}$ into $I^{0}$ and let $f^{r}: I^{1} \rightarrow I^{0}$ be the return map. Since $c_{0}$ is odd, $f^{r} \mid I^{1}$ is a homeomorphism. Let $L^{\prime}=\left(1+\delta^{\prime}\right) I^{1}$ and $L=\operatorname{Comp}_{c_{0}} f^{-r}(L) \supset I^{2}$. By the choice of $\delta^{\prime}$ we know that $L \subset I^{0}$ and Theorem 3.2(2) implies that $f^{r}: L \rightarrow L^{\prime}$ is a (finite) composition of polynomials and maps with bounded distortion. From Claim 2, we can assume that $\left|I^{1}\right|$ is comparable to $\left|I^{2}\right|$, so Lemma 3.10(2) implies that $\left|D f^{r}\right|$ is bounded from above on $I^{2}$ and that $\left|c-f^{r}(c)\right| /\left|I_{2}\right|$ is bounded from below for any $c \in \operatorname{Crit}\left(\left.f^{r}\right|_{I^{2}}\right)$. Even more, if we let $g=\left(\left.f^{r}\right|_{I^{1}}\right)^{-1}$ we get that $\left|c_{0}-g\left(c_{0}\right)\right| /\left|I_{2}\right|$ is bounded from below. The map $\left.f^{r}\right|_{I^{1}}$ is monotone so by definition of $I^{m(1)}$ the points $c_{0}, g\left(c_{0}\right)$ and $f^{r}\left(c_{0}\right)$ are contained in $I^{m(1)-1}$, and $c_{0}$ lies between $g\left(c_{0}\right)$ and $f^{r}\left(c_{0}\right)$. Since $\left|c_{0}-g\left(c_{0}\right)\right| /\left|I_{2}\right|$ and $\left|c_{0}-f^{r}\left(c_{0}\right)\right| /\left|I_{2}\right|$ are bounded from below, $\left\{g\left(c_{0}\right), c_{0}, f^{r}\left(c_{0}\right)\right\} \subset I^{m(1)-1}, c_{0} \in J$, and $J$ is deep-inside $I^{2}$, we have that $J$ is deep-inside $I^{m(1)-1}$. We are assuming $J \supset I^{m(1)+1}$, so in this case the lemma follows from Lemma 3.12.

Next, let us assume that $I^{m(2)} \subset J \subset I^{m(1)+1}$. If $\left|I^{m(1)+1}\right|$ is comparable to $|J|$, then $I^{m(1)+1}$ is deep-inside $I^{2}$. Applying the case $k_{0}=1$ to the intervals $I^{\prime}=I^{2}$ and $J^{\prime}=I^{m(1)+1}$, we get that $(1+2 C) \mathcal{L}_{x}\left(J^{\prime}\right) \subset \mathcal{L}_{x}\left(I^{2}\right)$. Since $\mathcal{L}_{x}(J) \subset \mathcal{L}_{x}\left(J^{\prime}\right)$ we are done in this case. Assume that $|J|$ is small compared to $\left|I^{m(1)+1}\right|$. Let $k>0$ be minimal so that $f^{k}\left(c_{0}\right) \subset I^{0} \backslash I^{1}$ and let $K=\mathcal{L}_{f^{k}\left(c_{0}\right)}(I)$. Since $J \subset I^{m(1)+1}$ and $k$ is minimal we have that $c_{0}$ visits $K$ before returning to $I^{m(1)}$. Since $I$ is $\delta$-nice and $K$ is a return domain to $I^{0}$, we can apply Theorem 3.2 (1) to find $\delta^{\prime}>0$ so that $K^{\prime}=\left(1+2 \delta^{\prime}\right) \mathcal{L}_{f^{k}\left(c_{0}\right)}\left(I^{m(1)}\right) \subset K$. Let $\left\{G_{i}^{\prime}\right\}_{i=0}^{k}$ be the chain given by $K=G_{k}^{\prime}$ and $G_{0}^{\prime}=\mathcal{L}_{c_{0}}(K)$ and $\left\{G_{i}\right\}_{i=0}^{k}$ be the chain given by $K^{\prime}=G_{k}$ and $G_{0}=\mathcal{L}_{c_{0}}\left(K^{\prime}\right)$. Since $k$ is the first entry time of $c_{0}$ to $K$ each of these chains has order bounded by $b$. By Lemma 3.3 there exists $\delta_{2}>0$ so that $\left(1+2 \delta_{2}\right) \mathcal{L}_{c_{0}}\left(K^{\prime}\right) \subset \mathcal{L}_{c_{0}}(K) \subset I^{m(1)}$. Since $I^{m(1)+1} \subset \mathcal{L}_{c_{0}}\left(K^{\prime}\right)$ and $J$ is small compared to $I^{m(1)+1}$, we have that $J$ is deep-inside $I^{m(1)}$. Since $I^{m(2)} \subset J \subset I^{m(1)+1}$, taking $I^{\prime}=I^{m(1)}$ and $J^{\prime}=J$ the result follows from the case $k_{0}=0$.

Finally, let us now consider the case when $k_{0} \geq 2$. Recall, $k_{0}$ is maximal such that $J \subset I^{m\left(k_{0}\right)}$. If $J$ is deep-inside $I^{m\left(k_{0}\right)}$, the result follows from the case $k_{0}=0$, so assume this is not the case. There are two possibilities, either

(1) $J$ is small compared to $I^{m\left(k_{0}\right)}$ and $d\left(\partial I^{m\left(k_{0}\right)}, J\right) /|J|$ is bounded from above or

(2) $|J|$ is comparable to $\left|I^{m\left(k_{0}\right)}\right|$.

Assume that (1) holds. From the definition of the intervals $I^{m(i)}$ and the fact that $k_{0} \geq$ 2, since $I^{m\left(k_{0}\right)} \subset \mathcal{L}_{c_{0}}\left(I^{m\left(k_{0}-1\right)}\right)$, we can apply Lemma 3.8 to find $\delta_{3}>0$ so that $\left(1+2 \delta_{3}\right) I^{m\left(k_{0}\right)} \subset I^{m\left(k_{0}-1\right)}$. This implies that $J$ is deep-inside of $I^{m\left(k_{0}-1\right)}$. Taking $I^{\prime}=I^{m\left(k_{0}-1\right)}$ and $J^{\prime}=J$, the result follows either from the case $I^{m(1)+1} \subset J \subset I^{m(1)}$ or from the case $I^{m(2)} \subset J \subset I^{m(1)+1}$. So we can assume that (2) holds; that is, $|J|$ 
is comparable to $\left|I^{m\left(k_{0}\right)}\right|$. Then $I^{m\left(k_{0}\right)}$ is small compared to $I^{0}$. Since, by Claim $1, k_{0}$ is bounded from above, we must have that one of the ratios $\left|I^{m(j)}\right| /\left|I^{m(j+1)}\right|$ is big for some $j \in\left\{0, \ldots k_{0}-1\right\}$. Let $j_{0}$ be maximal with this property. If $j_{0}=0$, then $\left|I^{m(1)}\right|$ is comparable to $|J|$, and $\left|I^{m(j)}\right|$ is comparable to $\left|I^{m(j+1)}\right|$, for any other $j \in 1, \ldots k_{0}-1$. This gives us that $\left|I^{m(1)}\right|$ is comparable to $|J|$, so that $J^{\prime}=I^{m(1)}$ must be deep-inside $I^{\prime}=I^{0}$, and we argue as in the case when $k_{0}=0$. Suppose $j_{0}=1$. Since $I$ is $\delta$ nice, $I^{m(1)+1}$ is well-inside $I^{m(1)}$, so $I^{m(2)}$ is deep-inside $I^{m(1)}$. Setting $I^{\prime}=I^{m(1)}$ and $J^{\prime}=I^{m(2)}$. If $j_{0}>1$, then by Lemma 3.8 and the definition of the intervals $I^{m(j)}$, we have that $I^{m\left(j_{0}+1\right)}$ is well-inside $I^{m\left(j_{0}\right)}$, so $I^{m\left(j_{0}+1\right)}$ is deep-inside $I^{m\left(j_{0}-1\right)}$. We take $I^{\prime}=I^{m\left(j_{0}+1\right)}$ and $J^{\prime}=I^{m\left(j_{0}-1\right)}$. In either of these last two cases, we conclude the proof as we did when (1) holds.

Lemma 3.16 (cf. [KSvS], Lemma 9.7). For each $\delta, \rho>0$ and each integer $N \geq 0$, there exist $\rho^{\prime}, \delta^{\prime}>0$ so that the following holds. Let $I$ and $J$ be nice intervals with $I \supset(1+2 \rho) J \supset J \ni c_{0}$ and $J$ is a pullback of I with order bounded by N. Let $\left\{G_{j}\right\}_{j=0}^{s}$ be the chain associated to the pullback and assume one of the following conditions holds:

- $c_{0}$ is a critical point of even order.

- $c_{0}$ is a critical point of odd order, $I$ is $\delta$-nice and $G_{i} \cap J=\emptyset$ for $i=1,2, \ldots, s-1$.

Then $J$ is $\rho^{\prime}$-nice, where $\rho^{\prime}$ is defined by a function $\rho^{\prime}=\rho^{\prime}(\rho)>0$ depending on $N$ and $\delta$ such that $\rho^{\prime} \rightarrow \infty$ as $\rho \rightarrow \infty$.

Proof. Let $\left\{G_{j}\right\}_{j=0}^{s}$ be the chain with $G_{s}=I$ and $G_{0}=J$. Let us first assume that none of the intervals from the chain $\left\{G_{i}\right\}_{i=1}^{s-1}$ intersect $J$. Given $x \in J \cap \omega\left(c_{0}\right)$ so that $r$ is the return time of $x$ to $J$ we must have that $r \geq s$. Let $y=f^{s}(x)$. By the previous lemma we know that there exists $\rho_{1}>0, \rho_{1} \rightarrow \infty$ as $\rho \rightarrow \infty$, so that $\left(1+2 \rho_{1}\right) \mathcal{L}_{y}(J) \subset$ $\mathcal{L}_{y}(I)$. Lemma 3.3 implies the existence of $\rho^{\prime}>0, \rho^{\prime} \rightarrow \infty$ as $\rho_{1} \rightarrow \infty$, so that $\left(1+2 \rho^{\prime}\right) \operatorname{Comp}_{x} f^{-s}\left(\mathcal{L}_{y}(J)\right) \subset J$, which means that $\left(1+2 \rho^{\prime}\right) \mathcal{L}_{x}(J) \subset J$.

Assume there exists an element of $\left\{G_{i}\right\}_{i=1}^{s-1}$ that intersects $J$. Let $s^{\prime}<s$ be maximal so that $G_{s^{\prime}} \cap J \neq \emptyset$. By assumption we must have that $c_{0}$ is a critical point of even order, so by symmetry we have that for $\rho_{2}=\rho / 2$ either $\left(1+2 \rho_{2}\right) G_{s^{\prime}} \subset I$ or $\left(1+2 \rho_{2}\right) J \subset G_{s^{\prime}}$. Assume $\left(1+2 \rho_{2}\right) G_{s^{\prime}} \subset I$. The maximality of $s^{\prime}$ implies that none of the elements of the chain $\left\{G_{i}\right\}_{i=s^{\prime}+1}^{s-1}$ intersect $J$. So we can apply the previous argument to find $\rho_{3}>0$ such that $G_{s^{\prime}}$ is $\rho_{3}-$ nice. This means that $\left(1+2 \rho_{3}\right) J \subset G_{s^{\prime}}$. If we are in this case or in the case that $\left(1+2 \rho_{2}\right) J \subset G_{s^{\prime}}$ the proof follows by applying the previous argument at most $N-1$ times, since $J$ is a pullback of $G_{S^{\prime}}$ of order less than $N$.

Remark. the first part of the proof also shows the following. Assume that $c_{0}$ is a critical point of odd order and that there exists exactly one $s^{\prime} \in\{1,2, \ldots, s-1\}$ such that $G_{S^{\prime}} \cap J \neq \varnothing$. Then, if $x \in J$ is such that $f^{k}(x) \notin J$, where $k>0$ is minimal so that $f^{k}(x) \in G_{s^{\prime}}$, we have that $\left(1+2 \rho^{\prime}\right) \mathcal{L}_{x}(J) \subset J$.

Before we continue, recall that $\Gamma(I)$ is the smallest successor of $I$; so $\Gamma(I)$ is a pullback of $I$ of order bounded by $2 b-1$.

Lemma 3.17 (cf. [KSvS], Lemma 9.8). There exists a universal constant $\delta>0$ such that if $I$ is a nice interval containing $c_{0}$ and both $I$ and $\mathcal{C}_{c_{0}}(I)$ are non-terminating, then $(1+2 \delta) \Gamma^{2}(I) \subset I$. 
Proof. Let $I$ be a nice interval containing a point $c_{0}$. If $U$ is a nice interval in $I$ that is disjoint from $\mathcal{L}_{c_{0}}(I)$, then any pullback of $U$ that contains $c_{0}$ is contained in $\mathcal{C}_{c_{0}}(I)$, see [vSV, Lemma 15].

Since $I$ is non-terminating, $\omega\left(c_{0}\right)$ intersects a non-central domain of the return map to $I$ and therefore, by the above statement, $\Gamma(I) \subset \mathcal{C}_{c_{0}}(I)$. Since $\mathcal{C}_{c_{0}}(I)$ is non-terminating, $\omega\left(c_{0}\right)$ intersects a non-central domain of the return map to $\mathcal{C}_{c_{0}}(I)$, so again we have that any pullback of this domain is contained in $\mathcal{C}_{c_{0}}^{2}(I)$. Hence $\Gamma^{2}(I) \subset \mathcal{C}_{c_{0}}^{2}(I)$. By Lemma 3.8, it follows that $(1+2 \delta) \Gamma^{2}(I) \subset \mathcal{C}_{c_{0}}(I)$.

Lemma 3.18. Suppose that $I \ni c_{0}$ is a nice interval and additionally if $c_{0}$ is odd, that I is $\delta$-nice for some $\delta>0$. Then, there exists a constant $\hat{\delta}>0$, that is universal if $c_{0}$ is even, and $\hat{\delta}=\hat{\delta}(\delta)$ if $c_{0}$ is odd, so that if $\Gamma^{2}(I)$ is non-terminating, then $\Gamma^{3}(I)$ is $\hat{\delta}$-nice. Moreover, for each $\rho>0$ there exists $\rho^{\prime}>0$ with $\rho^{\prime} \rightarrow \infty$ as $\rho \rightarrow \infty$, so that if $(1+2 \rho) \Gamma^{2}(I) \subset I$, then $\left(1+2 \rho^{\prime}\right) \Gamma^{3}(I) \subset \Gamma^{2}(I)$ and $\Gamma^{3}(I)$ is $\rho^{\prime}$-nice.

Proof. Assume $c_{0}$ is even. Since $\Gamma^{2}(I)$ is non-terminating, both $\Gamma(I)$ and $C_{c_{0}}(I)$ are non-terminating. So the result follows from Lemmas 3.17 and 3.16. If $c_{0}$ is odd then we argue as follows. Let $J$ be a successor of $I$, and let $\left\{J_{j}\right\}_{j=0}^{k}$ be the chain with $J_{0}=J$ and $J_{k}=I$. Then, it is not hard to see that the only elements of the chain containing the point $c_{0}$ are $G_{0}$ and $G_{k}$. This means that is we consider the chain $\left\{G_{j}\right\}_{j=0}^{s}$ with $G_{0}=\Gamma^{2}(I)$ and $G_{s}=I$ there is only one $s^{\prime}, 0<s^{\prime}<s$, with $G_{s^{\prime}} \cap \Gamma^{2}(I) \neq \emptyset$. Using the remark at the end of the proof of Lemma 3.16 it follows that all but one of the domains of the first return map to $\Gamma^{2}(I)$ is $\rho$-well-inside $\Gamma^{2}(I)$. Since $\Gamma^{2}(I)$ is non-terminating, the orbit of $\Gamma^{3}(I)$ passes through a non-central return domain of $\Gamma^{2}(I)$. It follows that $(1+2 \rho) \Gamma^{3}(I) \subset \Gamma^{2}(I)$. From Lemma 3.16 it follows that $\Gamma^{3}(I)$ is $\rho^{\prime}(\rho)$-nice.

Lemma 3.19 (cf. [KSvS], Lemma 9.9). For any $\rho>0$ there exists $\rho^{\prime}>0$, with $\rho^{\prime} \rightarrow \infty$ as $\rho \rightarrow \infty$, such that if $I$ is a $\rho$-nice interval containing $c_{0}$, then $\left(1+2 \rho^{\prime}\right) \mathcal{A}(I) \backslash \mathcal{A}(I)$ is disjoint from $\omega\left(c_{0}\right)$ and contained in $\mathcal{B}(I)$. Moreover, if $c_{0}$ is of even order, then $\mathcal{B}(I) \backslash\left(1+2 \rho^{\prime}\right)^{-1} \mathcal{B}(I)$ is disjoint from $\omega\left(c_{0}\right)$. If $c_{0}$ is of odd order, then for each $v>0$ and each $\rho>0$ there exists $\rho^{\prime \prime}>0$, so that if $|\mathcal{A}(I)| /|\mathcal{B}(I)| \geq v$, then $\mathcal{B}(I) \backslash\left(1+2 \rho^{\prime \prime}\right)^{-1} \mathcal{B}(I)$ is disjoint from $\omega\left(c_{0}\right)$.

Proof. By definition we know that $\mathcal{B}(I)$ and $\mathcal{A}(I)$ are both pullbacks of $I$ of bounded order (where the bound depends only on $b$ ). Since $I$ is $\rho$-nice we can apply Lemma 3.3 to find $\rho^{\prime}>0$ so that $\left(1+2 \rho^{\prime}\right) \mathcal{A}(I) \subset \mathcal{B}(I)$. By definition $\mathcal{B}(I) \backslash \mathcal{A}(I)$ is disjoint from $\omega\left(c_{0}\right)$. So $\left(1+2 \rho^{\prime}\right) \mathcal{A}(I) \backslash \mathcal{A}(I)$ is disjoint from $\omega\left(c_{0}\right)$. If $|\mathcal{A}(I)|$ is comparable to $|\mathcal{B}(I)|$ then the above implies the existence of $\rho^{\prime \prime}>0$ so that $\mathcal{B}(I) \backslash\left(1+2 \rho^{\prime \prime}\right)^{-1} \mathcal{B}(I) \subset$ $\mathcal{B}(I) \backslash \mathcal{A}(I)$ and the result follows. So the only case left to consider is when $c_{0}$ is even and $|\mathcal{A}(I)|$ is small compared to $|\mathcal{B}(I)|$. Since $c_{0}$ is even, this implies $\mathcal{A}(I)$ is deep-inside $\mathcal{B}(I)$, so the components of $\mathcal{B}(I) \backslash \mathcal{A}(I)$ have size comparable to $\mathcal{B}(I)$ and the result follows.

3.5. Geometry of periodic intervals. In this subsection, we study the geometry of terminating intervals. Before we begin, let us refer the reader to Sect. 2 for the relevant notation. Recall that given $I_{i}$, a terminating interval from generalized enhanced nest, $\mathcal{R}\left(I_{i-1}\right)$ is Feigenbaum if and only if it is a periodic interval, necessarily of period two.

Proposition 3.20. For each $v>0$ there exists $\rho>0$ such that the following holds. Assume that $I_{i-1}$ is a terminating interval from the enhanced nest. 
(1) If $\mathcal{R}\left(I_{i-1}\right)$ is terminating and not periodic, then $\mathcal{R}\left(I_{i-1}\right)$ is $\rho$-free.

(2) If $\mathcal{R}\left(I_{i-1}\right)$ and $\mathcal{R}^{2}\left(I_{i-1}\right)$ are Feigenbaum, then $\mathcal{R}\left(I_{i-1}\right)$ is $\rho$-free.

(3) If $\mathcal{R}\left(I_{i-1}\right)$ is non-terminating and $\left|\mathcal{R}\left(I_{i-1}\right)\right| /\left|I_{i+1}\right|<v$, then $\mathcal{R}\left(I_{i-1}\right)$ is $\rho$-free.

(4) If $\mathcal{R}\left(I_{i-1}\right)$ is Feigenbaum, $\mathcal{R}\left(I_{i}\right)$ is not periodic and $\left|I_{i}\right| /\left|I_{i+2}\right|<v$, then $\mathcal{R}\left(I_{i-1}\right)$ is $\rho$-free.

Proof. Let $r>0$ be minimal so that $f^{r}\left(c_{0}\right) \in I_{i-1}$. Since $I_{i-1}$ is terminating, $I_{i-1}^{\infty}=\cap_{n \geq 0} I_{i-1}^{n}, f^{r}\left(c_{0}\right) \in I_{i-1}^{\infty}$, and $f^{r} \mid I_{i-1}^{\infty}$ maps $I_{i-1}^{\infty}$ into itself. Thus, we have that $\mathcal{L}_{c_{0}}^{n}\left(I_{i-1}^{\infty}\right)=I_{i-1}^{\infty}$, for all $n \in \mathbb{N}$, is an infinite central cascade. Since $I_{i-1}$ is terminating, we know that $I_{i}=\mathcal{L}_{c_{0}}\left(\mathcal{R}\left(I_{i-1}\right)\right)$.

Apply Lemma 3.10 with $\delta=1$, fixed, to obtain constants $C, \kappa>0$ such that:

(a) If $c \in$ Crit $\left.f^{r}\right|_{I_{i-1}^{\infty}}$ we have $\left|f^{r}(c)-c\right| \geq \kappa\left|I_{i-1}^{\infty}\right|$;

(b) $\left|D f^{r}(x)\right| \leq C$ for all $x \in I_{i-1}^{\infty}$;

(c) Given $\left.c \in \operatorname{Crit} f^{r}\right|_{I_{i-1}^{\infty}}$ and $p=f^{r}(p)$ both in $I_{i-1}^{\infty}$, we have $|p-c| \geq \kappa\left|I_{i-1}^{\infty}\right|$,

where the third statement follows from the first two. We will refer to these estimates throughout the proof.

Let $V_{0}$ be the closure of the landing domain to $\mathcal{R}\left(I_{i-1}\right)$ containing $\alpha$ in its boundary and $\hat{V}_{0}=\operatorname{Comp}_{\alpha} f^{-r}\left(V_{0}\right) \cup V_{0}$.

Proof of 1. Since $\mathcal{R}\left(I_{i-1}\right)$ is terminating and not Feigenbaum, $f^{r}\left(V_{0}\right)=\mathcal{R}\left(I_{i-1}\right)$, and $V_{0}$ is disjoint from $\omega\left(c_{0}\right)$. This implies that $\hat{V}_{0}$ is disjoint from $\omega\left(c_{0}\right)$. By (a) and (b) there exists $\rho>0$ such that $\left|V_{0}\right|>\rho\left|\mathcal{R}\left(I_{i-1}\right)\right|$, and $\left|\hat{V}_{0}\right|$ is comparable to $\left|I_{i-1}\right|$. Since $c_{0}$ is even, the result follows by symmetry.

Proof of 2. Let us assume $\mathcal{R}\left(I_{i-1}\right)$ is not $\rho$-free. Then there exist $m \in \mathbb{N}$ such that $f^{2 r m}\left(c_{0}\right)$ is close to the boundary of $\mathcal{R}\left(I_{i-1}\right)$. Regardless of whether $f^{2 r m}\left(c_{0}\right)$ is close to $\alpha$ or $\tau(\alpha)$, we must have that $f^{2 r(m+1)}\left(c_{0}\right)$ is close to $\alpha$, which is a contradiction. To see this, observe that if $R^{2}\left(I_{i-1}\right)$ is Feigenbaum, either $f^{2 r m}\left(c_{0}\right)$ or $f^{2 r(m+1)}\left(c_{0}\right)$ must be contained in $R^{2}\left(I_{i-1}\right)$, which is well-inside $\mathcal{R}\left(I_{i-1}\right)$ by (b) and (c). So it cannot happen that both $f^{2 r m}\left(c_{0}\right)$ and $f^{2 r(m+1)}\left(c_{0}\right)$ are close to the boundary of $\mathcal{R}\left(I_{i-1}\right)$.

Proof of 3. Assume $\mathcal{R}\left(I_{i-1}\right)$ is non-terminating and $\left|\mathcal{R}\left(I_{i-1}\right)\right| /\left|I_{i+1}\right|<v$. The return map to $I_{i}$ restricted to $V_{0}$ is equal to $f^{2 r}$. If $V_{0}$ does not intersect $\omega\left(c_{0}\right)$, we are done, so let us assume $V_{0}$ intersects $\omega\left(c_{0}\right)$. Let $V_{j+1}$ be the pullback of $V_{j}$ under $f^{2 r}$ containing $\alpha$ for $j \in \mathbb{N}$. Observe that (b) implies that $\left|V_{1}\right|$ is comparable $\left|\mathcal{R}\left(I_{i-1}\right)\right|$, so if $\omega\left(c_{0}\right)$ does not intersect $V_{1}$ the proposition follows. Let us assume $V_{1}$ contains some point in $\omega\left(c_{0}\right)$. We will show that $\left.f^{2 r}\right|_{V_{1}}$ is monotone. Let $c^{\prime}$ be the turning point of $\left.f^{2 r}\right|_{V_{0}}$ closest to $\alpha$. By construction $c^{\prime}$ is an absolute maximum (or minimum if $\tau(\alpha)<\alpha$ ). If $f^{2 r}\left(c^{\prime}\right)$ is not in $V_{0}$, then $\left.f^{2 r}\right|_{V_{1}}$ is monotone. If $f^{2 r}\left(c^{\prime}\right) \in V_{0}$, then $f^{2 r}\left(V_{0}\right) \subset V_{0}$, which contradicts the fact that $\omega\left(c_{0}\right)$ is minimal. Since $\alpha$ is an orientation reversing fixed point of $f^{r}$, we have that $\left.f^{2 r}\right|_{V_{1}}$ is an orientation preserving monotone map with at most $b$ critical points. Define $\hat{V}_{1}=\operatorname{Comp}_{\alpha} f^{-r}\left(V_{1}\right) \cup V_{1}$ and $\hat{V}_{j+1}=\operatorname{Comp}_{\alpha} f^{-r}\left(\hat{V}_{j}\right)$ for $j \in \mathbb{N}$. Let $c^{\prime \prime} \in \operatorname{Crit}\left(f^{2 r}\right) \cap V_{1}$ be the critical point closest to $\alpha$. If $c^{\prime \prime}$ is not defined, then let $c^{\prime \prime}=c^{\prime}$ and $n_{0}=1$. Otherwise, let $n_{0}$ be maximal such that $c^{\prime \prime} \in V_{n_{0}}$. Statement (c) implies that there exists $\kappa^{\prime}>0$ so that $\left|V_{n_{0}}\right|>\kappa^{\prime}\left|\mathcal{R}\left(I_{i-1}\right)\right|$ The proposition follows if $V_{n_{0}}$ does not intersect $\omega\left(c_{0}\right)$, so we will assume that $V_{n_{0}}$ intersects $\omega\left(c_{0}\right)$. Notice that for every $n>n_{0}$ the map $f^{\left(n_{0}-n\right) r}: \hat{V}_{n} \rightarrow \hat{V}_{n_{0}}$ is a diffeomorphism. Assume $V_{n_{0}+2}=[\alpha, y]$. Then by Lemma 3.7, we know that there exists $M>0$ so that $\left|y-f^{2 r}(y)\right|>M\left|\mathcal{R}\left(I_{i-1}\right)\right|$. Since the derivative of $f^{r}$ is bounded on $I_{i-1}^{\infty}$, we can find $\rho>0$ such that

$$
(1+2 \rho) \hat{V}_{n_{0}+2} \subset \hat{V}_{n_{0}+1} .
$$


By Theorem 3.2 (1) we know that there exists $\delta_{2}>0$ such that for any $n \in \mathbb{N}$ with $n>2$

$$
\left(1+2 \delta_{2}\right) \hat{V}_{n_{0}+n+1} \subset \hat{V}_{n_{0}+n} .
$$

The minimality of $\omega\left(c_{0}\right)$ implies that there exist $m=n_{0}+2+m^{\prime}$ maximal such that $\hat{V}_{m} \cap \omega\left(c_{0}\right) \neq \emptyset$. Let $k_{0} \in \mathbb{N}$ be minimal with the property that $f^{k_{0} r}\left(c_{0}\right) \in \hat{V}_{m}$. Define $k<k_{0}$ maximal such that $f^{k r}\left(c_{0}\right) \in \mathcal{R}\left(I_{i-1}\right)$. By the definition of $k$, we have that $f^{k_{0} r}\left(c_{0}\right)$ is the first entry of $f^{k r}\left(c_{0}\right)$ to both $\hat{V}_{m}$ and $\hat{V}_{1}$. For each $j \geq n_{0}+2$ let $V_{j}^{\prime}$ be the pullback of $\hat{V}_{j}$ along the orbit $\left\{f^{k_{0} r}\left(c_{0}\right), f^{k_{0} r-1}\left(c_{0}\right), \ldots, f^{k r}\left(c_{0}\right)\right\}$ containing $f^{k r}\left(c_{0}\right)$. Notice that the intervals $V_{j}^{\prime}$ are nice intervals. Applying Lemma 3.3 we can find $\delta_{3}>0$ such that $\left(1+2 \delta_{3}\right) V_{j+1}^{\prime} \subset V_{j}^{\prime}$. Using Theorem 3.2 (1) we can find $\delta_{4}>0$ such that $\left(1+2 \delta_{4}\right) \mathcal{L}_{c_{0}}\left(V_{j+1}^{\prime}\right) \subset \mathcal{L}_{c_{0}}\left(V_{j}^{\prime}\right)$. So

$$
\left(1+2 m^{\prime} \delta_{4}\right) \mathcal{L}_{c_{0}}\left(V_{m}^{\prime}\right) \subset \mathcal{L}_{c_{0}}\left(V_{1}^{\prime}\right) .
$$

From this we can conclude that given any $v>0$ there exists $m^{\prime} \in \mathbb{N}$ such that if $m=n_{0}+2+m^{\prime}$ and $V_{m} \cap \omega\left(c_{0}\right) \neq \emptyset$, then $\left|\mathcal{R}\left(I_{i-1}\right)\right| /\left|I_{i+1}\right|>v$. This is because $\left|I_{i-1}^{\infty}\right|$ is always comparable to $\left|\mathcal{R}\left(I_{i-1}\right)\right|$ and

$$
I_{i+1} \subset \Gamma^{2}\left(\mathcal{R}\left(I_{i-1}\right)\right) \subset \mathcal{L}_{c_{0}}\left(V_{m}\right) \subset \mathcal{L}_{c_{0}}\left(\hat{V}_{m}\right) \subset I_{i-1}^{\infty} .
$$

It remains to show that $\Gamma^{2}\left(\mathcal{R}\left(I_{i-1}\right)\right) \subset \mathcal{L}_{c_{0}}\left(V_{m}\right)$. To this end, let $x$ be $f^{k_{0}}\left(c_{0}\right)$ the image of $c_{0}$ under the first landing map of $c_{0}$ to $V_{m}$. Then $\mathcal{L}_{c_{0}} \mathcal{L}_{x} \Gamma\left(\mathcal{R}\left(I_{i-1}\right)\right)$ contains $\Gamma^{2} \mathcal{R}\left(I_{i-1}\right)$, since $\mathcal{L}_{c_{0}} \mathcal{L}_{x} \Gamma\left(\mathcal{R}\left(I_{i-1}\right)\right)$ is the pullback of $\Gamma\left(\mathcal{R}\left(I_{i-1}\right)\right)$ by two landing maps, and so it has to be bigger than the last child of $\Gamma\left(\mathcal{R}\left(I_{i-1}\right)\right)$. But now $\mathcal{L}_{c_{0}} \mathcal{L}_{x} \Gamma\left(\mathcal{R}\left(I_{i-1}\right)\right)$ is contained in $\mathcal{L}_{c_{0}}\left(V_{m}\right)$.

Proof of 4. Assume $\mathcal{R}\left(I_{i-1}\right)$ is Feigenbaum, $\mathcal{R}\left(I_{i}\right)$ is not Feigenbaum and $\left|I_{i}\right| /\left|I_{i+2}\right|$ $<v$. Since $\mathcal{R}\left(I_{i-1}\right)$ is Feigenbaum, we know that $R\left(I_{i-1}\right)=I_{i}$. Since $\mathcal{R}\left(I_{i-1}\right)$ is Feigenbaum, and the return time of $c_{0}$ to $I_{i-1}$ is $r$, we have that the return time of $c_{0}$ to $I_{i}$ is equal to $2 r$. Let $\alpha$ be the fixed point of $f^{r}$ contained in the boundary of $I_{i}$. By statement (c), the distance between $c_{0}$ and $\alpha$ is comparable to $\left|I_{i-1}^{\infty}\right|$, so $\left|I_{i}\right|$ and $\left|I_{i-1}^{\infty}\right|$ are comparable.

Let $\alpha^{\prime}$ be the orientation reversing fixed point of $f^{2 r}$ contained in the boundary of $\mathcal{R}\left(I_{i}\right)$. Let $W_{1}$ be the closure of the component of $I_{i} \backslash\left\{f^{-2 r}\left(\alpha^{\prime}\right)\right\}$ that contains $\alpha$ in its boundary. Let $\hat{W}_{1}$ be the interior of $W_{1} \cup \operatorname{Comp}_{\alpha} f^{-r}\left(W_{1}\right)$ and $\hat{W}_{j+1}=\operatorname{Comp}_{\alpha} f^{-r}\left(\hat{W}_{j}\right)$ for $j \in \mathbb{N}$. Observe that $\hat{W}_{j} \subset I_{i-1}^{\infty}$ for all $j \in \mathbb{N}$. Since $I_{i}$ is Feigenbaum, (a) and (b) imply that $\left|W_{2}\right|$ is comparable to $\left|I_{i}\right|$. If $\omega\left(c_{0}\right)$ does not intersect $W_{2}, I_{i}$ is $\delta$-free, so assume $\omega\left(c_{0}\right)$ intersects $W_{2}$. Observe that $\left.f^{2 r}\right|_{W_{1}}$ is monotone. Using the same argument as in the proof of 3 we can find $\delta_{2}>0$ and $n_{0} \in \mathbb{N}$ such that given any $j>3$

$$
\left(1+2 \delta_{2}\right) \hat{W}_{n_{0}+j+1} \subset \hat{W}_{n_{0}+j}
$$

If $I_{i}$ is not $\delta$-free there exists, $m^{\prime}$ big such that the orbit of $c_{0}$ enters $\hat{W}_{m}$, with $m=n_{0}+2+m^{\prime}$. Let $k \in \mathbb{N}$ be minimal so that $f^{r k}\left(c_{0}\right) \in \hat{W}_{m}$. Let $k_{0}<k$ be minimal so that $f^{r k_{0}}\left(c_{0}\right) \in I_{i}$. By Theorem 3.2 (1) there exists $\delta_{4}>0$ such that $\left(1+2 m^{\prime} \delta_{4}\right) \mathcal{L}_{c_{0}}\left(\mathcal{L}_{f^{r k}\left(c_{0}\right)}\left(\hat{W}_{m}\right)\right) \subset I_{i-1}^{\infty}$. So we have that $I_{i+2}$ is deep-inside $I_{i-1}^{\infty}$, since

$$
I_{i+2} \subset \Gamma\left(\mathcal{R}\left(I_{i}\right)\right) \subset \mathcal{L}_{c_{0}}\left(\mathcal{L}_{f}^{r k_{0}\left(c_{0}\right)}\left(\mathcal{R}\left(I_{i}\right)\right)\right) \subset \mathcal{L}_{c_{0}}\left(\mathcal{L}_{f^{r k_{0}\left(c_{0}\right)}}\left(\hat{W}_{m}\right)\right) .
$$

We know that $\left|I_{i}\right|$ and $\left|I_{i-1}^{\infty}\right|$ are comparable, so $I_{i+2}$ being deep-inside $I_{i-1}^{\infty}$ contradicts $\left|I_{i}\right| /\left|I_{i+2}\right|<v$. 
Corollary 3.21. There exists $\delta>0$ and for each $\rho>0$ there exists $\rho^{\prime}>0$ such that the following holds. Let $I_{i-1}$ be a terminating interval from the enhanced nest.

- Assume $\mathcal{R}\left(I_{i-1}\right)$ is non-periodic. Let $V_{0}$ be the component of $f^{-2 r}\left(\mathcal{R}\left(I_{i-1}\right)\right)$ that contains $\alpha$ in its boundary and $W_{0}$ be the component of $f^{-2 r}\left(\mathcal{R}\left(I_{i-1}\right)\right)$ that contains $\tau(\alpha)$ in its boundary. If $x \in \mathcal{R}\left(I_{i-1}\right)$ and $\mathcal{L}_{x}\left(\mathcal{R}\left(I_{i-1}\right)\right)$ is different from $V_{0}$ and $W_{0}$, then $\mathcal{L}_{x}\left(\mathcal{R}\left(I_{i-1}\right)\right)$ is $\delta$-nice.

- If $\mathcal{R}\left(I_{i-1}\right)$ is $\rho$-free, then $\mathcal{L}_{x}\left(\mathcal{R}\left(I_{i-1}\right)\right)$ is $\rho^{\prime}$-free.

Proof. Let $V_{1}$ be the component of $f^{-4 r}\left(\mathcal{R}\left(I_{i-1}\right)\right)$ that contains $\alpha$ in its boundary and $W_{1}$ be the component of $f^{-4 r}\left(\mathcal{R}\left(I_{i-1}\right)\right)$ that contains $\tau(\alpha)$ in its boundary. From the proof of Proposition 3.20 we know that there exists $\rho_{1}>0$ such that $\left|V_{1}\right| \geq \rho_{1}\left|\mathcal{R}\left(I_{i-1}\right)\right|$. Since $W_{1}$ is the symmetric component, with respect to $c_{0}$, corresponding to $V_{1}$ the same bounds hold for $W_{1}$. The bounds on the size of $V_{1}$ and $W_{1}$ imply that $\mathcal{L}_{x}\left(\mathcal{R}\left(I_{i-1}\right)\right)$ is $\rho_{1}$ well-inside $\mathcal{R}\left(I_{i-1}\right)$ if $\overline{\mathcal{L}_{x}\left(\mathcal{R}\left(I_{i-1}\right)\right)} \cap\{\alpha, \tau(\alpha)\}=\emptyset$. This fact, along with Theorem 3.2 (1) imply the existence of $\delta>0$ such that $\mathcal{L}_{x}\left(\mathcal{R}\left(I_{i-1}\right)\right)$ is $\delta$-nice. If $\mathcal{R}\left(I_{i-1}\right)$ is $\rho$-free, we can apply Corollary 3.5 to get that $\mathcal{L}_{x}\left(\mathcal{R}\left(I_{i-1}\right)\right)$ is $\rho^{\prime}$-free, for some $\rho^{\prime}>0$.

Below we will make use of the sets $Y_{j}$ and $\tilde{\mathcal{Y}}_{\gamma}$ introduced on p. 21.

Lemma 3.22. There exists $\rho>0$ with the following property. Let $I_{i}$ be a terminating interval of the enhanced nest and $Y_{j}$ be a component of $I_{i}^{\infty} \backslash\left(f^{r} \mid I_{i}^{\infty}\right)^{-1}(\alpha)$. Given a point $x \in Y_{j}$ the following holds.

(1) If $Y_{j}$ does not intersect $\tilde{\mathcal{Y}}_{\alpha}$ and $-a<j<a$, then $Y_{j}$ is $\rho$-nice.

(2) Assume $j>-a$ and the first return time of $x$ to $Y_{j}$ equal to $f^{k r}$. If there exist $0<j_{0} \leq k$ such that $f^{r j_{0}}(x) \notin \tilde{\mathcal{Y}}_{\alpha}$, then $\mathcal{L}_{x}\left(Y_{j}\right)$ is $\rho$-nice.

(3) If $j=-a$ and the return time of $x$ to $Y_{j}$ is bigger than $f^{r}$, then $\mathcal{L}_{x}\left(Y_{j}\right)$ is $\rho$-nice.

Proof. Let $\tilde{Y}_{0}=Y_{0} \cap \tilde{\mathcal{Y}}_{\alpha}$ and $\tilde{Y}_{-1}=Y_{-1} \cap \tilde{\mathcal{Y}}_{\alpha}$. By Lemma 3.10, we can find $\kappa>0$ such than $\left|\tilde{Y}_{0}\right|,\left|\tilde{Y}_{-1}\right|>\kappa\left|I_{i}^{\infty}\right|$. It follows immediately that there exists $\rho^{\prime}>0$ such that the following holds. Given $Y_{j}$ with $j>-a$ and $J=\operatorname{Comp} f^{-r}\left(Y_{j}\right) \subset Y_{\ell}$ for some $\ell \in\{-a, \ldots, a\}$, if $J \cap \tilde{\mathcal{Y}}_{\alpha}=\emptyset$ then $\left(1+2 \rho^{\prime}\right) J \subset Y_{\ell}$. This implies (1). To prove (2), take $x \in Y_{j}$ with first return time to $Y_{j}$ equal to $f^{k r}$ and such that $f^{r j_{0}}(x) \notin \tilde{\mathcal{Y}}_{\alpha}$ for some $0<j_{0} \leq k$. Then, $\left(1+2 \rho^{\prime}\right) \mathcal{L}_{x}\left(Y_{j}\right) \subset Y_{j}$. By Theorem 3.2, we know that here exists $\rho>0$ such that $\mathcal{L}_{x}\left(Y_{j}\right)$ is $\rho$-nice. One can prove (3) in a similar way.

3.6. Proof of Theorem 3.1. We will prove Theorem 3.1 in two separate stages. First we will show parts (a) to (e).

Part (a). If $I_{n-1}$ is non-terminating, $I_{n}=\Gamma^{2}\left(\Gamma^{T-2}\left(\mathcal{B}\left(\mathcal{A}\left(I_{n-1}\right)\right)\right)\right)$, and by Lemma 3.18 we get that $I_{n}$ is $\rho_{1}$-nice for some $\rho_{1}>0$ which depends only on $\underline{b}$. If $I_{n-1}$ is terminating the result follows from Corollary 3.21 and the observation that $I_{n}$ being non-terminating implies that $I_{n}=\mathcal{L}_{x}\left(\mathcal{R}\left(I_{n-1}\right)\right)$ is different from $V_{0}$ and $W_{0}$. In conclusion, if $I_{n}$ is non-terminating we have that $I_{n}$ is $\rho_{1}$-nice.

Now assume $I_{n-1}$ is non-terminating. Let $v_{1} \in \mathbb{N}$ be so that $\mathcal{B A}\left(I_{n-1}\right)=$ $\operatorname{Comp}_{c_{0}} f^{-v_{1}}\left(\mathcal{A}\left(I_{n-1}\right)\right)$, and $\mathcal{A}^{2}\left(I_{n-1}\right)=\operatorname{Comp}_{c_{0}} f^{-v_{1}}\left(\mathcal{L}_{f^{v_{1}}\left(c_{0}\right)} \mathcal{A}\left(I_{n-1}\right)\right)$. Since $\mathcal{B}$ $\left(I_{n-1}\right) \backslash \mathcal{A}\left(I_{n-1}\right)$ is free from $\omega\left(c_{0}\right)$, we get that $\mathcal{B}^{2}\left(I_{n-1}\right)=\operatorname{Comp}_{c_{0}} f^{-v_{1}}\left(\mathcal{B}\left(I_{n-1}\right)\right)$.

By construction, we know that $\mathcal{B}^{2}\left(I_{n-1}\right) \backslash \mathcal{B} \mathcal{A}\left(I_{n-1}\right)$ is free from $\omega\left(c_{0}\right)$ and that the poscritical set of $\mathcal{B} \mathcal{A}\left(I_{n-1}\right)$ is contained in $\mathcal{A}^{2}\left(I_{n-1}\right)$. Moreover, the pairs 
$\mathcal{B}^{2}\left(I_{n-1}\right) \supset \mathcal{B} \mathcal{A}\left(I_{n-1}\right)$ and $\mathcal{B} \mathcal{A}\left(I_{n-1}\right) \supset \mathcal{A}^{2}\left(I_{n-1}\right)$ are both nice pairs. By the above, we have that $I_{n-1}$ is $\rho_{1}$-nice, Lemma 3.19 implies the existence of $\rho_{2}>0$ such that $\left(1+2 \rho_{2}\right) \mathcal{A}\left(I_{n-1}\right) \subset \mathcal{B}\left(I_{n-1}\right)$. Since $\mathcal{B}^{2}\left(I_{n-1}\right)$ is a pullback of bounded order (depending only on $b$ ) of $\mathcal{B}\left(I_{n-1}\right)$, Lemma 3.3 implies the existence of $\rho_{3}>0$ so that $\left(1+2 \rho_{3}\right) \mathcal{B} \mathcal{A}\left(I_{n-1}\right) \subset \mathcal{B}^{2}\left(I_{n-1}\right)$. By an analogous argument we get that $\left(1+2 \rho_{3}\right) \mathcal{A}^{2}\left(I_{n-1}\right) \subset \mathcal{B} \mathcal{A}\left(I_{n-1}\right)$. So $\mathcal{B} \mathcal{A}\left(I_{n-1}\right)$ is internally and externally free. Since $I_{n}$ is a pullback of bounded order of $\mathcal{B} \mathcal{A}\left(I_{n-1}\right)$, the result follows from Lemma 3.19. Observe that the external free space is given by $J_{n}=\Gamma^{T}\left(\mathcal{B}^{2}\left(I_{n-1}\right)\right)$, the internal free space is given by $J_{n}^{\prime}=\Gamma^{T}\left(\mathcal{A}^{2}\left(I_{n-1}\right)\right)$, and both $J_{n} \supset I_{n}$ and $I_{n} \supset J_{n}^{\prime}$ are nice pairs.

Finally, if $c_{0}$ is even the components of $I_{n} \backslash J_{n}^{\prime}$ are comparable to $\left|I_{n}\right|$. If $c_{0}$ is odd, then by the 2nd part of Lemma 3.19 there exists $\rho_{4}(v)>0$ so that if $\left|I_{n-1}\right| /\left|I_{n}\right|<v$, $\left|J_{n}^{\prime}\right| \geq \rho_{4}\left|I_{n}\right|$.

Part (b). Since $I_{n}$ is non-terminating, we know that $\mathcal{R}\left(I_{n-1}\right)$ is non-terminating. By Proposition 3.20 (3) there exists $\rho>0$ so that $\mathcal{R}\left(I_{n-1}\right)$ is $\rho$-free. The result follows from the definition of $I_{n}$ and Corollary 3.5.

Part (c). By Lemma 3.12, $\left(1+2 C^{\prime}\right) \mathcal{L}_{c_{0}}\left(\mathcal{L}_{x}\left(I_{n}\right)\right) \subset \mathcal{L}_{c_{0}}\left(I_{n}\right)$. Since $\Gamma\left(I_{n}\right) \subset \mathcal{L}_{c_{0}}\left(\mathcal{L}_{x}\left(I_{n}\right)\right)$, the result follows.

Part (d). The first part follows directly from Corollary 3.4. To show the second part, observe that if $I_{n}$ is $C$-nice, then by Lemma 3.3 there exists $C^{\prime \prime}>0, C^{\prime \prime} \rightarrow \infty$ as $C \rightarrow \infty$, so that $\left(1+2 C^{\prime \prime}\right) \mathcal{A}(I) \subset \mathcal{B}(I)$. So the result follows from Corollary 3.5.

Part (e). If $\left|I_{n+1}\right|<\varepsilon^{\prime} / 2\left|I_{n}\right|$ we are done, so let us assume this is not the case. Let us first assume that $I_{n-1}$ is terminating. For each $\varepsilon^{\prime \prime}>0$ there exists $C>0$ so that if $\left|I_{n}\right|<\varepsilon^{\prime \prime}\left|\mathcal{R}\left(I_{n-1}\right)\right|$, then $(1+2 C) I_{n} \subset \mathcal{R}\left(I_{n-1}\right)$. By Lemma 3.12 we know that $I_{n}$ is $C^{\prime}$-nice, for some $C^{\prime}(C)>0\left(C^{\prime} \rightarrow \infty\right.$ as $\left.C \rightarrow \infty\right)$. The result follows if $\varepsilon^{\prime \prime}$ is sufficiently small, so let us assume this is not the case. So there exists $v>0$ so that $\left|R\left(I_{n-1}\right)\right| /\left|I_{n}\right|,\left|I_{n}\right| /\left|I_{n+1}\right|<v$. By Proposition 3.20 and the definition of $I_{n}$, there exists $\rho>0$ such that $I_{n}$ is $\rho$-free. Then since $\left|\mathcal{L}_{x}\left(I_{n}\right)\right|<\varepsilon\left|I_{n}\right|$, there exists $C^{\prime \prime}>0\left(C^{\prime \prime} \rightarrow \infty\right.$ as $\left.\varepsilon \rightarrow 0\right)$ such that $\left(1+2 C^{\prime \prime}\right) \mathcal{L}_{x}\left(I_{n}\right) \subset I_{n}$ and the statement follows by Part (c). A similar argument works when $I_{n-1}$ is non-terminating and $c_{0}$ is even. Assume $I_{n-1}$ is non-terminating and $c_{0}$ is odd, then by Part (a) $I_{n}$ is $\rho$-nice and $\rho$-externally free. The second part of Lemma 3.12 implies that taking $\varepsilon$ sufficiently small $\left|\mathcal{L}_{c_{0}}\left(\mathcal{L}_{x}\left(I_{n}\right)\right)\right| \leq \epsilon^{\prime}\left|\mathcal{L}_{c_{0}}\left(I_{n}\right)\right|$, and the result follows.

In order to prove the remaining part of the theorem we will need two extra lemmas, and the following definition. We say that an interval $J \subset I$ is $\varepsilon$-small in $I$ if $|J| \leq \varepsilon|I|$.

Lemma 3.23. Given $\varepsilon \in(0,1), \rho>0$ and $N \in \mathbb{N}$ exists $\varepsilon^{\prime}>0$ so that the following holds. Assume that $c_{0}$ is odd and that I is a $\rho$-nice interval with $((1+2 \rho) I \backslash I) \cap \omega\left(c_{0}\right)=\emptyset$. Let $J \subset I$ be a pullback of I of order $N$ with $c_{0} \in J$. If

$$
\frac{|J|}{|I|}<\varepsilon^{\prime},
$$

then for each $x \in J \cap \omega\left(c_{0}\right)$ we have that $\mathcal{L}_{x}(J)$ is $\varepsilon$-small compared to $J$.

Proof. Let us begin by showing the following claim.

Claim. For each $v>0$ there exists $v^{\prime}>0$ such that if $J$ is $v^{\prime}$-small in $I$ then for any $y \in \omega\left(c_{0}\right), \mathcal{L}_{y}(J)$ is $v$-small in $\mathcal{L}_{y}(I)$. 
Proof of claim. Let $y \in \omega\left(c_{0}\right)$. There are two cases. Let us first assume $J \supset \mathcal{L}_{z}(I)$ for some $z \in \omega\left(c_{0}\right)$. Then, the claim follows directly form Lemma 3.14. So assume there exists $z \in \omega\left(c_{0}\right)$ so that $J \subset \mathcal{L}_{z}(I)$. If $J$ is comparable to $\mathcal{L}_{z}(I)$, then $\mathcal{L}_{z}(I)$ is small in $I$ and the claim follows from Lemma 3.14 applied to $\mathcal{L}_{z}(I)$ and $I$, since $\mathcal{L}_{y}(J) \subset \mathcal{L}_{y}\left(\mathcal{L}_{z}(I)\right)$, and the landing domains to $\mathcal{L}_{z}(I)$ are small compared to the landing domains to $I$. Now, if $\mathcal{L}_{z}(I)$ is much larger than $J$, we get that $J$ is deep inside of $I$, and the claim follows from Lemma 3.15.

Let $\left\{G_{j}\right\}_{j=0}^{s}$ be the chain with $G_{s}=I$ and $G_{0}=J$. Let $x \in J \cap \omega\left(c_{0}\right)$ be fixed, and let $k$ be its first return time to $J$. Let us first assume $k \geq s$ and let $y=f^{s}(x)$. By the claim, $\mathcal{L}_{y}(J)$ is $\varepsilon^{\prime \prime}$-small compared to $\mathcal{L}_{y}(I)$, for some $\varepsilon^{\prime \prime}>0$ with $\varepsilon^{\prime \prime} \rightarrow 0$ as $\varepsilon^{\prime} \rightarrow 0$. Since $I$ is $\rho$-nice this implies $\mathcal{L}_{y}(J)$ is deep-inside $I$, and the result follows from Lemma 3.3.

Assume that $k<s$. Observe that by definition of $k, G_{k}$ contains $J$. Consider the times $k \leq s_{i}<s$ with $s_{0}=k$ and $s_{i}<s_{i+1}$ such that $J \subset G_{s_{i}}$. Recall there are at most $n<N$ such times. Our aim is to show that $J$ is small in $G_{k}$, so the proof follows form the argument above. We will do this by showing that $J$ is small compared to each $G_{S_{i}}$, for $i=0, \ldots n$. If $J$ is small compared to $G_{s_{n}}$ we are done for $n$, so let us assume $G_{S_{n}}$ is comparable to $J$. By the argument above we know that for any $z \in \omega\left(c_{0}\right) \cap G_{S_{n}}$ we have that $\mathcal{L}_{z}\left(G_{S_{n}}\right)$ is $\varepsilon_{n}$-small compared to $G_{S_{n}}$, with $\varepsilon_{n} \rightarrow 0$ as $\varepsilon^{\prime} \rightarrow 0$. In particular, $G_{S_{n-1}}$ is small compared to $G_{S_{n}}$, so $J$ is small compared to $G_{S_{n}}$, contradiction. So $J$ is small compared to $G_{s_{n}}$. Applying this argument $n-k$ times we show $J$ is $\varepsilon_{k}$-small in $G_{k}$, with $\varepsilon_{k} \rightarrow 0$ as $\varepsilon^{\prime} \rightarrow 0$, and the result follows.

Lemma 3.24. Suppose that $I_{n-1}$ and $I_{n}$ are non-terminating. Then for any $C>0$, there exists $\varepsilon>0$ such that if

$$
\frac{\left|I_{n}\right|}{\left|I_{n-1}\right|}<\varepsilon
$$

then

(1) $\Gamma\left(I_{n}^{1}\right)$ is $C$-nice;

(2) $I_{n+1}$ is $C$-strongly nice and $C$-externally free.

Proof. First we will show that (1) holds. If $c_{0}$ is a critical point of even order, then for any $C^{\prime}>0$, there exists $\varepsilon>0$ such that if $\left|I_{n}\right| /\left|I_{n-1}\right|<\varepsilon$, then $\left(1+2 C^{\prime}\right) I_{n} \subset I_{n-1}$. By Lemma 3.16, if $C^{\prime}$ is chosen large enough, then $I_{n}$ will be $C^{\prime \prime}$-nice. Finally, since $\Gamma\left(I_{n}^{1}\right)$ is a pullback of $I_{n}$ of bounded order (only depending on $\underline{b}$ ) we can apply Lemma 3.16 to prove (1). So from now on we will assume that $c_{0}$ is of odd order. Since $c_{0}$ is odd all intervals from the enhanced nest are non-terminating. In particular, by Theorem 3.1 (a) we have that there exists $\rho>0$ such that that $I_{n-1}$ and $I_{n}$ are both $\rho$-nice and $\rho$-externally free.

In particular,

$$
I_{n} \subset(1+2 \rho) I_{n} \subset \Gamma^{T} \mathcal{B}^{2}\left(I_{n-1}\right)=: F_{n} .
$$

By the proof Part (a) of Theorem 3.1, we know that $F_{n} \backslash I_{n}$ is disjoint from $\omega\left(c_{0}\right)$, and $I_{n} \subset F_{n}$ is a nice pair. By Lemma 3.23, every component of the return map to $I_{n}$ is $\varepsilon^{\prime}$-small in $I_{n}$, so we have that every component of the domain of the return map to $I_{n}$ that intersects $\omega\left(c_{0}\right)$, in particular $I_{n}^{1}$, is $C^{\prime}$-well-inside $F_{n}$ with $C^{\prime} \rightarrow \infty$ as $\varepsilon^{\prime} \rightarrow 0$.

To see that $\Gamma\left(I_{n}^{1}\right)$ is $C$-nice, let $x \in I_{n}^{1} \cap \omega\left(c_{0}\right)$. Note that by Lemma 3.14 we have that the return domains to $I_{n}^{1}$ are small compared to $I_{n}^{1}$. The first return map from $I_{n}^{1}$ 
to $I_{n}$ extends to a map from a domain $\tilde{J} \supset I_{n}^{1}$ to $F_{n}$. Since $F_{n} \backslash I_{n}$ is disjoint from $\omega\left(c_{0}\right),\left(\tilde{J} \backslash I_{n}^{1}\right) \cap \omega\left(c_{0}\right)=\emptyset$, the first return map from $\mathcal{L}_{x}\left(I_{n}^{1}\right)$ to $I_{n}^{1}$ extends to a map from $J^{\prime} \supset \mathcal{L}_{x}\left(I_{n}^{1}\right)$ to $\tilde{J}$, and there exists a constant $C_{1}, C_{1} \rightarrow \infty$ as $\varepsilon \rightarrow 0$ such that $\left(1+2 C_{1}\right) \mathcal{L}_{x}\left(I_{n}^{1}\right) \subset J^{\prime}$. Since $I_{n}^{1}$ is non-terminating, we have that $J^{\prime} \subset I_{n}^{1}$, for all but one component: the component of the domain of the return map to $I_{n}^{1}$ that has the same return time to $I_{n}^{1}$ as $I_{n}^{1}$ has to $I_{n}$. For this one component, $J^{\prime}=\tilde{J}$. As in Lemma 3.18, there exists $C, C \rightarrow \infty$ as $C_{1} \rightarrow \infty$, such that $\Gamma\left(I_{n}^{1}\right)$ is $C$-nice.

Now we show that (2) holds. Observe that the map $f^{v}: \mathcal{B}\left(I_{n}\right) \rightarrow I_{n}$ extends to a domain $\tilde{F}_{n}$, so that $f^{v}: \tilde{F}_{n} \rightarrow F_{n}$ has the same critical points as $f^{v} \mid \mathcal{B}\left(I_{n}\right)$.

By Lemma 3.23, we know that the landing domains to $I_{n}$ are small compared to $I_{n}$, which implies that $\mathcal{A}\left(I_{n}\right)$ is $C^{\prime}$-well-inside $\tilde{F}_{n}$, for some $C^{\prime}>0$ with $C^{\prime} \rightarrow \infty$ as $\varepsilon \rightarrow 0$. In addition, $\tilde{F}_{n} \backslash \mathcal{A}\left(I_{n}\right)$ is disjoint from $\omega\left(c_{0}\right)$. If we let $H_{n+1}:=\Gamma^{T} \mathcal{B}\left(\tilde{F}_{n}\right)$, then $H_{n+1} \backslash I_{n+1}$ is disjoint from $\omega\left(c_{0}\right)$. By Lemma 3.3 there exists $C^{\prime \prime}>0$ so that $\left(1+2 C^{\prime \prime}\right) I_{n+1} \subset H_{n+1}$, where $C^{\prime \prime} \rightarrow \infty$ as $C^{\prime} \rightarrow \infty$. So $I_{n+1}$ is $C^{\prime \prime}$-externally free. Observe that the return time of any $x \in \omega\left(c_{0}\right) \cap I_{n+1}$ to $I_{n+1}$ is the same as its return time to $H_{n+1}$. Thus, there exists a constant $C>0, C \rightarrow \infty$ as $C^{\prime \prime} \rightarrow \infty$, such that for each $x \in \omega\left(c_{0}\right) \cap H_{n+1}$ we have that

$$
\mathcal{L}_{x}\left(I_{n+1}\right) \subset(1+2 C) \mathcal{L}_{x}\left(I_{n+1}\right) \subset \mathcal{L}_{x}\left(H_{n+1}\right) \subset I_{n+1}
$$

Moreover, each component of the domain of the return map to $H_{n}$ that intersects $\omega\left(c_{0}\right)$ contains a unique component of the domain of the return map to $I_{n+1}$ that intersects $\omega\left(c_{0}\right)$. Hence $I_{n+1}$ is $C$-strongly nice, where $C \rightarrow \infty$ as $\varepsilon \rightarrow 0$.

Now we can prove the remaining part of Theorem 3.1 .

Part (f). The fact that $I_{n+2}$ is $C^{\prime}$-strongly nice and $C^{\prime}$-externally free follows from Lemma 3.24.

Part (g). By Lemma 3.15, there exists $C^{\prime \prime}>0\left(C^{\prime \prime} \rightarrow \infty\right.$ as $\left.C \rightarrow \infty\right)$ such that if $(1+2 C) I_{n+1} \subset I_{n}$, then $\left(1+2 C^{\prime \prime}\right) I_{n+1}^{1} \subset I_{n+1}$. The result follows from this and the fact that $\omega\left(c_{0}\right) \cap I_{n+1} \subset I_{n+1}^{\infty}$.

3.7. Existence of free space. As a corollary to Theorem 3.1 and Proposition 3.20, we have the following:

Corollary 3.25. For each $v>0$ there exists $\rho>0$ such that if $I_{i}$ is an interval from the enhanced nest, then

(1) if $I_{i-1}$ is terminating and $\left|I_{i-1}\right| /\left|I_{i+2}\right|<v$, then $I_{i}$ is $\rho$-free;

(2) if $I_{i-1}$ and $I_{i}$ are non-terminating, and $\left|I_{i-1}\right| /\left|I_{i}\right|<\nu$, then $I_{i}$ is $\rho$-free;

(3) if $I_{i-1}$ is non-terminating and $I_{i}$ is terminating, then $I_{i}$ is $\rho$-free.

Proof. The first part follows from Proposition 3.20 and Lemma 3.3, and the second and third from Theorem 3.1. 


\section{Poincaré Disks and Tools for Dealing with Analytic Maps}

To construct box mappings we will make use of Poincaré disks. In this section, we study the basic properties of Poincaré disks and their pullbacks under analytic maps, so throughout this section we consider maps $f \in \mathcal{A}_{\underline{b}}$. An analytic map $f$ on the interval extends uniquely to a holomorphic mapping on a neighbourhood of the interval, and we will also use $f$ to denote this extension. We recall that given an interval $I$, the Poincaré disk with angle $\theta$ based on $I$ is denoted by $D_{\theta}(I)$. For a definition, see Sect. 1.10, p. 17.

Lemma 4.1 (Almost Schwarz Inclusion [dFdM2]). There exist $K<\infty, a_{0}>0$ and $a$ function $\theta:\left(0, a_{0}\right) \rightarrow(0, \infty)$ satisfying $\theta(a) \rightarrow 0$ and $a / \theta(a) \rightarrow 0$ as $a \rightarrow 0$ such that the following holds. Let $F: \mathbb{D} \rightarrow \mathbb{C}$ be univalent and real-symmetric, and assume that $I \subset \mathbb{R}$ is an interval containing 0 with $|I|<a \in\left(0, a_{0}\right)$. Let $I^{\prime}=F(I)$. Then

(a) for all $\theta \geq \theta(|I|)$, we have

$$
F\left(D_{\theta}(I)\right) \subset D_{\left(1-K|I|^{1+\delta}\right) \theta}\left(I^{\prime}\right),
$$

where $0<\delta<1$ is a universal constant;

(b) for all $\theta \in(\pi / 2, \pi)$ we have

$$
F\left(D_{\pi-\theta}(I)\right) \subset D_{\pi-K|I| \theta}\left(I^{\prime}\right) .
$$

Lemma 4.2 (cf. [S3], Lemma 7.4). Let $\ell \geq 2$ be an integer and consider $P(z)=z^{\ell}$. Then, for each $\theta \in(0, \pi)$ the following holds.

- Suppose $\ell$ is even and let $K \geq 1$. Then there exists $\lambda=\lambda(K, \ell) \in(0,1)$ such that

$$
\left.P^{-1}\left(D_{\theta}(-K, 1)\right) \subset D_{\lambda \theta}(-1,1)\right) \text {. }
$$

- Suppose that $\ell$ is an odd integer. Let $K>0$. Then there exists $\lambda=\lambda(\ell) \in(0,1)$ such that

$$
P^{-1}\left(D_{\theta}\left(-K^{\ell}, 1\right)\right) \subset D_{\lambda \theta}((-K, 1)) .
$$

Proof. We will give the proof in the case that $\ell$ is odd; the proof in the even case is similar. Since the problem is invariant under scaling, we can assume that $K \geq 1$. We will argue by contradiction. If the lemma is false, then for every $\lambda>0$, there exist $\theta_{1 / \lambda} \in(0, \pi)$ and $z_{1 / \lambda}^{\ell} \in D_{\theta}\left(-K^{\ell}, 1\right)$ such that $z_{1 / \lambda} \notin D_{\lambda \theta}(-K, 1)$. Note that we may as well assume that $\theta \in(0, \pi / 2)$, so that is what we will do. For each integer $n \geq 1$, let $\lambda_{n}=1 / n$ and let $\theta_{n}$ and $z_{n}$ be the corresponding $\theta_{1 / \lambda}$ and $z_{1 / \lambda}$.

Since $z_{n} \notin D_{\theta_{n} / n}(-K, 1)$,

$$
\arg \frac{z_{n}-1}{z_{n}+K} \leq \frac{\theta_{n}}{n}
$$

so that

$$
\tan \arg \frac{z_{n}-1}{z_{n}+K} \leq \tan \frac{\theta_{n}}{n} .
$$

Writing $z_{n}=r_{n} e^{i t_{n}}$, we have

$$
\arg \frac{z_{n}-1}{z_{n}+K}=\arg \left(z_{n}-1\right)-\arg \left(z_{n}+K\right)
$$




$$
=\arctan \left(\frac{r_{n} \sin t_{n}}{r_{n} \cos t_{n}-1}\right)-\arctan \left(\frac{r_{n} \sin t_{n}}{r_{n} \cos t_{n}+K}\right)
$$

So, taking the tangent gives us

$\frac{r_{n} \sin t_{n}(K+1)}{r_{n}^{2}+r_{n}(-1+K) \cos t_{n}-K}=\frac{\frac{r_{n} \sin t_{n}}{r_{n} \cos t_{n}-1}-\frac{r_{n} \sin t_{n}}{r_{n} \cos t_{n}+K}}{1+\left(\frac{r_{n} \sin t_{n}}{r \cos t_{n}-1}\right)\left(\frac{r_{n} \sin t_{n}}{r_{n} \cos t_{n}+K}\right)}=\tan \arg \frac{z_{n}-1}{z_{n}+K} \leq \tan \frac{\theta_{n}}{n}$.

Similarly, letting $w_{n}=z_{n}^{\ell}=r_{n}^{\ell} e^{i \ell t_{n}}$, we have

$$
\frac{r_{n}^{\ell} \sin \ell t_{n}\left(K^{\ell}+1\right)}{r_{n}^{2 \ell}+r_{n}^{\ell}\left(-1+K^{\ell}\right) \cos \ell t_{n}-K^{\ell}}=\tan \arg \frac{w_{n}-1}{w_{n}+K^{\ell}} \geq \tan \left(\theta_{n}\right) .
$$

Dividing the second inequality by the first one, we have that

$$
\frac{r_{n}^{\ell} \sin \ell t_{n}\left(K^{\ell}+1\right)\left(r_{n}^{2}+r_{n}(-1+K) \cos t_{n}-K\right)}{\left(r_{n}^{2 \ell}+r_{n}^{\ell}\left(-1+K^{\ell}\right) \cos \ell t_{n}-K^{\ell}\right) r_{n} \sin t_{n}(K+1)} \geq \frac{\tan \theta_{n}}{\tan \left(\theta_{n} / n\right)}
$$

Observe this expression becomes very large as $n \rightarrow \infty$. Since $\sin \ell t_{n} / \sin t_{n}$ is bounded from above, and cancelling an $r_{n}$ in the numerator and denominator, this is equivalent to

$$
\frac{r_{n}^{\ell-1}\left(K^{\ell}+1\right)\left(r_{n}^{2}+r_{n}(-1+K) \cos t_{n}-K\right)}{\left(r_{n}^{2 \ell}+r_{n}^{\ell}\left(-1+K^{\ell}\right) \cos \ell t_{n}-K^{\ell}\right)(K+1)} \asymp \frac{r_{n}^{\ell+1}+r_{n}^{\ell}(K-1) \cos t_{n}-r_{n}^{\ell-1} K}{r_{n}^{2 \ell}+r_{n}^{\ell}\left(-1+K^{\ell}\right) \cos \ell t_{n}-K^{\ell}}
$$

becoming very large as $n$ tends to infinity. We will show that this is impossible.

Let $R_{n}=r_{n} / K$. Equation 4.2 is comparable to

$$
\frac{r_{n}^{\ell+1} K^{\ell-1}+r_{n}^{\ell} K^{\ell} \cos t_{n}-r_{n}^{\ell} K^{\ell-1} \cos t_{n}-r_{n}^{\ell-1} K^{\ell}}{r_{n}^{2 \ell}+r_{n}^{\ell} K^{\ell} \cos \ell t_{n}-r_{n}^{\ell} \cos \ell t_{n}-K^{\ell}},
$$

which equals

$$
\frac{\frac{r_{n}^{\ell+1}}{K^{\ell+1}}+\frac{r_{n}^{\ell}}{K^{\ell}} \cos t_{n}-\frac{r_{n}^{\ell} \cos t_{n}}{K^{\ell+1}}-\frac{r_{n}^{\ell-1}}{K^{\ell}}}{\frac{r_{n}^{2 \ell}}{K^{2 \ell}}+\frac{r_{n}^{\ell}}{K^{\ell}} \cos \ell t_{n}-\frac{r_{n}^{\ell} \cos \ell t_{n}}{K^{2 \ell}}-\frac{1}{K^{\ell}}}=\frac{R_{n}^{\ell+1}+R_{n}^{\ell} \cos t_{n}-\frac{R_{n}^{\ell}}{K} \cos t_{n}-\frac{R_{n}^{\ell-1}}{K}}{R_{n}^{2 \ell}+R_{n}^{\ell} \cos \ell t_{n}-\frac{R_{n}^{\ell}}{K^{\ell}} \cos \ell t_{n}-\frac{1}{K^{\ell}}}
$$

in the scaled coordinates. Since this expression becomes very large as $n \rightarrow \infty$, by comparing powers of $R_{n}$, we see that $R_{n}$ is bounded. Since $z_{n} \notin D_{\theta_{n} / n}(-K, 1)$, we have that $t_{n} \rightarrow 0$ or $t_{n} \rightarrow \pi$. First consider the case that $t_{n} \rightarrow 0$. Then as $n \rightarrow \infty$,

$$
\arg \left(\frac{z_{n}-1}{z_{n}+K}\right) \asymp \arg \left(z_{n}-1\right) \text { and } \arg \left(\frac{z_{n}^{\ell}-1}{z_{n}^{\ell}+K^{\ell}}\right) \asymp \arg \left(z_{n}^{\ell}-1\right) \text {. }
$$

However, since $z_{n} \notin D_{\theta_{n} / n}(-K, 1)$ and $z_{n}^{\ell} \in D_{\theta_{n}}\left(-K^{\ell}, 1\right)$, combining these estimates with (4.1) we have that

$$
\frac{\arg \left(z_{n}^{\ell}-1\right)}{\arg \left(z_{n}-1\right)} \rightarrow \infty
$$


We will show that this is impossible. Suppose that $r_{n}$ does not converge to 1 . Then, since $t_{n} \rightarrow 0$, neither $r_{n}^{\ell} \cos \left(\ell t_{n}\right)-1$ nor $r_{n} \cos \left(t_{n}\right)-1$ converges to 0 . But now we see that

$$
\frac{\arg \left(z_{n}^{\ell}-1\right)}{\arg \left(z_{n}-1\right)}=\frac{\arctan \frac{r_{n}^{\ell} \sin \left(\ell t_{n}\right)}{r_{n}^{\ell} \cos \left(\ell t_{n}\right)-1}}{\arctan \frac{r_{n} \sin \left(t_{n}\right)}{r_{n} \cos \left(t_{n}\right)-1}} \asymp \frac{\frac{r_{n}^{\ell} \sin \left(\ell t_{n}\right)}{r_{n}^{\ell} \cos \left(\ell t_{n}\right)-1}}{\frac{r_{n} \sin \left(t_{n}\right)}{r_{n} \cos \left(t_{n}\right)-1}} \asymp \frac{r_{n}^{\ell} \sin \left(\ell t_{n}\right)}{r_{n} \sin \left(t_{n}\right)}
$$

is bounded. So we can assume that $r_{n} \rightarrow 1$.

Then we have that

$$
\begin{aligned}
& \frac{\arg \left(z_{n}^{\ell}-1\right)}{\arg \left(z_{n}-1\right)}=\frac{\arg \left(\left(z_{n}-1\right)\left(z_{n}^{\ell-1}+z_{n}^{\ell-2}+\cdots+1\right)\right)}{\arg \left(z_{n}-1\right)} \\
& \quad=\frac{\arg \left(\left(z_{n}-1\right)+\arg \left(z_{n}^{\ell-1}+z_{n}^{\ell-1}+\cdots+1\right)\right.}{\arg \left(z_{n}-1\right)}=1+\frac{\arg \left(z_{n}^{\ell-1}+z_{n}^{\ell-1}+\cdots+1\right)}{\arg \left(z_{n}-1\right)},
\end{aligned}
$$

which is bounded, since there exists a constant $C>0$ such that

$$
\arg \left(z_{n}^{\ell-1}+z_{n}^{\ell-1}+\cdots+1\right) \leq C \arg \left(z_{n}-1\right)
$$

as $z_{n} \rightarrow 1$.

Now, suppose that $t_{n} \rightarrow \pi$. Then as $n \rightarrow \infty$,

$$
\arg \left(\frac{z_{n}-1}{z_{n}+K}\right) \asymp \pi-\arg \left(z_{n}+K\right), \text { and } \arg \left(\frac{z_{n}^{\ell}-1}{z_{n}^{\ell}+K^{\ell}}\right) \asymp \pi-\arg \left(z_{n}^{\ell}+K^{\ell}\right) \text {. }
$$

But now we have that as $n \rightarrow \infty$

$$
\frac{\pi-\arg \left(z_{n}^{\ell}+K^{\ell}\right)}{\pi-\arg \left(z_{n}+K\right)} \rightarrow \infty
$$

but arguing just as we did in the case when $t_{n} \rightarrow 0$ we see that this is impossible. Suppose that $r_{n}$ does not converge to $-K$. Then, since $t_{n} \rightarrow \pi$, neither $K^{\ell}-r_{n}^{\ell} \cos \left(\ell t_{n}\right)$ nor $K-r_{n} \cos \left(t_{n}\right)$ converges to 0 . But now we see that

$$
\frac{\pi-\arg \left(z_{n}^{\ell}+K^{\ell}\right)}{\pi-\arg \left(z_{n}+K\right)}=\frac{\arctan \frac{r_{n}^{\ell} \sin \left(\ell t_{n}\right)}{K^{\ell}-r_{n}^{\ell} \cos \left(\ell t_{n}\right)}}{\arctan \frac{r_{n} \sin \left(t_{n}\right)}{K-r_{n} \cos \left(t_{n}\right)}} \asymp \frac{\frac{r_{n}^{\ell} \sin \left(\ell t_{n}\right)}{K^{\ell}-r_{n}^{\ell} \cos \left(\ell t_{n}\right)}}{\frac{r_{n} \sin \left(t_{n}\right)}{K-r_{n} \cos \left(t_{n}\right)}} \asymp \frac{r_{n}^{\ell} \sin \left(\ell t_{n}\right)}{r_{n} \sin \left(t_{n}\right)}
$$

is bounded. So we can assume that $r_{n} \rightarrow-K$. Now, a similar calculation to the one in (4.3) shows that

$$
\frac{\pi-\arg \left(z_{n}^{\ell}+K^{\ell}\right)}{\pi-\arg \left(z_{n}+K\right)}
$$

must be bounded, which again yields a contradiction.

The proof when $\ell$ is even is similar and is a natural generalization of [S3, Lemma 7.4], see [KSvS, Lemma 13.2].

The next lemma is straight forward.

Lemma 4.3 (cf. [KSvS], Lemma 13.3). Let $\ell \geq 2$ be an integer and consider $P(z)=z^{\ell}$. For any $A>0$ and any $\theta \in(0, \pi)$, there exists $\theta^{\prime} \in(0, \pi)$ such that the following holds. 
- If $\ell$ is even, then

$$
P^{-1}\left(D_{\theta}((-A, 1))\right) \supset D_{\theta^{\prime}}((-1,1)) .
$$

- If $\ell$ is odd, then there exists $\theta^{\prime}$ such that

$$
P^{-1}\left(D_{\theta}\left(\left(-A^{\ell}, 1\right)\right)\right) \supset D_{\theta^{\prime}}((-A, 1)) .
$$

Although we state the next lemma for any integer $\ell \geq 2$, it will only be used to deal with inflection points.

Lemma 4.4. Let $\ell \geq 2$ be an integer and consider $P(z)=z^{\ell}$. For each $C<\infty$, there exists $\lambda \in(0,1)$, depending on $\ell$ and $C$, so that for all $\theta \in(0, \pi / 2)$ the following holds. Let $I_{1} \subset[0,1)$ and $I_{2} \subset(-1,0]$ be intervals and assume that

$$
\left|I_{1}\right| \geq 1 / C,
$$

and that $w \in D_{\theta}\left(I_{2}\right)$. Let $z \in P^{-1}(w)$ be so that $|\arg (z)|<\pi / \ell$. Then

$$
z \in D_{\lambda \theta}\left(I_{1}\right) \cup A
$$

where $A=\emptyset$ if $0 \in I_{1}$ and otherwise $A$ is the component bounded by the lines $\arg (z)=$ $\pm \pi / \ell$ and the boundary of $D_{\lambda \theta}\left(I_{1}\right)$.

Proof. Fix $\theta<\pi / 4$. Let $I_{1}=(\alpha, \beta)$ and take $z \in \partial D_{\lambda \theta}(\alpha, \beta)$. Setting $z=r e^{i t}$ and arguing as in the proof of Lemma 4.2 , we have that

$$
\tan \arg \frac{z-\beta}{z-\alpha}=\frac{r(\beta-\alpha) \sin t}{r^{2}-r(\beta+\alpha) \cos t+\beta \alpha}=\tan \lambda \theta .
$$

Hence,

$$
r^{2}+\left(-(\beta+\alpha) \cos t-\frac{(\beta-\alpha)}{\tan \lambda \theta} \sin t\right) r+\alpha \beta=0
$$

This equation has two solutions,

$$
r_{ \pm}=\frac{1}{2}\left(\left((\alpha+\beta) \cos t+\frac{(\beta-\alpha) \sin t}{\tan \lambda \theta}\right) \pm \sqrt{\left((\alpha+\beta) \cos t+\frac{(\beta-\alpha) \sin t}{\tan \lambda \theta}\right)^{2}-4 \alpha \beta}\right) .
$$

Given $t \in(0, \pi / \ell)$ we denote by $z_{ \pm}=r_{ \pm} e^{i t}$. To prove the lemma, it suffices to show that we can take $\lambda>0$ small so that for all $t \in(0, \pi / \ell)$ we have $z_{+}^{\ell} \notin D_{\theta}(-1,0)$, see Fig. 5. This is equivalent to showing that

$$
\tan \arg \frac{z_{+}^{\ell}}{z_{+}^{\ell}+1} \leq \tan \theta, \text { i.e. } r_{+}^{\ell} \geq \frac{\sin (\ell t)}{\tan \theta}-\cos (\ell t) .
$$

From the equation for $r_{+}$we have

$$
r_{+}>\frac{1}{2}(\beta+\alpha) \cos t+\frac{\beta-\alpha}{2 \tan \lambda \theta} \sin t
$$

Hence

$$
r_{+}^{\ell} \geq \frac{1}{2^{\ell-1}}(\beta+\alpha)^{\ell-1} \cos ^{\ell-1} t \frac{\beta-\alpha}{2 \tan \lambda \theta} \sin t+\frac{(\beta-\alpha)^{\ell}}{2^{\ell} \tan ^{\ell} \lambda \theta} \sin ^{\ell} t .
$$



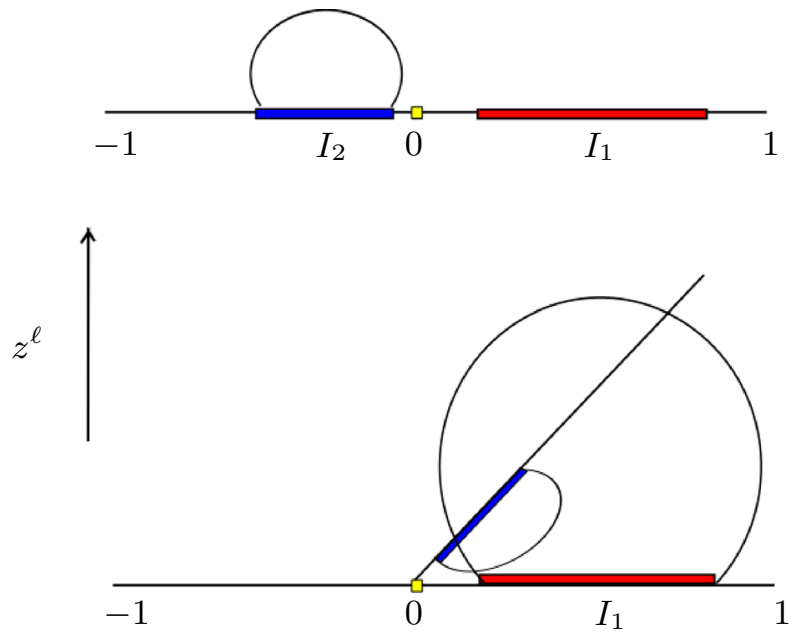

Fig. 5. The pullback of Poincaré disks as in Lemma 4.4

Provided we take $\lambda>0$ sufficiently small (here $\lambda$ depends on $\beta-\alpha$ and $\ell$ ), for each $t \in(0, \pi / \ell)$ the right hand side of this expression dominates the r.h.s. of (4.4). Indeed, if $\cos (t)$ is not small (which holds automatically if $\ell>2$, because $t \in(0, \pi / \ell)$ ), then we the first and second term in the r.h.s. of (4.5) dominate respectively the first and second term in the r.h.s. of (4.4) (provided we choose $\lambda>0$ small). If $\cos (t)$ is small, then $\sin (t)$ is not small, and the 2 nd term in (4.5) dominates the r.h.s. of (4.4), provided we choose $\lambda>0$ small.

Lemma 4.5 [KSvS, Lemma 13.4]. One can compare Poincaré disks in the following ways.

(a) There exists $\theta_{0}$ such that for each $A>1$ and each $\theta \in(0, \pi)$,

$$
D_{\theta}([-1,1]) \subset D_{\min \left\{\theta A / 2, \theta_{0}\right\}}([-A, A]) .
$$

(b) For each $\lambda \in(0,1)$ and $\delta>0$ there exists $\lambda^{\prime} \in(0,1)$ such that for each $\theta \in(0, \pi)$,

$$
D_{\lambda \theta}([-1,1]) \backslash D_{\theta}(-1-\delta, 1+\delta) \subset D_{\lambda^{\prime} \theta}([-1-\delta,-1]) .
$$

(c) For each $\lambda \in(0,1)$ and $\delta>0$ there exists $\lambda^{\prime} \in(0,1)$ such that for each $\theta \in(0, \pi)$

$$
D_{\lambda \theta}([-1,1]) \backslash D_{\theta}([-1-\delta, 1+\delta]) \subset D_{\lambda^{\prime} \theta}([1,1+\delta])
$$

(d) For each $\lambda \in(0,1)$ and $\delta>0$ there exists $\lambda^{\prime}>0$ such that for each interval $J \subset[-1,1]$ and each $\theta>0$,

$$
\left.D_{\lambda \theta}([-1,1]) \backslash D_{\theta}(-1-\delta, 1+\delta]\right) \subset D_{\lambda^{\prime} \theta|J|}(J)
$$


4.1. Pulling back Poincaré disks under disjoint orbits. In this subsection we will show how to pull back Poincaré disks along a chain of intervals under a map $f \in \mathcal{A}_{b}$. We remind the reader that we always assume that the Standing Assumptions on p. 26 hold.

Lemma 4.6 (cf. [KSvS], Lemma 13.5). For any $\delta>0$ there exists $\lambda>0$ and for each $\theta \in(0, \pi)$ there exists $\varepsilon>0$ so that following holds. Let $\left\{H_{j}\right\}_{j=0}^{s}$ and $\left\{H_{j}^{\prime}\right\}_{j=0}^{s}$ be two chains with $H_{j} \subset H_{j}^{\prime}$ for all $j=0, \ldots, s$ and $H_{0} \cap \omega\left(c_{0}\right) \neq \emptyset$. Assume that the following hold:

(a) $\left|H_{s}^{\prime}\right| \leq \varepsilon$,

(b) $H_{s}^{\prime} \supset(1+2 \delta) H_{s}$,

(c) the chain $\left\{H_{j}^{\prime}\right\}_{j=0}^{s-1}$ is disjoint,

(d) $H_{i} \backslash(1+2 \delta)^{-1} H_{i}, i=0, \ldots, s-1$ does not contain a critical point.

Let $U_{s}=D_{\theta}\left(H_{s}\right)$, and $U_{0}=\operatorname{Comp}_{H_{0}}\left(f^{-s}\left(U_{s}\right)\right)$. Then

$$
U \subset D_{\lambda \theta}\left(H_{0}\right) \text {. }
$$

Proof. The Lebesgue measure of $\omega\left(c_{0}\right)$ is zero (see [vSV, Theorem E]), so if $H_{s}^{\prime}$ is sufficiently small the set $\cup_{i=0}^{s} H_{i}^{\prime}$ is a small neighbourhood of a subset of $\omega\left(c_{0}\right)$, and we can assume that $\sum_{i=0}^{s}\left|H_{i}^{\prime}\right|$ is small.

First suppose that only $H_{0}^{\prime}$ contains a critical point. By the Improved Koebe Principle, Theorem 3.2 (2), we can find $\rho_{1}, \delta_{1}>0$ such that $H_{j}^{\prime} \supset\left(1+2 \rho_{1}\right) H_{j}$ and $H_{j} \backslash(1+$ $\left.2 \delta_{1}\right)^{-1} H_{j}$ contains no critical points of $f$ for $1 \leq j \leq s$. By Lemma 4.1 there exists $\lambda_{1}>0$ such that

$$
U_{1} \subset D_{\lambda_{1} \theta}\left(H_{1}\right) .
$$

Since $H_{1} \backslash\left(1+2 \delta_{1}\right)^{-1} H_{1}$ contains no critical values of $f$ we can apply Lemma 4.2 to find $\lambda_{2}>0$ such that

$$
U_{0} \subset D_{\lambda_{2} \theta}\left(H_{0}\right) .
$$

Since the critical points of $f$ are non-flat, there exists $\delta_{2}>0$ such that $H_{0}^{\prime} \supset\left(1+2 \delta_{2}\right) H_{0}$, and $\left(H_{0} \backslash\left(1+2 \delta_{2}\right)^{-1} H_{0}\right) \cap \mathrm{Crit}(f)=\emptyset$. Thus the lemma follows by applying the previous argument at most $b$ times.

This lemma implies the following corollaries.

Corollary 4.7. Suppose that instead of $(d)$ in the above lemma there exists $\delta^{\prime} \in(0, \delta)$ so that the sets $\left(1+2 \delta^{\prime}\right) H_{j} \backslash H_{j}$ are free from critical points for all $j \in\{0, \ldots s\}$. Then there exist an interval $H^{\prime}, \lambda^{\prime} \in(0,1)$ and $\delta^{\prime \prime}>0$ such that

$$
\hat{U}=\operatorname{Comp}_{H_{0}} f^{-s}\left(D_{\theta}\left(H_{S}\right)\right) \subset D_{\lambda^{\prime} \theta}\left(H^{\prime}\right)
$$

where $H_{0} \subset H^{\prime} \subset\left(1+2 \delta^{\prime \prime}\right) H^{\prime} \subset H_{0}^{\prime}$.

Corollary 4.8. For each $\delta>0$ there exist $\delta^{\prime}>0$ and $\lambda \in(0,1)$, and for each $\theta \in(0, \pi)$ there exists $\varepsilon^{\prime}>0$ such that the following holds for all $\varepsilon \in\left(0, \varepsilon^{\prime}\right)$. Let I be a nice interval with $|I|<\varepsilon$ and let be $J$ a domain of the first entry map to I with first entry time equal to $s>0$. Let $\left\{H_{j}\right\}_{j=0}^{s}$ be a chain with $(1+2 \delta) H_{s} \subset I, H_{0} \subset J$ and $H_{0} \cap \omega\left(c_{0}\right) \neq \emptyset$. Then there exists an interval $H^{\prime}$ with $H_{0} \subset H^{\prime} \subset\left(1+2 \delta^{\prime}\right) H^{\prime} \subset J$ so that

$$
\operatorname{Comp}_{H_{0}} f^{-s}\left(D_{\theta}\left(H_{s}\right)\right) \subset D_{\lambda \theta}\left(H^{\prime}\right) \text {. }
$$




\section{Tools for Dealing with $C^{3}$ Maps}

In this section, we will develop the additional tools required for dealing with $C^{3}$ maps. A reader who is primarily interested in the real analytic case can skip this section.

5.1. Quasiconformal and quasiregular mappings. We will find it convenient to make use of the analytic definition of quasiconformal and quasiregular mappings, since this approach facilitates the definition of quasiregular mappings. We refer the reader to [AIM] for additional background on these classes of mappings.

We define

$$
\partial_{\alpha} f(z)=\cos (\alpha) f_{x}(z)+\sin (\alpha) f_{y}(z), \quad \alpha \in[0,2 \pi) .
$$

Suppose that $U$ and $V$ are domains in $\mathbb{C}$. A mapping $f: U \rightarrow V$ is called $\kappa$-quasiregular, abbreviated $\kappa-q r$, if it is orientation-preserving, $f$ is in the Sobolev space $W_{l o c}^{1,2}(U)$, and the directional derivatives satisfy

$$
\max _{\alpha}\left|\partial_{\alpha} f(z)\right| \leq \kappa \min _{\alpha}\left|\partial_{\alpha} f(z)\right|
$$

for almost every $z \in \Omega$. If, in addition $f$ is a homeomorphism, then $f$ is called $\kappa$ quasiconformal, abbreviated $\kappa-q c$.

We call the constant $\kappa$ the quasiconformal distortion of $f$.

This definition of quasiconformal mappings is equivalent to the following geometric definition. A mapping $f: U \rightarrow V$ is $\kappa$-quasiconformal if and only if for any annulus $A \subset U$,

$$
\frac{1}{\kappa} \bmod (A) \leq \bmod (f(A)) \leq \kappa \bmod (A) .
$$

We say that a mapping $f: U \rightarrow V$ is quasiregular (quasiconformal) if it is $\kappa$ quasiregular ( $\kappa$-quasiconformal) for some $\kappa$.

We assume that $f \in W_{l o c}^{1,2}(U)$ since it implies that $\operatorname{Jac}(f)=\left|f_{z}\right|^{2}-\left|f_{\bar{z}}\right|^{2}$ is locallyintegrable. For the reader who is familiar with qc mappings, but not qr mappings, let us remark that for homeomorphisms $f \in W_{l o c}^{1,1}(U)$, implies $f \in W_{l o c}^{1,2}(U)$; however, for quasiregular mappings it is not sufficient to assume only that $f \in W_{l o c}^{1,1}(U)$.

Recall that

$$
f_{z}=\partial f=\frac{\partial f}{\partial z}=\frac{1}{2}\left(\frac{\partial f}{\partial x}-i \frac{\partial f}{\partial y}\right), \quad \text { and } \quad f_{\bar{z}}=\bar{\partial} f=\frac{\partial f}{\partial \bar{z}}=\frac{1}{2}\left(\frac{\partial f}{\partial x}+i \frac{\partial f}{\partial y}\right) .
$$

Theorem 5.1. Suppose that $f: U \rightarrow V$ is a homeomorphism in $W_{l o c}^{1,2}(U)$ then $f$ is $\kappa$-quasiconformal if and only if

$$
\frac{\partial f}{\partial \bar{z}}(z)=\mu_{f}(z) \frac{\partial f}{\partial z}(z) \text { for almost every } z \in U,
$$

where $\mu_{f}$ is a bounded measurable function satisfying

$$
\left\|\mu_{f}\right\|_{\infty} \leq \frac{\kappa-1}{\kappa+1}<1 .
$$


The function $\mu_{f}$ is called the Beltrami coefficient of $f$.

If $f$ is a $\kappa$-quasiconformal mapping with Beltrami coefficient $\mu_{f}$, let $\kappa(f)$ be the minimal $\kappa$ so that the quasiconformal distortion of $f$ is bounded by $\kappa$. We have that

$$
\left\|\mu_{f}\right\|_{\infty}=\frac{\kappa(f)-1}{\kappa(f)+1} \text { and } \kappa(f)=\frac{1+\left\|\mu_{f}\right\|_{\infty}}{1-\left\|\mu_{f}\right\|_{\infty}} .
$$

We will drop the subscript on the Beltrami differential when it is clear to what mapping we are referring.

By Weyl's Lemma a 1-quasiconformal mapping is conformal and a 1-quasiregular mapping is holomorphic (see [AIM], Lemma A.6.10). The following are some basic properties of quasiconformal mappings.

Proposition 5.2. Let $f: U \rightarrow V$ be a $\kappa$-qc mapping onto $V$ and let $g: V \rightarrow \mathbb{C}$ be $a$ $\kappa^{\prime}$-qc mapping. Then

- $f$ is differentiable a.e. on $U$.

- $f^{-1}: V \rightarrow U$ is $\kappa-q c$.

- $g \circ f: U \rightarrow \mathbb{C}$ is $\kappa^{\prime} \kappa-q c$.

Quasiregular mappings can be factored into a qc mapping followed by a holomorphic mapping.

Proposition 5.3 (Stoilow factorization). Suppose that $f$ is quasiregular and defined on a simply connected domain $\Omega$. Then $f=h \circ g$. where $g: \Omega \rightarrow \Omega$ is quasiconformal and $h$ is holomorphic on $\Omega$.

It follows that if $f: U \rightarrow \mathbb{C}$ is a quasiregular mapping, then $f$ is open and discrete. Moreover, if $f: U \rightarrow \mathbb{C}$ is quasiregular, then there exists $k \in[0,1)$ such that $\left|f_{\bar{z}}\right| \leq$ $k\left|f_{z}\right|$ almost everywhere in $U$, and we have

$$
\frac{\partial f}{\partial \bar{z}}=\mu(z) \frac{\partial f}{\partial z}, \quad \mu_{f}:=\frac{f_{\bar{z}}}{f_{z}} \quad \text { with }\left\|\mu_{f}\right\|_{\infty} \leq k .
$$

5.2. Asymptotically holomorphic extensions of a $C^{3}$ mapping. Let $\mathcal{K} \neq \varnothing$ be a compact subset of $\mathbb{R}^{2}, U$ an open neighbourhood of $\mathcal{K}$ and $f: U \rightarrow \mathbb{C}$ a $C^{1}$ map. We say that $f$ is asymptotically holomorphic of order $t, t \geq 1$, on $\mathcal{K} \subset \mathbb{R}^{2}$ if for every $(x, y) \in \mathcal{K}$

$$
\frac{\partial}{\partial \bar{z}} f(x, y)=0
$$

and

$$
\frac{\frac{\partial}{\partial \bar{z}} f(x, y)}{d((x, y), \mathcal{K})^{t-1}} \rightarrow 0
$$

uniformly as $(x, y) \rightarrow \mathcal{K}$ for $(x, y) \in U \backslash \mathcal{K}$.

In our applications of asymptotically holomorphic extensions, $\mathcal{K}$ will be an interval contained in the real line. 
Lemma 5.4 [GSS2, Lemma 2.1]. Suppose that $f:[0,1] \rightarrow[0,1]$ is a $C^{3}$ mapping. Let $0=u_{0}<v_{0}=u_{1}<v_{1}=u_{2}<\cdots=u_{n}<v_{n}=1$ be a partition of the interval such that on each interval $\left(u_{i}, v_{i}\right), f$ is either a diffeomorphism, $h_{i}$, with $h_{i}^{\prime}\left(u_{i}\right), h_{i}^{\prime}\left(v_{i}\right) \neq 0$, or $f$ can be expressed in the form $f(x)=f\left(\zeta_{i}\right)\left(1-h_{i}(x)^{\ell}\right)$ for $\zeta_{i} \in\left(u_{i}, v_{i}\right) \cap \operatorname{Crit}(f)$ and $h_{i}$ is a diffeomorphism such that $h_{i}^{\prime}\left(u_{i}\right), h_{i}^{\prime}\left(v_{i}\right) \neq 0$, and $h_{i}\left(\zeta_{i}\right)=0$. In either case, the diffeomorphism $h_{i}$ has a $C^{3}$-extension to a diffeomorphism $H_{i}$ defined on a neighbourhood $U$ of the interval $\left[u_{i}, v_{i}\right]$ in the complex plane and $H_{i}$ is asymptotically holomorphic of order 3 on $\left[u_{i}, v_{i}\right]$ in $U$. Moreover, the neighbourhood $U$ and the extension $H_{i}$ are symmetric with respect to the real axis.

We will also use $f$ to denote the asymptotically holomorphic extension of order three of a map $f$, and whenever we refer to an asymptotically holomorphic extension we mean the asymptotically holomorphic extension of order three given by Lemma 5.4. We refer the reader to [GSS2] for background on asymptotically holomorphic extensions of real maps. Let us remark that in general $\kappa$-quasiregular mappings are only differentiable almost everywhere; however, in our setting, they are $C^{3}$.

An equivalent definition of $\kappa$-quasiconformal is the following: $H: U \rightarrow V$ is $\kappa$ quasiconformal iff $H \in W_{l o c}^{1,2}(U)$ and

$$
|D H(z)|^{2}<\kappa J(H, z),
$$

where $D H=H_{z}+H_{\bar{z}}$ and $J(H, z)=\left|H_{z}\right|^{2}-\left|H_{\bar{z}}\right|^{2}$. Direct calculation shows that if $H$ is an asymptotically holomorphic extension given by Lemma 5.4 at a point $x \in \mathbb{R}$, then $J(H, x)=\left|h^{\prime}(x)\right|^{2}$. Since each $h$ is a diffeomorphism, we can assume that this quantity is bounded away from 0 by $\mu$. Calculating,

$$
H_{\bar{z}}(x+i y)=\frac{1}{2}\left(\frac{\partial H}{\partial x}(x+i y)+i \frac{\partial H}{\partial y}(x+i y)\right)<C|y|^{2} .
$$

This implies that

$$
\left|H_{z}(x+i y)\right|=\frac{1}{2}\left|\frac{\partial H}{\partial x}(x+i y)-i \frac{\partial H}{\partial y}(x+i y)\right| \asymp\left|h^{\prime}(x)\right| .
$$

Thus, if $|y|<\eta, H$ is $1+\eta^{2}$-quasiconformal, close to the real line we have that $J(H, x+i y)$ is close to both $\left|H_{z}(x+i y)\right|^{2}$ and $|h(x)|^{2}$. Thus

$$
1+\eta^{2}>\frac{|D H(z)|^{2}}{J(H, z)} \asymp \frac{\left|H_{z}(z)\right|^{2}}{\left|H_{z}(z)\right|^{2}}+2 \frac{\left|H_{z}(z)\right|\left|H_{\bar{z}}(z)\right|}{\left|H_{z}(z)\right|^{2}}+\frac{\left|H_{\bar{z}}(z)\right|^{2}}{\left|H_{z}(z)\right|^{2}}=\left(1+\mu_{H}(z)\right)^{2} .
$$

It immediately follows that

$$
\mu_{H}(z)=O\left(|\operatorname{Im}(z)|^{2}\right) .
$$

Proposition 5.5 ([GSS2], Proposition 2). Let $f: I \rightarrow \mathbb{R}$ be a $C^{3}$ diffeomorphism from a compact interval I with non-empty interior into the real line. There exists $\zeta>0$ and $\delta>0$ such that if $J$ is an interval contained in $I, 0<\alpha<\pi$ and $\operatorname{diam}\left(D_{\alpha}(J)\right)<\delta$, then

$$
f\left(D_{\alpha}(J)\right) \subset D_{\tilde{\alpha}}(f(J)),
$$

where $\tilde{\alpha}=\alpha-\zeta|J| \operatorname{diam} D_{\alpha}(J)$, and $\tilde{\alpha}<\pi$. 
This proposition gives an easy generalization and improvement of the Almost Schwarz Inclusion Principle, Lemma 4.1.

Corollary 5.6 (Almost Schwarz Inclusion in the asymptotically holomorphic case). For every small $a>0$, there exists $\alpha(a)>0$ satisfying $\alpha(a) \rightarrow 0$ and $a / \alpha(a) \rightarrow 0$ as $\alpha \rightarrow 0$, such that the following holds. Let $f: I \rightarrow \mathbb{R}$ be a $C^{3}$ diffeomorphism from a compact interval $I \supset\{0, a\}$ into the real line, with $f(0)=0$ and $f(a)=a$. Let $f$ be a $C^{3}$ extension of $f$ to a complex neighbourhood of $I$, with $f$ asymptotically holomorphic of order 3 on I. Then there exists $\zeta>0$ and $\delta>0$ such that if $\alpha(a)<\alpha<\pi$ and $\operatorname{diam}\left(D_{\alpha}([0, a])\right)<\delta$, then

$$
f\left(D_{\alpha}([0, a])\right) \subset D_{\left(1-\zeta a^{1+\kappa}\right) \alpha}([0, a]),
$$

where $\kappa \in(0,1)$ can be taken arbitrarily close to 1 .

Proof. Fix $\alpha_{0} \in(0, \pi / 4)$. Suppose first that $\alpha<\alpha_{0}<\pi / 2$. Proposition 5.5, we have that

$$
f\left(D_{\alpha}([0, a])\right) \subset D_{\tilde{a}}([0, a]),
$$

where $\tilde{\alpha}=\alpha-\zeta a \operatorname{diam}\left(D_{\alpha}([0, a])\right)=\alpha-\zeta a \frac{a}{\sin \alpha}$. Then since $\alpha$ is small, we have that $\sin \alpha \asymp \alpha$, so that

$$
\tilde{\alpha}=\alpha\left(1-\zeta \frac{a^{2}}{\alpha^{2}}\right)
$$

Suppose that $\delta \in(0,1)$, and let $\alpha(a)=a^{1 / 2-\delta / 2}$. Notice that as $a \rightarrow 0, \alpha(a) \rightarrow 0$ and $a / \alpha(a)=a^{1 / 2+\delta / 2} \rightarrow 0$. Now for any $\alpha>\alpha(a)$, we have that

$$
\tilde{\alpha} \asymp \alpha\left(1-\zeta \frac{a^{2}}{\alpha^{2}}\right) \geq \alpha\left(1-\zeta \frac{a^{2}}{a^{1-\delta}}\right)=\alpha\left(1-\zeta a^{1+\delta}\right) .
$$

Hence

$$
f\left(D_{\alpha}([0, a])\right) \subset D_{\left(1-\zeta a^{1+\delta}\right) \alpha}([0, a]) .
$$

Since this holds for all $\delta \in(0,1)$, we are done.

On the other hand, suppose that $\alpha>\alpha_{0}$, then

$$
\tilde{\alpha}=\alpha-\zeta a \operatorname{diam}\left(D_{\alpha}([0, a])\right) \asymp \alpha-\zeta a^{2}=\alpha\left(1-\zeta \frac{a^{2}}{\alpha}\right) .
$$

Take any $\delta \in(0,1)$ and let $\alpha(a)=a^{\delta}$. Note that as $a \rightarrow 0$ both $\alpha(a)$ and $a / \alpha(a) \rightarrow 0$. Moreover, if $\alpha>\alpha(a)$, then we have

$$
\tilde{\alpha} \asymp \alpha\left(1-\zeta \frac{a^{2}}{\alpha}\right) \geq \alpha\left(1-\zeta a^{2-\delta}\right) .
$$

Hence

$$
f\left(D_{\alpha}([0, a])\right) \subset D_{\left(1-\zeta a^{2-\delta}\right) \alpha}([0, a]) .
$$


Remark. The previous corollary implies that Lemma 4.6 and its corollaries in Sect. 4.1 also hold in the asymptotically holomorphic case if we consider maps $C^{3}$ provided that the Standing Assumptions on p. 26 hold. We will use them in this generality without further comment.

The same argument used to prove Proposition 5.5 can be used to prove:

Corollary 5.7. Let $f: I \rightarrow \mathbb{R}$ be a $C^{3}$ diffeomorphism from a compact interval I with non-empty interior into the real line. There exists $\zeta>0$ and $\delta>0$ such that if $J$ is an interval contained in $I, 0<\alpha<\pi$ and $\operatorname{diam}\left(D_{\alpha}(J)\right)<\delta$, then

$$
f\left(D_{\alpha}(J)\right) \supset D_{\tilde{\alpha}}(f(J)),
$$

where $\tilde{\alpha}=\alpha+\zeta|J| \operatorname{diam} D_{\alpha}(J)$

5.3. $\kappa$-qr box mappings. Let $U$ and $V$ be open Jordan disks in $\mathbb{C}$. We say that a map $F: U \rightarrow V$ is a $\kappa$-qr branched covering if it can be decomposed as $F=G \circ H$ where $H: U \rightarrow U$ is $\kappa$-qc homeomorphism and $G: U \rightarrow V$ is a holomorphic branched covering.

A mapping $F: \mathcal{U} \rightarrow \mathcal{V}$ is qr quasi-box mapping if the following holds. The domain $\mathcal{U}$, is a possibly countable, union of open Jordan disks $U_{i}, \mathcal{V}$ is a union of finitely many open Jordan disks $V_{j}$ with $j=0, \ldots, n-1$ and:

- $V_{0}, \ldots, V_{n-1}$ are pairwise disjoint;

- every connected component $V_{j} \in \mathcal{V}$ is either a connected component of $\mathcal{U}$ or the intersection of $V_{j}$ with $\mathcal{U}$ is a union of $U_{i}$ 's, each of these contained in $V_{j}$ (not necessarily compactly);

- $\quad$ if $U_{i} \subset V_{j}$, then $\bar{U}_{i} \backslash V_{j} \subset \mathbb{R}$.

- $\quad$ For each $i$, there exists $j$ such that $F \mid U_{i}: U_{i} \rightarrow V_{j}$ is a qr branched covering.

A $\kappa$-qr box mapping is defined analogously: we modify the definition of a complex box mapping on p. 2 to only require that on each component $U$ of $\mathcal{U}$ that $F \mid U$ is a $\kappa$ quasiregular covering map onto a component of $\mathcal{V}$. These mappings are $\kappa$-qr branched coverings because of the Stoilow Factorization Theorem, see for example Corollary 5.5.2 in [AIM].

5.4. Additional tools for asymptotically holomorphic maps. To prove complex bounds for asymptotically holomorphic maps, we will need to control their quasiconformal distortion. First, we have the following useful estimate:

Lemma 5.8 (Theorem B [LiS]). Let $f$ be $C^{3}$ with all periodic orbits hyperbolic repelling. Then for any $\alpha$ there exists $\eta=\eta(\alpha)$ such that for any interval $T$ and any $s \in \mathbb{N}$, if $f^{s}: T \rightarrow f^{s}(T)$ is a diffeomorphism, then

$$
\sum_{i=0}^{s}\left|f^{i}(T)\right|^{1+\alpha}<\eta .
$$


In the next lemma, we prove that when we pull back a Poincaré disk $D_{\theta}\left(J_{S}\right)$ by a diffeomorphism, $f^{s}: J_{0} \rightarrow J_{s}$, the total loss of angle is small; so we can estimate the diameters of the pullbacks by the lengths of their real traces. Since the extension of $f$ is asymptotically holomprphic of order three, this will give a bound on the quasiconformal distortion of the extension of $f^{s}$ in terms of $\sum_{j=0}^{s-1}\left|J_{j}\right|^{2}$.

Given an interval $I$, let

$$
\mu(I)=\max _{J}|J|,
$$

where the maximum is taken over all pullbacks $J$ of $I$. Let $\mu^{\prime}(I)=\max _{J}|J|$, where the maximum is taken over all diffeomorphic pullbacks $J$ of $I$. By [K, Lemma 5.2], if $I \cap \omega\left(c_{0}\right) \neq \emptyset$, there exists a constant $\tau_{1} \geq 1$ such that $\mu^{\prime}(I) \leq \tau_{1}|I|$. Consequently, if $I \cap \omega\left(c_{0}\right) \neq \varnothing$, then $\mu(I)$ tends to zero as $|I|$ tends to zero.

If $f$ is persistently recurrent on $c_{0}$ and the Standing Assumptions on p. 26 hold we obtain the following two results.

Lemma 5.9. For any $\theta \in(0, \pi / 2)$, there exist $\varepsilon^{\prime}>0$ and $\tilde{\theta} \in(0, \pi / 2)$ such that for any $\varepsilon \in\left(0, \varepsilon^{\prime}\right)$ the following holds. Let $f$ be an asymptotically holomorphic extension of a $C^{3}$ mapping $f$. Suppose that $\left|J_{S}\right|<\varepsilon, J_{0} \cap \omega\left(c_{0}\right) \neq \varnothing$ and $f^{s}: J_{0} \rightarrow J_{s}$ is a diffeomorphism. Let $\left\{J_{j}\right\}_{j=0}^{s}$ be the chain such that $J_{j}=\operatorname{Comp}_{f^{j}\left(J_{0}\right)} f^{-(s-j)}\left(J_{s}\right)$. Let $U_{s}=D_{\theta}\left(J_{s}\right)$, and set

$$
U_{j}=\operatorname{Comp}_{J_{j}}\left(f^{-(s-j)}\left(U_{s}\right)\right) \text { for } j=0, \ldots, s .
$$

Then $U_{j} \subset D_{\tilde{\theta}}\left(J_{j}\right)$. Moreover, we can make the difference $\theta-\tilde{\theta}$ as small as we like by taking $\varepsilon$ sufficiently small.

Proof. Since $f$ is $C^{3}, f$ has an extension to an asymptotically holomorphic map of order 3 on $M$. Since we are under the Standing Assumptions of Sect. 2, we can apply Lemma 5.8 to find $\eta=\eta(1 / 2)$ such that $\sum_{i=0}^{s}\left|J_{i}\right|^{2}<\eta \max _{0 \leq i \leq k}\left|J_{i}\right|^{1 / 2}$. There exists a constant $\eta_{1}>0$ so that for any $i, 0 \leq i \leq s$, and $\alpha \in(0, \pi / 2)$, we have $\operatorname{diam}\left(D_{\alpha}\left(J_{i}\right)\right) \leq \eta_{1}\left|J_{i}\right| / \sin \alpha$. Let $K$ be equal to the constant $\zeta$ from Proposition 5.5. Let $\tilde{\theta} \in(0, \theta)$ and define

$$
\theta_{s}=\tilde{\theta}+\left(\eta_{1} K / \sin \tilde{\theta}\right) \sum_{i=0}^{s}\left|J_{i}\right|^{2} \leq \tilde{\theta}+\left(\eta_{2} / \sin \tilde{\theta}\right) \max _{0 \leq i \leq k}\left|J_{i}\right|^{1 / 2}
$$

where $\eta_{2}=\eta \eta_{1} K$. We can assume that the difference $\theta-\tilde{\theta}$ is as small as we like. Provided that $\varepsilon>0$ is small enough, we have that $0<\tilde{\theta}<\theta_{s}<\theta$, and that for each $i$, $D_{\tilde{\theta}}\left(J_{i}\right)$ is contained in the domain of the asymptotically holomorphic extension of $f$.

Now, set

$$
\theta_{j}=\theta_{j+1}-K\left|J_{j+1}\right| \operatorname{diam}\left(D_{\theta_{j+1}}\left(J_{j+1}\right)\right) .
$$

We prove that

$$
\theta_{j} \geq \tilde{\theta}+\left(\eta_{1} K / \sin \tilde{\theta}\right) \sum_{i=0}^{j}\left|J_{i}\right|^{2}
$$


for $j=0,1, \ldots, s$ by induction. It holds for $\theta_{s}$ by definition. Suppose that it holds for $\theta_{j+1}$. Since

$$
\theta_{j+1} \geq \tilde{\theta}+\left(\eta_{1} K / \sin \tilde{\theta}\right) \sum_{i=0}^{j+1}\left|J_{i}\right|^{2}
$$

we obviously have that $\theta_{j+1}>\tilde{\theta}$. So that

$$
\begin{aligned}
\theta_{j} & =\theta_{j+1}-K\left|J_{j+1}\right| \operatorname{diam}\left(D_{\theta_{j+1}}\left(J_{j+1}\right)\right) \geq \theta_{j+1}-\eta_{1} K\left|J_{j+1}\right|^{2} / \sin \theta_{j+1} \\
& \geq \tilde{\theta}+\left(\eta_{1} K / \sin \tilde{\theta}\right) \sum_{i=0}^{j+1}\left|J_{i}\right|^{2}-\eta_{1} K\left|J_{j+1}\right|^{2} / \sin \theta_{j+1} \\
& \geq \tilde{\theta}+\left(\eta_{1} K / \sin \tilde{\theta}\right) \sum_{i=0}^{j+1}\left|J_{i}\right|^{2}-\eta_{1} K\left|J_{j+1}\right|^{2} / \sin \tilde{\theta} \\
& \geq \tilde{\theta}+\left(\eta_{1} K / \sin \tilde{\theta}\right) \sum_{i=0}^{j}\left|J_{i}\right|^{2} \geq \tilde{\theta}
\end{aligned}
$$

Remark. As we can see from Lemma 5.9 the loss of angle when we pull back Poincaré disks under asymptotic holomorphic extensions decreases at small scales. In the rest of the paper, the loss of angle will be calculated for intervals of a given size, so the same bounds will hold at smaller scales. This fact will be implicitly assumed from now on.

Lemma 5.10. For any $\theta \in(0, \pi), \delta>0$ and $N \in \mathbb{N}$ there exists $\eta>0$ and $\varepsilon>0$ such that the following holds. Assume that $J_{s} \ni c_{0}$ is a $\delta$-nice real puzzle piece with $\left|J_{S}\right|<\varepsilon$ and that either

(1) $c_{0}$ is even and $J_{s}$ is $\delta$-free or

(2) that $c_{0}$ is odd and all critical points in $\omega\left(c_{0}\right)$ are odd.

Suppose that the chain $\left\{J_{i}\right\}_{i=0}^{s}$ has order bounded by $N$ and $J_{0} \cap \omega\left(c_{0}\right) \neq \emptyset$. Then the asymptotically holomorphic extension

$$
f^{s}: \operatorname{Comp}_{J_{0}} f^{-s}\left(D_{\theta}\left(J_{s}\right)\right) \rightarrow D_{\theta}\left(J_{s}\right)
$$

is $\left(1+\eta\left|\mu\left(J_{S}\right)\right|^{1 / 2}\right)$-quasiregular.

Proof. Let $\mathbf{U}_{s}=D_{\theta}\left(J_{s}\right)$ and set $\mathbf{U}_{j}=\operatorname{Comp}_{J_{j}} f^{-(s-j)}\left(\mathbf{U}_{s}\right)$. Observe that $f^{s} \mid \mathbf{U}_{0}$ is a proper map of bounded degree. Let $k_{1}<s$ be maximal such that $J_{k_{1}}$ contains a critical value of $f$. Notice that if $c_{0}$ is even, there exists a constant $\delta^{\prime}>0$ such that $J_{k_{1}}$ is $\delta^{\prime}$-free. Let $k_{1}^{\prime} \geq k_{1}$ be minimal so that $J_{k_{1}^{\prime}} \subset J_{s}$. Since $f^{s-k_{1}^{\prime}} \mid J_{k_{1}^{\prime}}$ is a diffeomorphism, the sum

$$
\sum_{k=k_{1}^{\prime}}^{s}\left|J_{k}\right|^{2} \leq \eta_{1} \max _{k=k_{1}^{\prime}, \ldots, s-1}\left|J_{k}\right|^{1 / 2}
$$


where $\eta_{1}=\eta_{1}(1 / 2)$ is the constant from Lemma 5.8. Then by Lemma 5.9, there exists a constant $\eta^{\prime}>0$ such that for any point $z_{k} \in \mathbf{U}_{k}, k=k_{1}^{\prime}, k_{1}^{\prime}+1, \ldots, s-1$,

$$
\left|\mu\left(z_{k}\right)\right|=\frac{\bar{\partial} f}{\partial f}\left(z_{k}\right) \leq \eta^{\prime}\left(\operatorname{Im}\left(z_{k}\right)^{2}\right) \leq \eta^{\prime}\left(\operatorname{diam}\left(\mathbf{U}_{k}\right)\right)^{2} \leq \eta^{\prime}\left|J_{k}\right|^{2} .
$$

Where the first inequality follows from the fact that $f$ is asymptotically holomorphic of order three.

So we get that there exists a constant $\eta^{\prime \prime}>0$ such that

$$
\sum_{k=k_{1}^{\prime}}^{s}\left|\mu\left(z_{k}\right)\right| \leq \eta^{\prime \prime} \max _{k=k_{1}^{\prime}, \ldots, s-1}\left|J_{k}\right|^{1 / 2}
$$

Let $s_{1}<k_{1}$ be maximal so that $J_{s_{1}} \subset J_{s}$. The domains $J_{s_{1}}, J_{s_{1}+1}, \ldots, J_{k_{1}^{\prime}}$ are disjoint and small when $J_{k_{1}^{\prime}}$ is small, so, since either all critical points in $\omega\left(c_{0}\right)$ are odd or $c_{0}$ is $\delta^{\prime}$-free by Lemma 4.2, diam $f^{-1}\left(\mathbf{U}_{k_{1}}\right) \asymp\left|J_{k_{1}-1}\right|$. Thus, estimating as before, if $z \in \mathbf{U}_{k_{1}^{\prime}}$,

$$
\sum_{t=s_{1}}^{k_{1}^{\prime}-1}\left|\mu\left(z_{t}\right)\right| \leq \eta^{\prime \prime} \max _{k=s_{1}, s_{1}+1+\cdots k_{1}^{\prime}-1}\left|J_{k}\right|^{1 / 2}
$$

We repeat this argument letting $k_{2}<s_{1}$ be maximal so that $J_{k_{2}}$ contains a critical value of $f$. If no such $k_{2}$ exists, set $k_{2}=0$. Let $k_{2}^{\prime} \geq k_{2}$ be minimal so that $J_{k_{2}^{\prime}} \subset J_{s_{1}}$ and $s_{2}<k_{2}^{\prime}$ maximal so that $J_{s_{2}} \subset J_{s_{1}}$, and if no such $s_{2}$ exists, set $s_{2}=0$. Proceeding inductively, let $p$ be maximal so that $s_{p} \neq 0$.

Let $z \in \mathbf{J}_{0}$ Then

$\sum_{k=0}^{s}\left|\mu\left(f^{k}(z)\right)\right|=\sum_{k=0}^{s_{p}}\left|\mu\left(f^{k}(z)\right)\right|+\sum_{j=1}^{p} \sum_{k=s_{j}}^{s_{j-1}}\left|\mu\left(f^{k}(z)\right)\right| \leq \eta^{\prime \prime} \sum_{j=1}^{p} \max _{k=s_{j}, \ldots s_{j-1}-1}\left|J_{k}\right|^{1 / 2}$.

Since $\max _{k=s_{j}, \ldots s_{j-1}-1}\left|J_{k}\right|^{1 / 2}$ decays exponentially (every $J_{k}$ is $\delta^{\prime}=\delta^{\prime}(\delta, N)>0$ nice), the result follows.

\section{Pulling Back a Poincaré Disc Through High Iterates of First Return Maps}

The results in this section apply to both maps $f \in \mathcal{A}_{\underline{b}}$ and maps $f \in \mathcal{A}_{b}^{3}$ for which the asymptotically holomorphic extensions are as in Sect. 5.2. Recall, we will always work under the Standing Assumptions on p. 26.

\subsection{Pullbacks through a monotone branch.}

Proposition 6.1. For each $\delta>0$ and $C>0$ there exist $\delta^{\prime}>0$ and $\lambda^{\prime} \in(0,1)$, and for each $\theta \in(0, \pi)$ there exists $\varepsilon>0$ such that the following holds. Let $I^{0}$ be an interval with $\left|I^{0}\right|<\varepsilon$ and let $I^{1} \subset I^{0}$ be a first return domain to $I^{0}$. Let $r$ be so that $\left.R_{I^{0}}\right|_{I^{1}}=f^{r}$. Assume $\left.f^{r}\right|_{I^{1}}$ is monotone and can be decomposed into at most $b$ maps 
of the form $g \circ p_{\ell} \circ h$, where $p_{\ell}=x^{\ell}$ for some odd integer $\ell>0$ and $g$ and $h$ are diffeomorphisms with bounded distortion. Assume

$$
\left|f^{r}(c)-c\right| \geq \delta\left|I^{1}\right| \text { and }\left|D f^{r}(x)\right| \leq C
$$

for all critical points $c$ of $\left.f^{r}\right|_{I^{1}}$ and all $x \in I^{1}$. Let $\left\{G_{j}\right\}_{j=0}^{p r}$ be a disjoint chain with $\left(\left.f^{r}\right|_{I^{1}}\right)\left(G_{j r}\right)=G_{(j+1) r}$ for $j=0 \ldots p-1, G_{p r} \subset I^{0}$ and $G_{0} \cap \omega\left(c_{0}\right) \neq \emptyset$. Assume there exist disjoint intervals $K, F^{1}, F^{2} \subset I^{0}$ with the following properties.

- $G_{p r} \subset K$,

- $F^{1}$ and $F^{2}$ are contained in fundamental domains for $\left.f^{r}\right|_{I^{1}}$

- $\left|F^{i}\right|>\delta\left|I^{0}\right|$ for $i=1,2$, and

- $K$ is contained in the convex hull of $F^{1}$ and $F^{2}$.

Let

$$
V=D_{\theta}(K) \cap \mathbb{C}_{G_{p r}} \text { and } U_{i}=\operatorname{Comp}_{G_{i}} f^{-(p r-i)}(V), \text { for } i=0, \ldots, p r .
$$

Then for each $z \in U_{0}$ there exists an interval $K^{\prime}$ such that

$$
z \in D_{\lambda^{\prime} \theta}\left(K^{\prime}\right),
$$

where $G_{0} \subset K^{\prime} \subset\left(1+2 \delta^{\prime}\right) K^{\prime} \subset I^{1}$.

Proof. Without loss of generality we will assume the map $\left.f^{r}\right|_{I^{1}}$ is orientation preserving and that its fixed point $p$ is equal to 0 . The orientation reversing case will follow from the orientation preserving case by considering by $f^{2 r}$ instead of $f^{r}$ and applying Lemma 4.6 once.

Since $\left.f^{r}\right|_{I^{1}}$ has no periodic attractors we have that for each of its critical points $c$ there exists a maximal $m(c)>0$ so that $f^{r j}(c) \in I^{1}$ of all $0 \leq j \leq m(c)$. Consider $\Omega=\cup\left\{c, \ldots, f^{m(c)}(c)\right\}$, where the union is taken over all critical points of $\left.f^{r}\right|_{I^{1}}$. Since each $F^{k}$ is contained in a fundamental domain, $F^{k} \cap \Omega$ contains at most $b$ points for $k=1,2$. So by slightly shrinking $F^{k}$, if necessary, we can assume $\Omega \cap F^{k}=\emptyset$ and that the convex hull of $F^{1}$ and $F^{2}$ is $\delta / 2$-well inside $I^{0}$. Observe this means that no pullback of $F^{k}$ under $\left.f^{r}\right|_{I^{1}}$ contains a critical point for $k=1,2$.

We will first prove the proposition for the unicritical case. Unless is specify otherwise, we will assume that all pullbacks of Poincaré domains under $f$ are taken under the branch of $f^{-1}$ that maps $G_{i+1}$ to $G_{i}$ for $i \in\{0, \ldots p r-1\}$. Observe that the inverse branches of $f$ extend to the complex plane and we are able to express which inverse branch we are using by choosing the domain : when we write $f^{-1}: \mathbb{C}_{G_{j+1}} \rightarrow \mathbb{C}$, we always mean the choice of inverse branch that maps $G_{j+1}$ to $G_{j}$. Consider the chain $\left\{H_{j}\right\}_{j=0}^{r}$, with $H_{r}=I^{0}$ and $H_{0}=I^{1}$.

Let $c$ be the critical point of $\left.f^{r}\right|_{I^{1}}$. We will denote by $c_{j}=f^{j}(c)$ for $j \in \mathbb{Z}$, where the inverse images of $c$ will be taken under the pullbacks defined above. Since $c$ is a critical point of $f^{r}$, there exists $i$ (maximal) so that $0 \leq i<r$ and $c_{i}=c^{\prime}$ for $c^{\prime} \in \operatorname{Crit}(f)$. To orient ourselves we will assume $c>0$. From the fact that $f^{r}$ has no periodic attractors, and that $f^{r}(0)=0$, we have that $0<c_{-r}<c<c_{r}$. The case $c<0$ is analogous to this one, taking into consideration that in this case $c_{r}<c<c_{-r}<0$.

We can assume that $c_{r}$ is not contained in $G_{p r}$. Otherwise, let $J_{r}$ be the convex hull of $F^{1}$ and $F^{2}$. Since $J_{r} \backslash(1+\delta)^{-1} J_{r}$ is disjoint from $\Omega$, we can apply Corollary 4.7 to find $\hat{\lambda}>0$ so that $U_{(p-1) r} \subset D_{\hat{\lambda} \theta}\left(J_{0}\right) \cap \mathbb{C}_{G_{(p-1) r}}$, where $f^{r}\left(J_{0}\right)=J_{r}$. Since $G_{(p-1) r}$ does not contain $c_{r}$ we can start the proof at time $(p-1) r$, instead of $p r$. 
We will first consider the case when $G_{p r}$ is in the right hand component of $\mathbb{R} \backslash\left\{c_{r}\right\}$, $G_{0}$ is in the left one, and $F^{1}$ and $F^{2}$ are in opposite components. Without loss of generality assume $F^{2}$ and $G_{p r}$ are on the same component of $\mathbb{R} \backslash\left\{c_{r}\right\}$. Note that the relative position of $F^{1}$ and $F^{2}$ with respect to 0 has not been specified; see Fig. 6 for a possible configuration.

Let $R_{p r}=F^{2}$ and $L_{p r}=F^{1}$ and consider the chain $\left\{R_{j}\right\}_{j=(p-1) r}^{p r}$ given by the pullbacks under the branches of $f^{-1}$ which map $G_{j+1}$ to $G_{j}$. Observe that all intervals $R_{j}$ are contained in $\mathbb{R}$ for $(p-1) r \leq j \leq p r$. On the other hand, if we consider the pullbacks of $L_{p r}$ under the same maps, we get that $L_{(p-1) r+i+1}$ is not in the same component of $\mathbb{R} \backslash\left\{c^{\prime}\right\}$ as $G_{(p-1) r+i+1}$ (see Fig. 7).

This means that the pullback of $L_{(p-1) r+i+1}$ under the branch of $f^{-1}$ which maps $G_{(p-1) r+i+1}$ to $G_{(p-1) r+i}$ does not lie in the real line.

By Lemma 4.5 , there exists $\lambda \in(0,1)$ so that

$$
V \subset D_{\lambda \theta}\left(L_{p r}\right) \cup D_{\lambda \theta}\left(R_{p r}\right) \cup A,
$$

where $A$ is the bounded component of the complement of $D_{\lambda \theta}\left(L_{p r}\right) \cup D_{\lambda \theta}\left(R_{p r}\right)$. In order to control the pullbacks of points in $V$ we will, separately, control the pullbacks of points in $D_{\lambda \theta}\left(L_{p r}\right), D_{\lambda \theta}\left(R_{p r}\right)$ and $A$.

Let $q$ be maximal with $0 \leq q<p$ and so that $G_{q r}$ and $G_{p r}$ are in the same component of $\mathbb{R} \backslash\left\{c_{r}\right\}$. From the choice of $q$, the intervals $R_{j r}$ and $G_{j r}$ are in the same component of $\mathbb{R} \backslash\left\{c_{r}\right\}$ for $j=q, \ldots, p$.

To pullback $D_{\lambda \theta}\left(R_{p r}\right)$. Let $z^{p r} \in D_{\lambda \theta}\left(R_{p r}\right)$ and consider the sequence of points $\left\{z^{j}\right\}_{j=0}^{p r}$, where $z^{j}$ is preimage of $z^{j+1}$ under the branch of $f^{-1}$ specified above.

(a) Assume that $q=0$ or that $q>0$ and the intervals $G_{(q-1) r}$ and $R_{(q-1) r}$ lie in the same component of $\mathbb{R} \backslash\left\{c_{r}\right\}$. Then, since the chain $\left\{R_{j}\right\}_{j=(q-1) r}^{p r}$ is disjoint we can apply Lemma 5.9 to find $\lambda_{0} \in(0,1)$ so that

$$
z^{0} \in D_{\lambda_{0} \theta}\left(R_{0}\right) \text {. }
$$

Observe that $R_{0}$ lies between 0 and $R_{p r}$ and that the distance between $R_{0}$ and 0 is comparable to $\left|R_{0}\right|$. So there exists $\delta_{0}>0$ so that $\left(1+2 \delta_{0}\right) R_{0} \subset I^{1}$, and the proposition follows.

(b) Assume that $q>0$ and the intervals $G_{(q-1) r}$ and $R_{(q-1) r}$ lie in opposite components of $\mathbb{R} \backslash\left\{c_{r}\right\}$. Apply Lemma 5.9 to find $\lambda_{1} \in(0,1)$ so that



Fig. 6. Configuration w.r.t. $c_{r}$ (the critical value of $f^{r}$ )

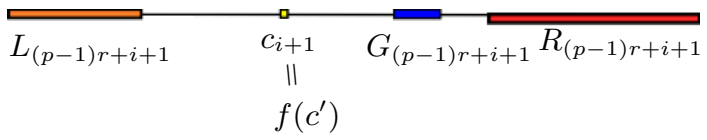

Fig. 7. Pullback at time $i+1$ 
If $q=1$ then $(q-1) r=0$ and the result follows as in (a). If $q>1$, then by Lemma 5.9, we can choose $\lambda_{2}>0$ so that

$$
z^{(q-2) r+i+1} \in D_{\lambda_{2} \theta}\left(R_{(q-2) r+i+1}\right) .
$$

Observe that $R_{(q-2) r+i+1}$ and $G_{(q-2) r+i+1}$ are in different components of $\mathbb{R} \backslash\left\{c_{i+1}\right\}$. By the assumptions on $f^{r}$, we know that the interval $\left(c_{-r-j}, c_{j}\right)$ has size comparable to $\left|H_{j}\right|$ for $j \in\{i, i+1\}$. So we can apply Lemma 4.4 to find $\lambda_{3}>0$ so that image of $D_{\lambda_{2} \theta}\left(R_{(q-2) r+i+1}\right)$ under the branch that maps $G_{(q-2) r+r+i}$ to $G_{(q-2) r+r+i-1}$ is contained in $D_{\lambda_{3} \theta}\left(c_{-r-i}, c_{i}\right)$. See Fig. 8 .

In particular

$$
z^{(q-2) r+i} \in D_{\lambda_{3} \theta}\left(c_{-r-i}, c_{i}\right)
$$

Finally, if we let $R_{(q-2) r+i}^{\prime}=\left(c_{-r-i}, c_{i}\right)$ and we consider the chain $\left\{R_{j}^{\prime}\right\}_{j=0}^{(q-2) r+i}$ the result follows as in Case (a).

In conclusion, exist an interval $\tilde{K} \subset I^{1}$ and constants $\tilde{\lambda} \in(0,1)$ and $\tilde{\delta}>0$ so that $(1+2 \tilde{\delta}) \tilde{K} \subset I^{1}$ and for all $z^{p r} \in D_{\tilde{\lambda} \theta}\left(R_{p r}\right)$ we have that

$$
z^{0} \in D_{\tilde{\lambda} \theta}(\tilde{K}) .
$$

To pullback $D_{\lambda \theta}\left(L_{p r}\right)$. Pick $z^{p r} \in D_{\lambda \theta}\left(L_{p r}\right)$ and consider the sequence of points $\left\{z^{j}\right\}_{j=0}^{p r}$, where $z^{j}$ is the preimage of $z^{j+1}$ under the branch of $f^{-1}$ specified above.

By Lemma 5.9, there exists $\lambda_{1}^{\prime}>0$ so that

$$
z^{(p-1) r+i+1} \in D_{\lambda_{1}^{\prime} \theta}\left(L_{(p-1) r+i+1}\right) .
$$

By assumption $L_{(p-1) r+i+1}$ and $G_{(p-1) r+i+1}$ are in different components of $\mathbb{R} \backslash\left\{c_{i+1}\right\}$. Let $\hat{L}=\left(f^{-1} \mid \mathbb{C}_{G_{i+1}}\right)\left(D_{\lambda_{1}^{\prime} \theta}\left(L_{(p-1) r+i+1}\right)\right)$. By Lemma 4.4 , there exists $\lambda_{2}^{\prime}>0$ so that

$$
\hat{L} \subset D_{\theta}\left(c_{i}, w_{i}\right) \cup D_{\lambda_{2}^{\prime} \theta}\left(R_{(p-1) r+i}\right) \cup A^{\prime}
$$

where $A^{\prime}$ is the bounded component of the complement of $D_{\theta}\left(c_{i}, w_{i}\right) \cup D_{\lambda_{2}^{\prime} \theta}\left(R_{(p-1) r+i}\right)$ and $w_{i}$ is defined as follows. If $c_{r} \in I^{1}$, let $w_{i}=c_{2 r-i}$. If $c_{r} \notin I^{1}$, let $w_{i}$ be the boundary

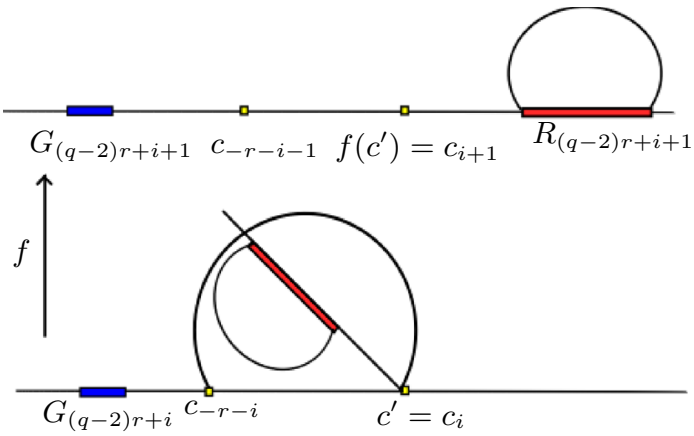

Fig. 8. Controlling the loss of angle after $f\left(c^{\prime}\right)\left(c^{\prime} \in \operatorname{Crit}(f)\right)$ 
point of $H_{i}$ that lies on the same side of $c_{i}$ as $G_{(p-1) r+i}$. In either case, the size of the interval $\left(c_{i}, w_{i}\right)$ is comparable to $\left|R_{(p-1) r+i}\right|$ and $\left|H_{i}\right|$. See Fig. 9.

Consider the chain $\left\{B_{j}\right\}_{j=0}^{r-1}$ with $B_{r-1}=\left(c_{i}, w_{i}\right)$ and $B_{0}$ the pullback of $B_{r-1}$ contained in $H_{i+1}$. By Lemma 5.9, we can choose $\lambda_{3}^{\prime}>0$ so that

$$
\operatorname{Comp}_{B_{0}}\left(f^{-(r-1)} D_{\theta}\left(B_{r-1}\right)\right) \subset D_{\lambda_{3}^{\prime} \theta}\left(B_{0}\right) .
$$

By Lemma 4.4, we can choose $\lambda_{4}^{\prime}>0$ so that the component of the inverse image of $D_{\lambda_{3}^{\prime} \theta}\left(B_{0}\right)$ under $f$ that lies on the same side of $c_{i}$ as $G_{(p-1) r+i}$, denoted by $\hat{B}$, satisfies the following

$$
\hat{B} \subset D_{\theta}\left(c_{i}, w_{i}\right) \cup D_{\lambda_{4}^{\prime} \theta}\left(R_{(p-1) r+i}\right) \cup C^{\prime}
$$

where $C^{\prime}$ is the bounded component of the complement to $D_{\theta}\left(c_{i}, w_{i}\right) \cup D_{\lambda_{4}^{\prime} \theta}\left(R_{(p-1) r+i}\right)$. See Fig. 10.

Thus,

$$
z^{(p-1) r+i} \in D_{\theta}\left(c_{i}, w_{i}\right) \cup D_{\lambda_{4}^{\prime} \theta}\left(R_{(p-1) r+i}\right) \cup C^{\prime} .
$$

By definition of $\lambda_{4}^{\prime}$ we have that one of the following holds:

(i) $z^{k r+i} \in D_{\theta}\left(c_{i}, w_{i}\right)$ for all $k \in\{q-1, \ldots p-1\}$. In particular, $z^{(q-1) r+i} \in$ $D_{\theta}\left(c_{i}, w_{i}\right)$. If we let $R_{(q-1) r+i}^{\prime}=\left(c_{i}, w_{i}\right)$, the proposition follows as in (a); considering the chain $\left\{R_{j}^{\prime}\right\}_{j=0}^{(q-1) r+i}$, instead of the chain $\left\{R_{j}\right\}_{j=0}^{(q-1) r+i}$.

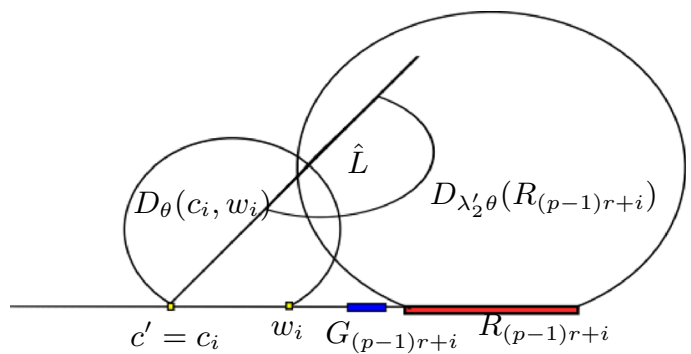

Fig. 9. Use two domains to control the loss of angle at $c_{i}=c^{\prime} \in \operatorname{Crit}(f)$

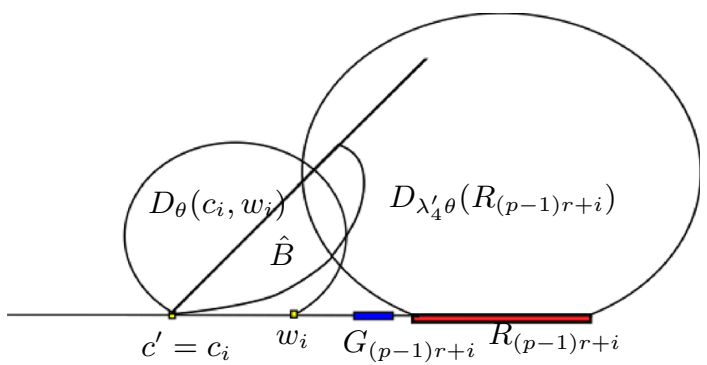

Fig. 10. Adjust the angle at $c_{i}=c^{\prime} \in \operatorname{Crit}(f)$ 
(ii) There exists $k \in\{q-1, \ldots p-1\}$ so that $z^{k r+i} \in D_{\lambda_{4} \theta}\left(R_{(p-1) r+i}\right)$. Then define $R_{k r+i}^{\prime}=R_{(p-1) r+i}$ and apply arguments (a) or (b) above.

(iii) $z^{k r+i} \in C^{\prime}$ for all $k \in\{q-2, \ldots p-1\}$. In particular, $z^{(q-2) r+i} \in C^{\prime}$. From Cases (i) and (ii) we know that there exist $\delta_{5}^{\prime}>0, \lambda_{5}^{\prime} \in(0,1)$ and intervals $K_{1}$ and $K_{2}$ which are $\delta_{5}^{\prime}$-well inside $I^{1}$ so that the following holds. If $z^{(q-2) r+i} \in D_{\theta}\left(c_{i}, w_{i}\right)$ then $z^{0} \in D_{\lambda_{5}^{\prime} \theta}\left(K_{1}\right)$. And if $z^{(q-2) r+i} \in D_{\lambda_{4}^{\prime} \theta}\left(R_{(p-1) r+i}\right)$ then $z^{0} \in D_{\lambda_{5}^{\prime} \theta}\left(K_{2}\right)$. So by the continuity of $f$, if $z^{(q-2)+i} \in C^{\prime}$ we have that $z^{0}$ is contained in the bounded component of the complement of $D_{\lambda_{5}^{\prime} \theta}\left(K_{1}\right) \cup D_{\lambda_{5}^{\prime} \theta}\left(K_{2}\right)$. If we let $K_{3}$ denote the convex hull of $K_{1}$ and $K_{2}$ we have that $z^{0} \in D_{\lambda_{5}^{\prime} \theta}\left(K_{3}\right)$. Finally, the existence of $\lambda_{5}^{\prime}$ implies that $\left|K_{1}\right|$ is comparable to $\left|K_{3}\right|$. Since $K_{1}$ is a fundamental domain, or contained in one, the distance between $K_{1}$ and 0 is comparable to $\left|K_{1}\right|$. So there exists $\delta_{6}^{\prime}>0$ so that $K_{3}$ is $\delta_{6}^{\prime}$-well inside $I^{1}$, and the proposition follows.

To pullback $A$. Making $\lambda_{4}^{\prime}$ smaller, if necessary, we can make sure that the following holds. Given $z^{p r} \in D_{\lambda \theta}\left(R_{p r}\right)$ then $z^{(p-1) r+i} \in D_{\lambda_{4}^{\prime} \theta}\left(R_{(p-1) r+i}\right)$. Observe this implies that given $z^{p r} \in A$ then

$$
z^{(p-1) r+i} \in D_{\theta}\left(c_{i}, w_{i}\right) \cup D_{\lambda_{4}^{\prime} \theta}\left(R_{(p-1) r+i}\right) \cup C^{\prime},
$$

and the proposition follows from one of the previous cases.

This completes the proof of the proposition in this case. The arguments given in this proof also deal with the case when $G_{p r}$ and $G_{0}$ lie on the same side of $c_{r}$ and when $F^{1}$ and $F^{2}$ lie on the same side of 0 . Thus the unicritical case follows.

If $\left.f^{r}\right|_{I^{1}}$ is multicritical it will have at most $b$ critical points. It is clear that, by the monotonicity of $\left.f^{r}\right|_{I^{1}}$, the proposition in this case follows by applying the unicritical case at most $b+1$ times.

Remark 1. From the proof of Proposition 6.1 we get the extra information that $K \subset[x, y]$ where $x, y \in I^{0}$ are of one the two following types:

- $x \in\left\{c_{-r}^{j}, c^{j}, c_{r}^{j}\right\}$, for $j \in\{1, m\}$ and $y \in \partial I^{0}$;

- $x \in\left\{c_{-r}^{j}, c^{j}, c_{r}^{j}\right\}$ and $y \in\left\{c_{-r}^{j+1}, c^{j+1}, c_{r}^{j+1}\right\}$, for $j \in\{1, \ldots m-1\}$.

Remark 2. We cannot take real pullbacks of the fundamental domains at each step when there are critical points since we cannot control the order of the pullback of the larger interval $\left(F^{1}, F^{2}\right)$.

Remark 3. If $f^{r}$ is a diffeomorphism, we do not require the bound on the derivative.

\subsection{Pullbacks for which the modality is bounded.}

Proposition 6.2. For any $\delta>0$ and $N \in \mathbb{N}$ there exists $\lambda>0$, and for any $\theta \in(0, \pi)$ there exists $\varepsilon>0$ so that the following holds. Let I be a nice interval with $|I|<\varepsilon$. Suppose that either:

(1) $I$ is $\delta$-free or

(2) $c_{0}$ is odd.

Let $J$ be an $N$-modal pullback of $I$ by $f^{t}$ for some $t>0$ with $J \cap \omega\left(c_{0}\right) \neq \emptyset$. Let $V=D_{\theta}(I)$ and $U_{i}:=\operatorname{Comp}_{f^{i}(J)} f^{-(t-i)}(V)$. Then $U_{0} \subset D_{\lambda \theta}(J)$. 
Proof. If we are in case (1), then we have the following. Let $\left\{G_{j}\right\}_{j=0}^{t}$ be the chain with $G_{t}=I$ and $G_{0}=J$. Since $I$ is $\delta$-internally free, $F^{\prime} \subset I$ is a nice pair, $\omega\left(c_{0}\right) \cap I \subset F^{\prime}$ and $F^{\prime} \subset(1+2 \delta)^{-1} I$. Let $\left\{H_{j}\right\}_{j=0}^{t}$ be the chain corresponding to the pullbacks of $F^{\prime}$, with $H_{j} \subset G_{j}$. Let $F_{j}^{ \pm}$be the components of $G_{j} \backslash H_{j}$. Observe that $G_{t}, H_{t}$ is a nice pair, so their pullbacks are also nice pairs. Since each of the intervals $G_{j}$ intersects $\omega\left(c_{0}\right)$ whereas $G_{j} \cap H_{j}$ does not intersect $\omega\left(c_{0}\right)$ it follows that the intervals $F_{j}^{ \pm}$are all disjoint. Moreover, since $H_{j} \subset\left(1+2 \delta^{\prime}\right)^{-1} G_{j}$, it follows that the intervals $F_{j}^{+}$and $F_{j}^{-}$are not small compared to $G_{j}$.

Decompose $f^{t}: J \rightarrow I$ as $f^{t} \mid J=D_{N+1} \circ P_{\ell_{N}} \circ \cdots \circ P_{\ell_{2}} \circ D_{2} \circ P_{\ell_{1}} \circ D_{1}$, where each $D_{i}$ is a diffeomorphism and $P_{\ell_{i}}: z \mapsto z^{\ell_{i}}$. The result follows by using Lemma 5.9 to control the loss of angle when we pull back by a $D_{i}$ and Lemma 4.2 when we pull back by a polynomial. The first paragraph of this proof ensures that whenever we pullback by an even polynomial, the critical value is not close to the boundary.

6.3. Pulling back through a non-monotone branch. In this subsection we will control pullbacks of Poincaré disks through a long cascade of central returns in the principal nest.

Let $I$ be a nice interval containing a critical point $c \in \omega\left(c_{0}\right)$. Recall the definition of the principal nest: $I^{0}=I$ and if $I^{n}$ is defined, we set $I^{n+1}=\mathcal{L}_{c}\left(I^{n}\right)$ for $n \in \mathbb{N}$. Let $r$ be so that $\left.R_{I}\right|_{I^{1}}=f^{r}$. If $\left.f^{r}\right|_{I^{1}}$ has a turning point, define $\tilde{m}$ to be minimal with the property that there is a turning point $c^{\prime}$ of $\left.f^{r}\right|_{I^{1}}$ such that $f^{r}\left(c^{\prime}\right) \notin I^{\tilde{m}}$. Note that if $I$ is periodic, $I=I^{1}=I^{2}=\cdots$ and $\tilde{m}=\infty$. In this case $f^{r}\left(I^{\tilde{m}}\right) \subset I^{\tilde{m}}$ and $f^{r}\left(\partial I^{\tilde{m}}\right) \subset \partial I^{\tilde{m}}$, so $f$ is renormalizable and $I^{\tilde{m}}=I^{\infty}$ is periodic. Under the above assumptions we have the following.

Proposition 6.3 (cf. [KSvS], Lemma 11.1). There exists a constant $\hat{\rho}>0$ and for each $\delta>0$ there exist $\delta^{\prime}>0$ and $\lambda \in(0,1)$ so that for each $\theta \in(0, \pi)$ there exists $\varepsilon>0$ with the following properties. Let $I^{0}=I, I^{1}=\mathcal{L}_{c}(I)$ and assume that $|I|<\varepsilon$ and $(1+2 \delta) I^{1} \subset I$. Let $r$ be so that $\left.R_{I^{0}}\right|_{I^{1}}=f^{r}$. Additionally, if $c$ is odd assume $\left.f^{r}\right|_{I^{1}}$ is monotone. Let $\left\{G_{j}\right\}_{j=0}^{p r}$ be a disjoint chain with $G_{j r} \subset I^{1}$ for all $0 \leq j \leq p-1$, so that pullbacks of $G_{p r}$ and $I$ are nested or disjoint. Let $\left\{\hat{G}_{j}\right\}_{j=0}^{p r}$ be a chain with $G_{p r} \subset \hat{G}_{p r} \subset(1+2 \delta) \hat{G}_{p r} \subset I$ and $G_{0} \subset \hat{G}_{0}$ and define

$$
V=D_{\theta}\left(\hat{G}_{p r}\right) \cap \mathbb{C}_{G_{p r}} \text { and } \quad U=\operatorname{Comp}_{G_{0}} f^{-p r}(V) .
$$

If $c$ is even, then for each $z \in U$, there exists an interval $K$ such that either

$$
z \in D_{\lambda \theta}(K) \text { and } G_{0} \subset K \subset\left(1+2 \delta^{\prime}\right) K \subset I
$$

or there exists $0 \leq p^{\prime} \leq p$ and intervals $K \subset I^{\prime} \subset I^{\tilde{m}}$ with

$$
f^{p^{\prime} r}(z) \in D_{\lambda \theta}(K) \text { and } G_{p^{\prime} r} \subset K \subset\left(1+2 \delta^{\prime}\right) K \subset I^{\prime}
$$

where $I^{\prime}$ is $\hat{\rho}$-nice if $\tilde{m}<\infty$.

If $c$ is odd, then for each $z \in U$, there exists an interval $K$ such that (6.1) holds. 
Proof. Let $z \in U$. If $p \leq 10$ or if $\tilde{m}=1$, then we can apply Corollary 4.8 finitely many times to complete the proof. If $p \leq 10$, then (6.1) holds and if $\tilde{m}=1$, then (6.2) holds for $p^{\prime}=p-1$, so we will assume that $p>10$ and $\tilde{m} \geq 2$.

Let us first assume that the $R_{I}$ restricted to $I^{1}$ is monotone. Apply Corollary 4.8 twice to find $\lambda_{1}>0$ so that $f^{(p-2) r}(z) \subset D_{\lambda_{1} \theta}\left(I^{2}\right)$. If the size of $I^{2}$ is comparable to the size of $I^{1}$ we can apply Lemma 3.10 to $\left.f^{r}\right|_{I^{2}}$. Since $(1+2 \delta) I^{1} \subset I^{0}$ there exists $\delta_{1}>0$ so that $I^{2}$ is $\delta_{1}$ well-inside $I^{1}$. Using the connected components of $I^{1} \backslash\left(1+\delta_{1}\right) I^{2}$ we can find suitable fundamental domains and apply Proposition 6.1 to show (6.1).

If $\left|I^{2}\right| /\left|I^{1}\right|<\varepsilon$, for $\epsilon>0$ small, we get that $I^{2}$ is deep-inside $J=(1+\delta) I^{1}$. Since $J$ is well-inside $I$ and $f^{r}$ is monotone there exists $C=C(\varepsilon)>0$ so that $\hat{J}=\operatorname{Comp}_{c_{0}} f^{-r}(J) \subset(1+2 C) \hat{J} \subset J$. Note that $C \rightarrow \infty$ as $\epsilon \rightarrow 0$. Since $\tilde{m}>1$, there are no critical points or critical values of $f^{r} \mid I^{1}$ contained in $I \backslash I^{1}$, so we can apply Lemma 4.6 to find $\lambda_{2} \in(0,1)$ so that $\operatorname{Comp}_{c} f^{-r} D_{\lambda_{1} \theta} \subset D_{\lambda_{2} \theta}\left(J^{\prime}\right)$. Making $\epsilon$ small enough we get the following. Given $x \in \hat{J}$

$$
\operatorname{Comp}_{c} f^{-r} D_{\lambda_{1} \theta}(J) \subset D_{\lambda_{2} \theta}\left(J^{\prime}\right) \subset D_{\lambda_{1} \theta}(J),
$$

so we get (6.1).

From now on we will assume that $c$ is even.

Claim 1. There exists a (universal) constant $v \in(0,1)$ such that if $\left|I^{2}\right| /\left|I^{0}\right| \leq v$ then (6.1) holds.

Proof of Claim 1. Assume $\left|I^{2}\right| /\left|I^{0}\right| \leq v$. Since $c$ is even, there exists $C=C(v)$ so that $(1+2 C) I^{2} \subset I^{0}$, which in turn implies that there exists a constant $C^{\prime}=C^{\prime}(v)$ so that $\left(1+2 C^{\prime}\right) I^{2} \subset I^{1}$. Recall that $f^{(p-2) r}(z) \in D_{\lambda_{1} \theta}\left(I^{2}\right)$. By Corollary 4.8, there exists $\lambda_{3} \in(0,1)$ such that for each $x \in I^{2}$,

$$
\operatorname{Comp}_{x} f^{-r}\left(D_{\lambda_{1} \theta}\left(0.5 I^{1}\right)\right) \subset D_{\lambda_{3} \theta}\left(I^{2}\right) \text {. }
$$

If $v$ is sufficiently small, depending only on the choice of 0.5 , then

$$
D_{\lambda_{3} \theta}\left(I^{2}\right) \subset D_{\lambda_{1} \theta}\left(0.5 I^{1}\right)
$$

from which it follows that (6.1) holds.

From now on, we will assume that $\left|I^{2}\right| /\left|I^{0}\right|>v$.

Let $E_{1}$ and $E_{2}$ be the outer monotone branches of $f^{r} \mid I^{1}$. In order to be definite, let $E_{1}$ be so that $f^{r} \mid E_{1}$ is orientation preserving. Let $c_{l}$ be the turning point in $\partial E_{1}$ and $c_{r}$ the turning point in $\partial E_{2}$

Claim 2. There exist universal constants $\kappa_{1}, \kappa_{2}>0$ and $C>1$ such that

(1) for any critical point $c$ of $f^{r}\left|I^{2}, \operatorname{dist}\left(f^{r}(c), c\right) \geq \kappa_{1}\right| I^{0} \mid$;

(2) $\left|\left(f^{r}\right)^{\prime}(x)\right| \leq C$ for any $x \in I^{2}$;

(3) either $f^{r}\left(I^{\tilde{m}}\right) \cap I^{\tilde{m}}=\emptyset$ or $\left|E_{i} \cap I^{\tilde{m}}\right| \geq \kappa_{2}\left|I^{0}\right|$, for $i=1,2$.

Proof of Claim 2. From Claim 1 we have that $\left|I^{2}\right| /\left|I^{0}\right|>v$, so (1) and (2) follow from Lemma 3.10. Statement (3) follows from Statements (1) and (2). $\checkmark$

Claim 3. It is enough to prove the proposition for the case that $G_{p r} \subset I^{\tilde{m}}$ and $f^{p r}(z) \in$ $D_{\hat{\lambda} \theta}(J) \backslash D_{\theta}\left(I^{1}\right)$ for some constant $\hat{\lambda} \in(0,1)$ and $\hat{\delta}>0$ (which do not depend on $\theta)$ and some interval $I^{2} \subset J \subset(1+2 \hat{\delta}) J \subset I^{1}$. In particular, we can assume that $f^{r}\left(I^{\tilde{m}}\right) \cap I^{\tilde{m}} \neq \varnothing$ (because otherwise (6.1) holds). 
Proof of Claim 3. If necessary we can apply Corollary 4.8 to obtain $\lambda_{4} \in(0,1)$ so that $f^{(p-1) r}(z) \in D_{\lambda_{4} \theta}\left(I^{1}\right)$. Replacing $p$ by $p-1$ and $\theta$ by $\lambda_{4} \theta$ we may assume that $\hat{G}_{p r} \subset I^{1}$ and $f^{p r}(z) \in D_{\theta}\left(I^{1}\right)$. If $z \in D_{\theta}\left(I^{1}\right)$, then (6.1) holds and the proof is completed. So we may assume that there exists a maximal $q$ with $0 \leq q<p$ such that $f^{q r}(z) \notin D_{\theta}\left(I^{1}\right)$. Since $f^{(q+1) r}(z) \in D_{\theta}\left(I^{1}\right)$, by Corollary 4.8 there exist $\delta_{2}>0$ and $\lambda_{5} \in(0,1)$ such that

$$
f^{q r}(z) \in D_{\lambda_{5} \theta}(J) \backslash D_{\theta}\left(I^{1}\right),
$$

where $J$ is an interval with $I^{2} \subset J \subset\left(1+2 \delta_{2}\right) J \subset I^{1}$ (see Fig. 11).

Let us first see what happens if $f^{r}\left(I^{\tilde{m}}\right) \cap I^{\tilde{m}}=\emptyset$. In this case the only way that an interval $G_{j}$ could have a pullback under $f^{r}$ is if it is a pullback under $E_{1}$. Assume this is the case. Claim 1 and Lemma 4.5 allow us to find $\delta_{3}, \lambda_{6}>0$ and $K$ an interval $\delta_{3}$-well-inside $E_{1}$ such that $f^{q r}(z) \in D_{\lambda_{6} \theta}(K)$. We can find fundamental domains of size comparable to $I^{1}$ for the map $f^{r} \mid E_{1}: E_{1} \rightarrow f^{r}\left(E_{1}\right)$ as follows. On one side the fundamental domain is given by an interval in $I^{0} \backslash I^{1}$ and on the other side it is given by the $c_{l}$ and $f^{r}\left(c_{l}\right)$. Applying Proposition 6.1 we get (6.1). From now on assume $f^{r}\left(I^{\tilde{m}}\right) \cap I^{\tilde{m}} \neq \varnothing$.

If $q \leq 2$, then by applying Corollary 4.8 at most twice, we obtain (6.1). So we may assume that $q \geq 3$. If $G_{q r} \subset I^{\tilde{m}}$, then the claim follows from (6.3). So we will assume that $G_{q r} \not \subset I^{\tilde{m}}$. From this assumption and the fact that the intervals $G_{i}$ are disjoint we get that if $G_{q r} \not \subset E_{1}$, then $G_{q r} \cap E_{1}=\emptyset$. In this case we apply Corollary 4.8 once and the argument used define $q$ and (6.3) to prove the claim. If $G_{q r} \subset E_{1}$ let $q^{\prime} \geq 0$ be minimal so that for all $i$ with $q^{\prime} \leq i \leq q, G_{i r} \subset E_{1}$. Note that $I^{1}$ is well-inside $I^{0}$ (and therefore $I^{2}$ is well-inside $I^{1}$ ) and since $\hat{m}>1$ all critical points of $f^{r} \mid I^{1}$ are contained in $I^{2}$. Observe that since $I^{1}$ is well-inside $I^{0}$ and by (1) and (2) of Claim 2 that $\left(f^{r} \mid E_{1}\right)^{-1}\left(E_{1}\right)$ is well-inside $E_{1}$. We can apply Proposition 6.1 to $f^{r} \mid E^{1}$ and therefore we obtain $\lambda_{7}>0$ so that $f^{q^{\prime} r}(z) \in D_{\lambda_{7} \theta}\left(E_{1} \cap I^{1}\right)$. If $q^{\prime}=0,1$, then applying Corollary 4.8 gives (6.1). By the choice of $q^{\prime}, \quad G_{i r} \subset I^{\tilde{m}}$ for all $0 \leq i \leq q^{\prime}-2$. If $f^{\left(q^{\prime}-2\right) r}(z) \in D_{\theta}\left(I^{1}\right)$, then we can repeat the above argument choosing an integer $q<q^{\prime}-2$ as above. Hence the claim follows. $\checkmark$

Since $f^{r}$ is a composition of folding maps, $f^{r}\left(I^{\tilde{m}} \cap E_{1}\right)=f^{r}\left(I^{\tilde{m}} \cap E_{2}\right)=f^{r}\left(I^{\tilde{m}}\right)$. Let $J_{1}, J_{2}$ be the outermost connected components of $\left(f \mid I^{\tilde{m}}\right)^{-r}\left(I^{\tilde{m}}\right)$ that intersect $E_{1}, E_{2}$ respectively. Note that $c_{l}$ and $c_{r}$ are the turning points in the boundaries of $E_{1}$ and

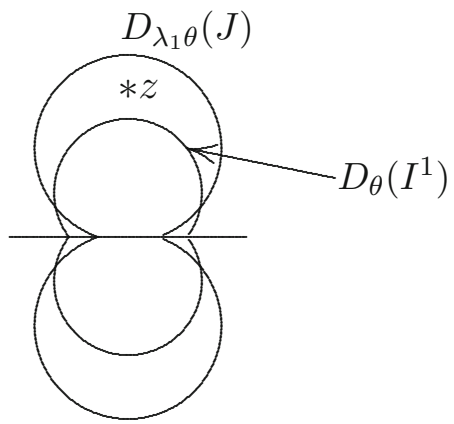

Fig. 11. The sets $D_{\theta}\left(I^{1}\right)$ and $D_{\lambda_{1} \theta}(J)$. The point $z$ is 'jumping' and we will capture it by $J$ 



Fig. 12. Two examples of cases where $\tilde{m}<\infty$ and one with $\tilde{m}=\infty$ and $I^{\tilde{m}}=\cap_{m \geq 0} I^{m}$. The intervals $E_{1}, E_{2}$ are marked in solid lines; the dashed lines refer to the intervals $J_{1}, J_{2}$ and the dotted lines are $J_{i j}$

$E_{2}$ respectively. Let $J_{1,1}$ and $J_{1,2}$ (if it exists) be the two outermost components of $\left(f \mid J_{1}\right)^{-r}\left(J_{1}\right)$ such that $f^{r} \mid J_{1, j}, j=1,2$ is monotone. See Fig. 12.

Claim 4. If $I^{\tilde{m}}$ is well-inside $I^{\tilde{m}-1}$ then (6.1) or (6.2) holds for $p^{\prime}=p-1$ and $I^{\prime}=I^{\tilde{m}}$.

Let us remark that when we say well-inside, we mean that $(1+2 \eta) I^{\tilde{m}} \subset I^{\tilde{m}-1}$ for some universal $\eta>0$, one is welcome to think of $\eta$ as being the constant $\delta>0$ from Lemma 3.8.

Proof of Claim 4. By Claim $2 \mathrm{w}$ have that $\left|I^{\tilde{m}}\right|$ is comparable to $\left|I^{0}\right|$, and by Claim 3 we have that $f^{p r}(z) \in D_{\hat{\lambda} \theta}(J) \backslash D_{\theta}\left(I^{1}\right)$. Hence by Lemma 4.5 there exists a constant $\lambda_{8} \in(0,1)$ so that $f^{p r}(z) \in D_{\lambda_{8} \theta}\left(I^{\tilde{m}}\right)$. Since $I^{\tilde{m}}$ is well-inside $I^{\tilde{m}-1}$, by Corollary 4.8 we obtain constant $\lambda_{9} \in(0,1)$, so that $f^{(p-1) r}(z) \in D_{\lambda_{9} \theta}\left(K^{\prime}\right)$ for some interval $K^{\prime}$ which is well-inside $I^{\tilde{m}}$ by Lemma 3.3 .

Let us describe the current situation. The interval $I^{\tilde{m}}$ is not well-inside $I^{\tilde{m}-1}$, and $\left|I^{\tilde{m}}\right|$ is comparable to $\left|I^{0}\right|$. Moreover, there exists a universal constant $\kappa_{3} \in(0,1)$ such that the intervals $J_{i}, J_{1, i}, i=1,2$ all have length at least $\kappa_{3}\left|I^{\tilde{m}}\right|$, the intervals $J_{1}, J_{2}$ and $J_{1,2}$ are not necessarily well-inside $I^{\tilde{m}}$, and $J_{1,2}$ is not necessarily well-inside $J_{1}$. Moreover, we can assume that the properties from Claim 3 hold (and in particular that $\left.G_{p r} \subset I^{\tilde{m}}\right)$.

Claim 5. There exists a constant $\kappa_{4} \in(0,1)$ so that each critical point $c^{\prime}$ of $f^{r} \mid I^{\tilde{m}}$ is contained in $\left(1+2 \kappa_{4}\right)^{-1} I^{\tilde{m}}$ and $f^{r}\left(J_{1,1}\right) \backslash J_{1,1}$ contains an interval of size $\kappa_{4}\left|J_{1,1}\right|$.

Proof of Claim 5. The first statement follows from the Claim 4, from Lemma 3.10 and since $f^{r} \mid I^{\tilde{m}}$ has no attracting fixed points. The second statement follows from the first one and Claim 2.

Claim 6. There exist $\delta_{0}>0, \lambda_{0} \in(0,1)$ depending on $\delta$ and for each $z \in U$ there exists an interval $K_{0}$ such that either (6.1) holds with $K=K_{0}$ and $\delta^{\prime}=\delta_{0}$ or there exists $p_{0}<p$ such that

$$
f^{p_{0} r}(z) \in D_{\lambda_{0} \theta}\left(K_{0}\right) \text { and } \quad G_{p_{0} r} \subset K_{0} \subset(1+2 \delta) K_{0} \subset I^{\tilde{m}} .
$$

Proof of Claim 6. If $G_{(p-1) r} \cap J_{1,1}=\emptyset$, then by Claim 5, $G_{(p-2) r}$ is well-inside $I^{\tilde{m}}$ and by applying Lemma 4.6 twice we obtain Eq. (6.4) for $p_{0}=p-2$ for some interval $K_{0} \supset G_{(p-2) r}$ which is well-inside $I^{\tilde{m}}$. So let $p_{1}<p$ be minimal so that $G_{i r} \cap J_{1,1} \neq \varnothing$ 
for $i=p_{1}, \ldots, p-1$. Suppose first that $p-p_{1} \geq 2$. We pull back twice using Corollary 4.8 , to obtain an interval $K_{1}$ which is well-inside $J_{1,1}$. One component of $f^{r}\left(J_{1,1}\right) \backslash J_{1,1}$ is a fundamental domain to one side of $K_{1}$ and one component of $I^{0} \backslash I^{1}$ is a fundamental domain to the other side. Claims 4 and 5 imply that the fundamental domains and the gap between them all have length comparable to $I^{\tilde{m}}$. So we can apply Proposition 6.1 to $f^{r} \mid J_{1,1} \rightarrow f^{r}\left(J_{1,1}\right) \subset I^{\tilde{m}}$ and thus obtain an interval $K^{\prime}$ which is well-inside $J_{1,1}$ and a constant $\lambda_{10}>0$ so that $f^{p_{1} r}(z) \in D_{\lambda_{10} \theta}\left(K^{\prime}\right)$. If $p_{1}=0$, then (6.1) holds and if $p_{1}>0$ (including the case $p-p_{1}<2$ ), then $G_{\left(p_{1}-1\right) r} \cap J_{1,1}=\emptyset$, and we argue as in beginning of the proof of this claim. $\checkmark$

Claim 7. If $\tilde{m}<\infty$, then there exist $0 \leq p^{\prime} \leq p_{0}, \delta^{\prime}>0$ and $\lambda^{\prime} \in(0,1)$ so that

$$
f^{p^{\prime} r}(z) \in D_{\lambda^{\prime} \theta}\left(K^{\prime}\right) \quad \text { and } \quad G_{p^{\prime} r} \subset K^{\prime} \subset\left(1+2 \delta^{\prime}\right) K^{\prime} \subset I^{\tilde{m}},
$$

where either $p^{\prime}=0$ or where $p^{\prime}>0$ and $\left(1+2 \delta^{\prime}\right) K^{\prime} \subset I^{\prime}$, where $I^{\prime}$ is a $\hat{\rho}$-nice interval in $I^{\tilde{m}}$ which is equal to $\mathcal{L}_{G_{p^{\prime} r}}\left(I^{\tilde{m}}\right)$ or to $\mathcal{L}_{G_{p^{\prime} r}}\left(\mathcal{L}_{G_{\left(p^{\prime}-1\right) r}}\left(I^{\tilde{m}}\right)\right)$.

Proof of Claim 7. Let $A_{k}$ be the component of $f^{-r}\left(I^{\tilde{m}}\right)$ containing $G_{k r}$. As before we can assume that $p_{0} \geq 1$. Let $p_{1} \leq p_{0}$ be minimal so that for all $i$ with $p_{1} \leq i<p_{0}, G_{i r}$ is a pullback of $G_{(i+1) r}$ through $J_{1,1}$. If $p_{1}$ is less than 3, then (6.1) holds by Corollary 4.8, so we will assume that $p_{1}>3$. Because of Claim 5 , as in Claim 6, we can apply Proposition 6.1 to $f^{r}: J_{1,1} \rightarrow f^{r}\left(J_{1,1}\right) \subset I^{\tilde{m}}$. If $p_{1}<p_{0}$, then this implies that there exist $\delta_{1}^{\prime}>0, \lambda_{1}^{\prime} \in(0,1)$ and an interval $K_{1}^{\prime}$ so that

$$
f^{p_{1} r}(z) \subset D_{\lambda_{1}^{\prime} \theta}\left(K_{1}^{\prime}\right)
$$

where $G_{p_{1} r} \subset K_{1}^{\prime} \subset\left(1+2 \delta_{1}^{\prime}\right) K_{1}^{\prime} \subset J_{1,1}=A_{p_{1}}$. So in any case we get that there exist $\delta_{2}^{\prime}>0, \lambda_{2}^{\prime} \in(0,1)$ and an interval $K_{2}^{\prime}$ so that

$$
f^{\left(p_{1}-1\right) r}(z) \subset D_{\lambda_{2}^{\prime} \theta}\left(K_{2}^{\prime}\right)
$$

where $G_{p_{1} r} \subset K_{2}^{\prime} \subset\left(1+2 \delta_{2}^{\prime}\right) K_{2}^{\prime} \subset A_{p_{1}-1}$ (here we use that $\left.G_{p_{1} r} \subset K_{1}^{\prime} \subset\left(1+2 \delta_{1}^{\prime}\right) K_{1}^{\prime}\right)$. To finish the proof, we show that we can pull back until we arrive in a return domain to a nice interval that is $\rho$-well-inside that nice interval,for a universal $\rho$, so that the return domain is $\hat{\rho}$-nice, for $\hat{\rho}>0$, universal, by Corollary 3.4.

In the remainder of the proof, when we say that an interval $J$ well-inside an interval $I$, we mean that $(1+2 \eta) J \subset I$, where $\eta$ depends only on the universal constants from Claims 2,3 and 5. Note that $A_{p_{1}-1}$ is well-inside $I^{\tilde{m}}$ unless $A_{p_{1}-1}=J_{1}$ or $J_{2}$. So unless $A_{p_{1}-1}=J_{1}$ or $J_{2}$ the claim follows taking $I^{\prime}=A_{p_{1}-1}$. If $A_{p_{1}-1}=J_{1}$, then we are done if $G_{\left(p_{1}-1\right) r}$ is contained in a landing domain to $J_{1}$ that is well-inside $J_{1}$. The only way that this does not happen is if $G_{\left(p_{1}-1\right) r} \subset J_{1,2}$. So suppose that this is the case. Under one more pullback $G_{\left(p_{1}-2\right) r}$ is either inside a return domain to $I^{\tilde{m}}$ that is well-inside $I^{\tilde{m}}$ or inside a return domain to $J_{1}$ that is well-inside $J_{1}$ or it is contained in $J_{2}$. We are done except in the last case, but then we do the following. If $A_{p_{1}-1}=J_{2}$, we pull back once more. If $A_{p_{1}-2}$ is not $J_{1}$ or $J_{2}$ we are done. If $A_{p_{1}-2}=J_{2}$ and $J_{2}$ is not monotone, then we are well-inside a landing domain to $J_{2}$ that is well-inside $J_{2}$. Suppose $J_{2}$ is monotone. Pulling back once more, we are done unless $A_{p_{1}-3}=J_{1}$. In this case we are done after one more pullback. So the claim and the proposition follow from Corollary 4.8. 


\section{Pulling Back a Poincaré Disc Along a Chain with Bounded Combinatorial Depth}

The results in this section apply to maps $f \in \mathcal{A}_{\underline{b}}$ and to asymptotically holomorphic extensions of maps $f \in \mathcal{A}_{b}^{3}$, as in Sect. 5.2. Once again, we recall that we always assume that the Standing Assumptions on p. 26 hold.

Let $I$ be a nice interval containing the critical point $c_{0}$, and let $m \geq 1$ be minimal so that $R_{I}\left(c_{0}\right) \notin I^{m}$. Notice that $m \geq \hat{m}$ (defined on p. 20). Recall,

$$
\mathcal{C}(I):= \begin{cases}I^{m} & \text { if } I \text { is non-terminating and } \\ I^{\infty} & \text { otherwise. }\end{cases}
$$

If $J$ is a return domain to an arbitrary nice interval $I$, and $\left\{G_{i}\right\}_{i=0}^{r}$ is the chain with $G_{r}=I$ and $G_{0}=J$ where $r$ is the return time of $J$ to $I$, we define

$$
\operatorname{Crit}(I ; J)=\left(\bigcup_{i=0}^{r-1} G_{i}\right) \cap \operatorname{Crit}(f) .
$$

Similarly, if $\mathbb{G}=\left\{G_{j}\right\}_{j=0}^{s}$ is an arbitrary chain such that the pullbacks of $G_{s}$ and $I$ are either nested or disjoint, $G_{0} \subset I$ and $0=n_{0}<n_{1}<\cdots<n_{p}=s$ are the integers with $G_{n_{i}} \subset I$, we define

$$
\operatorname{Crit}(I ; \mathbb{G})=\bigcup_{i=0}^{p-1} \operatorname{Crit}\left(I ; \mathcal{L}_{G_{n_{i}}}(I)\right) .
$$

For any nice interval $I$ and any critical point $c$ we define $k_{c}(I, \mathbb{G})=\inf \left\{k_{c} \geq 0\right.$ : there exists no $j=1,2, \ldots, s-1$ with $\left.G_{j} \subset \mathcal{C}^{k_{c}}\left(\hat{\mathcal{L}}_{c}(I)\right)\right\}$, where we take $\mathcal{C}^{0}\left(\hat{\mathcal{L}}_{c}(I)\right)=\hat{\mathcal{L}}_{c}(I)$ and

$$
k(I, \mathbb{G})=\sum_{c \in \operatorname{Crit}(I ; \mathbb{G})} k_{c}(I, \mathbb{G}) .
$$

The combinatorial depth of the chain $\mathbb{G}$ with respect to $I$ is defined to be $k(I ; \mathbb{G})$. Note that $k(I, \mathbb{G})$ is well-defined even if $I$ does not contain a critical point. If $J$ and $I$ are nice intervals with nested or disjoint pullbacks and $c \in J \subset I$, then we define

$$
k(I, J)=\min \left\{k \geq 0: \mathcal{C}^{k}(I) \subset J\right\}
$$

and take

$$
\hat{k}(I, J)=\sum_{c \in \operatorname{Crit}(f)} k\left(\hat{\mathcal{L}}_{c^{\prime}}(I), \hat{\mathcal{L}}_{c^{\prime}}(J)\right) .
$$

Remark. In the presence of terminating intervals it is possible for the combinatorial depth to be infinite. Let $\left\{G_{j}\right\}_{j=0}^{s}$ be a chain and suppose that $\mathcal{C}_{c}^{n}(I)$ is terminating. Then for all $k>n, \mathcal{C}_{c}^{k}(I)=\mathcal{C}_{c}^{n+1}(I)$, so if some interval $G_{k_{0}}$ is contained in $\mathcal{C}_{c}^{n+1}(I)$ we get that $k\left(I,\left\{G_{j}\right\}_{j=0}^{s}\right)$ is infinite. 
Proposition 7.1 (cf. [KSvS], Proposition 11.2). For each $\delta>0, k \geq 0$ and $N \geq 0$ there exist $\mu(k, N, \delta) \in(0,1)$ and $\delta^{\prime}>0$, and for each $\theta \in(0, \pi)$ there exists $\varepsilon>0$ so that the following holds. Let I be a $\delta$-nice interval with $|I|<\varepsilon$. Suppose that $\mathbb{G}:=\left\{G_{i}\right\}_{i=0}^{s}$ is a chain such that $G_{0}, G_{s}$ are nice intervals contained in $I$, the pullbacks of $G_{s}$ and I are nested or disjoint, the intervals $G_{0}, \ldots, G_{s-1}$ are pairwise disjoint and $G_{0} \cap \omega\left(c_{0}\right) \neq \varnothing$. Assume that

$$
k(I, \mathbb{G}) \leq k \text { and } \# \operatorname{Crit}(I ; \mathbb{G}) \leq N .
$$

Let $\hat{G}_{s}$ be an interval with $G_{s} \subset \hat{G}_{s} \subset(1+2 \delta) \hat{G}_{s} \subset I$. Let $V=D_{\theta}\left(\hat{G}_{s}\right) \cap \mathbb{C}_{G_{s}}$ and $U_{i}=\operatorname{Comp}_{G_{i}} f^{-(s-i)}(V)$ for $i=0, \ldots, s$. Then, there exists an interval $\hat{I} \supset G_{0}$ with $\left(1+2 \delta^{\prime}\right) \hat{I} \subset I$ such that

$$
U_{0} \subset D_{\mu(k, N, \delta) \theta}(\hat{I}) .
$$

Proof. The proof of this proposition goes by induction on $(N, k)$ with the lexicographical ordering. If $N=0$, then all the branches are diffeomorphisms, so the proposition follows because the sum of the lengths of the intervals $\hat{G}_{0}, \ldots, \hat{G}_{s-1}$ is uniformly bounded. This can be seen as follows: let $F: \mathcal{J} \rightarrow I$ be equal to the first return map to $I$ restricted to its diffeomorphic branches. Let $J_{n}$ be a domain of $F^{n}$ and $J_{n+1} \subset J_{n}$ a domain of $F^{n+1}$. Since $F^{n}: J_{n} \rightarrow I$ is a diffeomorphism, by Theorem 3.2 (2) $F^{n} \mid J_{n}$ has bounded distortion. Since each component of $\mathcal{J}$ is $\delta$-well-inside $I$, it follows that there exists $\kappa \in(0,1)$ depending only on $\delta$ such that $\left|J_{n+1}\right| \leq \kappa\left|J_{n}\right|$. It follows that the sum of the lengths of the intervals $\hat{G}_{i}$ contained in $I$ is universally bounded, and now Theorem 3.2 (2) implies that the sum of the lengths of the intervals $\hat{G}_{i}$ is universally bounded.

Assume now that the statement holds for all $\left(N^{\prime}, k^{\prime}\right)$ for which either $N^{\prime}<N$ or $N^{\prime}=N$ and $k^{\prime}<k$. We will prove that the statement holds for $(N, k)$. Let $\mathbb{G}=\left\{G_{j}\right\}_{j=0}^{s}$ be a chain as above with $k(I, \mathbb{G})=k$ and $\# \operatorname{Crit}(I ; \mathbb{G})=N$.

Without loss of generality we can assume that $I \cap \operatorname{Crit}(f) \neq \emptyset$. If this is not the case we can define $t<s$ to be maximal so that $G_{t} \subset \mathcal{L}_{c}(I)$, for a critical point $c \in \operatorname{Crit}(f)$. Suppose that for no $t^{\prime}, t<t^{\prime}<s$, we have that $G_{t^{\prime}} \subset I$, then $G_{s}$ is the first entry of $G_{t}$ into $I$, so we can pull back to time $t$ using Lemma 4.8. Otherwise, let $t<t^{\prime}<s$ be minimal so that $G_{t^{\prime}} \subset I$. Using the argument for $N=0$, we pull back from time $s$ to time $t^{\prime}$ and using Lemma 4.8 to pull back from time $t^{\prime}$ to time $t$. In either case, we obtain

$$
U_{t} \subset D_{\lambda_{0} \theta}\left(H_{t}\right) \cap \mathbb{C}_{G_{t}},
$$

where $G_{t} \subset \hat{G}_{t} \subset H_{t} \subset\left(1+2 \delta_{0}\right) H_{t} \subset \mathcal{L}_{c}(I)$, where $\lambda_{0} \in(0,1)$ and $\delta_{0}>0$ depend on $\delta$. Since $I$ is $\delta$-nice, Lemma 3.3 implies that after shrinking $\delta_{0}$, if necessary, $\mathcal{L}_{c}(I)$ is $\delta_{0}$-nice.

From now on we will assume that $I$ contains a critical point $c$. Let $I^{1}=\mathcal{L}_{c}(I)$. Let $s^{\prime}<s$ be maximal such that $G_{s^{\prime}} \subset I$. Applying Lemma 4.8 we get that

$$
U_{s^{\prime}} \subset D_{\lambda_{1} \theta}\left(H_{s^{\prime}}\right) \cap \mathbb{C}_{G_{s^{\prime}}},
$$

where $G_{s^{\prime}} \subset \hat{G}_{s^{\prime}} \subset H_{s^{\prime}} \subset\left(1+2 \delta_{1}\right) H_{s^{\prime}} \subset \mathcal{L}_{G_{s^{\prime}}}(I)$, where $\lambda_{1} \in(0,1)$ and $\delta_{1}>0$ depend on $\delta$. Depending on the position of $G_{S^{\prime}}$ we have two cases.

Case 1. $G_{s^{\prime}} \subset I \backslash I^{1}$. Let $J=\mathcal{L}_{G_{s^{\prime}}}(I)$. By Corollary 3.4 , there exists $\delta^{\prime \prime}>0$ so that $J$ is $\delta^{\prime \prime}$-nice. Define $s_{1}<s^{\prime}$ minimal such that $G_{s_{1}} \subset J$. If there exists no such $s_{1}$, then $G_{s^{\prime}}$ is the first entry of $G_{0}$ to $J$ and the proposition follows from Lemma 4.8. 
Let us assume $s_{1}$ is defined. The structure of the real puzzle and the fact that $c$ escapes $I^{1}$ imply that $\mathcal{L}_{c}(J) \subset \mathcal{C}_{c}(I)$. It follows easily from this fact and the definition of $k$ that if any of the intervals $G_{s_{1}}, \ldots, G_{s^{\prime}}$ enters $\mathcal{C}_{c}(I)$, then

$$
k\left(J,\left\{G_{j}\right\}_{j=s_{1}}^{s^{\prime}}\right) \leq k\left(I,\left\{G_{j}\right\}_{j=0}^{s}\right)-1=k-1,
$$

and if none of the intervals $G_{s_{1}}, \ldots, G_{s^{\prime}}$ enters $\mathcal{C}_{c}(I)$, then $\operatorname{Crit}\left(J,\left\{G_{j}\right\}_{j=s_{1}}^{s^{\prime}}\right)<N$. In either case, replacing $I$ by $J$ we may apply the induction hypothesis to the chain $\left\{G_{j}\right\}_{j=s_{1}}^{s^{\prime}}$ to obtain

$$
U_{s_{1}} \subset D_{\lambda_{2} \theta}\left(H_{s_{1}}\right) \cap \mathbb{C}_{G_{s_{1}}}
$$

where $G_{s_{1}} \subset \hat{G}_{s_{1}} \subset H_{s_{1}} \subset\left(1+2 \delta_{2}\right) H_{s_{1}} \subset J$, where $\lambda_{2} \in(0,1)$ and $\delta_{2}>0$ depend on $\delta, N$ and $k$. By the minimality of $s_{1}$ and Lemma 4.8 we have that

$$
U_{0} \subset D_{\lambda_{3} \theta}\left(H_{0}\right) \cap \mathbb{C}_{G_{0}},
$$

where $G_{0} \subset \hat{G}_{0} \subset H_{0} \subset\left(1+2 \delta_{3}\right) H_{0} \subset I$, with $\delta_{3}>0$ and $\lambda_{3} \in(0,1)$ depending only on $\delta_{2}$. This completes the proof in this case.

Case 2. $G_{S^{\prime}} \subset I^{1}$. There are two possibilities: either the return map to $I$ restricted to $I^{1}$ is monotone or it has a turning point.

If the return map is monotone, let $0 \leq t^{\prime \prime} \leq s^{\prime}$ be minimal so that $G_{t^{\prime \prime}} \subset I^{1}$ and $G_{j} \cap\left(I \backslash I^{1}\right)=\emptyset$ for all $j=t^{\prime \prime}, \ldots, s^{\prime}$. We apply Proposition 6.1 to find $\lambda^{\prime \prime} \in(0,1)$ so that

$$
U_{t^{\prime \prime}} \subset D_{\lambda^{\prime \prime} \theta}\left(I^{2}\right)
$$

If $t^{\prime \prime}=0$, we have proved the proposition. If $t^{\prime \prime}>0$, there exists $0 \leq q<t^{\prime \prime}$ maximal such that $G_{q} \subset I$. By definition of $q$ we have that $G_{q} \subset I \backslash I^{1}$ and repeating Case 1, we obtain the induction statement.

Assume that the return map to $I$ restricted to $I^{1}$ is not monotone. Let $0 \leq s^{\prime \prime}<s^{\prime}$ be maximal such that $G_{s^{\prime \prime}} \subset I$. If $s^{\prime \prime}=0$ the proposition follows from Lemma 4.8, so suppose $0<s^{\prime \prime}$. If $G_{s^{\prime \prime}} \subset I \backslash I^{1}$, then the proposition follows from Case 1, so let us assume $G_{s^{\prime \prime}} \subset I^{1}$. Let $r_{1} \leq s^{\prime}$ be maximal such that $G_{r_{1}} \subset \hat{\mathcal{L}}_{c^{\prime}}(I)$, where $c^{\prime} \in \operatorname{Crit}(f)$ is a turning point. Note that $s^{\prime \prime}<r_{1} \leq s^{\prime}$. Let $J=\mathcal{L}_{c^{\prime}}(I)$. By Lemma 4.8, we have that

$$
U_{r_{1}} \subset D_{\lambda_{1}^{\prime} \theta}\left(H_{r_{1}}\right) \cap \mathbb{C}_{G_{r_{1}}}
$$

where $G_{r_{1}} \subset \hat{G}_{r_{1}} \subset H_{r_{1}} \subset\left(1+2 \delta_{1}^{\prime}\right) H_{r_{1}} \subset J$, and $\lambda_{1}^{\prime} \in(0,1)$ and $\delta_{1}^{\prime}>0$ both depend only on $\delta$. Once again, making $\delta_{1}^{\prime}$ smaller, if necessary, we may assume that $J$ is a $\delta_{1}^{\prime}$-nice. Let $q^{\prime} \leq r_{1}$ be minimal such that $G_{q} \subset J$ and let $r_{1}^{\prime}<r_{1}$ be maximal such that $G_{r_{1}^{\prime}} \subset J$. If $r_{1}^{\prime}=q^{\prime}$, the proposition follows from Lemma 4.8, so we assume $q^{\prime}<r_{1}^{\prime}$. Then we have two possibilities: either $G_{r_{1}^{\prime}} \subset J \backslash J^{1}$ or $G_{r_{1}^{\prime}} \subset J^{1}$. If the first holds, then we are in Case 1 and we obtain the induction statement.

Assume $G_{r_{1}^{\prime}} \subset J^{1}$. Let $t_{1} \leq r_{1}^{\prime}$ be minimal so that $G_{t_{1}} \subset J^{1}$ and $G_{j} \cap\left(J \backslash J^{1}\right)=\emptyset$ for all $t_{1} \leq j \leq r_{1}^{\prime}$ with $G_{j} \subset J$. Note that if $J \ni c$ is terminating, since $G_{t_{1}} \cap \omega\left(c_{0}\right) \neq \emptyset$, then $G_{t_{1}} \subset J^{\infty}$, so $k\left(\mathcal{L}_{c}(I), \mathbb{G}\right)$ is infinite. Hence we have that $J$ is non-terminating. By Proposition 6.3 we obtain $\alpha, \hat{\rho}>0, \lambda_{2}^{\prime} \in(0,1)$ and for each $z \in U_{t_{1}}$ an interval $K$ so that either 
(a) $f^{t_{1}}(z) \in D_{\lambda_{2}^{\prime} \theta}(K)$ and $G_{t_{1}} \subset K \subset(1+2 \alpha) K \subset J$ or

(b) there exist $r_{2}$ with $t_{1} \leq r_{2} \leq r_{1}^{\prime}$ and a $\hat{\rho}$-nice interval $J^{\prime} \subset J^{\hat{m}}$, so that $f^{r_{2}}(z) \in$ $D_{\lambda_{2}^{\prime} \theta}(K)$ and $G_{r_{2}} \subset K \subset(1+2 \alpha) K \subset J^{\prime}$.

If (a) holds, then the same argument used for $t^{\prime \prime}$ in the monotone case proves the proposition. If (b) holds, then by the definition of $\tilde{m}$, there exists $c^{\prime \prime} \in \operatorname{Crit}(J, \mathbb{G})$ which enters $J \backslash J^{1}$ before it enters $J^{\tilde{m}} \supset J^{\prime}$. Let $q^{\prime}$ be minimal such that for every $q^{\prime} \leq j \leq r_{2}$ with $G_{j} \subset J$ we have that $G_{j} \subset J^{\tilde{m}}$. If we take $q^{\prime} \leq r \leq r_{2}$ minimal so that $G_{r} \subset J^{\prime}$, then $c^{\prime \prime} \notin \operatorname{Crit}\left(J^{\prime},\left\{G_{j}\right\}_{j=r}^{r_{2}}\right)$, and therefore \#Crit $\left(J^{\prime},\left\{G_{j}\right\}_{j=r}^{r_{2}}\right)<N$. Replacing $I$ with $J^{\prime}$, and applying the induction hypothesis to the chain $\left\{G_{j}\right\}_{j=r}^{r_{2}}$, we have that $f^{r}(z) \in D_{\lambda_{3}^{\prime} \theta}\left(\hat{J}^{\prime}\right)$, where $\hat{J}^{\prime}$ is an interval such that $G_{r} \subset \hat{J}^{\prime} \subset\left(1+2 \alpha^{\prime}\right) \hat{J}^{\prime} \subset J^{\prime}$ and $\lambda_{3}^{\prime} \in(0,1)$ depend only on $\alpha, N$ and $k$. If $G_{0}, \ldots, G_{r-1}$ avoid $J^{\prime}$, then Lemma 4.8 completes the proof of the proposition. Otherwise, there exists $r_{3}<q^{\prime}$ so that $G_{r_{3}} \subset J \backslash J^{1}$. Let $r_{3}$ be maximal with respect to this property. Then $G_{r_{3}}, \ldots, G_{r-1}$ avoid $J^{\prime}$ and so applying Lemma 4.8 we obtain $f^{r_{3}}(z) \in D_{\lambda^{\prime \prime} \theta}\left(\hat{J}^{\prime \prime}\right)$ for some interval $\hat{J}^{\prime \prime}$ satisfying $G_{r_{3}} \subset \hat{J}^{\prime \prime} \subset\left(1+2 \alpha^{\prime \prime}\right) \hat{J}^{\prime \prime} \subset J$ where $\alpha^{\prime \prime}>0$ depends on $\alpha^{\prime}$, and $\lambda^{\prime \prime} \in(0,1)$ depends on $\alpha^{\prime}, N$ and $k$. Applying Case 1 , replacing $s$ with $r_{3}$ completes the proof in this case.

\section{Pulling Back a Poincaré Disc One Step Along the Enhanced Nest}

The results in this section apply to maps $f \in \mathcal{A}_{\underline{b}}$ and to asymptotic holomorphic extensions of maps $f \in \mathcal{A}_{b}^{3}$, as in Sect. 5.2. We recall that we always assume that the Standing Assumptions on p. $2 \overline{6}$ hold.

First, we state and prove a lemma which relates a bound on the scaling factors between two levelswith some combinatorial information.

Lemma 8.1. For each $v \geq 1$, there exists $K \in \mathbb{N}$ with the following properties. Assume that $I_{i}$ and $I_{i+1}$ are non-terminating and that both

$$
\left|I_{i}\right| /\left|I_{i+1}\right| \text { and }\left|I_{i+1}\right| /\left|I_{i+2}\right| \leq v .
$$

Then

$$
k\left(I_{i}, I_{i+1}\right) \text { and } k\left(\mathcal{L}_{c}\left(I_{i}\right), \mathcal{L}_{c}\left(I_{i+1}\right)\right) \leq K \text { for each } c \in \operatorname{Crit}(f) \cap \omega\left(c_{0}\right) .
$$

Proof. If $k\left(I_{i}, I_{i+1}\right)$ is large, then by Lemma 3.8, $\left|I_{i}\right| /\left|I_{i+1}\right|$ is large. If $c$ is a critical point and $k\left(\mathcal{L}_{c}\left(I_{i}\right), \mathcal{L}_{c}\left(I_{i+1}\right)\right)$ is large, then by Lemma $3.15\left|\mathcal{L}_{c_{0}} \mathcal{L}_{c}\left(I_{i}\right)\right| /\left|\mathcal{L}_{c_{0}} \mathcal{L}_{c}\left(I_{i+1}\right)\right|$ is large too. By construction, we have that

$$
I_{i} \supset \mathcal{L}_{c_{0}} \mathcal{L}_{c}\left(I_{i}\right) \supset \Gamma\left(I_{i}\right) \supset I_{i+1}
$$

and

$$
I_{i+1} \supset \mathcal{L}_{c_{0}} \mathcal{L}_{c}\left(I_{i+1}\right) \supset \Gamma\left(I_{i+1}\right) \supset I_{i+2} .
$$

So, if $\left|\mathcal{L}_{c_{0}} \mathcal{L}_{c}\left(I_{i}\right)\right| /\left|\mathcal{L}_{c_{0}} \mathcal{L}_{c}\left(I_{i+1}\right)\right|$ is large, and since $I_{i} \supset \mathcal{L}_{c_{0}} \mathcal{L}_{c}\left(I_{i}\right) \supset I_{i+1} \supset$ $\mathcal{L}_{c_{0}} \mathcal{L}_{c}\left(I_{i+1}\right) \supset I_{i+2}$, either $\left|I_{i}\right| /\left|I_{i+1}\right|$ or $\left|I_{i+1}\right| /\left|I_{i+2}\right|$ is large, which contradicts our assumption. 
8.1. The renormalizable case. In this subsection, we deal with pullbacks along consecutive intervals of the enhanced nest in the presence of terminating intervals.

Figures 13 and 14 show how these pullbacks are structured.

In the next proposition we will deal with the first step of the pullback when $I_{i-1}$ is non-terminating and $I_{i}$ is terminating, see Fig. 13.

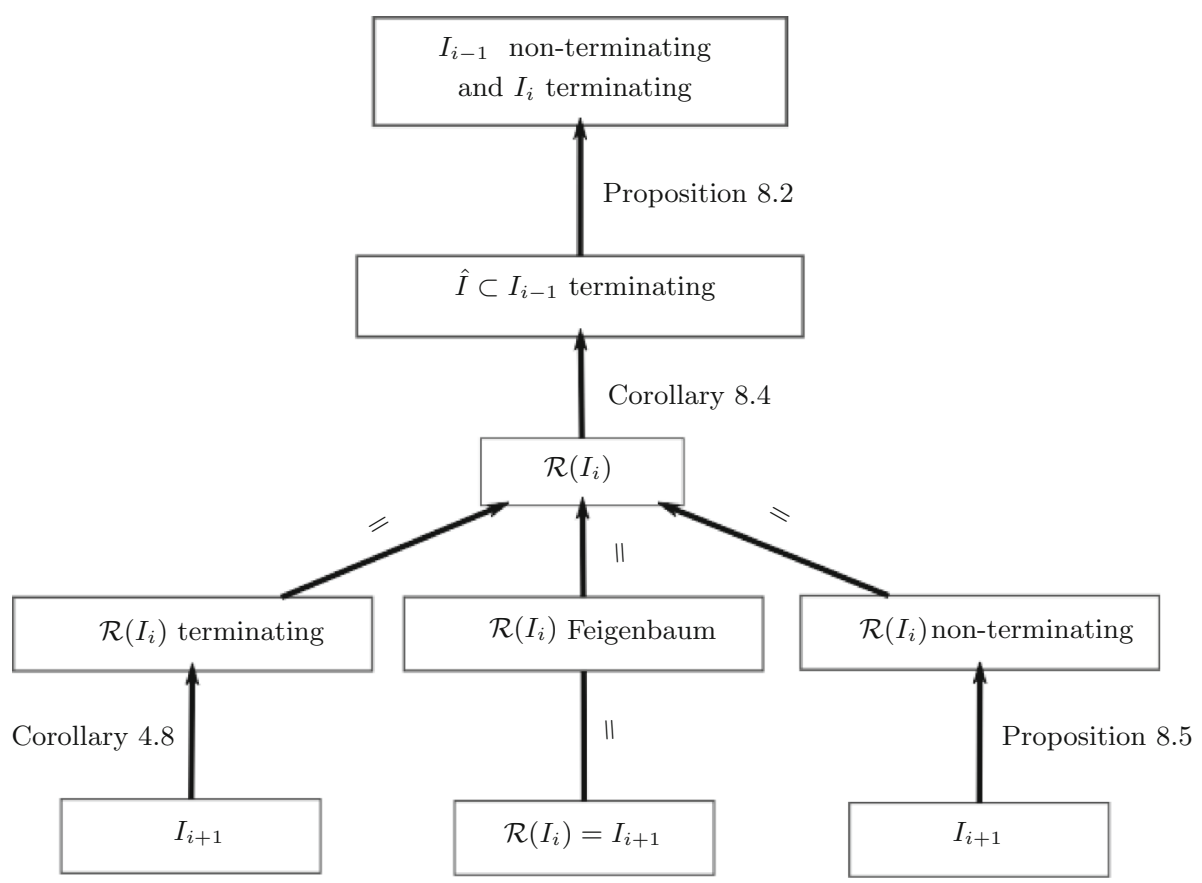

Fig. 13. Pulling back a Poincaré disk based on $I_{i-1}$ to one based on $I_{i+1}$ when $I_{i-1}$ is non-terminating and $I_{i}$ is terminating. We start at the top of the diagram and the arrows indicate pulling back. The $=$ symbol indicates that there is nothing to do

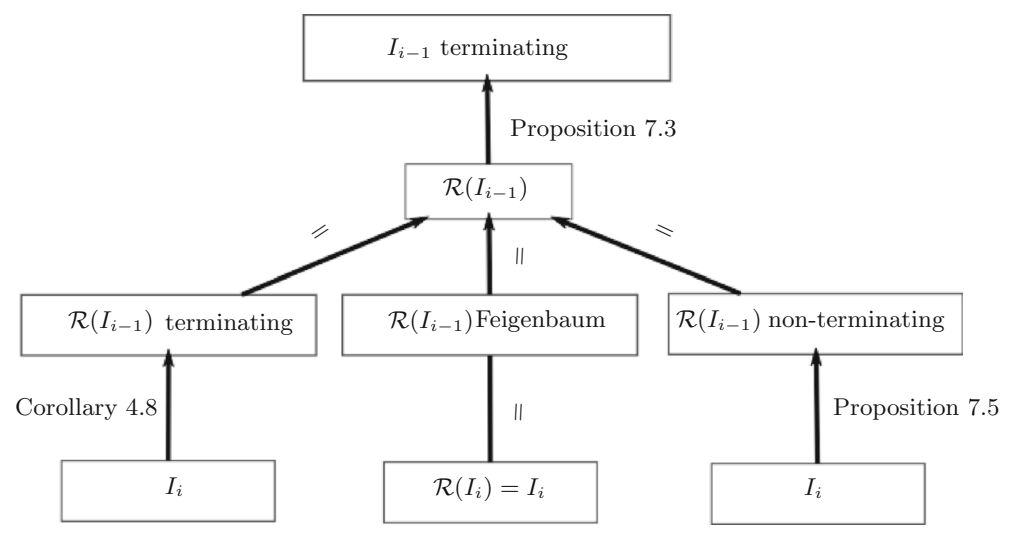

Fig. 14. Pulling back a Poincaré disk based on $I_{i-1}$ to one based on $I_{i}$ when $I_{i-1}$ is terminating. We start at the top of the diagram and the arrows indicate pulling back. The $=$ symbol indicates that there is nothing to do 
Proposition 8.2. For any $v \geq 1$ there exists $\lambda \in(0,1)$, and for each $\theta \in(0, \pi)$ there exists $\varepsilon>0$ such that the following holds. Suppose that $I_{i-1}$ is non-terminating, $I_{i}$ is terminating, $\left|I_{i-1}\right|<\varepsilon$ and

$$
\frac{\left|I_{i-2}\right|}{\left|I_{i+1}\right|}<v .
$$

Let $r$ be such that $\left.f^{r}\right|_{I_{i}^{\infty}}=R_{I_{i}^{\infty}}$. Let $\left\{G_{j}\right\}_{j=0}^{s}$ be a disjoint chain with $G_{0} \subset I_{i}^{\infty}$ and $G_{0} \cap \omega\left(c_{0}\right) \neq \varnothing$. Assume that $G_{s_{i-1}} \subset I_{i-1}$ for some $s_{i-1}>2 r$. Then there exists $s_{i}^{\prime}<s_{i-1}$ with $G_{s_{i}^{\prime}} \subset I_{i}^{\infty}$ such that if

$$
U_{s_{i-1}}=D_{\theta}\left(I_{i-1}\right) \cap \mathbb{C}_{G_{s_{i-1}}} \text { and } U_{0}=\operatorname{Comp}_{G_{0}} f^{-s_{i-1}}\left(U_{s_{i-1}}\right) \text {, }
$$

then there is an interval $K$ which is well-inside $\hat{I}$ such that

$$
U_{s_{i}^{\prime}} \subset D_{\lambda \theta}(K)
$$

where $\hat{I} \supset I_{i-1}^{\infty}$ is the largest terminating interval in the principal nest

$$
I_{i-1}=I_{i-1}^{0} \supset I_{i-1}^{1} \supset \cdots .
$$

Moreover, there are at most two integers $j$ with $s_{i}^{\prime} \leq j \leq s_{i-1}$ and $G_{j} \cap I_{i} \neq \emptyset$.

Remark. The time $s_{i}^{\prime}$ is chosen so that the base of the Poincare domain containing $U_{s_{i}^{\prime}}$ is contained in $\hat{I}$. Pulling back to time $s_{i}^{\prime}$ is the first step needed to eventually get a Poincaré disk based on $I_{i+1}$ (see Fig. 13).

Proof. Let $I_{i-1}=I^{0} \supset I^{1} \supset I^{2} \supset I^{3} \supset \cdots$ be the principal nest about $c_{0}$. Let $m(0)=0$ and let $m(0)<m(1)<m(2)<m(3) \cdots<m(l)$ be the levels the of principal nest such that $R_{I^{m(i)-1}}\left(c_{0}\right) \notin I^{m(i)}, i=1,2, \ldots, l$. Note that $l<\infty$ since $I_{i}$ is terminating. By definition $I^{m(l)}$ is terminating, so $I^{m(l)} \supset I_{i}^{\infty}$ and the return time of $I^{m(l)+1}$ to $I^{m(l)}$ is $r$. We will begin by defining a time $j_{0}$ from which we will be able to pull back to a time $s_{i}^{\prime}$, as in the statement of the proposition, along a bounded number of monotone branches in the principal nest about $c_{0}$ and first return maps. We will first pick a sequence of times $k_{i} \leq k_{i}^{\prime}$ that give us good control of the orbit of $c_{0}$ in the principal nest, $I^{0} \supset I^{1} \supset I^{2} \supset \cdots \supset I^{m(l)}$.

Let $k_{l}<s_{i-1}$ be maximal such that $G_{k_{l}} \subset I_{i}^{\infty}$. Let

$$
k_{l}<k_{l-1} \leq k_{l-1}^{\prime}<k_{l-2} \leq \cdots<k_{0} \leq k_{0}^{\prime}<k^{*} \leq j_{0} \leq s_{i-1}
$$

be defined as follows. See Fig. 15 for an illustration of this sequence. Let $k_{l-1}>k_{l}$ be minimal such that $f^{k_{l-1}}\left(c_{0}\right) \in I^{m(l)-1}$. Since $G_{k_{l}} \subset I_{i}^{\infty}, k_{l-1}$ coincides with the minimal time $>k_{l}$ such that $G_{k_{l-1}} \subset I^{m(l)-1}$ and $f^{k_{l-1}}\left(c_{0}\right) \notin I^{m(l)}$. Assuming that $k_{j}$ is defined, let $k_{j}^{\prime}$ be maximal such that for all $k, k_{j} \leq k \leq k_{j}^{\prime}$, if $f^{k}\left(c_{0}\right) \in I^{m(j)}$, then $f^{k}\left(c_{0}\right) \in \mathcal{L}_{c_{0}}\left(I^{m(j)}\right)$, and define $k_{j-1}=k_{j}^{\prime}+n_{j}$ where $n_{j}$ is the return time of

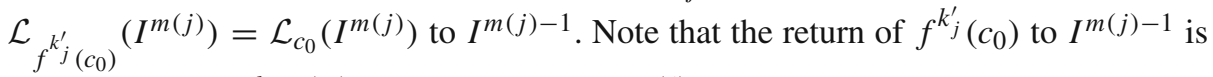
non-central, i.e. $f^{k_{j-1}\left(c_{0}\right)}$ is not contained in $I^{m(j)}$. With $k_{0}^{\prime}$ defined, we set $k_{*}=k_{0}^{\prime}+q_{0}$, where $q_{0}$ is the return time of $\mathcal{L}_{f^{k_{0}^{\prime}\left(c_{0}\right)}}\left(I^{m(0)}\right)=I^{m(0)+1}$ to $I^{m(0)}$. Notice that $f^{k_{*}}\left(c_{0}\right) \notin$ 


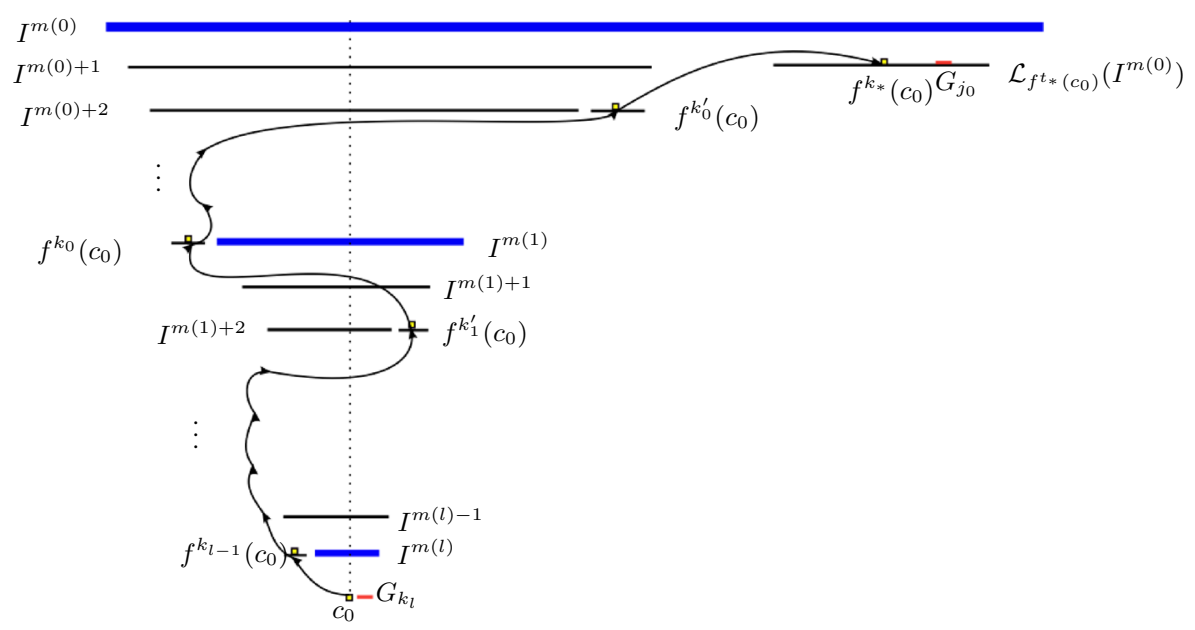

Fig. 15. Finding $k_{*}$

$I^{m(0)+1}$. Let $j_{0}, s_{i-1}-r<j_{0} \leq s_{i-1}$ be such that $G_{j_{0}} \subset \mathcal{L}_{f^{k *}\left(c_{0}\right)}\left(I_{i}^{\infty}\right)$; remember $r$ is the period of $I_{i}^{\infty}$. Since $s_{i-1}>2 r$, we know that $j_{0} \geq k_{*}$.

The pullback of $G_{j_{0}}$ will follow the same path as the pullback of $f^{k_{*}}\left(c_{0}\right)$ since they are both contained in the orbit of $I_{i-1}^{\infty}$. We will use this property below Claim 2, see Fig. 16.

Combinatorial Remark 1. Note that by the definition of $j_{0}$ there exists at most one interval $G_{j}$ with $j_{0} \leq j \leq s_{i-1}$ with $G_{j} \subset I_{i}^{\infty}$.

We start by pulling back along the first part of the chain, from time $s_{i-1}$ to time $j_{0}$. The loss of angle along this segment of the chain will be controlled by Proposition 7.1, so we need find a way to split the chain into smaller segments, each of them with bounded combinatorial depth. The argument is complicated by the fact that the chain may enter a terminating component of the landing map to $I_{i}$ that contains a critical point and therefore has infinite combinatorial depth. Let us study the combinatorial depth of the chain $\left\{G_{j}\right\}_{j=j_{0}}^{s_{i-1}}$ with respect to the interval $I_{i-1}$. By construction, there exists at most one interval $G_{j}$ with $j_{0}<j \leq s_{i-1}$ and $G_{j} \subset I_{i-1}^{\infty}$. Since $I_{i} \subset I_{i-1}^{\infty}$, for each $c \in \operatorname{Crit}\left(I_{i-1} ;\left\{G_{j}\right\}_{j=j_{0}}^{s_{i-1}}\right)$, there exists at most one interval $G_{j}$ with $j_{0}<j<s_{i-1}$ such that $G_{j} \subset \hat{\mathcal{L}}_{c}\left(I_{i}\right)$. Let $t_{1}$ be maximal so that $j_{0}<t_{1}<s_{i-1}$ and $\hat{\mathcal{L}}_{G_{t_{1}}}\left(I_{i}\right)$ contains a critical point. If $t_{1}$ is not defined,let $t_{1}=j_{0}$. Let $t_{1}^{\prime}>t_{1}$ be minimal such that $G_{t_{1}^{\prime}} \subset I_{i-1}$ and let $\mathbb{G}=\left\{G_{j}\right\}_{j=t_{1}^{\prime}}^{s_{i-1}}$. There exist two possibilities either

(i) $k\left(I_{i-1}, \mathbb{G}\right)$ is finite or

(ii) $k\left(I_{i-1}, \mathbb{G}\right)=\infty$.

First, we estimate the combinatorial depth of the chain $\mathbb{G}$ if (i) holds.

Claim 1. If $k\left(I_{i-1}, \mathbb{G}\right)$ is finite there exists $a=a(v)>0$ such that $k\left(I_{i-1}, \mathbb{G}\right)<a$.

Assume $k\left(I_{i-1}, \mathbb{G}\right)>a$, where $a$ will be chosen later. By the definition of combinatorial depth, there exists $c^{\prime} \in \operatorname{Crit}\left(I_{i-1} ; \mathbb{G}\right)$ such that $k\left(I_{i-1},\left\{G_{j}\right\}_{j=n_{c^{\prime}}}^{m_{c^{\prime}}}\right)>a / b$. 


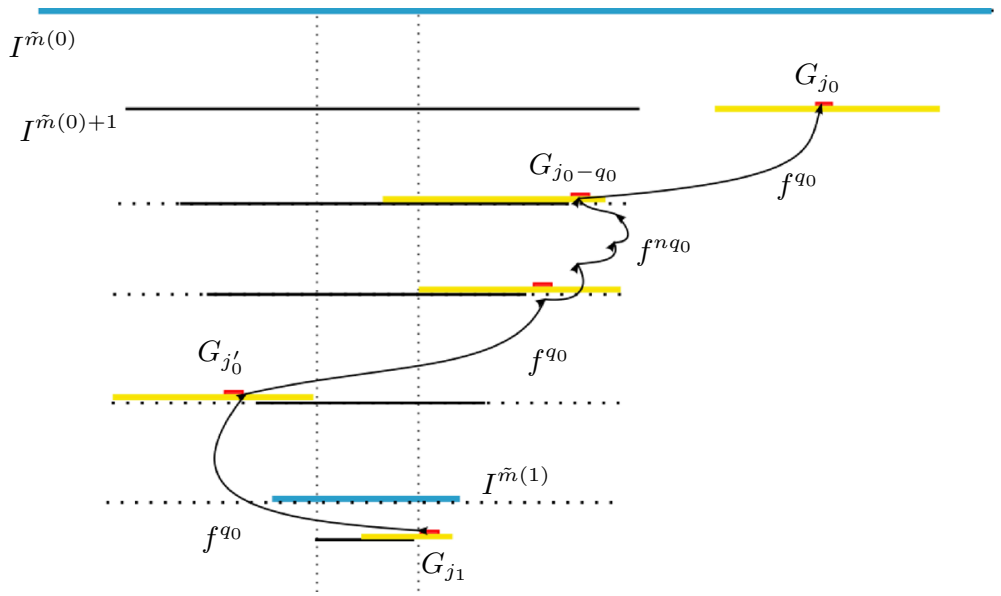

Fig. 16. Pulling back from time $j_{0}$ to $j_{1}$

Let $a_{*}$ be the integer part of $a / b$ and let $c^{\prime} \in \operatorname{Crit}\left(I_{i-1} ; \mathbb{G}\right)$ be so that $m_{c^{\prime}}$ is maximal and $k\left(I_{i-1},\left\{G_{j}\right\}_{j=n_{c^{\prime}}}^{m c_{c^{\prime}}}\right)>a_{*}$. By the definition of combinatorial depth, there exists $G_{j} \subset \mathcal{C}^{a_{*}}\left(\hat{\mathcal{L}}_{c^{\prime}}\left(I_{i-1}\right)\right)$. Assume $j^{\prime}$ is maximal with $t_{1}^{\prime} \leq j^{\prime} \leq s_{i-1}$ and $G_{j^{\prime}} \subset \mathcal{C}^{a_{*}}\left(\hat{\mathcal{L}}_{c^{\prime}}\left(I_{i-1}\right)\right)$; since $j^{\prime}>t_{1}, \mathcal{L}_{c^{\prime}}\left(I_{i}\right) \subset \mathcal{C}^{a_{*}}\left(\hat{\mathcal{L}}_{c^{\prime}}\left(I_{i-1}\right)\right)$. Since $k\left(I_{i-1}, \mathbb{G}\right)$ is finite none of the intervals $\mathcal{C}^{n}\left(\hat{\mathcal{L}}_{\mathcal{C}^{\prime}}\left(I_{i-1}\right)\right)$ is terminating for $1 \leq n \leq a_{*}$. By Lemma 3.8 there exists $\rho>0$ such that for any $1<n<a_{*}$

$$
(1+2 \rho) \mathcal{C}^{n+1}\left(\hat{\mathcal{L}}_{c^{\prime}}\left(I_{i-1}\right)\right) \subset \mathcal{C}^{n}\left(\hat{\mathcal{L}}_{c^{\prime}}\left(I_{i-1}\right)\right) .
$$

If $c^{\prime}=c_{0}$, and $a$ is sufficiently big, so $a_{*}$ is sufficiently big, this would imply $\left|I_{i-1}\right| /\left|I_{i+1}\right|>v$. So let us assume $c^{\prime} \neq c_{0}$. For each $n<a_{*}$ let $p_{n}$ be minimal such that $f^{p_{n}}\left(c_{0}\right) \subset \mathcal{C}^{n}\left(\hat{\mathcal{L}}_{c^{\prime}}\left(I_{i-1}\right)\right) ; p_{n}$ is the first entry time of $c_{0}$ to $\mathcal{C}^{n}\left(\hat{\mathcal{L}}_{c^{\prime}}\left(I_{i-1}\right)\right)$. Let $C_{n}^{n}$ and $C_{n+1}^{n}$ be the pullbacks of $\mathcal{C}^{n}\left(\hat{\mathcal{L}}_{c^{\prime}}\left(I_{i-1}\right)\right)$ and $\mathcal{C}^{n+1}\left(\hat{\mathcal{L}}_{c^{\prime}}\left(I_{i-1}\right)\right)$ containing $c_{0}$ along the orbit $c_{0}, f\left(c_{0}\right), \ldots, f^{p_{n}}\left(c_{0}\right)$, respectively. By Theorem $3.2(1)$ there exists $\rho^{\prime}>0$ such that for any $n<a_{*}$

$$
\left(1+2 \rho^{\prime}\right) C_{n+1}^{n} \subset C_{n}^{n}
$$

Note that $a_{n} \leq a_{n+1}$ so

$$
C_{n+1}^{n+1} \subset C_{n+1}^{n} \subset C_{n}^{n} .
$$

If $a$ is big enough, so $a_{*}$ is big enough, this implies that $\Gamma\left(I_{i}\right) \subset \mathcal{L}_{c_{0}}\left(\mathcal{L}_{c^{\prime}}\left(I_{i}\right)\right)$ is deepinside $I_{i-1}$, which contradicts $\left|I_{i-1}\right| /\left|I_{i+1}\right|<v$.

Remark. From the proof of Claim 1 we get the following. Assume that none of the intervals $\mathcal{C}^{n}\left(\hat{\mathcal{L}}_{c^{\prime}}\left(I_{i-1}\right)\right)$ is terminating for $1 \leq n \leq n_{0}$ and that there exists $G_{j} \in \mathbb{G}$ with $G_{j} \subset \mathcal{C}^{n_{0}}\left(\hat{\mathcal{L}}_{c^{\prime}}\left(I_{i-1}\right)\right)$. Then $n_{0}<a$. Now we pull back from time $s_{i-1}$ to time $j_{0}$.

Claim 2. There exist $\lambda_{0} \in(0,1)$ and an interval $K_{0} \supset G_{j_{0}}$ that is well-inside $I^{m(0)}=$ $I_{i-1}$, so that

$$
U_{j_{0}} \subset D_{\lambda_{0} \theta}\left(K_{0}\right)
$$


By Theorem 3.1(a) we know that there exists $\rho>0$ so that $I_{i-1}$ is $\rho$-nice. Since $\left|I_{i-2}\right| /\left|I_{i+1}\right|<v$, Corollary 3.25 implies there exists $\delta>0$ such that $I_{i-1}$ is $\delta$-free. Shrinking $\delta$, if necessary, we can assume that $I_{i-1}$ is $\delta$-nice and $\delta$-free. Let $t<s_{i-1}$ be maximal so that $G_{t} \subset I_{i-1}$. Since $I_{i-1}$ is $\delta$-free we can apply Lemma 4.6 to find $\lambda^{\prime}>0$ so that

$$
U_{t} \subset D_{\lambda^{\prime} \theta}\left(\mathcal{L}_{G_{t}}\left(I_{i-1}\right)\right)
$$

with $(1+2 \delta) \mathcal{L}_{G_{t}}\left(I_{i-1}\right) \subset I_{i-1}$. There are two cases to consider:

Case (i): $k\left(I_{i-1}, \mathbb{G}\right)$ is finite.

Since we are assuming $\left|I_{i-1}\right| /\left|I_{i+1}\right|<v$, Claim 1 tells us that $k\left(I_{i-1}, \mathbb{G}\right)<a$. In this case Proposition 7.1 allows us to control the loss of angle from time $t$ to time $t_{1}^{\prime}$. Since $G_{t_{1}^{\prime}}$ is the first entry of $G_{t_{1}}$ to $I_{i-1}$ we can control the loss of angle from time $t_{1}^{\prime}$ to time $t_{1}$ using Corollary 4.8; this means that we can find an interval $J_{1}$ and constants $\lambda_{1}, \delta_{1}>0$ such that

$$
U_{t_{1}} \subset D_{\lambda_{1} \theta}\left(J_{1}\right)
$$

with $G_{t_{1}} \subset J_{1} \subset\left(1+2 \delta_{1}\right) J_{1} \subset \hat{\mathcal{L}}_{G_{t_{1}}}\left(I_{i-1}\right)$. Let $j_{0} \leq t_{2}<t_{1}$ be maximal so that $\hat{\mathcal{L}}_{G_{t_{2}}}\left(I_{i}\right)$ contains a critical point. If $t_{2}$ is not defined, the proposition follows after one application of Corollary 4.8. If $t_{2}$ is defined let $t_{2}^{\prime}$ with $t_{2}<t_{2}^{\prime} \leq t_{1}$ be minimal such that $G_{t_{2}^{\prime}} \subset I_{i-1}$. If we let $\mathbb{G}=\left\{G_{j}\right\}_{j=t_{2}^{\prime}}^{t_{1}}$, either Case (i) or Case (ii) holds. If Case (i) holds, we repeat the previous argument. See below for the argument in Case (ii). The proposition follows after repeating this argument at most once for each point $c \in \operatorname{Crit}\left(I_{i-1} ;\left\{G_{j}\right\}_{j=j_{0}}^{t_{1}-1}\right)$, so at most $b-1$ times.

Case (ii), $k\left(I_{i-1}, \mathbb{G}\right)=\infty$. By the definition of combinatorial depth, there exist $n \in \mathbb{N}$, $t_{1}^{\prime} \leq j \leq s_{i-1}$ and a point $c \in \operatorname{Crit}\left(I_{i-1} ; \mathbb{G}\right)$, so that $G_{j} \subset \mathcal{C}^{n}\left(\hat{\mathcal{L}}_{c}\left(I_{i-1}\right)\right)$, where $\mathcal{C}^{n}\left(\hat{\mathcal{L}}_{c}\left(I_{i-1}\right)\right)$ is a periodic interval. For each $c \in \operatorname{Crit}\left(I_{i-1} ; \mathbb{G}\right)$, pick $n_{c}$ minimal and $m_{c}$ maximal so that $t_{1}^{\prime} \leq n_{c} \leq m_{c} \leq s_{i-1}$ and $G_{n_{c}}, G_{m_{c}} \subset \hat{\mathcal{L}}_{c}\left(I_{i-1}\right)$. Let $c \in \operatorname{Crit}\left(I_{i-1} ; \mathbb{G}\right)$ be such that $m_{c}$ is maximal. From the remark at the end of Claim 1 we know the following. There exists $m<a$ such that $C^{m}\left(\hat{\mathcal{L}}_{c}\left(I_{i-1}\right)\right)$ is periodic, otherwise $\left|I_{i_{1}}\right| /\left|I_{i+1}\right|>v$. Let $m_{0}$ be minimal such that $C^{m_{0}}\left(\hat{\mathcal{L}}_{c}\left(I_{i-1}\right)\right)$ is a periodic interval. Let $\mathcal{P}=\mathcal{C}^{m_{0}}\left(\hat{\mathcal{L}}_{c}\left(I_{i-1}\right)\right)$. By the choice of $c$ there exists $G_{j} \subset \mathcal{P}$. Even more, since $\mathcal{P}$ is a periodic interval and $G_{k_{l}}$ is the only element of the chain contained in $I_{i}$, there exists only one interval $G_{\hat{t}_{1}} \subset \mathcal{P}$ with $j_{0} \leq \hat{t}_{1}<s_{i-1}$. By the definition of $\hat{t}_{1}$ and the choice of $c$ we can use Proposition 7.1 and Corollary 4.8 to control the loss of angle from time $s_{i-1}$ to time $\hat{t}_{1}$. Thus, there exists an interval $J_{1}$ and constants $\lambda_{1}, \delta_{1}>0$ with $G_{\hat{t}_{1}} \subset J_{1} \subset\left(1+2 \delta_{1}\right) J_{1} \subset \hat{\mathcal{L}}_{c}\left(I_{i-1}\right)$ such that

$$
U_{\hat{t}_{1}} \subset D_{\lambda_{1} \theta}\left(J_{1}\right)
$$

Pick $j_{0} \leq t_{2}<\hat{t}_{1}$ maximal such that $G_{t_{2}} \subset \mathcal{C}^{n}\left(\mathcal{L}_{c^{\prime}}\left(I_{i-1}\right)\right)$ for some $c^{\prime} \in$ $\operatorname{Crit}\left(I_{i-1} ;\left\{G_{j}\right\}_{j=j_{0}}^{\hat{t}_{1}}\right)$ and $\mathcal{C}^{n}\left(\mathcal{L}_{c^{\prime}}\left(I_{i-1}\right)\right)$ terminating; if $t_{2}$ is not defined let $t_{2}=j_{0}$. Let $\hat{t}_{2}<\hat{t}_{1}$ be maximal such that $G_{\hat{t}_{2}} \subset \hat{\mathcal{L}}_{c^{\prime}}\left(I_{i-1}\right)$. Proposition 7.1 and Corollary 4.8 allow us to control the loss of angle from time $\hat{t}_{1}$ to time $t_{2}$. The proof follows repeating the previous argument at most once for each critical point of $f$, so at most $b$ times. $\checkmark$ 
Let us continue with the proof of the proposition. Now that we have pulled back to time $j_{0}$ we pull back to the desired time $s_{i}^{\prime}=j_{0}-k_{*}$. By the choice of $j_{0}$ we can restrict all but a bounded number of these pullbacks to monotone branches of return maps to intervals in the principal nest. See Fig. 16 for an idea of how this will be done. Let us first define the following times; let $\tilde{m}(1)<\tilde{m}(2)<\cdots<\tilde{m}(k)$ be the levels of the principal nest such that there exists a turning point $c$ of $R_{I^{\tilde{m}(i)-1}}$ such that $R_{I^{\tilde{m}(i)-1}}(c) \notin I^{\tilde{m}(i)}$, $i=1,2, \ldots k$. Set $\tilde{m}(0)=0$, so that $I^{\tilde{m}(0)}=I^{0}=I_{i-1}$. Notice that the terminating interval $\tilde{I}$ is equal to $I^{\tilde{m}(k)}$ and that the return time of $c_{0}$ to $\tilde{I}$ is $r$. Let $j_{0}^{\prime}<j_{0}$ be minimal so that for all $t, j_{0}^{\prime} \leq t<j_{0}$, we have that if $G_{t} \subset I^{\tilde{m}(0)}$, then $G_{t} \subset I^{\tilde{m}(0)+1} \backslash I^{\tilde{m}(1)}$. Define $j_{1}<j_{0}^{\prime}$ to be maximal so that $G_{j_{1}} \subset I^{\tilde{m}(1)}$. Similarly, define $j_{1}^{\prime}>j_{2}>j_{2}^{\prime}>\cdots j_{k}$.

If $\tilde{m}(1)=\tilde{m}(0)+1$ or $\tilde{m}(0)+2$, then by applying Corollary 4.8 once or twice there exist $\lambda_{1} \in(0,1)$, and an interval $K_{1}$ that is well-inside $I^{\tilde{m}(1)}$ such that

$$
U_{j_{1}} \subset D_{\lambda_{1} \theta}\left(K_{1}\right)
$$

so suppose that $\tilde{m}(1)>\tilde{m}(0)+2$. Let $q_{0}$ be such that $f^{q_{0}}\left|I^{\tilde{m}(0)+1}=R_{I^{\tilde{m}(0)}}\right| I^{\tilde{m}(0)+1}$. Since $\left|I^{\tilde{m}(0)+1}\right| /\left|I^{\tilde{m}(0)+2}\right|<\nu$, and $I^{\tilde{m}(0)+1}$ is well-inside $I^{\tilde{m}(0)}$, Lemma 3.10 implies that there exists $\kappa_{0}>0$ and $C$ such that for any critical point $c$ of $R_{I^{\tilde{m}(0)+1}} \mid I^{\tilde{m}(0)+2}$,

$$
\left|f^{q_{0}}(c)-c\right| \geq \kappa_{0}\left|I^{\tilde{m}(0)}\right| \text {, and }\left|D\left(f^{q_{0}}\right)(x)\right| \leq C \text { for all } x \in I^{\tilde{m}(0)+2} .
$$

Since $f^{q_{0}}$ is a first return map, by Corollary 4.8 , there exist $\lambda^{\prime} \in(0,1)$ and an interval $K^{\prime}$ which is well-inside $I^{\tilde{m}(0)+1}$ such that

$$
U_{j_{0}-q_{0}} \subset D_{\lambda^{\prime} \theta}\left(K^{\prime}\right) .
$$

We have that $G_{j_{0}-2 q_{0}} \subset I^{\tilde{m}(0)+2} \backslash I^{\tilde{m}(1)}$. Let $c_{1}$ be the turning point of $f^{q_{0}}$ on the boundary of the monotone branch of $f^{q_{0}}$ containing $G_{j_{0}-2 q_{0}}$. Let $\mathcal{Y}$ denote this monotone branch. We will now pull back to get the base of the Poincare disk inside of $\mathcal{Y}$. Since $c_{1} \in I^{\tilde{m}(1)}$, $G_{j_{0}-q_{0}}$ does not contain $f^{q_{0}}\left(c_{1}\right)$. Let $c^{\prime}$ be the critical point of $f$ with the property that $f^{k}\left(c_{1}\right)=c^{\prime}$ with $0 \leq k<q_{0}$ maximal. Let $\alpha$ be the fixed point of $f^{q_{0}}$ closest to $c_{0}$ and let $\alpha^{\prime} \in \operatorname{Comp}_{c^{\prime}} f^{q_{0}-k}\left(I^{\tilde{m}(0)+1}\right)$ be the preimage of $\alpha$ that lies on the opposite side of $c^{\prime}$ to $G_{j_{0}-2 q_{0}+k}$. By Lemma 3.10, $\left|c_{1}-\alpha\right|$ is comparable to $\left|I^{\tilde{m}(0)}\right|$, so there exists an interval $J_{0} \subset\left(c_{1}, \alpha\right)$ such that $\left|J_{0}\right|>\delta_{0}\left|I^{\tilde{m}(0)}\right|$ and $J_{0}$ does not contain any critical values of $f^{q_{0}}$. Let $J_{0}^{\prime}$ be the pullback of $J_{0}$ in $\left(c^{\prime}, \alpha^{\prime}\right)$, and let $J_{1}^{\prime}$ be the pullback by $f^{-\left(q_{0}-k\right)}$ of the connected component of $I^{m(0)+1} \backslash I^{m(0)+2}$ on the same side of $c_{1}$ as $G_{j_{0}-q_{0}}$, so that the convex hull of $J_{0}^{\prime} \cup J_{1}^{\prime}$ contains $G_{j_{0}-2 q_{0}+k}$. There exists $\delta_{0}^{\prime}>0$ such that $\left|J_{0}^{\prime}\right|,\left|J_{1}^{\prime}\right|>\delta_{0}^{\prime}\left|\mathcal{L}_{c^{\prime}}\left(I^{\tilde{m}(0)+1}\right)\right|$. Let $K^{\prime \prime}$ be the convex hull of $J_{0}^{\prime} \cup J_{1}^{\prime}$. There exists $\delta^{\prime \prime}>0$ and an interval $K_{0}^{\prime}$ which is well-inside $K^{\prime \prime}$ such that $\left|K_{0}^{\prime}\right|$ is comparable to $\left|\mathcal{L}_{c^{\prime}}\left(I^{m(0)}\right)\right|$ and $\left(1-2 \delta^{\prime \prime}\right) K_{0}^{\prime} \backslash\left(1-2 \delta^{\prime \prime}\right)^{-1} K_{0}^{\prime}$ contains no critical values of $f^{k}$. Since $G_{j_{0}-2 q_{0}+k}$ does not contain $c^{\prime}$ by Lemma 4.4 , there exists $\lambda_{0}^{\prime} \in(0,1)$

$$
U_{j_{0}-2 q_{0}+k} \subset D_{\lambda_{0}^{\prime} \theta}\left(K_{0}^{\prime}\right)
$$

Since $K_{0}^{\prime}$ is $\delta^{\prime \prime}$-free we can apply by Lemma 4.6 to find $\lambda_{1} \in(0,1)$ and an interval $K_{1}$ that is well-inside $\mathcal{Y}$, such that $G_{j_{0}-2 q_{0}} \subset K_{1}$ and

$$
U_{j_{0}-2 q_{0}} \subset D_{\lambda_{1} \theta}\left(K_{1}\right) \text {. }
$$


Now, we use an argument similar to the one in the proof of Claim 6 of Proposition 6.3 to pull back to a Poincaré disk based in $I^{\tilde{m}(1)}$. By Proposition 6.1 and at most one application of Corollary 4.8, we have that there exists $\lambda_{2} \in(0,1)$ such that

$$
U_{j_{0}^{\prime}} \subset D_{\lambda_{2} \theta}\left(\mathcal{Y}^{\prime}\right)
$$

where $\mathcal{Y}^{\prime}$ is an outermost monotone branch of $f^{q_{0}} \mid I_{\tilde{m}(0)+1}^{\tilde{m}(1)}$. So we have that there exists $\lambda_{3} \in(0,1)$ and an interval $K_{3}$ that is well-inside $I^{\tilde{m}(1)}$ such that

$$
U_{j_{1}} \subset D_{\lambda_{3}}\left(K_{3}\right) \text {. }
$$

Let $q_{1}$ be the return time of $I^{\tilde{m}(1)+1}$ to $I^{\tilde{m}(1)}$. By Corollary 4.8, we have that there exists $\lambda_{4} \in(0,1)$ and an interval $K_{4}$ which is well-inside $I^{\tilde{m}(1)+1}$ such that

$$
U_{j_{1}-q_{1}} \subset D_{\lambda_{4} \theta}\left(K_{4}\right) .
$$

Observe that $G_{j_{1}-q_{1}} \subset I^{m(1)+1} \backslash I^{m(1)+2}$.

Since $I^{m(0)}=I_{i-1}$ is $\delta$-nice, there exists $\delta_{1}>0$ such that $I^{m(1)+1}$ is $\delta_{1}$-nice. If $j \in\{0, \ldots, k-1\}$ is such that there exists $j^{\prime} \in\{0, \ldots, l-1\}$ such that $\tilde{m}(j)=m\left(j^{\prime}\right)$, then by Lemma 3.8 there exists $\delta_{1}^{\prime}>0$ such that $I^{\tilde{m}(j)+1}$ is $\delta_{1}^{\prime}$-nice. If this is not the case, then $\mathcal{L}_{c_{0}}\left(I^{\tilde{m}(j)}\right)$ is well-inside $I^{\tilde{m}(j)}$, since there exist two turning points $c_{1}, c_{2}$, critical points of $f^{q_{0}}$, such that $\mathcal{L}_{c_{0}}\left(I^{\tilde{m}(j)}\right) \subset\left(c_{1}, c_{2}\right)$, and by Lemma 3.10, the interval $\left(c_{1}, c_{2}\right)$ is well-inside $I^{\tilde{m}(j)}$. Shrinking $\delta_{1}$ if necessary, $I^{\tilde{m}(j)+1}$ is $\delta_{1}$-nice for all $j=0, \ldots, k$. Since $\left|I_{i-1}\right| /\left|I_{i}\right|<v, k$ is bounded. We complete the proof the proposition by repeating the previous argument, to pull back from time $\tilde{m}(0)$ to $\tilde{m}(1), k-1$ times.

Combinatorial Remark 2. Note that there exist at most two intervals $G_{j}$ with $s_{i}^{\prime} \leq j \leq$ $s_{i-1}$ and $G_{j} \subset I_{i}^{\infty}$, and one of them is $G_{s_{i}^{\prime}}$. Thus, there exists at most one interval $G_{j}$ with $s_{i}^{\prime} \leq j \leq s_{i-1}$ with $G_{j} \subset I_{i+1}$.

In the next proposition, we will deal with the first step of the pullback when $I_{i-1}$ is terminating, see Fig. 14.

Proposition 8.3. For each $v \geq 1$ there exist $\mu=\mu(v) \in(0,1)$ and $\delta>0$, and for each $\theta \in(0, \pi)$ there exists $\varepsilon>0$ such that the following holds. Assume that $I_{i-1}$ is terminating with $\left|I_{i-1}\right|<\varepsilon$ and that

$$
\frac{\left|I_{i-2}\right|}{\left|I_{i+1}\right|}<v .
$$

Let $\left\{G_{j}\right\}_{j=0}^{s}$ be a disjoint chain with $G_{s}=I_{n}, G_{0} \subset I_{n}$ for some $n>i$ and $G_{0} \cap \omega\left(c_{0}\right) \neq$ $\emptyset$. Suppose $s_{i-1} \leq s$ is such that at least three of the intervals $G_{0}, G_{1}, \ldots G_{s_{i-1}}$ are contained in $I_{i+1}$. Then there exists $r_{i-1}<s_{i-1}$ with $G_{r_{i-1}} \subset \mathcal{R}\left(I_{i-1}\right)$ such that if

$$
U_{s_{i-1}}=D_{\theta}\left(I_{i-1}\right) \cap \mathbb{C}_{G_{s_{i-1}}} \text { and } U_{j}=\operatorname{Comp}_{G_{j}} f^{-\left(s_{i-1}-j\right)}\left(U_{s_{i-1}}\right),
$$

then there exists an interval $K$ so that

$$
U_{r_{i-1}} \subset D_{\mu \theta}(K),
$$

and $(1+2 \delta) K \subset \mathcal{R}\left(I_{i-1}\right)$. Moreover, there are at most three times $j$ with $r_{i-1} \leq j \leq s_{i-1}$ and $G_{j} \subset I_{i+1}$. 
Proof. Let $r$ be the period of $I_{i-1}^{\infty}$. Since $I_{i-1}$ is terminating, $I_{i-1}^{\infty}$ is a periodic interval, and $\mathcal{R}\left(I_{i-1}\right)$ is bounded between $\alpha_{i-1}$ and $\tau\left(\alpha_{i-1}\right)$, where $\alpha_{i-1}$ is the orientation reversing fixed point of $R_{I_{i-1}}$ closest to $c_{0}$. To be definite let $Y_{-1} \neq Y_{0}$ be the other component of $I_{i-1}^{\infty} \backslash\left(f^{r} \mid I_{i-1}^{\infty}\right)^{-1}(\alpha)$ that contains $\alpha_{i-1}$ in its boundary, let $Y_{-a}$ be the component containing $\beta$, the fixed point of $R_{I_{i-1}^{\infty}}$ contained in $\partial I_{i-1}^{\infty}$, and $Y_{a}$ be the component containing $\tau(\beta)$ in its boundary. For simplicity, we will denote $\alpha_{i-1}$ by $\alpha$.

Step 1: Pulling back to $I_{i-1}^{\infty}$. We define a time $s_{i-1}^{\infty}$ with $0 \leq s_{i-1}^{\infty}<s_{i-1}$ so that the following holds. There exist $\lambda_{0} \in(0,1)$ and an interval $K_{0}$ well-inside $I_{i-1}^{\infty}$ so that

$$
U_{s_{i-1}^{\infty}} \subset D_{\lambda_{0} \theta}\left(K_{0}\right) .
$$

Since $I_{i-1}$ is terminating, only one component of its return domain intersects $\omega\left(c_{0}\right)$, so $G_{s_{i-1}} \subset I_{i-1}^{\infty}$. Let us first pull back by one iterate of $f^{r}$. Since $\left|I_{i-2}\right| /\left|I_{i+1}\right|<v$ we can apply Corollary 3.25 to find $\rho>0$ so that $I_{i-1}$ is $\rho$-free. Notice that since $I_{i-1}$ is terminating, all critical points of $f^{r} \mid I_{i-1}^{1}$ are contained in $I_{i-1}^{\infty}$. Let $0<j \leq r$ be minimal so that $\mathcal{L}_{f^{r-j}\left(c_{0}\right)}\left(I_{i-1}\right)$ contains a turning point $c^{\prime}$ of $f$ and $c^{\prime} \notin G_{s_{i-1}-j}$. Then $G_{s_{i-1}-j}$ is contained in an interval bounded on one side by a preimage $\hat{\alpha}$ of $\alpha$ (such that $\left.f^{r-j}(\hat{\alpha})=\alpha\right)$ and on the other either by $c^{\prime}$ or by a boundary point of $\mathcal{L}_{f^{r-j}\left(c_{0}\right)}\left(I_{i-1}\right)$. Let $\alpha^{\prime}$ be the symmetric point to $\hat{\alpha}$ with respect to $c^{\prime}$. Since $I_{i-1}$ is $\rho$-free we can apply Lemma 3.10(2) to conclude that $\left|c^{\prime}-\alpha^{\prime}\right|$ is comparable to $\left|\mathcal{L}_{f^{r-j}\left(c_{0}\right)}\left(I_{i-1}\right)\right|$. As in the previous lemma, this means that there exist an interval $K^{\prime}, \lambda^{\prime} \in(0,1)$ and $\delta^{\prime}>0$ such that

$$
U_{s_{i-1}-j} \subset D_{\lambda^{\prime} \theta}\left(K^{\prime}\right),
$$

where $K^{\prime}$ is an interval that is bounded on one side by a boundary point $x$ of $\mathcal{L}_{f^{r-j}\left(c_{0}\right)}\left(I_{i-1}\right)$ and on the other by a point $x^{\prime} \in\left(c^{\prime}-\delta^{\prime}\left|\mathcal{L}_{f^{r-j}\left(c_{0}\right)}\left(I_{i-1}\right)\right|, c^{\prime}+\right.$ $\left.\delta^{\prime}\left|\mathcal{L}_{f^{r-j}\left(c_{0}\right)}\left(I_{i-1}\right)\right|\right)$, on the opposite side of $c^{\prime}$ as $x$. Additionally, we can assume $K^{\prime}$ has the property that $\left(1+\delta^{\prime}\right) K^{\prime} \backslash\left(1+\delta^{\prime}\right)^{-1} K^{\prime}$ is disjoint from $\cup\left\{f(c), f^{2}(c), \ldots f^{r}(c)\right\}$, where the union is taken over all critical points $c$ of $f$. Replacing $\mathcal{L}_{f^{r-j}\left(c_{0}\right)}\left(I_{i-1}\right)$ by $K^{\prime}$ and repeating this process at most once for each critical point of $f$, we have that there exists an interval $K_{1} \subset I_{i-1}^{1}, \lambda_{1} \in(0,1)$ and $\delta_{1}>0$ such that

$$
U_{s_{i-1}-r} \subset D_{\lambda_{1} \theta}\left(K_{1}\right),
$$

where $K_{1}$ is of one of the following types.

(a) Assume $G_{s_{i-1}-r}$ is contained in one of the two outermost monotone branches of $f^{r} \mid I_{i-1}^{1}$. Then $K_{1}$ is bounded between a boundary point $x_{1}$ of $\mathcal{L}_{c_{0}}\left(I_{i-1}\right)$ and a point $x_{2} \in\left(c_{1}-\delta_{1}\left|I_{i-1}^{\infty}\right|, c_{1}+\delta_{1}\left|I_{i-1}^{\infty}\right|\right)$ on the opposite side of $c_{1}$ as $x_{1}$ where $c_{1}$ is an outermost turning point of $f^{r}$;

(b) Assume $G_{s_{i-1}-r}$ is contained in $I_{i-1}^{\infty}$ minus the two outermost monotone branches of $f^{r} \mid I_{i-1}^{1}$. Then there exist turning points $c_{1}<c_{2}$ of $f^{r}$ and points $x_{1} \in\left(c_{1}-\right.$ $\left.\delta_{1}\left|I_{i-1}^{\infty}\right|, c_{1}\right) \subset I_{i-1}^{\infty}$ and $x_{2} \in\left(c_{2}, c^{\prime}+\delta_{1}\left|I_{i-1}^{\infty}\right|\right) \subset I_{i-1}^{\infty}$ such that $K_{1}=\left(x_{1}, x_{2}\right)$ is well-inside $I_{i-1}^{\infty}$;

(c) Assume that $G_{s_{i-1}-r}$ contains one of the turning points from the two outermost monotone branches of $f^{r} \mid I_{i-1}^{1}$. Then, $K_{1}$ is bounded on one side by a boundary point $x_{1}$ of $\mathcal{L}_{c_{0}}\left(I_{i-1}\right)$ and on the other side by a point $x_{2} \in\left(c_{0}-\delta_{1}\left|I_{i-1}^{\infty}, c_{0}+\delta_{1}\right| I_{i-1}^{\infty} \mid\right)$ on the opposite side of $c_{0}$ as $x_{1}$. 
Suppose first that (a) holds. Let $\mathcal{Y}$ denote the outermost monotone branch of $f^{r} \mid I_{i-1}^{1}$ containing $G_{s_{i-1}-r}$ and let $c$ be the turning point on its boundary. If $\mathcal{Y}$ is the orientation reversing branch, pulling back under one more iterate of $f^{r}$, as in the proof of Claim 6 of Proposition 6.3, we obtain an interval $K_{2}$ that is well-inside $I_{i-1}^{\infty}$, and $\lambda_{2} \in(0,1)$ such that

$$
U_{s_{i-1}-2 r} \subset D_{\lambda_{2} \theta}\left(K_{2}\right),
$$

and $s_{i-1}^{\infty}=s_{i-1}-2 r$. We know that $s_{i-1}-2 r>0$, since at least two of $G_{0}, G_{1}, \ldots G_{s_{i-1}-1}$ are contained in $I_{i+1}$. Even more, the only interval in $G_{s_{i-1}^{\infty}}, G_{s_{i-1}^{\infty}+1}$, $\ldots, G_{s_{i-1}-1}$ that could be contained in $I_{i+1}$ is $G_{s_{i-1}^{\infty}}$. Now suppose that $\mathcal{Y}$ is the orientation preserving branch. Let $q \geq 1$ be maximal so that $G_{s_{i-1}-q r} \subset \mathcal{Y}$ and for $t, 1 \leq t \leq q$, $G_{s_{i-1}-t r} \subset \mathcal{Y}$. If $q=1$, then either

- there exists an interval $K_{2}$ which is well-inside $I_{i-1}^{\infty}$, and $\lambda_{2} \in(0,1)$ such that

$$
U_{s_{i-1}-2 r}(z) \subset D_{\lambda_{2} \theta}\left(K_{2}\right),
$$

and $s_{i-1}^{\infty}=s_{i-1}-2 r$ or

- $G_{s_{i-1}-2 r}$ is contained in the outermost orientation reversing branch of $f^{r} \mid I_{i-1}^{1}$, $s_{i-1}-3 r>0$ and, as above, we have that there exists an interval $K_{2}$ which is well-inside $I_{i-1}^{\infty}$, and $\lambda_{2} \in(0,1)$ such that

$$
U_{s_{i-1}-3 r}(z) \subset D_{\lambda_{2} \theta}\left(K_{2}\right) .
$$

So suppose that $q>1$. Let $c_{-1}$ be the preimage of $c$ under $f^{r}$ contained in $\mathcal{Y}$. By Lemma 3.10, the interval $\left(c_{-1}, c\right)$ has length comparable to $I_{i-1}$, and by Lemmas 3.9 and 3.7 there exists a fundamental domain for $f^{r} \mid \mathcal{Y}$ contained in $I_{i-1}^{1} \backslash I_{i-1}^{\infty}$ of size comparable to $I_{i-1}$. So by Proposition 6.1, there exists $\lambda_{2}>0$ such that

$$
U_{s_{i-1}-q r} \subset D_{\lambda_{2} \theta}(\mathcal{Y}) \text {. }
$$

Arguing as in the case when $q=1$, we have that $s_{i-1}^{\infty}=s_{i-1}-(q+1) r$ or $s_{i-1}^{\infty}=$ $s_{i-1}-(q+2) r$, and there exist an interval $K_{3}$ which is well-inside $I_{i-1}^{\infty}$ and $\lambda_{3} \in(0,1)$ such that

$$
U_{s_{i-1}^{\infty}} \subset D_{\lambda_{3} \theta}\left(K_{3}\right)
$$

Now, suppose that either (b) or (c) holds. If (b) holds, then $s_{i-1}-r=s_{i-1}^{\infty}$ and we are done with this step. If (c) holds, applying the argument at the start of Step 1 to pull back once under $f^{r}$, we have that either (a) or (b) holds, since the chain $\left\{G_{j}\right\}_{j=0}^{s_{i-1}}$ is disjoint. In any case, we have that there exists an interval $K_{0}$ which is well-inside $I_{i-1}^{\infty}$, and $\lambda_{0} \in(0,1)$ such that

$$
U_{s_{i-1}^{\infty}} \subset D_{\lambda_{0} \theta}\left(K_{0}\right)
$$

Combinatorial Remark 3. Note that there exist at most two intervals $G_{j} \subset \mathcal{R}\left(I_{i-1}\right)$ with $s_{i-1}^{\infty} \leq j \leq s_{i-1}$, so there exists at most one interval $G_{j} \subset I_{i+1}$ with $s_{i-1}^{\infty} \leq j \leq$ $s_{i-1}$. 
Since $c$ is a critical point of $f^{r}$, we have that $|c-\alpha|$ is comparable to $\left|I_{i-1}^{\infty}\right|$, so we can choose the previous constants so that there exists $\delta_{0}>0$ such that $\left(1+\delta_{0}\right) K_{0} \backslash(1+$ $\left.2 \delta_{0}\right)^{-1} K_{0}$ does not contain $\alpha$. From now on, we will consider pullbacks by $f^{r} \mid I_{i-1}^{\infty}$.

Step 2: Pulling back to a $\hat{\rho}$-nice interval. We define a time $t$, with $0<t<s_{i-1}^{\infty}$ with the following properties. There exist a $\hat{\rho}$-nice interval $X$, for $\hat{\rho}>0$ universal, and $\lambda^{\prime \prime}>0$ so that $G_{t} \subset X$ and $U_{t} \subset D_{\lambda^{\prime \prime} \theta}\left(K^{\prime \prime}\right)$, for an interval $K^{\prime \prime}$ that is well-inside $X$. We will make use of the intervals $Y_{j}$ defined in Sect. 2 to find $X$. Remember $Y_{0}=\mathcal{R}\left(I_{i-1}\right) ; Y_{-a}$ contains $\beta$, the fixed point of $f^{r}$ in the boundary of $I_{i-1}^{\infty}$; and $Y_{a}$ contains $\tau(\beta)$.

Step 2(a). First, suppose that $\alpha \notin K_{0}$, then pulling back once under $R_{I_{i-1}^{\infty}}$, we can find an interval $K_{1}^{\prime}$, and a $\lambda_{1}^{\prime} \in(0,1)$ such that $U_{s_{i-1}^{\infty}-r} \subset D_{\lambda_{1}^{\prime} \theta}\left(K_{1}^{\prime}\right)$ where $K_{1}^{\prime}$ is well-inside $Y_{j}$ for some $j$. If $j=0$ we set $r_{i-1}=s_{i-1}^{\infty}-r$ and we are done with the proof of this proposition in this case. Note that if $j=0$ there are at most two times $j$ with $r_{i-1} \leq j \leq s_{i-1}$ and $G_{j} \subset I_{i+1}$. In the remainder of this step, we will assume that $j \neq 0$. Note that this implies that $s_{i-1}^{\infty}-r>0$.

Case 1: If $j \notin\{a,-a, 1\}$, then by Lemma 3.22 we know that $Y_{j}$ is $\hat{\rho}$-nice, so we set $X=Y_{j}$ and $t=s_{i-1}^{\infty}-r$. Note that in this case there exists no $G_{j}$ with $G_{j} \subset \mathcal{R}\left(I_{i-1}\right)$ and $t \leq j \leq s_{i-1}^{\infty}-r$.

Case 2: If $j=a$, then $s_{i-1}^{\infty}-2 r>0$ and after one more pull back by $f^{r}$ we can find an interval $K_{2}^{\prime}$ and a $\lambda_{2}^{\prime} \in(0,1)$ such that $U_{s_{i-1}^{\infty}-2 r} \subset D_{\lambda_{2}^{\prime} \theta}\left(K_{2}^{\prime}\right)$ where $K_{2}^{\prime}$ is well-inside $Y_{j^{\prime}}$. Since $K_{1}^{\prime} \subset Y_{a}$, we have that $j^{\prime} \notin\{a,-a\}$. If $j^{\prime} \notin\{0,1\}$ we set $t=s_{i-1}^{\infty}-2 r>0$ and $X=Y_{j^{\prime}}$ and go to Step 3. Note that if $j^{\prime} \notin\{0,1\}$ there exists no $G_{j} \subset \mathcal{R}\left(I_{i-1}\right)$ with $t \leq j \leq s_{i-1}^{\infty}-r$. If $j^{\prime}=0$, we set $r_{i-1}=s_{i-1}^{\infty}-2 r$ and we are done with the proof of the proposition. If $j^{\prime}=-1$ we go to Case 4 .

Case 3: If $j=-a$, let $q$ be such that $q r=s_{i-1}^{\infty}-r$, and let $q^{\prime} \leq q$ be minimal so that for $k, q^{\prime} \leq k \leq q, G_{k r} \subset Y_{-a}$. Since $\left.f^{r}\right|_{Y_{-a}}$ is monotone we can apply Proposition 6.1 to control the loss of angle until time $q^{\prime} r$. Then after pulling back once more, we can find an interval $K_{2}^{\prime}$ and a $\lambda_{2}^{\prime} \in(0,1)$ such that $U_{\left(q^{\prime}-1\right) r}(z) \subset D_{\lambda_{2}^{\prime} \theta}\left(K_{2}^{\prime}\right)$. Let $Y_{j^{\prime}}$ be the puzzle piece containing $K_{2}^{\prime}$, then $K_{2}^{\prime}$ is well-inside $Y_{j^{\prime}}$. If $j^{\prime}=0$ we set $r_{i-1}=\left(q^{\prime}-1\right) r$ and we are done with the proof of the proposition. If $j^{\prime} \notin\{-a, 1\}$, then $Y_{j^{\prime}}$ is $\delta^{\prime}$-nice for some $\delta^{\prime}>0$ so we set $X=Y_{j^{\prime}}$ and $t=\left(q^{\prime}-1\right) r$. Note that if $j^{\prime} \notin\{-a, 0,1\}$ there exists no $G_{j} \subset \mathcal{R}\left(I_{i-1}\right)$ with $t \leq j \leq s_{i-1}^{\infty}-r$. If $j^{\prime}=a$, go to Case 2 , and if $j^{\prime}=1$ go to Case 4.

Case 4: Suppose that $j=-1$. If $G_{s_{i-1}^{\infty}-2 r}$ is contained $Y_{0}$, we set $r_{i-1}=s_{i-1}^{\infty}-2 r$ and we are done with the proof of the proposition; if $G_{s_{i-1}^{\infty}-2 r} \subset Y_{j^{\prime}}$ with $j^{\prime} \notin\{a,-a\}$ we set $t=s_{i-1}^{\infty}-2 r$ and $X=Y_{j^{\prime}}$; if $G_{s_{i-1}^{\infty}-r} \subset Y_{j^{\prime}}$ with $j^{\prime} \in\{a,-a\}$, we complete the proof of this step using the appropriate previous case. Observe that if we go from Case 2 to Case 4 and back to Case 2 or from Case 3 to Case 4 and back to Case 3, then by Lemma 3.22 we can set $X=\mathcal{L}_{G_{t}}\left(Y_{a}\right)$ or $X=\mathcal{L}_{G_{t}}\left(Y_{-a}\right)$, for a suitable time $t$.

Combinatorial Remark 4. If after applying Step 2(a) we have not proved the proposition, then there exists no $G_{j} \subset \mathcal{R}\left(I_{i-1}\right)$ with $t \leq j \leq s_{i-1}^{\infty}-r$. This means that there exists at most one $G_{j} \subset I_{i+1}$ with $t \leq j \leq s_{i-1}$.

Step 2(b). Suppose that $\alpha \in\left(1+\delta_{0}\right) K_{0}$. Recall that $K_{0} \supset G_{s_{i-1}^{\infty}}$. First, as in Step 1 of this proposition, pulling back by one iterate of $f^{r}$ we have that there exists an interval $K_{1}^{\prime}$ which is well-inside $I_{i-1}^{\infty}$ and $\lambda_{1}^{\prime} \in(0,1)$ such that 


$$
U_{s_{i-1}^{\infty}-r} \subset D_{\lambda_{1}^{\prime} \theta}\left(K_{1}^{\prime}\right) \text {. }
$$

If $G_{s_{i-1}^{\infty}-r}$ is not contained in $\tilde{\mathcal{Y}}_{\alpha}$ we are in Step 2(a). Otherwise, $G_{s_{i-1}^{\infty}-r} \subset \tilde{\mathcal{Y}}_{\alpha}$ and we can ensure that the pullback of $K_{1}^{\prime}$ under $f^{r} \mid \tilde{\mathcal{Y}}_{\alpha}$ is well-inside $\tilde{\mathcal{Y}}_{\alpha}$. Let $p$ be such that $p r=s_{i-1}^{\infty}-r$, and let $p^{\prime} \leq p$ be minimal so that for $j, p^{\prime} \leq j \leq p, G_{j r} \subset \tilde{\mathcal{Y}}_{\alpha}$. We have that $\tilde{\mathcal{Y}}_{\alpha}$ is bounded by two turning points, so by Lemma 3.10 we can find fundamental domains for the map from this monotone branch to itself of size comparable to $\left|I_{i-1}^{\infty}\right|$. By Proposition 6.1 we can control the loss of angle until time $p^{\prime} r$. Observe that for $p^{\prime} \leq k \leq p$ only $G_{p r}$ could be in $I_{i+1}$. Let $j_{1}$ be such that $G_{p^{\prime} r-r} \subset Y_{j_{1}}$. Since $G_{p^{\prime} r-r}$ is not contained in $Y_{0} \cup Y_{-1}$ we are in Step (2a).

Combinatorial Remark 5. If after applying Steps 2(a) and 2(b) we have not proved the proposition, then there exists no $G_{j} \subset \mathcal{R}\left(I_{i-1}\right)$ with $t \leq j \leq s_{i-1}^{\infty}-r$. This means that between time $s_{i-1}$ and time $t$, we can have at most one visit to $I_{i+1}$. Even more, if $\mathcal{R}\left(I_{i-1}\right)$ is non-periodic, there exists at most two intervals $G_{j} \subset I_{i}$ with $t \leq j \leq s_{i-1}^{\infty}$.

Step 3: Pulling back to $\mathcal{R}\left(I_{i-1}\right)$. Let $r_{i-1}<t$ be maximal with $G_{r_{i-1}} \subset \mathcal{R}\left(I_{i-1}\right)$. Let $t^{\prime}$, $r_{i-1}<t^{\prime}<t$, be minimal with the property that $G_{t^{\prime}} \subset X$. Since $G_{r_{i-1}}$ is the only element of the chain contained in $\mathcal{R}\left(I_{i-1}\right)$ we know that for each $c \in \operatorname{Crit}\left(X ;\left\{G_{j}\right\}_{j=r_{i-1}}^{t}\right)$ there exists at most one interval $G_{j}$ such that $G_{j} \subset \hat{\mathcal{L}}_{c}\left(\mathcal{R}\left(I_{i-1}\right)\right)$. Let $t_{1}<t$ be maximal so that $G_{t_{1}} \subset \hat{\mathcal{L}}_{c}\left(\mathcal{R}\left(I_{i-1}\right)\right)$. If $t_{1}$ is not defined let $t_{1}=r_{i-1}$. Let $t_{1}^{\prime}>t_{1}$ be minimal such that $G_{t_{1}^{\prime}} \subset X$ and let $\mathbb{G}=\left\{G_{j}\right\}_{j=t_{1}^{\prime}}^{t}$. There are two cases to consider:

(i) $k(X, \mathbb{G})<\infty$ or

(ii) $k(X, \mathbb{G})=\infty$.

For each $c \in \operatorname{Crit}(X ; \mathbb{G})$ pick $t_{1}^{\prime} \leq n_{c}<t$ minimal such that $G_{n_{c}} \subset \mathcal{L}_{c}(X)$ and $t_{1}^{\prime} \leq m_{c} \leq t$ maximal such that $G_{m_{c}} \subset \mathcal{L}_{c}(X)$.

Claim. If $k(X, \mathbb{G})$ is finite, there exists $a=a(v)>0$ such that $k(X, \mathbb{G})<a$.

The proof of this claim is analogous to the proof of Claim 1 of Proposition 8.2; one only needs to substitute $I_{i-1}$ by $X$ and $I_{i}$ by $\mathcal{R}\left(I_{i-1}\right)$. Following the proof we get that if $a$ is large enough and $c^{\prime}=c_{0}$, then $\left|\mathcal{R}\left(I_{i-1}\right)\right| /\left|I_{i+1}\right|>v$. If $a$ is large enough and $c^{\prime} \neq c_{0}$ we get that $\left|\mathcal{R}\left(I_{i-1}\right)\right| /\left|\Gamma\left(\mathcal{R}\left(I_{i-1}\right)\right)\right|>v \checkmark$

Case (i). Since we are assuming $\left|\mathcal{R}\left(I_{i-1}\right)\right| /\left|I_{i+1}\right|<v$ the claim tells us that $k(X, \mathbb{G})<a$. In this case the proposition follows arguing as in Case (i) of Proposition 8.2, substituting $j_{0}$ by $r_{i-1}$ and $I_{i-1}$ by $X$ in the proof.

Case (ii). In this case the proposition follows arguing as in Case (ii) of Proposition 8.2, substituting $j_{0}$ by $r_{i-1}, s_{i-1}$ by $t, I_{i-1}$ by $X$ and $I_{i}$ by $\mathcal{R}\left(I_{i-1}\right)$ in the proof.

From Combinatorial Remark 5 we get that at most three intervals in the chain $\left\{G_{j}\right\}_{j=r_{i-1}}^{s_{i-1}}$ are contained in $I_{i+1}$ and the proposition follows. Observe that if exactly three of these intervals are contained in $I_{i-1}, G_{s_{i-1}}$ and $G_{r_{i-1}}$ are both contained in $I_{i+1}$. Moreover, if $\mathcal{R}\left(I_{i-1}\right)$ is non-periodic there exists at most three intervals $G_{j} \subset I_{i}$ with $r_{i-1} \leq j \leq s_{i-1}^{\infty}$. If there are exactly three of these intervals contained in $I_{i}, G_{s_{i-1}}$ and $G_{r_{i-1}}$ are both contained in $I_{i}$. 
Combinatorial Remark 6. It is important to observe that in the proof of Proposition 8.3 we do not have control on the number of visits to $I_{i}$ if $\mathcal{R}\left(I_{i-1}\right)$ is periodic. However, if $I_{i} \neq \mathcal{R}\left(I_{i-1}\right)$ we can prove the proposition assuming that at least three of the intervals $G_{0}, \ldots, G_{s_{i-1}}$ are contained in $I_{i}$ (instead of in $I_{i+1}$ ).

The proof of the previous proposition also shows the following.

Corollary 8.4. In the setting of the previous proposition, suppose that $\hat{I}$ is a terminating interval with $I_{i-1} \subset \hat{I} \subset I_{i-2}$. Let $\hat{K}$ be an interval $\delta^{\prime}$-well-inside $\hat{I}$ for some $\delta^{\prime}>0$ with $G_{s_{i-1}} \subset \hat{K}$. Let

$$
U_{s_{i-1}}=D_{\theta}(\hat{K}) \cap \mathbb{C}_{G_{s_{i-1}}} \text { and } U_{j}=\operatorname{Comp}_{G_{j}} f^{-\left(s_{i-1}-j\right)}\left(U_{s_{i-1}}\right) \text {. }
$$

Then there exist an interval $K$ and $\delta>0$ such that

$$
U_{r_{i-1}} \subset D_{\mu \theta}(K)
$$

and $(1+2 \delta) K \subset \mathcal{R}\left(I_{i-1}\right)$. Moreover, there are atmost three times $j$ with $r_{i-1} \leq j \leq s_{i-1}$ and $G_{j} \subset I_{i+1}$.

Corollary 8.4 is the result we need to apply after Proposition 8.2 in Fig. 13. In this application the interval $\hat{I}$ will satisfy $I_{i} \subset \hat{I} \subset I_{i-1}$, and the time $s_{i-1}$ will be substituted by the time $s_{i}^{\prime}$ obtained from Proposition 8.2 (rather than the time $s_{i}$ ). In the next section we will show that $s_{i}^{\prime}$ satisfies the assumption needed in Proposition 8.3.

The next proposition completes the results needed to complete the pullbacks described in Figs. 13 and 14. Note that in Fig. 13 we use Proposition 8.5 for $I_{i}$ instead of $I_{i-1}$, so the indexes have to be changed accordingly.

Proposition 8.5. For each $v>0$ there exists $\mu=\mu(\nu) \in(0,1)$ and for each $\theta \in(0, \pi)$ there exists $\varepsilon>0$ such that the following holds. Let $I_{i-1}$ be terminating with $\left|I_{i-1}\right|<\varepsilon$. Assume that $\mathcal{R}\left(I_{i-1}\right)$ is non-terminating and that

$$
\frac{\left|I_{i-2}\right|}{\left|I_{i+1}\right|}<v \text {. }
$$

Assume $n>i$ and consider a disjoint chain $\left\{G_{j}\right\}_{j=0}^{s}$ with $G_{s}=I_{n}, G_{0} \subset I_{n}, G_{0} \cap$ $\omega\left(c_{0}\right) \neq \emptyset$ and $G_{r_{i-1}} \subset \mathcal{R}\left(I_{i-1}\right)$ for some $0<r_{i-1} \leq s$. Let $s_{i}$ be maximal so that $G_{s_{i}} \subset I_{i}$ with $0 \leq s_{i}<r_{i-1}$ and define

$$
U_{r_{i-1}}=D_{\theta}\left(\mathcal{R}\left(I_{i-1}\right)\right) \cap \mathbb{C}_{G_{r_{i-1}}} \text { and } U_{j}=\operatorname{Comp}_{G_{j}} f^{-\left(r_{i-1}-j\right)}\left(U_{r_{i-1}}\right) \text {. }
$$

Then

$$
U_{s_{i}} \subset D_{\mu \theta}\left(I_{i}\right)
$$

Moreover, there are at most two times $j$ with $G_{j} \subset I_{i}$ and $s_{i} \leq j \leq r_{i-1}$. 
Proof. Let $r$ be the period of $I_{i-1}^{\infty}$. By Proposition 3.20, $\mathcal{R}\left(I_{i-1}\right)$ is $\rho$-free. Let $s^{\prime}<r_{i-1}$ be maximal so that $G_{s^{\prime}} \subset \mathcal{R}\left(I_{i-1}\right)$. Let $\hat{I}:=\mathcal{L}_{G_{s^{\prime}}}\left(\mathcal{R}\left(I_{i-1}\right)\right)$. By Lemma 4.6, pulling back once under the return map to $\mathcal{R}\left(I_{i-1}\right)$ we have that

$$
U_{s^{\prime}} \subset D_{\lambda \theta}(\hat{I})
$$

for $\lambda \in(0,1)$ and $G_{s^{\prime}} \subset \hat{I} \subset \mathcal{R}\left(I_{i-1}\right)$. If $\hat{I}=I_{i}$ we are done, so we suppose that this is not the case. As in Corollary 3.21 we define $V_{0}$ to be the component of $f^{-2 r}\left(\mathcal{R}\left(I_{i-1}\right)\right)$ that contains $\alpha$ in its boundary, $W_{0}$ to be the component of $f^{-2 r}\left(\mathcal{R}\left(I_{i-1}\right)\right)$ that contains $\tau(\alpha)$ in its boundary, $V_{1}$ be the component of $f^{-4 r}\left(\mathcal{R}\left(I_{i-1}\right)\right)$ that contains $\alpha$ in its boundary, and $W_{1}$ be the component of $f^{-4 r}\left(\mathcal{R}\left(I_{i-1}\right)\right)$ that contains $\tau(\alpha)$ in its boundary.

Case 1: By Corollary 3.21, if $\hat{I}$ is different from $V_{0}$ and from $W_{0}$, then there exists $\rho^{\prime}>0$ such that $\hat{I}$ is $\rho^{\prime}$-nice and $\rho^{\prime}$-free. Let $s^{\prime \prime}$ be minimal such that $G_{s^{\prime \prime}} \subset \hat{I}$ with $s_{i}<s^{\prime \prime} \leq s^{\prime}$. For each critical point $c$, there is at most one $j_{c}$ such that $G_{j_{c}} \subset \hat{\mathcal{L}}_{c}\left(I_{i}\right)$ with $s^{\prime \prime} \leq j_{c}<s^{\prime}$. Let $j_{c}$ be the maximal such $G_{j_{c}} \subset \hat{\mathcal{L}}_{c}\left(I_{i}\right)$ for $s^{\prime \prime} \leq j_{c}<s^{\prime}$, where the maximum is taken over all $c \in \operatorname{Crit}(f)$. If $j_{c}$ is not defined let $j_{c}=s^{\prime \prime}$. If $j_{c}>s^{\prime \prime}$ let $s_{1}$ be minimal with $G_{s_{1}} \subset \hat{I}$ and $j_{c}<s_{1} \leq s^{\prime}$. Then if $I_{i}$ is non-terminating, $k\left(\hat{I},\left\{G_{j}\right\}_{j=s_{1}}^{s^{\prime}}\right)$ is bounded by a constant $K(v)$ by Proposition 8.1. If $I_{i}$ is terminating, we split the chain into at most $b$ parts, one for each critical point in $\operatorname{Crit}\left(\hat{I},\left\{G_{j}\right\}_{j=s_{1}}^{s^{\prime}}\right)$, on which we can control the loss of angle using Corollary 4.8 and Proposition 7.1 as in Case (ii) of Proposition 8.2. In any case, we can find $\lambda_{1} \in(0,1)$ and an interval $K_{1}$ which is well-inside $\hat{I}$ such that

$$
U_{s^{\prime \prime}} \subset D_{\lambda_{1} \theta}\left(K_{1}\right) \text {. }
$$

By Corollary 4.8 there exists $\mu \in(0,1)$ so that

$$
U_{s_{i}} \subset D_{\mu \theta}\left(I_{i}\right)
$$

Case 2: If $\hat{I}=W_{0}$ let $p$ be maximal such that $G_{p} \subset \mathcal{R}\left(I_{i-1}\right)$ and $s_{i} \leq p<s^{\prime}$. Our goal in this case is to get inside a $\rho$-nice interval, so that we can make use of the argument of the previous case. Applying Lemma 4.6 we can find $\lambda^{\prime}>0$ such that

$$
U_{p} \subset D_{\lambda^{\prime} \theta}(J),
$$

where $J=\mathcal{L}_{G_{p}}\left(W_{0}\right)$. If $p=s_{i}$ we are done with the proof of the proposition, so let us assume this is not the case. If $J$ is not contained in $V_{0}$ or $W_{0}$, then Corollary 3.21 implies that there exists $\rho^{\prime}>0$ such that $J \subset \mathcal{L}_{G_{p}}\left(\mathcal{R}\left(I_{i-1}\right)\right)$ for some $\mathcal{L}_{G_{p}}\left(\mathcal{R}\left(I_{i-1}\right)\right)$ which is $\rho^{\prime}$-nice and $\rho^{\prime}$-free. In this case we can proceed as in Case 1 to get (8.1). If $J \subset V_{0}$, then the fact that $V_{0}$ has size comparable to the size of $\mathcal{R}\left(I_{i-1}\right)$ implies there exists $\rho_{1}>0$ such that $\left(1+2 \rho_{1}\right) J \subset V_{0}$. Applying Theorem $3.2(2)$ we can find $\rho_{1}^{\prime}>0$ so that the following holds. Given $x \in J \cap \omega\left(c_{0}\right),\left(1+2 \rho_{1}^{\prime}\right) \mathcal{L}_{x}(J) \subset \mathcal{L}_{x}\left(V_{0}\right) \subset J$. Which means that $J$ is $\rho_{1}^{\prime}$-nice. Shrinking $\rho_{1}^{\prime}$ if necessary we can assume that $J$ is $\rho_{1}^{\prime}$ free. Now we can proceed as in Case 1 to get (8.1). If $J \subset W_{0}$, then a similar argument implies the existence of $\rho_{1}^{\prime}>0$ so that the following holds. Given $x \in J \cap \omega\left(c_{0}\right)$, then $\left(1+2 \rho_{1}^{\prime}\right) \mathcal{L}_{x}(J) \subset \mathcal{L}_{x}\left(W_{0}\right)=J$. Which means that $J$ is $\rho_{1}^{\prime}$-nice. Again, shrinking $\rho_{1}^{\prime}$ if necessary we can assume that $J$ is $\rho_{1}^{\prime}$-free.

Case 3: Assume $\hat{I}=V_{0}$. If $G_{s^{\prime}}$ is at most the second return of $G_{s_{i}}$ under the first return map to $\mathcal{R}\left(I_{i-1}\right)$, then we can get (8.1) applying Lemma 4.6 at most twice, so 
let us assume this is not the case. In this case we will pull back along the outermost orientation preserving monotone branch to end up in a $\rho$-nice interval or in $W_{0}$ as in the previous cases. We do not make use of Proposition 6.1, since we do not have fundamental domains on both sides of this branch. Instead, we use the free space to give us intervals at the boundary of $V_{0}$ whose pullbacks shrink exponentially. We will use the same notation as in the proof of (3) in Proposition 3.20. Let $\hat{V}_{0}=\operatorname{Comp}_{\alpha} f^{-r}\left(V_{0}\right) \cup V_{0}$, $\hat{V}_{1}=\operatorname{Comp}_{\alpha} f^{-r}\left(V_{1}\right) \cup V_{1}$ and $\hat{V}_{j+1}=\operatorname{Comp}_{\alpha} f^{-r}\left(\hat{V}_{j}\right)$ for $j \in \mathbb{N}$. From the proof of Proposition 3.20 we know that $\left.f^{2 r}\right|_{V_{1}}$ is monotone and that there exists $\delta_{1}>0$ such that $\left(1+2 \delta_{1}\right) \hat{V}_{2} \subset \hat{V}_{1}$. Since $G_{s_{i}} \subset I_{i}$ there exists $G_{j}$ with $s_{i} \leq j<s^{\prime}$ such that $G_{j} \subset \mathcal{L}_{x}\left(\mathcal{R}\left(I_{i-1}\right)\right)$, for some $\mathcal{L}_{x}\left(\mathcal{R}\left(I_{i-1}\right)\right)$ different from $V_{0}$. Let $q^{\prime}$ be minimal such that $s_{i} \leq q^{\prime}<s^{\prime}, G_{q^{\prime}}$ is not contained in $V_{1}$ and for each $q^{\prime}<j<s^{\prime}$ with $G_{j} \subset \mathcal{R}\left(I_{i-1}\right)$ we have that $G_{j} \subset V_{1}$. Set $q=s^{\prime}-2 r$, note that $s^{\prime}-2 r>0$ by assumption. By Lemma 4.6 we can find $\lambda_{1} \in(0,1)$ so that

$$
U_{q} \subset D_{\lambda_{1} \theta}\left(V_{2}\right)
$$

If $q^{\prime}=q$, under one more pull back we are in one of the previous cases so assume $q>q^{\prime}$. Since $\hat{V}_{2}$ is $\rho^{\prime}$ well-inside $\hat{V}_{1}$ and $\mathcal{R}\left(I_{i-1}\right)$ is $\delta$-free, taking $\delta^{\prime \prime}=\min \left\{\rho^{\prime}, \delta\right\}$ we get that $V_{2}$ is $\delta^{\prime \prime}$ well-inside the monotone branch of $f^{r}$ containing $\alpha$. Let $V_{n_{0}+N}$ be as in Case 3 of the proof of Proposition 3.20. Then, $V_{n_{0}+N} \cap \omega\left(c_{0}\right)=\varnothing$ and the size of $V_{n_{0}+N}$ is comparable to the size of $V_{0}$. Let $V$ be the preimage of $V_{n_{0}+N}$ under $\left.f^{2 r}\right|_{V_{1}}$. If there is a turning point $c$ of $\left.f^{2 r}\right|_{V_{0}}$ let $\tilde{V}$ be the preimage of $V_{n_{0}+N}$ under $f^{2 r}$ that is symmetric to $V$ with respect to $c$. If $\left.f^{2 r}\right|_{V_{0}}$ is monotone let $V^{\prime}$ be the preimage of $V_{n_{0}+N}$ under $f^{2 r}$ that contains $\tau(\alpha)$ and let $\tilde{V}$ be the preimage of $V^{\prime}$ under $\left.f^{2 r}\right|_{V_{0}}$. We can apply Lemma 3.10 to $\left.f^{r}\right|_{I_{i-1}^{\infty}}$, so we get real bounds for $\left.f^{2 r}\right|_{V_{0}}$; these bounds along with the definition of $V_{n_{0}+N}$ imply that the sizes of $V$ and $\tilde{V}$ are comparable to the size of $V_{0}$. By construction, the pullbacks of $\tilde{V}$ under $\left.f^{2 r}\right|_{V_{1}}$ are disjoint. The pullbacks of $V$ under $f^{2 r}$ are not disjoint but they will shrink exponentially. To see this, define $F_{q}=V$ and $F_{j}=\left(f^{2 r} \mid V_{1}\right)^{-1}\left(F_{j+1}\right)$ for $q^{\prime} \leq j<q$. By Theorem $3.2(2)$, there exists $\eta \in(0,1)$ universal such that for each $j, j=q^{\prime}, \ldots, q-1,\left|F_{j+1}\right|<\eta\left|F_{j}\right|$. Hence $\sum_{j=q^{\prime}}^{q}\left|F_{j}\right|$ is bounded by a constant depending on $\varepsilon$. We can apply the same proof used in Proposition 6.1 to pull back from time $q$ to time $q^{\prime}$. In this case the fact that the pullbacks of $V$ are not disjoint will not matter since we have exponential contraction of the chain $\left\{F_{j}\right\}_{j=q^{\prime}}^{q}$ and this is enough to control the loss of angle. Thus, we can find $\lambda_{2} \in(0,1)$ so that

$$
U_{q^{\prime}} \subset D_{\lambda_{2} \theta}\left(V_{2}\right)
$$

Let $t_{1}<q^{\prime}$ be maximal such that $G_{t_{1}} \subset \mathcal{R}\left(I_{i-1}\right)$,. If $G_{t_{1}} \cap V_{0}=\emptyset$ we can apply Lemma 4.6, since $V_{0}$ is $\rho^{\prime}$-free, to get to Case 2 or Case 3. If $\hat{G}_{t_{1}} \subset V_{0}$, then after one more pull back we end up either in one of the previous cases or inside a landing domain to $V_{0}$ that is well-inside $V_{0}$, which will be $\delta^{\prime \prime}$-nice, $\delta^{\prime \prime}$-free, for some $\delta^{\prime \prime}>0$, and argue as in Case 1.

Combinatorial Remark 7. If in the previous proposition there exist two intervals $G_{j} \subset$ $I_{i}$, we must have that $G_{s_{i}}$ and $G_{r_{i-1}}$ are such intervals. 


\subsection{The non-renormalizable case.}

Proposition 8.6 (Non-renormalizable case). For each $v>0$ there exists $\mu=\mu(v) \in$ $(0,1)$ and for each $\theta \in(0, \pi)$ there exists $\varepsilon>0$ such that the following holds. Suppose that $I_{i-1}$ and $I_{i}$ are non-terminating, $\left|I_{i-1}\right|<\varepsilon$ and

$$
\frac{\left|I_{i-2}\right|}{\left|I_{i+1}\right|}<v .
$$

Assume $n>i$ and consider a disjoint chain $\left\{G_{j}\right\}_{j=0}^{s}$ with $G_{s}=I_{n}, G_{0} \subset I_{n}, G_{s_{i-1}} \subset$ $I_{i-1}$ for $0<s_{i-1} \leq s$. Let $p_{i-1}$ be so that $I_{i}$ is a pullback of $I_{i-1}$ under $f^{p^{i-1}}$. Suppose $x \in G_{0} \cap \omega\left(c_{0}\right)$ is so that $f^{s_{i-1}}(x) \in I_{i-1}$ for some $p_{i-1}<s_{i-1} \leq s$ and that $f^{s_{i}^{\prime}}(x) \in I_{i}$ for some $0 \leq s_{i}^{\prime}<s_{i-1}-p_{i-1}$. Assume that

$$
\#\left\{k ; s_{i}^{\prime} \leq k \leq s_{i-1} \text { and } f^{k}(x) \in I_{i}\right\} \leq 4 .
$$

Let

$$
U_{s_{i-1}}=D_{\theta}\left(I_{i-1}\right) \cap \mathbb{C}_{G_{s_{i-1}}} \text { and } U_{j}=\operatorname{Comp}_{G_{j}} f^{-\left(s_{i-1}-j\right)}\left(U_{s_{i-1}}\right) .
$$

Then

$$
U_{s_{i}^{\prime}} \subset D_{\mu \theta}\left(I_{i}\right)
$$

where $\mu=\mu(v, \delta)$.

Proof. By Corollary 3.25 there exists $\delta>0$ such that $I_{i-1}$ is $\delta$-free. Since $f^{s_{i}^{\prime}}(x) \in I_{i}$, $f^{p_{i-1}+s_{i}^{\prime}}(x) \in I_{i-1}, f^{s_{i-1}}(x) \in I_{i-1}$ and $s_{i}^{\prime}<s_{i-1}-p_{i-1}$ we can decompose $f^{s_{i-1}}$ in the following way $f^{s_{i-1}}(x)=R_{I_{i-1}}^{q} \circ f^{p_{i-1}} \circ f^{s_{i}^{\prime}}(x)$ with $q \geq 1$. Let $f^{s^{\prime}}=R_{I_{i-1}}^{q-1} \circ$ $f^{p_{i-1}} \circ f^{s_{i}^{\prime}}(x)$. By Proposition 6.2 we know that there exists $\lambda>0$ such that

$$
U_{s^{\prime}} \subset D_{\lambda \theta}\left(\mathcal{L}_{f^{s^{\prime}}(x)}\left(I_{i-1}\right)\right)
$$

with $G_{s^{\prime}} \subset \mathcal{L}_{f^{s^{\prime}(x)}}\left(I_{i-1}\right) \subset(1+2 \delta) \mathcal{L}_{f^{s^{\prime}}(x)}\left(I_{i-1}\right) \subset I_{i-1}$. Let $s^{\prime \prime}$ be maximal with $s_{i}^{\prime}+p_{i-1} \leq s^{\prime \prime}+p_{i-1} \leq s^{\prime}$ and $G_{s^{\prime \prime}} \subset I_{i}$. If $s^{\prime}=s^{\prime \prime}+p_{i-1}$, do nothing. If $s^{\prime \prime}+p_{i-1}<s^{\prime}$ let $s^{\prime \prime}+p_{i-1}=k_{0}<k_{1}<\cdots<k_{m}=s^{\prime}$ be such that $G_{k_{j}} \in I_{i-1}$. By maximality of $s^{\prime \prime}$ and Lemma 2.3 there are at most three intervals $G_{k_{j}}$ such that $G_{k_{j}} \subset I_{i}$. Let $k_{j_{0}}$ be maximal such that $j_{0} \in\{0, \ldots m\}$ and $G_{k_{0}} \in I_{i}$. Proposition 7.1 allows to control the loss of angle when we pull back from time $s^{\prime}$ to time $k_{j_{0}+1}$ and Corollary 4.8 controls the loss of angle pulling back from time $k_{j_{0}+1}$ to $k_{j_{0}}$. Repeating this procedure at most three times, we have that

$$
U_{s^{\prime \prime}+p_{i-1}} \subset D_{\lambda^{\prime} \mu \theta}\left(K^{\prime}\right)
$$

where $K^{\prime}$ is well-inside $I_{i-1}$ and $\mu$ depends only on $\hat{k}\left(I_{i-1}, I_{i}\right)$, which by Lemma 8.1 is bounded by a constant that depends on $v$. If $I_{i-2}$ is non-terminating, then $I_{i-1}$ is $\delta$ externally free, $\delta$-internally free, and $I_{i}$ is a pullback of $I_{i-1}$ of bounded order (depending only on $b$ ), so, since $\left|I_{i-1}\right| /\left|I_{i}\right|<v$, we can apply Proposition 6.2 and find $\mu^{\prime}>0$ such that

$$
U_{s^{\prime \prime}} \subset D_{\mu^{\prime} \theta}\left(I_{i}\right)
$$


If $I_{i-2}$ is terminating, then as in the Case 3 of the proof of the previous proposition, there are puzzle pieces $U$ and $V$ attached to $\alpha$ and $\tau(\alpha)$ in $\mathcal{R}\left(I_{i-2}\right)$, that are disjoint from $\omega\left(c_{0}\right)$ and with size comparable to $\left|\mathcal{R}\left(I_{i-2}\right)\right|$, pulling these back to $I_{i-1}$ we obtain puzzle pieces $U_{1}, V_{1}$ attached to $\partial I_{i-1}$, with size comparable to $\left|I_{i-1}\right|$ that are disjoint from $\omega\left(c_{0}\right)$. So arguing as in Proposition 6.2, we get that

$$
U_{s^{\prime \prime}} \subset D_{\mu^{\prime} \theta}\left(I_{i}\right) \text {. }
$$

If $s^{\prime \prime}=s_{i}^{\prime}$ we are done. In case $s^{\prime \prime}>s_{i}^{\prime}$. Since $I_{i-1}$ is $\delta$-free Corollary 3.5 implies that $I_{i}$ is $\delta^{\prime}$-free, for some $\delta^{\prime}>0$. This and the fact that $\#\left\{k ; s_{i}^{\prime} \leq k \leq s_{i-1}\right.$ and $\left.f^{k}(x) \in I_{i}\right\}<4$ allows us to prove the proposition applying Lemma 4.6 at most twice.

8.3. Large scaling factors. In the remainder of this section we show that as soon as one has a large scaling factor, the corresponding first return map extends to an analytic box mapping or a qr box mapping, see Definitions 1.1 and 1.2. The results are stated for maps in $\mathcal{A}_{\underline{b}}^{3}$, but if the maps are in $\mathcal{A}_{\underline{b}}$ the qr box mappings are box mappings.

Proposition 8.7. There exist $\varepsilon>0$ and $v_{1} \geq 1$ such that the following holds. Suppose that $I_{i-2}$ and $I_{i-1}$ are non-terminating and $\left|I_{i-2}\right|<\varepsilon$. If

$$
\frac{\left|I_{i-2}\right|}{\left|I_{i-1}\right|}>v_{1},
$$

then

(1) the first return map to $\Gamma\left(I_{i-1}^{1}\right)$ restricted to components that intersect $\omega\left(c_{0}\right)$ extends to a qr quasi-box mapping with range $D_{\pi / 2}\left(\Gamma\left(I_{i-1}^{1}\right)\right)$;

(2) if $I_{i}$ is non-terminating, then the first return map to $I_{i}$ restricted to the components of the domain intersecting $\omega\left(c_{0}\right)$ extends to a qr box mapping with range $D_{\pi / 2}\left(I_{i}\right)$.

Proof. Let us prove the first statement. To simplify the notation let $J=\Gamma\left(I_{i-1}^{1}\right)$. By Theorem 3.1 (a) there exists $\rho>0$ so that $I_{i-1}$ is $\rho$-externally free. Moreover, if $c_{0}$ is even we know that $I_{i-1} \backslash(1+2 \rho)^{-1} I_{i-1}$ is disjoint from $\omega\left(c_{0}\right)$. Since $J$ is a pullback of $I_{i-1}$ of bounded order there exists $\rho^{\prime}>0$ so that $J$ is $\rho^{\prime}$-externally free; if $c_{0}$ is even we additionally have that $J \backslash\left(1+2 \rho^{\prime}\right)^{-1} J$ is disjoint from $\omega\left(c_{0}\right)$. Fix $C>0$ from Lemma 3.24, then $J$ is $C$-nice. Pick $x \in J \cap \omega\left(c_{0}\right)$ and let $s$ be the return time of $x$ to $J$. Since $J$ is $\rho^{\prime}$-externally free the return time of $x$ to $\left(1+2 \rho^{\prime}\right) J$ is $s$. Let us first consider the case when $c_{0}$ is even. Let $V=D_{\pi / 2}(J)$ and $U=\operatorname{Comp}_{x} f^{-s}(V)$. Since, in this case, $J \backslash\left(1+2 \rho^{\prime}\right)^{-1} J$ is disjoint from $\omega\left(c_{0}\right)$ we can apply Lemma 4.6 to find $\lambda \in(0,1)$, depending only on $\rho$, so that $U \subset D_{\lambda \pi / 2}\left(\mathcal{L}_{x}(J)\right)$. Taking $C$ be big enough we get that

$$
D_{\lambda \pi / 2}\left(\mathcal{L}_{x}(J)\right) \subset D_{\pi / 2}\left((1+2 C) \mathcal{L}_{x}(J)\right) \subset D_{\pi / 2}(J) .
$$

In this case the first part of the proposition follows taking $v>1 / \varepsilon$, where $\varepsilon$ is the constant associated to $C$ in Lemma 3.24. Let us now assume that $c_{0}$ is odd; then all the critical points in $\omega\left(c_{0}\right)$ are odd. Since $J$ is $\rho^{\prime}$-externally free, $\tilde{I}:=\left(1+\rho^{\prime}\right) J$ is $\rho^{\prime \prime}$-free for some $\rho^{\prime \prime}>0$ that depends only on $\rho$. Note that since $J$ is $\rho^{\prime}$-externally free $\mathcal{L}_{x}(\tilde{I}) \subset J$. Let $V=D_{\pi / 2}(\tilde{I})$ and $U=\operatorname{Comp}_{x} f^{-s}(V)$. Since $\tilde{I}$ is $\rho^{\prime \prime}$-free we can apply Lemma 4.6 to find $\lambda^{\prime} \in(0,1)$, depending on $\rho$, so that $U \subset D_{\lambda^{\prime} \pi / 2}\left(\mathcal{L}_{x}(\tilde{I})\right)$. Since all the critical points in $\omega\left(c_{0}\right)$ are odd $\left.f^{s}\right|_{\mathcal{L}_{x}(J)}$ and $\left.f^{s}\right|_{\mathcal{L}_{x}(\hat{I})}$ (the return maps to $J$ and $\tilde{I}$ ) are monotone and 
there exists $\kappa>0$, depending on $\rho$, the number of critical points of $f$ and their orders, so that $\left|\mathcal{L}_{x}(J)\right|>\kappa\left|\mathcal{L}_{x}(\tilde{I})\right|$. This means that there exists $C^{\prime}>0$ depending on $\kappa$ and $C$, with $C^{\prime} \rightarrow \infty$ as $C \rightarrow \infty$, so that

$$
\left(1+2 C^{\prime}\right) \mathcal{L}_{x}(\tilde{I}) \subset J
$$

since $(1+2 C) \mathcal{L}_{x}(J) \subset J$. Taking $C$ big enough we get

$$
D_{\lambda^{\prime} \pi / 2}\left(\mathcal{L}_{x}(\tilde{I})\right) \subset D_{\pi / 2}\left(\left(1+2 C^{\prime}\right) \mathcal{L}_{x}(\tilde{I})\right) \subset D_{\pi / 2}(J) .
$$

In this case the first part of the proposition follows taking $v>1 / \varepsilon$, where $\varepsilon$ is the constant associated to $C$ in Lemma 3.24.

Let us now prove the second statement. By Theorem 3.1 (a), $I_{i}$ is $\rho$-externally free. Moreover, if $c_{0}$ is even we know that $I_{i} \backslash(1+2 \rho)^{-1} I_{i}$ is disjoint from $\omega\left(c_{0}\right)$. Fix $x \in$ $\omega\left(c_{0}\right) \cap I_{i}$. We know the return time of $x$ to $I_{i}$ is the same as its return time to $(1+2 \rho) I_{i}$; let this common return time be equal to $q$. Let $\tilde{F}_{n}$ be defined as in the proof of Lemma 3.24. From Lemma 3.24 we know that there exists $C>0$ so that $I_{i}$ is $C-$ strongly nice and that

$$
\mathcal{L}_{x}\left(I_{i}\right) \subset(1+2 C) \mathcal{L}_{x}\left(I_{i}\right) \subset \mathcal{L}_{x}\left(\Gamma^{T} \mathcal{B}\left(\tilde{F}_{n}\right)\right) \subset I_{i} .
$$

Note that $(1+C) \mathcal{L}_{x}\left(I_{i}\right)$ is compactly contained in $\mathcal{L}_{x}\left(\Gamma^{T} \mathcal{B}\left(\tilde{F}_{n}\right)\right)$. Recall that given $x, y \in I_{i}$ so that $\mathcal{L}_{x}\left(I_{i}\right)$ is different from $\mathcal{L}_{y}\left(I_{i}\right)$, then $\mathcal{L}_{x}\left(\Gamma^{T} \mathcal{B}\left(\tilde{F}_{n}\right)\right)$ is disjoint from $\mathcal{L}_{y}\left(\Gamma^{T} \mathcal{B}\left(\tilde{F}_{n}\right)\right)$. Assume $c_{0}$ is even and let $V=D_{\pi / 2}\left(I_{i}\right)$ and $U=\operatorname{Comp}_{x} f^{-q}(V)$. Since, in this case, $I_{i} \backslash(1+2 \rho)^{-1} I_{i}$ is disjoint from $\omega\left(c_{0}\right)$ we can apply Lemma 4.6 to find $\lambda_{1} \in(0,1)$ so that $U \subset D_{\lambda_{1} \pi / 2}\left(\mathcal{L}_{x}\left(I_{i}\right)\right)$. Taking $C$ sufficiently large we get that

$$
D_{\lambda_{1} \pi / 2}\left(\mathcal{L}_{x}\left(I_{i}\right)\right) \subset D_{\pi / 2}\left((1+C) \mathcal{L}_{x}\left(I_{i}\right)\right),
$$

so the proposition follows in this case. If $c_{0}$ is odd we let $\tilde{I}=(1+\rho) I_{i}$. By definition, $\tilde{I}$ is $\rho_{1}$-free for some $\rho_{1}>0$. Let $V=D_{\pi / 2}(\tilde{I})$ and $U=\operatorname{Comp}_{x} f^{-q}(V)$. By Lemma 4.6 there exists a constant $\lambda_{2} \in(0,1)$ so that $U \subset D_{\lambda_{2} \pi / 2}\left(\mathcal{L}_{x}(\tilde{I})\right)$. We know that $\left|\mathcal{L}_{x}\left(I_{i}\right)\right|>$ $\kappa\left|\mathcal{L}_{x}(\tilde{I})\right|$. This means that there exists $C^{\prime}>0$ depending on $\kappa$ and $C$, with $C^{\prime} \rightarrow \infty$ as $C \rightarrow \infty$, so that

$$
\left(1+2 C^{\prime}\right) \mathcal{L}_{x}(\tilde{I}) \subset \mathcal{L}_{x}\left(\Gamma^{T} \mathcal{B}\left(\tilde{F}_{n}\right)\right)
$$

Making $C$ big enough we get

$$
D_{\lambda_{2} \pi / 2}\left(\mathcal{L}_{x}(\tilde{I})\right) \subset D_{\pi / 2}\left(\left(1+C^{\prime}\right) \mathcal{L}_{x}(\tilde{I})\right) .
$$

Since $\left(1+C^{\prime}\right) \mathcal{L}_{x}(\tilde{I})$ is compactly contained in $\mathcal{L}_{x}\left(\Gamma^{T} \mathcal{B}\left(\tilde{F}_{n}\right)\right)$ the proposition follows.

Corollary 8.8. There exist $\varepsilon>0$ and $v_{1} \geq 1$ such that the following holds. Suppose that $I_{i-2}$ and $I_{i-1}$ are non-terminating, $\left|I_{i-2}\right|<\varepsilon$, and

$$
\frac{\left|I_{i-2}\right|}{\left|I_{i-1}\right|}>v_{1} \text {. }
$$

Let $\hat{I}_{i}=\cup \hat{\mathcal{L}}_{c}\left(I_{i}\right)$, where the union is taken over all $c \in \operatorname{Crit}(f) \cap \omega\left(c_{0}\right)$. Then, the first return map to $\hat{I}_{i}$ restricted to the domains intersecting $\omega\left(c_{0}\right)$ extends to a qr box mapping $F: \mathcal{U} \rightarrow \mathcal{V}$, where $\left.F\right|_{U}$ is at most unicritical for all $U \in \mathcal{U}$. Furthermore, there exists a universal constant $\rho>0$ so that each component in $\mathcal{U}$ has $\rho$-bounded geometry. 
Proof. Let $v_{1} \geq 1$ from Proposition 8.7. Observe that since $I_{i}$ is $\rho$-externally free, taking $v_{1}$ larger if necessary, we can guarantee the following. For each $c \in \operatorname{Crit}(f) \cap \omega\left(c_{0}\right), c \neq$ $c_{0}$, let $r_{c}>0$ be minimal so that $f^{r_{c}}(c) \in I_{i}, V_{c_{0}}=D_{\pi / 2}\left(I_{i}\right)$ and $V_{c}=\operatorname{Comp}_{c} f^{-r_{c}}\left(V_{0}\right)$. Then for any two distinct $c, c^{\prime} \in \operatorname{Crit}(f) \cap \omega\left(c_{0}\right)$, we have that $V_{c} \cap V_{c^{\prime}}=\emptyset$. Thus the qr box mapping given by Proposition 8.7 induces a qr box mapping $F: \mathcal{U} \rightarrow \mathcal{V}$ where $\mathcal{V}$ is a neighbourhood of $\operatorname{Crit}(f) \cap \omega\left(c_{0}\right)$, where the map $\left.F\right|_{U}$ has at most one critical point for each $U \in \mathcal{U}$. Recall that there exists a constant $\delta>0$ such that either $I_{i}$ is $\delta$-free or all the critical points in $\omega\left(c_{0}\right)$ are odd. By Corollary 5.7 and Lemma 4.3, there exists a constant $\theta^{\prime}=\theta^{\prime}(\delta) \in(\pi / 2, \pi)$ such that if $I_{i}$ is $\delta$-free, then each component $U$ of $\mathcal{U}$ contains $D_{\theta^{\prime}}(U \cap \mathbb{R})$. Whether $I_{i}$ is $\delta$-free or not, since $F$ is a quasiregular map with bounded degree and bounded qc distortion, there exists $\delta^{\prime}>0$ such that each component of $U$ has $\delta^{\prime}$-bounded geometry.

Lemma 8.9. There exist constants $v_{1} \geq 1$ and $\varepsilon>0$ such that the following holds. Suppose that $I_{i-1}$ is terminating, $\left|I_{i-1}\right|<\varepsilon$ and

$$
\left|I_{i-1}\right| /\left|I_{i-1}^{\infty}\right|>v_{1} .
$$

Then the first return map to $I_{i-1}^{\infty}$ extends to a qr box mapping with range $D_{\pi / 2}\left(I_{i-1}^{2}\right)$.

Proof. Let $r$ be the return time of $c_{0}$ to $I_{i-1}$. By Lemma 3.10 we know that given $\delta_{1}>0$ there exists $\kappa>0$ so that if $\left|I_{i-1}^{2}\right|>\delta_{1}\left|I_{i-1}\right|$, then $\left|I_{i-1}^{\infty}\right|>\kappa\left|I_{i-1}\right|$. This means that taking $v_{1}$ small enough there exists $C^{\prime}>0, C^{\prime} \rightarrow \infty$ as $v_{1} \rightarrow \infty$ so that $\left(1+2 C^{\prime}\right) I_{i-1}^{2} \subset I_{i-1}$. Since $I_{i-1}$ is terminating, $c_{0}$ is an even critical point, either $I_{i-1}^{1}$ is deep-inside $I_{i-1}$ or $I_{i-1}^{2}$ is deep-inside $I_{i-1}^{1}$. In any case we get that $I_{i-1}^{2}$ is deep-inside $I_{i-1}^{1}$ and $I_{i-1}^{3}$ is deep-inside $I_{i-1}^{2}$. In particular $I_{i-1}^{2}$ is $\delta$-free for some $\delta>0$. Note that we can fix $\delta$ so that $I_{i-1}^{2}$ is $\delta$-free for all $\nu_{1}>C_{0}$ for some $C_{0}>0$. Let $V=D_{\pi / 2}\left(I_{i-1}^{2}\right)$ and $U=\operatorname{Comp}_{c_{0}} f^{-r}(V)$. By Lemma 4.6 there exists $\lambda \in(0,1)$, depending on $\delta$, so that

$$
U \subset D_{\lambda \pi / 2}\left(I_{i-1}^{3}\right)
$$

Making $v_{1}$ big enough we can guarantee that $\left(1+2 C^{\prime \prime}\right) I_{i-1}^{3} \subset I_{i-1}^{2}$ for some $C^{\prime \prime}=$ $C^{\prime \prime}\left(v_{1}\right)>0$ so that

$$
D_{\lambda \pi / 2}\left(I_{i-1}^{3}\right) \subset D_{\pi / 2}\left(\left(1+C^{\prime \prime}\right)\left(I_{i-1}^{3}\right)\right)
$$

note that $\left(1+C^{\prime \prime}\right)\left(I_{i-1}^{3}\right)$ is compactly contained in $I_{i-1}^{2}$, so the proposition follows.

A direct consequence of the previous lemma we have:

Corollary 8.10. There exist constants $v_{1} \geq 1$ and $\varepsilon>0$ such that the following holds. Suppose that $I_{i-1}$ is non-terminating, $I_{i}$ is terminating, $\left|I_{i-1}\right|<\varepsilon$ and

$$
\left|I_{i-1}\right| /\left|I_{i}\right|>v_{1} \text {. }
$$

Then the first return map to $I_{i}^{\infty}$ extends to a qr box mapping with range $D_{\pi / 2}\left(I_{i}^{2}\right)$.

Proof. Since $I_{i}$ is terminating $c_{0}$ is even and $I_{i}$ and $I_{i} \backslash(1+\delta)^{-1} I_{i} \cap \omega\left(c_{0}\right)$ is empty. Since $I_{i-1}$ is non-terminating we can apply Corollary 3.4 to conclude $I_{i}^{1}$ is deep-inside $I_{i}$. Hence there exists $C>0$ large so that $(1+2 C) I_{i}^{1} \backslash I_{i}$ is disjoint from $\omega\left(c_{0}\right)$, and the proof follows as in the previous lemma. 
Combining the results from this section we obtain the following:

Proposition 8.11. There exist constants $v_{1} \geq 1$ and $\varepsilon>0$ such that the following holds. Suppose that $I_{i-1}$ is terminating, $\left|I_{i-1}\right|<\varepsilon$ and

$$
\left|I_{i-1}\right| /\left|I_{i}\right|>v_{1}
$$

Then we have one of the following:

(1) the first return map to $I_{i-1}^{\infty}$ extends to a qr box mapping with range $D_{\pi / 2}\left(I_{i-1}^{2}\right)$;

(2) $I_{i}$ is terminating and the first return map to $I_{i}^{\infty}$ extends to a qr box mapping with range $D_{\pi / 2}\left(I_{i}^{2}\right)$

(3) $I_{i}$ and $I_{i+1}$ are non-terminating and the first return map to $I_{i+1}$ restricted to the components of the domain intersecting $\omega\left(c_{0}\right)$ extends to a qr box mapping with range $D_{\pi / 2}\left(I_{i+1}\right)$;

(4) $I_{i}$ is non-terminating, $I_{i+1}$ is terminating and the first return map to $I_{i+1}^{\infty}$ extends to a qr box mapping with range $D_{\pi / 2}\left(I_{i+1}^{2}\right)$.

Proof. Note that since $I_{i-1}$ is terminating, $c_{0}$ is even. If $I_{i-1}^{\infty}$ is small compared to $I_{i-1}$, by Lemma $8.9,(1)$ holds. So assume the size of $I_{i-1}^{\infty}$ is comparable to the size of $I_{i-1}$. By Lemma 3.10, the size of $\mathcal{R}\left(I_{i-1}\right)$ is comparable to the size of $I_{i-1}$. In particular, $\mathcal{R}\left(I_{i-1}\right)$ is not periodic, for otherwise we would have that $\left|I_{i}\right|$ is comparable to $\left|I_{i-1}\right|$. Since $I_{i}$ is deep-inside $\mathcal{R}\left(I_{i-1}\right)$, Corollary 3.4 implies that $I_{i}$ is $C$-nice for some $C>0$, with $C \rightarrow \infty$ as $\nu_{1} \rightarrow \infty$. If $I_{i}$ is terminating, we can take $C$ so big that $\left(1+2 C^{\prime}\right) I_{i}^{\infty} \subset I_{i}$ with $C^{\prime} \rightarrow \infty$ as $C \rightarrow \infty$, and (2) follows from Lemma 8.9. If $I_{i}$ is non-terminating, by Theorem $3.1(\mathrm{~d}), I_{i+1}$ is $C^{\prime \prime}$-nice and $C^{\prime \prime}$-externally free, with $C^{\prime \prime} \rightarrow \infty$ as $C \rightarrow \infty$. If $I_{i+1}$ is terminating, (4) follows from Corollary 8.10. If $I_{i+1}$ is non-terminating we argue as follows. Let $x \in \omega\left(c_{0}\right) \cap I_{i+1}$, with return time to $I_{i+1}$ equal to $r$. Let $V=D_{\pi / 2}\left(I_{i+1}\right)$ and $U=\operatorname{Comp}_{x} f^{-r}(V)$. By 4.6 we know there exists $\lambda \in(0,1)$ so that $U \subset D_{\lambda \pi / 2}\left(\mathcal{L}_{x}\left(I_{I_{i+1}}\right)\right)$. Making $C^{\prime \prime}$ sufficiently large we can guarantee

$$
D_{\lambda \pi / 2}\left(\mathcal{L}_{x}\left(I_{i+1}\right)\right) \subset D_{\pi / 2}\left(\left(1+C^{\prime \prime}\right) \mathcal{L}_{x}\left(I_{i+1}\right)\right) \subset V .
$$

Since the intervals $\left(1+C^{\prime \prime}\right) \mathcal{L}_{x}\left(I_{i+1}\right)$ are pairwise disjoint (3) holds.

The following result will be used in Sect. 10 to prove complex bounds for renormalizable maps.

\section{Extension to a QR Quasi-Box Mapping}

The results in this section apply to maps $f \in \mathcal{A}_{\underline{b}}$ and to asymptotic holomorphic extensions of maps $f \in \mathcal{A}_{b}^{3}$, as in Sect. 5.2. We recall that we assume that the Standing Assumptions on p. 26 hold.

In this section, we will prove that we can associate to $f$ a qr quasi-box mapping (see Theorem 9.3). In particular, if $f$ is a real analytic map the qr quasi-box mapping is quasi-box mapping (Definition 1.2) 
9.1. Some inductive statements used in the construction of a quasi-box mapping. Let $\left\{I_{i}\right\}_{i \geq 0}$ be the generalized enhanced nest, defined in Sect. 2. In the following, we will consider points $x \in I_{n} \cap \omega\left(c_{0}\right)$ and their corresponding first return domains $\mathcal{L}_{x}\left(I_{n}\right)$. Our aim is to show that the first return map to $I_{n}$ restricted to the union of these components can be extended to a qr quasi-box mapping with range $D_{\theta}\left(I_{n-M}\right) \cap \mathbb{C}_{I_{n}}$, for some $M>0$ universal.

Recall that, if the interval $I_{n-j-1}$ is non-terminating there exists $p_{n-j-1}>0$ so that $I_{n-j}$ is a pullback of $I_{n-j-1}$ by $f^{p_{n-j-1}}$.

Proposition 9.1. For every $v \geq 1$ there exists $\mu=\mu(\nu)$ and for each $\theta \in(0, \pi)$ there exists $\varepsilon>0$ such that the following holds. Assume $n>5$ and $\left|I_{n}\right|<\varepsilon$. Let $J=\mathcal{L}_{x}\left(I_{n}\right)$ for a point $x \in I_{n} \cap \omega\left(c_{0}\right)$, and let $s>0$ be its first return time to $I_{n}$. Let $\left\{G_{j}\right\}_{j=0}^{s}$ be the chain with $G_{s}=I_{n}$ and $G_{0}=J$. Fix $M \in \mathbb{N}, 4<M<n$. Assume that for $j=0,1, \ldots, M$

$$
\frac{\left|I_{n-j-1}\right|}{\left|I_{n-j}\right|}<v .
$$

Then there exist integers

$$
s_{n-4}<\cdots<s_{n-M-1}<s_{n-M}:=s
$$

so that the following holds:

(1) $G_{S_{n-i}} \subset I_{n-i}$;

(2) if $I_{n-i}$ and $I_{n-i+1}$ are periodic, $G_{s_{n-i}} \subset I_{n-i+1}$;

(3) if $I_{n-i}$ is non-periodic, there exist atmost six intervals $G_{j} \subset I_{n-i}$ with $s_{n-i} \leq j \leq s$;

(4) if $I_{n-i}$ is periodic, there exist at most five intervals $G_{j} \subset I_{n-i+1}$ with $s_{n-i+1} \leq j \leq s$.

For each $i \in\{4, \ldots, M-1\}$ we let

$$
U_{s_{n-i-1}}=D_{\theta}\left(I_{n-i-1}\right) \cap \mathbb{C}_{G_{s_{n-i-1}}} \text { and } U_{j}=\operatorname{Comp}_{G_{j}} f^{-\left(s_{n-i-1}-j\right)}\left(U_{s_{n-i-1}}\right) \text {, }
$$

for $0 \leq j \leq s_{n-i-1}$, then

(i) if $I_{n-i-1}$ and $I_{n-i}$ are non-terminating,

$$
U_{s_{n-i}} \subset D_{\mu \theta}\left(\mathcal{L}_{G_{s_{n-i}}}\left(I_{n-i}\right)\right)
$$

(ii) if $I_{n-i-1}$ is non-terminating, $I_{n-i}$ is terminating and $i>5$, then there exists an interval $K$ such that

$$
U_{s_{n-i+1}} \subset D_{\mu \theta}(K),
$$

where $G_{s_{n-i+1}} \subset K$ and $K$ is well-inside $I_{n-i+1}$;

(iii) if $I_{n-i-1}$ is terminating, then there exists an interval $K$ such that

$$
U_{S_{n-i}} \subset D_{\mu \theta}(K),
$$

where $G_{s_{n-i}} \subset K$, and $K$ is well-inside $I_{n-i}$. 
Proof. The proof of this proposition will be divided into steps. First we will construct the sequence of times $s_{n-i}$ and then we will do the pullbacks.

Step 1: definition of $s_{n-i}$. We state how we will define the time $s_{n-i}$ if $s_{n-i-1}$ is defined.

Case (a). Assume $I_{n-i-1}$ and $I_{n-i}$ are both non-terminating and there are at least four intervals $G_{j} \subset I_{n-i}$ with $0 \leq j \leq s_{n-i-1}$. By Lemma 2.3 (1), we can choose $s_{n-i}^{\prime}<s_{n-i-1}$ maximal with $G_{s_{n-i}} \subset I_{n-i}$ and $s_{n-i-1}-s_{n-i}^{\prime}>p_{n-i-1}$. By maximality of $s_{n-i}^{\prime}$ and Lemma 2.3 there are at most three times $j, s_{n-i}^{\prime} \leq j \leq s_{n-i-1}$ such that $G_{j} \subset I_{n-i}$ and none of the intervals $G_{j}$ with $s_{n-i} \leq j \leq s_{n-i-1}$ are contained in $I_{n-i+1}$. Since there are at least four intervals $G_{j} \subset I_{n-i}$ with $0 \leq j \leq s_{n-i-1}$ we can define $s_{n-i}$ to be maximal with $s_{n-i}<s_{n-i}^{\prime}$ and $G_{s_{n-i}} \subset I_{n-i}$. Observe that there are at most four intervals $G_{j} \subset I_{n-i}$ with $s_{n-i} \leq j \leq s_{n-i-1}$ and one of them is $G_{s_{n-i}}$.

Case (b). Assume $I_{n-i-1}$ is non-terminating, $I_{n-i}$ is terminating and there are at least three intervals $G_{j} \subset I_{n-i}$ with $0 \leq j \leq s_{n-i-1}$. Let $r$ be the return time of $c_{0}$ to $I_{n-i}$. Since there are at least three intervals $G_{j} \subset I_{n-i}$ with $0 \leq j \leq s_{n-i-1}, s_{i-1}>2 r$ and we can define $s_{n-i}=s_{n-i}^{\prime}$, where $s_{n-i}^{\prime}$ is as in Proposition 8.2. Observe that with this definition there are at most two intervals $G_{j} \subset I_{n-i}$ with $s_{n-i} \leq j \leq s_{n-i-1}$, and one of them is $G_{S_{n-i}}$.

Case (c). Assume $I_{n-i-1}$ is terminating, $I_{n-i} \neq \mathcal{R}\left(I_{n-i-1}\right)$ and there are at least four intervals $G_{j} \subset I_{n-i}$ with $0 \leq j \leq s_{n-i-1}$. From Combinatorial Remark 6 we can define $r_{n-i-1}<s_{n-i-1}$ as in Proposition 8.3 so that there are at most three intervals $G_{j} \subset I_{n-i}$ with $r_{n-i-1} \leq j \leq s_{n-i-1}$. Thus we can define $s_{n-i}<r_{n-i}$ to be maximal such that $G_{s_{n-i}} \subset I_{n-i}$. Even more, if there are four intervals $G_{j} \subset I_{n-i}$ with $s_{n-i} \leq j \leq s_{n-i-1}$ we must have that $G_{S_{n-i-1}}, G_{r_{n-i-1}}$ and $G_{S_{n-i}}$ are in $I_{n-i}$.

Case $(d)$. Assume $I_{n-i-1}$ is terminating, $I_{n-i}=\mathcal{R}\left(I_{n-i-1}\right)$ and there are at least three intervals $G_{j} \subset I_{n-i+1}$ with $0 \leq j \leq s_{n-i-1}$. In this case, we define $r_{n-i-1}<s_{n-i-1}$ as in Proposition 8.3. If $I_{n-i+1} \neq \mathcal{R}\left(I_{n-i}\right)$ or $I_{n-i+1}=\mathcal{R}\left(I_{n-i}\right)$ and $G_{r_{n-i-1}} \subset I_{n-i+1}$ let $s_{n-i}=r_{n-i-1}$. Observe that with this definition there are at most three intervals $G_{j} \subset I_{n-i+1}$ with $s_{n-i} \leq j \leq s_{n-i-1}$. If $I_{n-i+1}=\mathcal{R}\left(I_{n-i}\right)$ and $G_{r_{n-i-1}} \nsubseteq I_{n-i+1}$, then by Proposition 8.3 there are at most two intervals $G_{j} \subset I_{n-i+1}$ with $s_{n-i} \leq j \leq s_{n-i-1}$. If we let $q$ be the return time of $c_{0}$ to $I_{n-i}$ we must have that $G_{r_{n-i-1}-q} \subset I_{n-i+1}$. So in this case we define $s_{n-i}=r_{n-i-1}-q$. Observe that with this definition there are at most three intervals $G_{j} \subset I_{n-i+1}$ with $s_{n-i} \leq j \leq s_{n-i-1}$. Even more, if there exist three intervals $G_{j} \subset I_{n-i+1}$ with $s_{n-i} \leq j \leq s_{n-i-1}$ we must have that $G_{s_{n-i-1}}$ and $G_{S_{n-i}}$ are in $I_{n-i+1}$.

Step 2: construction of the sequence $s_{n-4}<\cdots<s_{n-M}$. We will construct the sequence of times $s_{n-i}$ inductively. To do so we need to check that once $s_{n-i-1}$ has been defined we still have enough visits to $I_{n-i}$ or $I_{n-i+1}$, depending on the case, to define $s_{n-i}$ as in Step 1. Note that if $i+1>4$ there exist at least 16 intervals $G_{j} \subset I_{n-i}$ and that there are at least 8 intervals $G_{j} \subset I_{n-i+1}$. Let us prove the basis of the induction. Since $M>4$ there exist at least 16 intervals $G_{j} \subset I_{n-M+1}$ and at least 8 of them are contained in $I_{n-M+2}$. So we can define $s_{n-M+1}$ using the corresponding case from Step 1. Observe that if $I_{n-M+1}$ is different from $\mathcal{R}\left(I_{n-M}\right)$ there exist at most four intervals $G_{j} \subset I_{n-M+1}$ with $s_{n-M+1} \leq j \leq s$. This shows the induction step in this case. If $I_{n-M+1} \neq \mathcal{R}\left(I_{n-M}\right)$, then by Step 1 we know that there exist at most three intervals $G_{j} \subset I_{n-M+2}$ with $s_{n-M+1} \leq j$. Since at least 8 intervals $G_{j} \subset I_{n-M+2}$ we can define $s_{n-M+2}<s_{n-M+1}$ maximal with $G_{s_{n-M+2}} \subset I_{n-M+2}$. In this case there exist at most 
four intervals $G_{j} \subset I_{n-M+2}$ with $s_{n-M+2} \leq j \leq s$ and the induction step follows. The statement of the proposition says the following: if $I_{n-i}$ is non-periodic, there exist at most six intervals $G_{j} \subset I_{n-+1 i}$ with $s_{n-i+1} \leq j \leq s$; if $I_{n-i}$ is periodic, there exist at most six intervals $G_{j} \subset I_{n-i+1}$ with $s_{n-i} \leq j \leq s$. It is easy to see that the bound is in fact six, and not four like the basis of the induction suggests, by constructing $s_{n-M+2}$ and $s_{n-M+3}$; we will leave this to the reader.

Assume $s_{n-i-1}<s_{n-i-2}<\cdots<s_{n-M}$ are defined, and that they definition has been made according the corresponding case in Step.1 Now we construct the time $s_{n-i}$, for $i>M-1$. Note that if we want to define $s_{n-i}$ we must have that $i+1>4$.

Case $\left(a^{\prime}\right)$ : assume $I_{n-i-1}$ and $I_{n-i}$ are non-terminating. Since $I_{n-i-1}$ is non-terminating the induction hypothesis tells us that there exist at most six intervals $G_{j} \subset I_{n-i-1}$ with $s_{n-i-1} \leq j \leq s$; one of them is $G_{s}$. Thus, there exist at most three intervals $G_{j} \subset I_{n-i}$ with $s_{n-i-1} \leq j \leq s$. Since $G_{0}, G_{s} \subset I_{n-i+1}$, Lemma 2.3 (2) tells us that there exist at least sixteen intervals $G_{j} \subset I_{n-i}$ with $0 \leq j \leq s$. So there are at least thirteen intervals $G_{j} \subset I_{n-i}$ with $0 \leq j \leq s_{n-i-1}$, and we can define $s_{n-i}$ as in Case (a). Observe that with this definition we still have that there exist at most six intervals $G_{j} \subset I_{n-i}$ with $s_{n-i} \leq j \leq s$.

Case(b'): assume $I_{n-i-1}$ is non-terminating and $I_{n-i}$ is terminating and $i>5$. By the same argument given in the previous case, we know that there exist at most three intervals $G_{j} \subset I_{n-i}$ with $s_{n-i-1} \leq j \leq s$. Since $i+1>4$ there exist at least 16 intervals $G_{j} \subset I_{n-i}$, so at least 13 intervals $G_{j} \subset I_{n-i}$ for $0 \leq j \leq s_{n-i-1}$. This means that we can define $s_{n-i}$ as in Case $(b)$. Observe that with this definition we still have that there exist at most six intervals $G_{j} \subset I_{n-i}$ with $s_{n-i} \leq j \leq s$.

Case(c'): Assume $I_{n-i-1}$ is terminating $I_{n-i} \neq \mathcal{R}\left(I_{n-i-1}\right)$. We have two cases, depending on if $I_{n-i-1}$ is or not periodic.

Case(c'-a): Assume $I_{n-i-1}$ is non-periodic. By the induction hypothesis and the argument given in Case (b'), we know that at least 13 intervals $G_{j} \subset I_{n-i}$ for $0 \leq j \leq s_{n-i-1}$. This means that we can define $s_{n-i}$ as in Case $(c)$. Observe that with this definition we still have that there exist at most six intervals $G_{j} \subset I_{n-i}$ with $s_{n-i} \leq j \leq s$.

Case (c'-b): Assume $I_{n-i-1}=\mathcal{R}\left(I_{n-i-2}\right)$. Then, by Case $(d)$ we know that $s_{n-i-1}=$ $r_{n-i-2}$ is as in Proposition 8.3. By the induction hypothesis there exist at most five intervals $G_{j} \subset I_{n-i}$ with $s_{n-i-1} \leq j \leq s$. Since $i+1>4$ there exist at least 16 intervals $G_{j} \subset I_{n-i}$, so at least 12 intervals $G_{j} \subset I_{n-i}$ for $0 \leq j \leq s_{n-i-1}$. This means that we can define $s_{n-i}$ as in Case $(c)$.

Case(d'): assume $I_{n-i-1}$ is terminating and $I_{n-i}=R\left(I_{n-i-1}\right)$. As in the previous case we need to consider two cases, depending if $I_{n-i-1}$ is or not periodic.

Case( $\left.d^{\prime}-a\right)$ : Assume $I_{n-i-1}$ is non-periodic. Arguing as in the Case $\left(c^{\prime}-a\right)$ we get that there exist at most three intervals $G_{j} \subset I_{n-i}$ with $s_{n-i-1} \leq j \leq s$, and at most two of them are in $I_{n-i+1}$. Since $i+1>4$ there exist at least 8 intervals $G_{j} \subset I_{n-i+1}$, so there exist at least 6 intervals $G_{j} \subset I_{n-i+1}$ for $0 \leq j \leq s_{n-i-1}$. This means that we can define $s_{n-i}$ as in Case $(d)$. Observe that with this definition we still have that there exist at most five intervals $G_{j} \subset I_{n-i+1}$ with $s_{n-i} \leq j \leq s$.

Case(d'-b): Assume $I_{n-i-1}=\mathcal{R}\left(I_{n-i-2}\right)$. By the induction hypothesis, we know that there exist at most five intervals $G_{j} \subset I_{n-i}$ with $s_{n-i-1} \leq j \leq s$, and at most three of them are contained in $I_{n-i+1}$. Since $i+1>4$ there exist at least 8 intervals $G_{j} \subset I_{n-i+1}$, so there exist at least 5 intervals $G_{j} \subset I_{n-i+1}$ for $0 \leq j \leq s_{n-i-1}$. This means that we 
can define $s_{n-i}$ as in Case $(d)$. Observe that with this definition we still have that there exist at most five intervals $G_{j} \subset I_{n-i+1}$ with $s_{n-i} \leq j \leq s$.

So the sequence $s_{n-4} \ldots,<s_{n-M}=s$ is well defined.

Step 3: pulling back. Now, we will make use of the way the times $s_{n-i}$ have been defined to prove (i), (ii) and (iii). For this part of the proof it will be useful to see Figs. 13 and 14. Fix $i \in\{4, \ldots, M-1\}$, and let

$$
U_{s_{n-i-1}}=D_{\theta}\left(I_{n-i-1}\right) \cap \mathbb{C}_{G_{s_{n-i-1}}} \text { and } U_{j}=\operatorname{Comp}_{G_{j}} f^{-\left(s_{n-i-1}-j\right)}\left(U_{s_{n-i-1}}\right),
$$

where $0 \leq j \leq s_{n-i-1}$.

Case ( $\left.a^{\prime}\right)$. By the definition of $s_{n-i}$ we can apply Proposition 8.6 to prove $(i)$.

Case ( $\left.b^{\prime}\right)$. In this case we will prove that (ii) holds. For this we need $s_{n-i-1}, s_{n-i}$ and $s_{n-i+1}$ to be defined, so we assume $i>5$. By definition of $s_{n-i}$ we can apply Proposition 8.2 to find an interval $K^{\prime}$ well-inside $\hat{I}$ and $\lambda^{\prime} \in(0,1)$ so that

$$
U_{s_{n-i}} \subset D_{\lambda^{\prime} \theta}\left(K^{\prime}\right) \text {. }
$$

Since $I_{n-i}$ is terminating $s_{n-i+1} \leq r_{n-i}$ and we can apply Corollary 8.4 to find $\lambda^{\prime \prime}>0$ and an interval $K^{\prime \prime}$ well-inside $\mathcal{R}\left(I_{n-i}\right)$ so that

$$
U_{r_{n-i}} \subset D_{\lambda^{\prime \prime} \theta}\left(K^{\prime \prime}\right) \text {. }
$$

If $I_{n-i+1}=\mathcal{R}\left(I_{n-i}\right)$, then $s_{n-i+1}=r_{n-i}$ and (ii) holds. If $s_{n-i+1}<r_{n-i}$ we apply Proposition 8.5 to get (ii).

Case (c'). if $I_{n-i-1}$ is terminating and $I_{n-i} \neq \mathcal{R}\left(I_{n-i-1}\right)$. Then we can apply Proposition 8.3 to pull back from time $s_{n-i-1}$ to time $r_{n-i-1}$, and Proposition 8.5 to pull back from time $r_{n-i-1}$ to time $s_{n-i}$, so (iii) holds.

Case(d'). By the definition of $s_{n-i}$, (iii) holds from Proposition 8.3 and at most one application of Corollary 4.8 .

From now on, $s_{n-4}<\cdots<s_{n-M}$ will denote the sequence defined in the above proposition and we will assume that $n$ is large.

Lemma 9.2. For each $v \geq 1$ there exists $C>0$ and for each $\theta \in(0, \pi)$ there exists $\varepsilon>0$ such that the following holds. Suppose that $\left|I_{n}\right|<\varepsilon$ and fix $M \in \mathbb{N}$, with $4<M<n$. Assume that for $j=0,1, \ldots, M$

$$
\frac{\left|I_{n-j}\right|}{\left|I_{n-j+1}\right|}<v .
$$

Given $x \in I_{n} \cap \omega\left(c_{0}\right)$ consider the chain $\left\{G_{j}\right\}_{j=0}^{s}$ with $G_{s}=I_{n}$ and $G_{0}=\mathcal{L}_{x}\left(I_{n}\right)$. For $i \in\{4, \ldots, M-1\}$ let

$$
w \in \operatorname{Comp}_{G_{0}} f^{-s_{n-i-1}}\left(V_{n-i-1}\right) \cap \mathbb{C}_{G_{0}} \text { where } V_{n-i-1}:=D_{\theta}\left(I_{n-i-1}\right) \cap \mathbb{C}_{G_{s_{n-i-1}}} .
$$

Then, if $\ell$ is the order of $c_{0}$ the following holds:

(1) if $I_{n-i-1}$ and $I_{n-i}$ are non-terminating, either

$$
w_{s_{n-i}} \in D_{\theta}\left(I_{n-i}\right) \text { or } w \in D_{\theta^{\prime}}\left(I_{n-i}\right),
$$

where $\theta^{\prime}=C \theta\left(\frac{\left|I_{n-i}\right|}{\left|I_{n}\right|}\right)^{\ell-1}$ 
(2) if $I_{n-i-1}$ is non-terminating and $I_{n-i}$ is terminating and $i>5$, then either

$$
w_{s_{n-i+1}} \in D_{\theta}\left(I_{n-i+1}\right) \text { or } w \in D_{\theta^{\prime}}\left(I_{n-i+1}\right),
$$

where $\theta^{\prime}=C \theta\left(\frac{\left|I_{n-i+1}\right|}{\left|I_{n}\right|}\right)^{\ell-1}$

(3) if $I_{n-i-1}$ is terminating, then either

$$
w_{s_{n-i}} \in D_{\theta}\left(I_{n-i}\right) \text { or } w \in D_{\theta^{\prime}}\left(I_{n-i}\right),
$$

where $\theta^{\prime}=C \theta\left(\frac{\left|I_{n-i}\right|}{\left|I_{n}\right|}\right)^{\ell-1}$.

Proof. We will first show the real bounds that will be needed in the proof. By Corollary 3.25, there exists $\delta>0$ so that $I_{n-M}, \ldots, I_{n-1}$ are $\delta$-free. Since we are assuming $\left|I_{n-1}\right|$ is comparable to $\left|I_{n+1}\right|$, Corollary 3.25 or Proposition 3.20 , depending on the definition of $I_{n}$, imply that $I_{n}$ is $\delta$-free except if $I_{n}=\mathcal{R}\left(I_{n-1}\right)$ and it is periodic; in this case the we need $\left|I_{n-1}\right|$ to be comparable to $\left|I_{n+2}\right|$ to conclude that $I_{n}$ is $\delta$-free, which we do not have. In this particular case, we will use a slightly different argument involving the interval $I_{n-1}$ and its pullbacks, instead of the intervals $G_{j}$. To do so we will define a new chain as follows. If $I_{n}=\mathcal{R}\left(I_{n-1}\right)$ and it is periodic let $\left\{\hat{G}_{j}\right\}_{j=0}^{s}$ be the chain with $\hat{G}_{s}=I_{n-1}$ and $\hat{G}_{j}=\operatorname{Comp}_{G_{j}} f^{-1}\left(\hat{G}_{j+1}\right)$ for $0 \leq j<s-1$. Note that the intersection multiplicity of this chain is bounded by two, since the chain $\left\{G_{j}\right\}_{j=0}^{s-1}$ is disjoint and $I_{n}$ has period two inside $I_{n-1}$. By Corollary 3.5, there exists $\hat{\delta}>0$ so that if $G_{s}=I_{n}$ is $\delta$-free, then $G_{j}$ is $\hat{\delta}$-free for all $0 \leq j \leq s$, and if $\hat{G}_{s}$ has been defined, then $\hat{G}_{j}$ is $\hat{\delta}$-free for all $0 \leq j \leq s$, since $\hat{G}_{s}=I_{n-1}$ is $\delta$-free. Finally, by Theorem 3.1 we know that if $I_{n-i-1}$ is non-terminating, then it is $\hat{\rho}$-nice for some $\hat{\rho}>0$.

Step 1: Pulling back to an interval well-inside an interval in the enhanced nest. Let $U_{j}=\operatorname{Comp}_{G_{j}} f^{-\left(s_{n-i-1}-j\right)}\left(V_{n-i-1}\right)$ for $0 \leq j \leq s_{n-i-1}$. We will study each of the cases from the statement separately.

(1) If $I_{n-i-1}$ and $I_{n-i}$ are non-terminating. By Proposition 9.1 there exists $\mu \in(0,1)$ and an interval $K$ well-inside $I_{n-i}$ such that $U_{s_{n-i}} \subset D_{\mu \theta}(K)$, where $K=\mathcal{L}_{G_{s_{n-i}}}\left(I_{n-i}\right)$ and $G_{s_{n-i}} \subset K$. In this case, let $I_{n-i}^{f}$ be the pullback of $I_{n-i-1}$ under $f^{p_{n-i-1}-1}$ containing $f\left(I_{n-i}\right)$. In this case define $i_{0}=n-i$ and $s^{*}=s_{n-i}$.

(2) If $I_{n-i-1}$ is non-terminating and $I_{n-i}$ is terminating and $i>5$. By Proposition 9.1 there exists $\mu \in(0,1)$ and an interval $K$ well-inside $I_{n-i+1}$ such that $U_{s_{n-i+1}} \subset$ $D_{\mu \theta}(K)$, with $G_{s_{n-i+1}} \subset K$. Assume the return time of $c_{0}$ to $\mathcal{R}\left(I_{n-i}\right)$ is equal to $q$ and let $I_{n-i+1}^{f}$ be the pullback of $\mathcal{R}\left(I_{n-i}\right)$ under $f^{q-1}$ containing $f\left(I_{n-i+1}\right)$. In this case define $i_{0}=n-i+1$ and $s^{*}=s_{n-i+1}$.

(3) If $I_{n-i-1}$ is terminating we have two cases.

(a) If $I_{n-i} \neq \mathcal{R}\left(I_{n-i-1}\right)$. By Proposition 9.1 there exists $\mu \in(0,1)$ and an interval $K$ well-inside $I_{n-i}$ such that $U_{s_{n-i}} \subset D_{\mu \theta}(K)$, with $G_{s_{n-i}} \subset K$. Assume the return time of $c_{0}$ to $\mathcal{R}\left(I_{n-i-1}\right)$ is equal to $q$ and let $I_{n-i}^{f}$ be the pullback of $\mathcal{R}\left(I_{n-i-1}\right)$ under $f^{q-1}$ containing $f\left(I_{n-i}\right)$. In this case define $i_{0}=n-i$ and $s^{*}=s_{n-i}$.

(b) If $I_{n-i}=\mathcal{R}\left(I_{n-i-1}\right)$. By Proposition 9.1 there exists $\mu \in(0,1)$ and an interval $K$ well-inside $I_{n-i}$ such that $U_{s_{n-i}} \subset D_{\mu \theta}(K)$, with $G_{s_{n-i}} \subset K$. Assume the return time of $c_{0}$ to $I_{n-i}$ is equal to $q$ and let $I_{n-i}^{f}$ be the pullback of $I_{n-i}$ under $f^{q-1}$ containing $f\left(I_{n-i}\right)$. In this case define $i_{0}=n-i$ and $s^{*}=s_{n-i}$. 
Observe that, independently of the case, the definition of $i_{0}$ and $s^{*}$ give the following:

$$
U_{s^{*}} \subset D_{\mu \theta}(K)
$$

and $G_{s^{*}} \subset K \subset I_{n-i_{0}}$. We always have that

$$
\operatorname{Comp}_{c_{0}} f^{-1}\left(I_{i_{0}}^{f}\right)=I_{n-i_{0}} .
$$

We know that $I_{n-i_{0}-1}$ is $\delta$-free. If $I_{n-i_{0}-1}$ is terminating, Proposition 3.20 tells us that there exists $\delta^{\prime}>0$ so that $\mathcal{R}\left(I_{n-i_{0}-1}\right)$ is $\delta^{\prime}$-free. So shrinking $\delta$, if necessary, we can assume that if $I_{n-i_{0}-1}$ is terminating, $I_{n-i_{0}-1}$ and $\mathcal{R}\left(I_{n-i_{0}-1}\right)$ are $\delta$-free. By definition, $I_{n-i_{0}}^{f}$ is a pullback of bounded order of a $\delta$-free interval so, regardless of the definition of $i_{0}$, the interval $I_{n-i_{0}}^{f}$ is $\delta^{\prime \prime}$-free for some $\delta^{\prime \prime}>0$. In the remainder of the proof $C_{k}$, for $k \in \mathbb{N}$, stands for a positive constant that depends only on the previous constants. Observe that, in any case, if $w_{s^{*}} \in D_{\theta}\left(I_{n-i_{0}}\right)$ we are done with the proof of the proposition, so let us assume $w_{s^{*}} \notin D_{\theta}\left(I_{n-i_{0}}\right)$. Then

$$
w_{s^{*}} \in D_{\mu \theta}(K) \backslash D_{\theta}\left(I_{n-i_{0}}\right) .
$$

By Lemma 4.5 we have that there exists $C_{1}>0$ so that

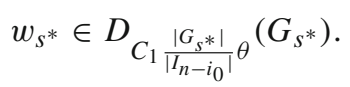

Step 2: Pulling back to the start. If $I_{n-i_{0}}$ is non-periodic, then by Proposition 9.1 we know that $f^{s-s^{*}}: G_{s^{*}} \rightarrow G_{s}=I_{n}$ is at most a sixth iterate of the first return map $R_{I_{n-i_{0}}}$. So there exists $\nu_{0}>0$ so that $\left|G_{s^{*}}\right| /\left|I_{n}\right| \geq v_{0}$. Indeed, if $\left|G_{s^{*}}\right| /\left|I_{n}\right|$ is small, then the derivative of $R_{I_{n-i}}$ would be big at some point. Then, since $I_{n-i_{0}}$ is $\delta$-free Theorem 3.2 would imply that one of the components of the domain of $R_{I_{n-i_{0}}}$ is small compared to $\left|I_{n-i_{0}}\right|$. Theorem 3.1 or Lemma 3.10, depending on whether $I_{n-i_{0}}$ is nonterminating or terminating, allows us to conclude that $I_{n-i_{0}+1}$ is small compared to $I_{n-i_{0}}$, which is a contradiction. If $I_{n-i_{0}}$ is periodic, then by Proposition 9.1 we know that $f^{s-s^{*}}: G_{s^{*}} \rightarrow G_{s}=I_{n}$ is at most a fifth iterate of the first return map $R_{I_{n-i_{0}+1}}$, so we conclude that $\left|G_{s^{*}}\right| /\left|I_{n}\right| \geq v_{0}$ using the same argument as before. Similarly, if $I_{n}=\mathcal{R}\left(I_{n-1}\right)$ and it is periodic, we have that $\left|\hat{G}_{S^{*}}\right| /\left|I_{n-1}\right| \geq v_{0}$.

Assume $I_{n} \neq \mathcal{R}\left(I_{n-1}\right)$. Then, since $G_{s^{*}}$ is $\delta$-free and the intervals $G_{0}, \ldots, G_{s^{*}}$ are disjoint we have that

$$
w_{1} \in D_{C_{2} \frac{\left|G_{s^{*}}\right|}{\left|I_{n-i_{0}}\right|} \theta}\left(G_{1}\right) .
$$

Because $\left|G_{s^{*}}\right| /\left|I_{n}\right| \geq \kappa_{0}$, we have

$$
w_{1} \in D_{C_{3} \frac{\left|I_{n}\right|}{\left|I_{n-i_{0} \mid}\right|} \theta}\left(G_{1}\right) .
$$

By Lemma 4.5 we have that

$$
w_{1} \in D \underset{C_{4} \frac{\left|I_{n}\right|}{\left|I_{n-i_{0}}\right|} \mid \frac{\left|I_{n-i_{0} \mid}^{f}\right|}{\left|G_{1}\right|} \theta}{f}\left(I_{n-i_{0}}^{f}\right) .
$$


Since $I_{n-i_{0}}^{f}$ is $\delta^{\prime \prime}$-free, Lemma 4.2 gives us that

$$
w \in C_{5 \frac{\left|I_{n}\right|}{\left|I_{n-i_{0}}\right|} \frac{\left|I_{n-i_{0} \mid}^{f}\right|}{\left|G_{1}\right|} \theta}\left(I_{n-i_{0}}\right) .
$$

Note that $G_{0} \subset I_{n}$, and therefore $\left|I_{n}\right|^{\ell} /\left|G_{1}\right| \geq C_{6}$. Finally, since $I_{n-i_{0}}$ is $\delta$-free we have that $\left|I_{n-i_{0}}^{f}\right| \geq C_{7}\left|I_{n-i_{0}}\right|^{\ell}$. Hence



Assume $I_{n}=\mathcal{R}\left(I_{n-1}\right)$ and it is periodic. Then using the same argument as above, substituting $G_{j}$ by $\hat{G}_{j}$ and $I_{n}$ by $I_{n-1}$ we have the following:

$$
w \in C_{8} \frac{\left|I_{n-i_{0}}\right|^{\ell-1}}{\left|I_{n-1}\right|^{\ell-1}} \theta\left(I_{n-i_{0}}\right) .
$$

Now, since $\left|I_{n}\right|>v\left|I_{n-1}\right|$ there exists $C_{9} \in(0,1)$ so that

$$
w \in D_{C_{9}} \frac{\mid I_{n-i_{0}} \ell^{\ell-1}}{\left|I_{n}\right|^{\ell-1}} \theta\left(I_{n-i_{0}}\right) .
$$

Taking $C$ as the minimum between $C_{8}$ and $C_{9}$ the proposition follows.

\subsection{The construction of a qr quasi-box mapping.}

Theorem 9.3 (The existence of a quasi-box mapping). For each $v \geq 1$ there exists $M>0$, and for each $\theta \in(0, \pi / 2]$ there exists $\varepsilon>0$ so that, if $\left|I_{n-M}\right|<\varepsilon$ the following holds. If for all $j \in\{n-M, \ldots n+1\}$ we have that

$$
\left|I_{j-1}\right| /\left|I_{j}\right| \leq v,
$$

then the first return mapping to $I_{n}$ extends to a quasi-box mapping with range $D_{\theta}\left(I_{n-M}\right)$. Furthermore, there exists a universal constant $v_{0} \geq 1$ so that, if $\left|I_{j-1}\right| /\left|I_{j}\right|>v_{0}$, for $j$ sufficiently large, then we obtain a quasi-box mapping extending the return map to a puzzle piece $\hat{I}$, with $I_{j+2} \subset \hat{I} \subset I_{j-1}$.

Remark. See the corollary following the proof of the theorem for a more precise statement in the non-renormalizable case.

Let us recall that the domains of the qr quasi-box mapping may intersect each other and that they do not have to be compactly contained in the range.

Proof. We will divide the proof of the theorem in steps. In this proposition when we talk about first return maps to an interval $I$ containing $c_{0}$, we will be referring to the first return map to $I$ restricted to the components of $R_{I}$ that intersect $\omega\left(c_{0}\right)$. In what follows, assume that all intervals $I_{j}$ from the generalized enhanced nest have $j$ large enough so that $\left|I_{j}\right|<\varepsilon$, for all $\varepsilon$ in this section and in Sect. 8 .

Step 1. Let $v \geq 1$ and let $C$ be the constant associated to $v$ by Lemma 9.2. Choose an integer $M$, with $4<M$ so that for all $n$ sufficiently large

$$
D_{\mu^{3} \theta}\left(I_{n-4}\right) \subset D_{\theta}\left(I_{n-M+1}\right),
$$


where $\mu \in(0,1)$ is the constant from Proposition 9.1, and so that for each $i=n-$ $M, \ldots, n-1$ either

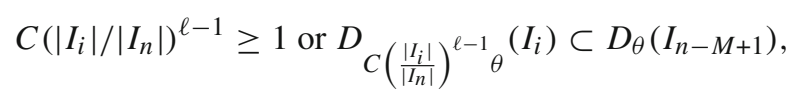

where $\ell$ is the order of $c_{0}$. To see that such $M$ exists notice that because of real bounds there exists $\rho>0$ so that $(1+2 \rho) I_{j} \subset I_{j-1}$, for every $j \in \mathbb{N}$. So we can take $M$ large enough, so that $(M+1) \rho>1 / C$.

Assume that for all $j \in\{n-M, \ldots n+1\}$,

$$
\left|I_{j-1}\right| /\left|I_{j}\right| \leq \nu \text {. }
$$

Let $x \in \omega\left(c_{0}\right) \cap I_{n}$ and let $s>0$ be minimal so that $f^{s}(x) \in I_{n}$. Let $\left\{G_{j}\right\}_{j=0}^{s}$ be the chain with $G_{s}=I_{n}$ and $G_{0}=\mathcal{L}_{x}\left(I_{n}\right)$. Let $s_{n-4}<\cdots<s_{n-M-1}<s_{n-M}:=s$ be the sequence defined in Proposition 9.1. Let

$$
w \in \operatorname{Comp}_{G_{0}} f^{-s_{n-M}}\left(V_{n-M}\right) \cap \mathbb{C}_{G_{0}} \text { where } V_{n-M}:=D_{\theta}\left(I_{n-M}\right) \cap \mathbb{C}_{G_{s_{n-i-1}}}
$$

and define $w_{s_{n-i}}=f^{s_{n-i}}(w)$ for $i \in\{4, \ldots, M-1\}$. By Lemma 9.2 we know that one of the following holds:

(1) If $I_{n-M}$ and $I_{n-M+1}$ are non-terminating, either

$$
w_{s_{n-M+1}} \in D_{\theta}\left(I_{n-M+1}\right) \text { or } w \in D_{\theta^{\prime}}\left(I_{n-M+1}\right),
$$

where $\theta^{\prime}=C \theta\left(\frac{\left|I_{n-M+1}\right|}{\left|I_{n}\right|}\right)^{\ell-1}$.

(2) If $I_{n-M}$ is non-terminating and $I_{n-M+1}$ is terminating, then either

$$
w_{s_{n-M+2}} \in D_{\theta}\left(I_{n-M+2}\right) \text { or } w \in D_{\theta^{\prime}}\left(I_{n-M+2}\right),
$$

where $\theta^{\prime}=C \theta\left(\frac{\left|I_{n-M+2}\right|}{\left|I_{n}\right|}\right)^{\ell-1}$;

(3) If $I_{n-M}$ is terminating either

$$
w_{s_{n-M+1}} \in D_{\theta}\left(I_{n-M+1}\right) \text { or } w \in D_{\theta^{\prime}}\left(I_{n-M+1}\right),
$$

where $\theta^{\prime}=C \theta\left(\frac{\left|I_{n-M+1}\right|}{\left|I_{n}\right|}\right)^{\ell-1}$;

By the choice of $M$, if the second assertion holds, on the corresponding case, we get that $w \subset D_{\theta}\left(I_{n-M}\right) \cap \mathbb{C}_{I_{n}}$, and the theorem holds. If the first assertion holds, we repeat the argument. If after at most $M-3$ repetitions we have not proved the theorem we get

$$
w_{s_{n-4}} \in D_{\theta}\left(I_{n-4}\right), \text { where } \theta^{\prime}=C \theta\left(\frac{\left|I_{n-4}\right|}{\left|I_{n}\right|}\right)^{\ell-1} .
$$

We know $\left|I_{i-2}\right| /\left|I_{i+1}\right|<v_{0}$ for $i \in\{n-4, \ldots, n-1\}$. So we can follow the corresponding proofs in Sect. 8 to control the loss of angle and obtain

$$
w \in D_{\mu^{3} \theta}\left(I_{n-4}\right) .
$$

For example, see the proof of Theorem 10.18. 
Step 2. Now we show that there exists a constant $v_{0} \geq 1$, such that if $\left|I_{j-1}\right| /\left|I_{j}\right|>v_{0}$, for some $j \in\{n-M, \ldots n+1\}$ then we obtain a complex extension for a return map to a puzzle piece $\hat{I}$, with

$$
I_{j+2} \subset \hat{I} \subset I_{j-1} .
$$

Let $v_{0}$ be the maximum of the constants $v_{1}$ from Sect. 8.3. Assume that $\left|I_{j-1}\right| /\left|I_{j}\right|>v_{0}$ for $j \in\{n-M, \ldots, n+1\}$.

Case (a): If $I_{j-1}$ and $I_{j}$ are non-terminating. By Proposition 8.7 we know that the return mapping to $I_{j+1}$ extends to a qr box mapping with range $D_{\pi / 2}\left(I_{j+1}\right)$.

Case (b): If $I_{j-1}$ is non-terminating and $I_{j}$ is terminating. By Corollary 8.10 the first return map to $I_{j}^{\infty}$ extends to a qr box mapping with range $D_{\pi / 2}\left(I_{j}^{2}\right)$.

Case (c): If $I_{j-1}$ is terminating. By Proposition 8.11 one of the following holds: the first return map to $I_{j-1}^{\infty}$ extends to a qr box mapping with range $D_{\pi / 2}\left(I_{j-1}^{2}\right) ; I_{j}$ is terminating and the first return map to $I_{j}^{\infty}$ extends to a qr box mapping with range $D_{\pi / 2}\left(I_{j}^{2}\right) ; I_{j}$ and $I_{j+1}$ are non-terminating and the first return map to $I_{j+2}$ extends to a qr complex box mapping with range $D_{\pi / 2}\left(I_{j+2}\right) ; I_{j}$ is non-terminating and $I_{j+1}$ is terminating the first return map to $I_{j+1}^{\infty}$ extends to a qr box mapping with range $D_{\pi / 2}\left(I_{j+1}^{2}\right)$. that $\left|I_{j-1}\right| /\left|I_{j}\right|>v_{0}$, we can construct a qr quasi-box mapping as in the statement of the theorem.

Corollary 9.4 (Quasi-box mapping in the non-renormalizable case). Suppose that $f$ is non-renormalizable. Given $v \geq 1$, there exists $M>0$ with the following properties. For $\theta \in(0, \pi / 2]$, if $n \in \mathbb{N}$ is sufficiently large:

(1) If $v \geq v_{0}$, where $v_{0}$ is the constant from Proposition 8.7, and $\left|I_{k}\right| /\left|I_{k+1}\right|>v_{0}$ for some $k \in\{n, n-1, \ldots, n-M\}$ then: the first return mapping to $I_{k+2}$ extends to a qr box mapping $F: \mathcal{U} \rightarrow \mathcal{V}$ with range $\mathcal{V}=D_{\pi / 2}\left(I_{k+2}\right)$ and $\mathcal{U} \subset D_{\theta}\left(I_{k+2}\right)$.

(2) If for all $k \in\{n, n-1, \ldots, n-M\}$ we have that $\left|I_{k}\right| /\left|I_{k+1}\right| \leq v$, then the first return mapping to $I_{n}$ extends to a qr quasi-box mapping $F: \mathcal{U} \rightarrow \mathcal{V}$ with range $\mathcal{V}=D_{\theta}\left(I_{n-M}\right) \cap \mathbb{C}_{I_{n}}$ and $\mathcal{U} \subset D_{\theta}\left(I_{n-M+1}\right)$.

This concludes the construction of the qr quasi-box mapping.

\section{Box Mappings and Complex Bounds}

In this section, we make use of the qr quasi-box mappings from Theorem 9.3 to construct qr box mappings (this notion was introduced in Sect. 5.3). In particular, when $f$ is real analytic the box mappings we obtain are holomorphic.

As usual, for a map $f \in \mathcal{A}_{b}^{3}$ we abuse notation and denote by $f$ its asymptotically holomorphic extension of order 3 , given in Sect. 5.2.

10.1. Finitely renormalizable maps. We follow similar arguments to those of Levin-van Strien in [LvS2], which were also used in [S3]. However, we augment them to show that we can obtain complex bounds (i.e. bounds for the moduli of relevant annuli) for the qr box mappings. Throughout this subsection we assume that $f: M \rightarrow M$ is at most finitely many times renormalizable. 
As an intermediate step in our construction of qr box mappings we will make use of smooth box mappings, which are maps of the form $F_{S}: \mathcal{U}_{S} \rightarrow \mathcal{V}_{S}$, which have all the properties of a complex box mapping (see p. 2) except that we only require $F$ to be $C^{3}$.

Let $N>0$ be maximal so that none of the intervals $I_{N-i}$ with $i=0,1,2,3$ is terminating. For ease of exposition, assume $N=0$, and that all of the intervals $I_{0} \supset$ $I_{1} \supset I_{2} \supset \cdots$ of the enhanced nest about $c_{0}$ are non-terminating.

If $I$ is a nice interval containing $c_{0}$, we let

$$
\hat{I}=\bigcup_{c \in \operatorname{Crit}(f) \cap \omega\left(c_{0}\right)} \hat{\mathcal{L}}_{c}(I)
$$

and let $\mathrm{D}(\hat{I})$ denote the union of all first return domains to $\hat{I}$ that intersect $\omega\left(c_{0}\right)$. We denote the first return map to $\hat{I}$ by

$$
R_{\hat{I}}: D(\hat{I}) \rightarrow \hat{I}
$$

Our aim is to prove the following:

Theorem 10.1 (Complex bounds in the non-renormalizable case). There exists $\delta>0$ such that the following holds. Suppose that

$$
R_{\hat{I}_{0}}: \mathrm{D}\left(\hat{I}_{0}\right) \rightarrow \hat{I}_{0}
$$

is non-renormalizable (at $\left.c_{0}\right)$. Then for all $m$ sufficiently large, there exists a qr box mapping $F: \mathcal{U} \rightarrow \mathcal{V}$ extending $R_{\hat{I}_{m}}$ with the property that $\mathcal{V}$ is $\delta$-nice, $\delta$-free and $\mathcal{U}$ has $\delta$-bounded geometry.

The proof of the theorem above will occupy the following proposition and the next two subsections.

Proposition 10.2 (cf. [LvS2], pp. 425-427). Given $v \geq 1$, there exists $\varepsilon>0, m \in \mathbb{N}$, $\eta \geq 1$ and $\theta \in(0, \pi)$ such that the following holds. Suppose that

$$
R_{\hat{I}_{0}}: \mathrm{D}\left(\hat{I}_{0}\right) \rightarrow \hat{I}_{0}
$$

is non-renormalizable at $c_{0}$. Then given $n \in \mathbb{N}$ with $\left|I_{n-m}\right|<\varepsilon$ and so that $\left|I_{j-1}\right| /\left|I_{j}\right| \leq$ $\nu$, for all $j \in\{n-m+1, \ldots n+1\}$ the real return mapping $R_{\hat{I}_{n}}$ extends to a qr box mapping $F: \mathcal{U} \rightarrow \mathcal{V}$ with the following properties: the map $\left.F\right|_{U}$ is at most unicritical for all $U \in \mathcal{U}$; if $V$ is a component of $\mathcal{V}$, then $\operatorname{diam}(V) \leq \eta|V \cap \mathbb{R}|$ and $D_{\theta}(V \cap \mathbb{R}) \subset V$.

Proof. Let $M$ be the constant associated to $v$ by Corollary 9.4. We will choose the constant $m>M$ in the course of the proof; $M$ will be independent of $n$. How small $\left|I_{n-m}\right|$ needs to be will be determined by the proof. As usual, let $p_{n} \in \mathbb{N}$ be so that $I_{n+1}=\operatorname{Comp}_{c_{0}} f^{-p_{n}}\left(I_{n}\right)$. By Theorem 3.1, the intervals $I_{n}$ are $\delta>0$ externally and internally free, for some $\delta=\delta(v)$, and each component of the internal free space is of size comparable to $\left|I_{n}\right|$.

Claim. Given $\delta_{1}>0$, there exists $\varepsilon_{1}>0$ and $\theta_{1} \in(\pi / 2, \pi)$ such that the following holds. Suppose $I$ is a $\delta_{1}$-externally free nice interval, with $I \backslash\left(1+2 \delta_{1}\right)^{-1} I$ disjoint form $\omega\left(c_{0}\right)$ and $|I|<\varepsilon_{1}$. Let $V=D_{\theta_{1}}(I)$ and consider $G_{0}^{1}=\mathcal{L}_{x}(I)$ and $G_{0}^{2}=\mathcal{L}_{y}(I)$ disjoint, with $x, y \in \omega\left(c_{0}\right)$. Let $U_{0}^{1}$ and $U_{0}^{2}$ be the components of the landing map to $V$ that contain $G_{0}^{1}$ and $G_{0}^{2}$, respectively. Then $U_{0}^{1}$ and $U_{0}^{2}$, are disjoint. 
Proof of claim. Let $F$ be the interval that defines the external free space of $I$. Consider the chains $\left\{G_{j}^{i}\right\}_{j=0}^{s_{i}}$ associated to $G_{0}^{1}$ and $G_{0}^{2}$, so $G_{s_{i}}^{i}=I$, and let $\left\{H_{j}^{i}\right\}_{j=0}^{s_{i}}$ be the corresponding chains associated to the pullbacks of $F$, for $i=1,2$. Let $U_{j}^{i}=\operatorname{Comp}_{G_{j}^{i}} f^{s_{i}-j}(V)$ for $j=0, \ldots s_{i}$. If the order the chains $\left\{G_{j}^{i}\right\}_{j=0}^{s_{i}}$ is zero, then provided that $\theta_{1} \in(0, \pi)$ is chosen sufficiently close to $\pi$, and $\varepsilon_{1}$ is small enough, Corollary 5.7 implies that $U_{0}^{1}$ and $U_{0}^{2}$ are contained in the geometric circles based on their real traces, so the claim follows. Otherwise, let $s<\min \left\{s_{1}, s_{2}\right\}$ be maximal so that either $G_{s}^{1}$ or $G_{s}^{2}$ contains a critical point. By Lemma 4.6, we can choose $\theta_{1} \in(\pi / 2, \pi)$ so that $U_{s}^{1} \cap U_{s}^{2}=\emptyset$. Observe that this can be done provided $\varepsilon_{1}$ is sufficiently small. From this it follows that $U_{0}^{1} \cap U_{0}^{2}=\emptyset$.

Let $\theta_{1}=\theta_{1}(\delta)$ and $\varepsilon_{1}(\delta)$ be the constants given by the claim, and assume $\left|I_{n-m}\right|<\varepsilon_{1}$. Step 1: Construction of a smooth box mapping at level $I_{n-m}$.

There exists $\theta_{2} \in\left(\theta_{1}, \pi\right)$, depending only on $\delta$ and a smooth box mapping $F_{S}: \mathcal{U}_{S} \rightarrow$ $\mathcal{V}_{S}$ with the following properties:

- The map $F_{s}$ extends $R_{\hat{I}_{n-m}}: \mathrm{D}\left(\hat{I}_{n-m}\right) \rightarrow \hat{I}_{n-m}$ in a neighbourhood of $\mathrm{D}\left(\hat{I}_{n-m}\right)$, it is asymptotically holomorphic and has the same critical points as $R_{\hat{I}_{n-m}}$;

- for each $U \in \mathcal{U}_{S},\left.F\right|_{U}$ is at most unicritical;

- for each $U \in \mathcal{U}_{S}, D_{\theta_{2}}(U \cap \mathbb{R}) \subset U$ and $F_{S}=f^{r}$ on $D_{\theta_{2}}(U \cap \mathbb{R})$, where $r$ is the return time of $U$ to $\mathcal{V}$ under $f$.

Step 1a: Obtaining a complex extension such that the components of its domain are disjoint. We begin by constructing an intermediate smooth mapping $\tilde{F}_{S}: \tilde{\mathcal{U}}_{S} \rightarrow \tilde{\mathcal{V}}_{S}$ where $\tilde{\mathcal{V}}_{S}$ is a neighbourhood of $\operatorname{Crit}(f) \cap \omega\left(c_{0}\right)$ and the components of $\tilde{\mathcal{U}}_{S}$ are disjoint, but not necessarily contained in $\tilde{\mathcal{V}}_{S}$.

Let $V_{c_{0}}=D_{\theta_{1}}\left(I_{n-m}\right)$. For each $c \in \operatorname{Crit}(f) \cap \omega\left(c_{0}\right)$, let $V_{c}=\hat{\mathcal{L}}_{c}\left(V_{c_{0}}\right)$, and let

$$
\tilde{\mathcal{V}}_{S}=\bigcup_{c \in \operatorname{Crit}(f) \cap \omega\left(c_{0}\right)} V_{c} .
$$

Observe that $\tilde{\mathcal{V}}_{S} \cap \mathbb{R}=\hat{I}_{n-m}$. For each $x \in \omega\left(c_{0}\right) \cap \hat{I}_{n-m}$, let $\tilde{U}(x)=\mathcal{L}_{x}\left(\tilde{\mathcal{V}}_{s}\right)$ and let

$$
\tilde{\mathcal{U}}_{S}=\bigcup_{x \in \omega\left(c_{0}\right) \cap \hat{I}_{n-m}} \tilde{U}_{x} .
$$

Notice that if $x \in \omega\left(c_{0}\right) \cap \hat{\mathcal{L}}_{c}\left(I_{n-m}\right)$ for $c \neq c_{0}$, then $\tilde{U}(x)=\tilde{V}_{c}$. The claim implies that the components of $\tilde{\mathcal{V}}_{S}$ are pairwise disjoint, and that the components of $\tilde{\mathcal{U}}_{S}$ together with the components of $\tilde{\mathcal{V}}_{S} \backslash V_{c_{0}}$ are pairwise disjoint. By making $\varepsilon_{1}$ smaller, if necessary, Lemma 4.2, Corollary 5.6, Lemma 4.3 and Corollary 5.7, imply that there exist $\theta_{2} \in$ $\left(\theta_{1}, \pi\right)$ and $\theta_{2}^{\prime} \in(0, \pi)$, depending only on $\delta$ and $\underline{b}$, with the following property. For each component $U$ of $\tilde{\mathcal{U}}_{S}$ or of $\tilde{\mathcal{V}}_{S}$,

$$
D_{\theta_{2}}(U \cap \mathbb{R}) \subset U \subset D_{\theta_{2}^{\prime}}(U \cap \mathbb{R}) .
$$

Define a mapping $\tilde{F}_{S}: \tilde{\mathcal{U}}_{S} \rightarrow \tilde{\mathcal{V}}_{S}$ as follows, for each component $U$ of $\tilde{\mathcal{U}}_{S}$, let $s>0$ denote its first entry time to $\tilde{\mathcal{V}}_{S}$ under $f$, so that $f^{s}(U)$ is a component $V$ of $\tilde{\mathcal{V}}_{S}$. Set $\tilde{F}_{S}\left|U=f^{s}\right| U$. 
Step 1b. Obtaining a smooth box mapping. For each critical point $c \in \omega\left(c_{0}\right)$, let $A_{c}$ be topological disk that properly contains $\tilde{V}_{c}$. We can choose these disks so that $\left\{A_{c}: c \in\right.$ $\left.\omega\left(c_{0}\right) \cap \operatorname{Crit}(f)\right\}$ is a collection of domains with pairwise disjoint closures such that given $x \in \omega\left(c_{0}\right) \cap \hat{I}_{n-m}$, if $\tilde{U}(x)$ intersects $A_{c}$ and $\tilde{U}(x) \neq \tilde{V}_{c}$, then $\tilde{U}(x)$ is compactly contained in $A_{c}$. For each such $\tilde{U}(x)$ let $B(x)$ be a topological disk such that $\tilde{U}(x) \Subset$ $B(x) \Subset A_{c}$. Moreover, choose the domains $B(x)$ so that they have pairwise disjoint closures. For each $\tilde{U}(x)$, let $c$ be such that $\tilde{V}_{c}=\tilde{F}_{S}(\tilde{U}(x))$ and extend $\tilde{F}_{S}: \tilde{U}(x) \rightarrow \tilde{V}_{c}$ to a smooth map $F_{S} \mid B(x) \rightarrow A_{c}$. Let

$$
\mathcal{V}_{S}=\bigcup_{c \in \omega\left(c_{0}\right) \cap \operatorname{Crit}(f)} A_{c} \text { and } \mathcal{U}_{S}=\left(\bigcup_{x \in \omega\left(c_{0}\right)} B(x)\right) \cup\left(\bigcup_{c \in \omega\left(c_{0}\right) \cap \operatorname{Crit}(f) \backslash\left\{c_{0}\right\}} A_{c}\right) \text {. }
$$

Then

$$
F_{S}: \mathcal{U}_{S} \rightarrow \mathcal{V}_{S}
$$

is a smooth box mapping with the required properties. This concludes Step 1.

From now on, $\theta_{2}$ is the constant given by Step 1 .

Step 2: Choosing $m$. We will choose $m$ so that, roughly, the domain of the quasi-box mapping given by Corollary 9.4 that extends the return map to $I_{n}$ is contained in the part of the plane, close to the real line, where $F_{S}$ agrees with the corresponding iterate of $f$.

From Corollary 9.4 we know there exists $M \in \mathbb{N}$, depending on $v$, so that the first return map to $I_{n}$ extends to a quasi-box mapping with range $V_{Q}=D_{\pi / 2}\left(I_{n-M}\right) \cap \mathbb{C}_{I_{n}}$. For each critical point $c \in \operatorname{Crit}(f) \cap \omega\left(c_{0}\right), c \neq c_{0}$, let $r_{c}>0$ be minimal so that $f^{r_{c}}(c) \in I_{n}$. Set $\hat{V}_{c_{0}}=V_{Q}$ and $\hat{V}_{c}=\operatorname{Comp}_{c} f^{-r_{c}}\left(\hat{V}_{c_{0}}\right)$. Since $\left|I_{j-1}\right| /\left|I_{j}\right|<v$, for all $n-1 \leq j \leq n-M+1$, there exists $\theta_{3} \in(0, \pi)$ so that

$$
\hat{V}_{c} \subset D_{\theta_{3}}\left(\hat{\mathcal{L}}_{c}\left(I_{n-M}\right)\right) \cap \mathbb{C}_{\hat{\mathcal{L}}_{c}\left(I_{n}\right)} .
$$

To see this, choose any $\hat{V}_{c}$ with $c \neq c_{0}$. There exists $x \in \omega\left(c_{0}\right) \cap I_{n}$ such that if $s>0$ is minimal so that $f^{s}(x) \in I_{n}$, then there exists $0<r_{x}<s$ so that $f^{r_{x}}(x) \in \mathcal{L}_{c}\left(I_{n}\right)$. Observe that the landing times of $f^{r_{x}}(x)$ and $c$ to $I_{n}$ under $f$ are the same; they are both $r_{c}=s-r_{x}$. Let $\left\{G_{j}\right\}_{j=0}^{s}$ be the chain with $G_{s}=I_{n}$ and $G_{0}=\mathcal{L}_{x}\left(I_{n}\right)$, and let

$$
s_{n-4}<\cdots<s_{n-M}:=s
$$

be the sequence given by Proposition 9.1. Let $w \in \operatorname{Comp}_{x} f^{-s} D_{\pi / 2}\left(I_{n-M}\right)$ and $w_{i}=$ $f^{i}(w)$ for $i \in \mathbb{N}$. Either there exists $s_{n-j}$ so that $s_{n-j+1}<r_{x}<s_{n-j}$ or $0<r_{x}<s_{n-4}$. Let us assume $s_{n-j+1}<r_{x}<s_{n-j}$. By Proposition 9.1, there exists $\mu \in(0,1)$ such that $w_{s_{n-j}} \in D_{\mu \pi / 2}\left(\mathcal{L}_{G_{s_{n-j}}}\left(I_{n-j}\right)\right) \subset I_{n-j}$. By Lemma 8.1, we know $k\left(I_{n-j},\left\{G_{j}\right\}_{j=r_{x}}^{s_{n-j}}\right)$ is bounded. This and the fact that $I_{n-j}$ is $\delta$-nice, allow us to apply Proposition 7.1 and Corollary 4.8 to find $\theta_{3} \in(0, \pi)$ so that $w_{r_{x}} \in D_{\theta_{3}}\left(\hat{\mathcal{L}}_{c}\left(I_{n-j}\right)\right)$. The proof when $0<r_{x}<s_{n-4}$ is analogous to this one.

Now choose $m$ so that for each critical point $c \in \operatorname{Crit}(f) \cap \omega\left(c_{0}\right)$

$$
D_{\theta_{3}}\left(\hat{\mathcal{L}}_{c}\left(I_{n-M+1}\right)\right) \subset D_{\theta_{2}}\left(\hat{\mathcal{L}}_{c}\left(I_{n-m}\right)\right) .
$$

Step 3. Intersecting the smooth box mapping and the quasi-box mapping. Let

$$
\mathcal{V}_{Q}=\bigcup_{c \in \operatorname{Crit}(f) \cap \omega\left(c_{0}\right)} \hat{V}_{c}
$$



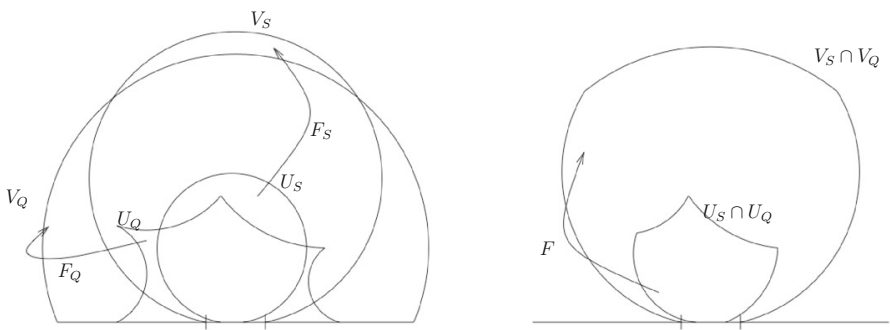

Fig. 17. Intersecting a smooth box mapping with a quasi-box mapping

where the $\hat{V}_{c}$ are as constructed at the beginning of Step 2. We construct a quasi-box mapping $F: \mathcal{U}_{Q} \rightarrow \mathcal{V}_{Q}$ extending the real return map to $I_{n}$ as follows. Since the hypotheses from the second part of Corollary 9.4 hold, we obtain a qr quasi-box mapping $\hat{F}_{Q}: \hat{\mathcal{U}}_{Q} \rightarrow \hat{V}_{c_{0}}$ extending the return map to $I_{n}$.

Now let $\mathcal{U}_{Q}=\hat{\mathcal{U}}_{Q}$. For each $U \in \mathcal{U}_{Q}$ choose $x \in U \cap \omega\left(c_{0}\right)$ and let $s>0$ be minimal so that $f^{s}(x) \in \mathcal{V}_{Q}$. Set $F_{Q}\left|U=f^{s}\right| U$.

We get a smooth box mapping $F_{S, n}: \mathcal{U}_{S, n} \rightarrow \mathcal{V}_{S, n}$ extending $R_{\hat{I}_{n}}$ as follows. There exists $k_{n}$ such that $\left.F_{S}^{k_{n}}\right|_{I_{n}}=\left.f^{p_{n-1}+p_{n-2}+\cdots+p_{n-m}}\right|_{I_{n}}$. Let $V_{S, n}\left(c_{0}\right)=$ $\operatorname{Comp}_{c_{0}} F_{S}^{-k_{n}}\left(A\left(c_{0}\right)\right)$. For each critical point $c \in \operatorname{Crit}(f) \cap \omega\left(c_{0}\right)$, let $i_{c}>0$ be minimal so that $F_{S}^{i_{c}}(c) \in I_{n}$. Let $V_{S, n}(c)=\operatorname{Comp}_{c}\left(F_{S}^{-i_{c}}\left(V_{S, n}\left(c_{0}\right)\right)\right)$ and

$$
\mathcal{V}_{S, n}=\bigcup_{c \in \operatorname{Crit}(f) \cap \omega\left(c_{0}\right)} V_{S, n}(c) \text {. }
$$

If $x \in \omega\left(c_{0}\right) \cap \mathcal{V}_{S, n}$, let $k \geq 1$ be minimal so that $F_{S}^{k}(x) \in \mathcal{V}_{S, n}$. Let $V=\operatorname{Comp}_{F_{S}^{k}(x)} \mathcal{V}_{S, n}$, and set $U_{S, n}(x)=\operatorname{Comp}_{x}\left(F_{S}^{-k}(V)\right)$.

Finally, for each critical point $c \in \omega\left(c_{0}\right)$, let $V_{c}=V_{S, n}(c) \cap \hat{V}_{c}$ and let $\mathcal{V}$ be the union of the domains $V_{c}$. For each $x \in \omega\left(c_{0}\right) \cap \mathcal{V}$, let $\hat{U}(x)$ be the component of $\hat{\mathcal{U}}_{Q}$ that contains $x$ and set $U(x)=U_{S, n}(x) \cap \hat{U}(x)$. Let $\mathcal{U}$ be the union of all domains $U(x)$. Define $F|U(x)=\hat{F}| U(x)$. See Fig. 17. Then $F: \mathcal{U} \rightarrow \mathcal{V}$ is a qr box mapping that extends the real return map to $I_{n}$.

Since for all $j$, with $1 \leq j \leq m+1$, the interval $I_{n-j}$ is $\delta$-free, there exists $\theta \in(0, \pi)$, depending on $\theta_{2}$ and $\delta$, so that for any component $V$ of $\mathcal{V}, V \supset D_{\theta}(V \cap \mathbb{R})$. Hence there exists $\eta>0$ such that $\operatorname{diam}(V)<\eta|V \cap \mathbb{R}|$.

Corollary 10.3. Suppose that $R_{\hat{I}_{0}}: D\left(\hat{I}_{0}\right) \rightarrow \hat{I}_{0}$ is non-renormalizable at $c_{0}$. Then for all $n$ sufficiently large, there exists a qr box mapping $F_{n}: \mathcal{U}_{n} \rightarrow \mathcal{V}_{n}$ extending $R_{\hat{I}_{n}}$.

Proof. Let $v_{1} \geq 1$ be the constant coming from Corollary 8.8. Let $M$ be the constant associated to $v_{1}$ by Proposition 10.2. There are two cases: either there exists $N \in \mathbb{N}$ so that $\left|I_{j}\right| /\left|I_{j+1}\right| \leq v_{1}$ for all $j>N$, or there exist infinitely many integers $j$ such that $\left|I_{j}\right| /\left|I_{j+1}\right|>v_{1}$. Using either Proposition 10.2, or Corollary 8.8, we construct $F: \mathcal{U} \rightarrow \mathcal{V}$ a qr box mapping extending $R_{\hat{I}_{n}}$, for an integer $n$ sufficiently large.

Let $k_{1} \in \mathbb{N}$ be such that $\left.F^{k_{1}}\right|_{I_{n+1}}=\left.f^{p_{n}}\right|_{I_{n+1}}$. Let $V\left(c_{0}\right)$ be the component of $\mathcal{V}$ that contains $c_{0}$, and define

$$
V_{1}\left(c_{0}\right)=\operatorname{Comp}_{I_{n+1}} F^{-k_{1}}\left(V\left(c_{0}\right)\right) \text {. }
$$


We can obtain a qr box mapping $F_{1}: \mathcal{U}_{1} \rightarrow \mathcal{V}_{1}$ that extends $R_{\hat{I}_{n+1}}: \mathrm{D}\left(\hat{I}_{n+1}\right) \rightarrow \hat{I}_{n+1}$ using, for example, the argument used in the proof of Step 3 in Proposition 10.2. Similarly, we can define a sequence of qr box mappings $F_{i}: \mathcal{U}_{i} \rightarrow \mathcal{V}_{i}$ each extending $R_{\hat{I}_{n+i}}$.

We will bound the quasiconformal distortion of the maps $F_{i}$ in the next subsection.

To prove complex bounds, as stated in Theorem 10.1, it suffices to prove certain geometric bounds known as upper and lower bounds in [KSvS], see p. 16. This is done in Sects. 10.2 and 10.3.

10.2. Upper bounds for finitely renormalizable maps. To prove Proposition 1.9, we follow the approach used in the proof of [KSvS, Proposition 8.3].

It will be convenient to point out the following modification to the proof in [KSvS] now. In $[\mathrm{KSvS}]$, an interval $I$ is said to be in $\mathcal{T}_{\xi}$ if $I$ is $\xi$-nice and $(1+2 \xi) I \backslash(1+2 \xi)^{-1} I$ is disjoint from the post-critical set of $f$. We modify this definition to take into account odd critical points: if $c_{0}$ is even, then we will say that an interval $I$ is in $\mathcal{T}_{\xi}$ if $I$ is $\xi$-nice and $\xi$-free; if $c_{0}$ is odd, then we say that $I$ is in $\mathcal{T}_{\xi}$ if $I$ is $\xi$-nice and $\xi$-externally free. It is worth remarking that this implies that $I$ is $\xi^{\prime}$-strongly nice, with $\xi^{\prime} \rightarrow \infty$ as $\xi \rightarrow \infty$.

Let $F: \mathcal{U} \rightarrow \mathcal{V}$ be a qr box mapping given by Proposition 10.2 or by Corollary 8.8 and let $V_{c_{0}}$ be the component of $\mathcal{V}$ that contains $c_{0}$. For ease of exposition, assume $F$ extends the return map to $\hat{I}_{0}=\cup_{c \in \operatorname{Crit}(f) \cap \omega\left(c_{0}\right)} \hat{\mathcal{L}}_{c}\left(V_{c_{0}} \cap \mathbb{R}\right)$, and let $\boldsymbol{I}_{0}=V_{c_{0}}$. Assuming that $\boldsymbol{I}_{n}$ is defined, let $\boldsymbol{I}_{n+1}=\operatorname{Comp}_{c_{0}} f^{-p_{n}}\left(\boldsymbol{I}_{n}\right)$, where $p_{n}$ is defined as on p. 22. Notice that for all $n \in \mathbb{N} \cup\{0\}, I_{n}=\boldsymbol{I}_{n} \cap \mathbb{R}$. We define the complex puzzle pieces $\mathcal{B}(\boldsymbol{I}), \mathcal{A}(\boldsymbol{I})$ and $\Gamma(\boldsymbol{I})$, associated to a domain $\boldsymbol{I}$, exactly as we did for real puzzle pieces.

We will need the following version of Proposition 7.1, which gives us information about the loss of angle even when the combinatorial depth $k(I, \mathbb{G})$ is large, see definition on p. 64.

Proposition 10.4 [KSvS, Proposition 11.2]. For each $\delta>0$, there exists $\mu \in(0,1)$ and $\delta^{\prime}>0$, and for each $\theta \in(0, \pi)$, there exists $\varepsilon>0$, such that the following holds. Let I be a $\delta$-nice interval with $|I|<\varepsilon$, and let $\mathbb{G}=\left\{G_{i}\right\}_{i=0}^{s}$ be a disjoint chain with $G_{0}, G_{s}$ nice intervals and $G_{0} \cap \omega\left(c_{0}\right) \neq \emptyset$. Let $\hat{G}_{s}$ be an interval with $G_{s} \subset \hat{G}_{s} \subset(1+2 \delta) \hat{G}_{s} \subset I$. Let $V=D_{\theta}\left(\hat{G}_{s}\right)$, and write $U_{i}=\operatorname{Comp}_{G_{i}} f^{-(s-i)}(V), i=0,1, \ldots, s$. Then there exists an inteval $\hat{I} \supset G_{0}$ with $\left(1+2 \delta^{\prime}\right) \hat{I} \subset I$ and such that

$$
U_{0} \subset D_{\mu^{k(I, \mathbb{G})} \theta}(\hat{I}) .
$$

Proof. The proof of this result proceeds by induction as in [KSvS]. To obtain the proof when $N=0$, we use the proof of the $N=0$ case of Proposition 7.1. If $c$ is even, which we can and will assume whenever $c_{0}$ is even, [KSvS, Lemma 11.2] holds by Lemma 3.12. When all critical points are odd, we do not need [KSvS, Lemma 11.2] to obtain that certain intervals are $\hat{\rho}$-nice, since we can use Proposition 6.2 instead of Corollary 4.8. The remainder of the proof of Proposition 11.2 in $[\mathrm{KSvS}]$ can be repeated verbatim, where we use Lemma 3.16 instead of [KSvS, Lemma 9.7], Theorem 3.2 (1) instead of [KSvS, Lemma 9.2], Lemma 3.6 instead of [KSvS, Lemma 9.4], Proposition 6.3 instead of [KSvS, Lemma 11.1], and Lemma 4.6 and its Corollaries, Corollary 4.7 and Corollary 4.8, instead of Lemmas 13.5 and 13.6 of [KSvS].

The following proposition is the main tool that is needed to combine the bounded and big geometry cases. Without control on the geometry, there is no way to control the 
combinatorial depth between consecutive levels of the enhanced nest, but we are still able to control the geometry at deeper levels, see the last part of the following proposition.

Proposition 10.5 [KSvS, Proposition 11.3]. For each $\delta>0$ and $N \geq 0$ there exist $\mu(N, \delta) \in(0,1)$ and $C \in(0,1)$, and for each $\theta \in(0, \pi)$ there exists $\varepsilon>0$ so that the following holds. Let $I \ni c_{0}$ be an interval in $\mathcal{T}_{\delta}$ with $|I|<\varepsilon$. Let $J \ni c_{0}$ be an (at most) $N$-modal pullback of $I$, and let $t$ be so that $J=\operatorname{Comp}_{c_{0}}\left(f^{-t}(I)\right) \cap \mathbb{R}$. Assume $x \in J \cap \omega\left(c_{0}\right)$ is so that $f^{s}(x) \in J$ for some $s \geq t$. Let

$$
v=\#\left\{0 \leq j \leq s-t: f^{j}(x) \in J\right\} .
$$

Let $s_{0}=0<s_{1}<\cdots<s_{v}$ be the times for which $s_{j} \leq s-t$ and $f^{s_{i}}(x) \in J$. Consider the chain $\mathbb{G}:=\left\{G_{i}\right\}_{i=0}^{s}$ defined by $G_{s}=J$, and $G_{i} \ni f^{i}(x)$. Let $U_{s}=D_{\theta}(I) \cap \mathbb{C}_{G_{s}}$ and $U_{i}=\operatorname{Comp}_{G_{i}} f^{-(s-i)}\left(U_{s}\right)$. Then,

$$
U_{0} \subset D_{\theta^{\prime}}(J),
$$

where

$$
\theta^{\prime}=\min \left[\mu^{\hat{k}(I, J)}\left(\Pi_{j=0}^{v-1} C \rho_{j}\right) \cdot \theta, \theta_{0}\right]
$$

and $\hat{k}(I, J)$ is defined on $p .64$ and $\rho_{j}>0$ is so that

$$
\left(1+2 \rho_{j}\right) \mathcal{L}_{f^{s}(x)}(J) \subset J
$$

and $\theta_{0}$ is defined in Lemma 4.5 .

Moreover, there exists a universal (large) constant $\xi>0$ and $\hat{v} \in \mathbb{N}$, which depends on $\delta$ and $N$, such that if $J$ is $\xi$-nice and $\xi$-externally free, $v \geq \hat{v}$ and for each $c \in$ $\operatorname{Crit}(f) \cap \omega\left(c_{0}\right)$, different form $c_{0}$

$$
\#\left\{0 \leq j \leq s: f^{j}(x) \in \mathcal{L}_{c}(J)\right\} \geq v_{0},
$$

then

$$
\theta^{\prime}=\min \left[\theta, \theta_{0}\right]
$$

Proof. The proof of this is the same as the proof of [KSvS, Proposition 11.3] using Proposition 10.4 instead of [KSvS, Proposition 11.2], Lemma 3.15 instead of [KSvS, Lemma 9.6], Lemma 4.5 instead of [KSvS, Lemma 13.4], and Lemmas 4.6 and 6.2 instead of Lemma 13.5.

The following result is the key initial estimate needed to prove the Upper Bounds:

Proposition 10.6 [KSvS, Theorem 11.1]. There exist $\theta \in(0, \pi)$ and $n_{0} \in \mathbb{N}$ so that for all $n$ sufficiently large, $\boldsymbol{I}_{n} \subset D_{\theta}\left(I_{n-n_{0}}\right)$.

Proof. We can repeat the proof Theorem 11.1 of $[\mathrm{KSvS}]$ after making the following substitutions: we use Lemma 4.5 instead of [KSvS, Lemma 13.4], Proposition 10.5 instead of [KSvS, Proposition 11.3], Theorem 3.1 instead of [KSvS, Proposition 8.1] Theorem 9.3 instead of [KSvS, Proposition 11.4], and Lemma 2.3 instead of [KSvS, Lemma 8.3]. 
Proposition 10.7 [KSvS, Proposition 11.5]. There exists $\theta \in(0, \pi)$ such that for all $n$ sufficiently large and for each $x \in \omega\left(c_{0}\right) \cap I_{n}$,

$$
\mathcal{L}_{x}\left(\boldsymbol{I}_{n}\right) \subset D_{\theta}\left(I_{n}\right)
$$

This proposition follows immediately from the following two lemmas whose proofs can be copied from $[\mathrm{KSvS}]$ making the same substitutions that we have already pointed out:

Lemma 10.8 [KSvS, Lemma 11.4]. There exist $N$ and $\theta_{1} \in(0, \pi)$ such that for each $n$ sufficiently large there exists $m \in\{n, n+3\}$ such that for each $x \in \omega\left(c_{0}\right) \cap I_{m}$ one has

$$
\operatorname{Comp}{ }_{x} \operatorname{Dom}\left(R_{I_{m}}^{N}\right) \subset D_{\theta_{1}}\left(I_{m}\right)
$$

Lemma 10.9 [KSvS, Lemma 11.5]. There exists a constant $\mu \in(0,1)$ such that for each $N \geq 2$, each $\theta \in(0, \pi)$, and each $n$ sufficiently large, if

$$
\cup_{x \in \omega\left(c_{0}\right) \cap I_{n}} \operatorname{Comp}_{x} \operatorname{Dom}\left(R_{\boldsymbol{I}_{n}}^{N}\right) \subset D_{\theta}\left(I_{n}\right),
$$

then

$$
\cup_{x \in \omega\left(c_{0}\right) \cap I_{n+1}} \operatorname{Comp}_{x} \operatorname{Dom}\left(R_{\boldsymbol{I}_{n+1}}^{N-1}\right) \subset D_{\mu \theta}\left(I_{n+1}\right) .
$$

Using this, we can bound the quasiconformal distortion as we pullback through the enhanced nest.

Lemma 10.10. There exists a constant $\eta_{1}>0$ such that the following holds. Let $\hat{I}_{0}$ be a nice neighbourhood of Crit $(f)$ such that $R_{\hat{I}_{0}}$ extends to a qr box mapping $F: \mathcal{U} \rightarrow \mathcal{V}$. Let $\boldsymbol{I}_{0}=\operatorname{Comp}_{c_{0}}(\mathcal{V})$. Assuming that $\boldsymbol{I}_{n}$ has been constructed let $\boldsymbol{I}_{n+1}=\operatorname{Comp}_{c_{0}} F^{-p_{n}}\left(\boldsymbol{I}_{n}\right)$, where $p_{n}$ is as in the construction of the enhanced nest (see p. 22). Let $s=p_{n}+p_{n-1}+$ $\cdots+p_{0}$. Then $F^{s}: \boldsymbol{I}_{n+1} \rightarrow \boldsymbol{I}_{0}$ is $1+\eta_{1} \mu\left(I_{0}\right)^{1 / 2}$-quasiregular.

Proof. By Lemma 5.10 and Propositions 10.7 and there exists a constant $\eta>0$ such that each $F^{p_{n}}: \boldsymbol{I}_{n+1} \rightarrow \boldsymbol{I}_{n}$ is $\left(1+\eta \mu\left(I_{n}\right)^{1 / 2}\right)$-quasiregular. Since, by Theorem 3.1 , there exists a universal constant $\rho>0$ such that each $I_{n}$ is $\rho$-nice, the lengths of the intervals $I_{n}$ decays exponentially. Thus there exists a constant $\eta_{1} \geq 0$ such that $F^{s}: I_{n+1} \rightarrow \boldsymbol{I}_{0}$ is $1+C \eta_{1} \mu\left(I_{0}\right)^{1 / 2}$-quasiregular.

Finally with Proposition 10.7 in hand we can repeat the proof of the Upper Bounds, Proposition 8.3 from [KSvS].

Proposition 10.11 (Upper bounds for the enhanced nest). There exists a constant $\eta>0$ such that for all $n$ sufficiently large the following hold.

- $\operatorname{diam}\left(\boldsymbol{I}_{n}\right) \leq \eta\left|I_{n}\right|$, and;

- there exists a topological disk $\Omega \supset \boldsymbol{I}_{n}$ such that $\left(\Omega \backslash \boldsymbol{I}_{n}\right) \cap \omega\left(c_{0}\right)=\emptyset$ and

$$
\bmod \left(\Omega \backslash \boldsymbol{I}_{n}\right)>1 / \eta
$$


Proof. By construction there exists a positive integer $v$ and by Proposition 10.7, there exists $\theta^{\prime} \in(0, \pi)$ such that $f^{v}: \mathcal{B}\left(\boldsymbol{I}_{n}\right) \rightarrow \boldsymbol{I}_{n}$ is a proper map with bounded degree, $f^{v}\left(\mathcal{A}\left(\boldsymbol{I}_{n}\right)\right)=\mathcal{L}_{f^{v}(c)}\left(\boldsymbol{I}_{n}\right)$, and $\mathcal{L}_{f^{v}(c)}\left(\boldsymbol{I}_{n}\right) \subset D_{\theta^{\prime}}\left(I_{n}\right)$.

Then, since by Theorem 3.1, there exists $\rho>0$ so that $I_{n}$ is $\rho$-nice, $\rho$-externally free, the pullback of $I_{n}$ to $\mathcal{B}\left(I_{n}\right)$ is of bounded degree, and we have that either $c_{0}$ is of even order and $I_{n}$ is $\rho$-free or $c_{0}$ is of odd order, there exists $\lambda \in(0,1)$ such that

$$
\mathcal{A}\left(\boldsymbol{I}_{n}\right) \subset D_{\lambda \theta}\left(\mathcal{B}\left(I_{n}\right)\right),
$$

and

$$
\frac{\operatorname{diam}\left(\mathcal{A}\left(\boldsymbol{I}_{n}\right)\right)}{\left|\mathcal{A}\left(I_{n}\right)\right|} \leq \eta(\theta) \max \left\{1,\left(\frac{\operatorname{diam}\left(\boldsymbol{I}_{n}\right)}{\left|I_{n}\right|}\right)^{1 / 2}\right\} .
$$

To prove this estimate, we let $r>0$ be the return time of $\mathcal{L}_{f^{v}\left(c_{0}\right)}\left(\boldsymbol{I}_{n}\right)$ to $\boldsymbol{I}_{n}$, and decompose $f^{v+r}: \mathcal{A}\left(\boldsymbol{I}_{n}\right) \rightarrow \boldsymbol{I}_{n}$ into $f^{\nu+r-1}: f\left(\mathcal{A}\left(\boldsymbol{I}_{n}\right)\right) \rightarrow \boldsymbol{I}_{n}$ and $f: \mathcal{A}\left(\boldsymbol{I}_{n}\right) \rightarrow f\left(\mathcal{A}\left(\boldsymbol{I}_{n}\right)\right)$, apply Lemma 5.10, and use the fact that there is a critical point $c_{0} \in \mathcal{A}\left(\boldsymbol{I}_{n}\right) \subset$ $\boldsymbol{I}_{n}$ of degree $d \geq 2$. Since $\mathcal{B}\left(I_{n}\right)$ is $\rho^{\prime}$-nice and $\mathcal{A}\left(I_{n}\right)$ is $\rho^{\prime}$-externally free for some $\rho^{\prime}>0$ given by Corollaries 3.4 and 3.5, it follows that there exist $\xi>0$, $\theta^{\prime} \in(0, \pi / 2)$ and a topological disk $\mathcal{A}\left(\boldsymbol{I}_{n}\right)^{\prime}, \mathcal{A}\left(\boldsymbol{I}_{n}\right) \Subset \mathcal{A}\left(\boldsymbol{I}_{n}\right)^{\prime} \subset D_{\theta^{\prime}}\left(\mathcal{B}\left(I_{n}\right)\right)$, so that $\bmod \left(\mathcal{A}\left(\boldsymbol{I}_{n}\right)^{\prime} \backslash \mathcal{A}\left(\boldsymbol{I}_{n}\right)\right)>\xi$ and $\left(\mathcal{A}\left(\boldsymbol{I}_{n}\right)^{\prime} \backslash \mathcal{A}\left(\boldsymbol{I}_{n}\right)\right) \cap \omega\left(c_{0}\right)=\emptyset$. Let $\tilde{v} \in \mathbb{N}$ be so that $f^{\tilde{v}}: \mathcal{B A}\left(\boldsymbol{I}_{n}\right) \rightarrow \mathcal{A}\left(\boldsymbol{I}_{n}\right)$. Let $F_{n}=\operatorname{Comp}_{c_{0}} f^{-\tilde{v}}\left(\mathcal{B}\left(I_{n}\right)\right)$. Then $f^{\tilde{v}} \mid F_{n}$ is a proper map of bounded degree, and either $F_{n}$ is $\delta^{\prime}$-free for some $\delta^{\prime}>0$ or $c_{0}$ is an odd critical point. Likewise, for each $i, i=1,2, \ldots, T$, let $t_{i} \in \mathbb{N}$, be so that $f^{t_{i}}: \Gamma^{i} \mathcal{B} \mathcal{A}\left(\boldsymbol{I}_{n}\right) \rightarrow \Gamma^{i-1} \mathcal{B} \mathcal{A}\left(\boldsymbol{I}_{n}\right)$. Inductively construct a sequence of domains $F_{n}^{i}$, $i=0, \ldots, T$, setting $F_{n}^{0}=F_{n}$ and $F_{n}^{i}=\operatorname{Comp}_{c_{0}} f^{-t_{i}}\left(F_{n}^{i-1}\right)$. Each $F_{n}^{i}$ is a pullback of $F_{n}^{i-1}$ of bounded degree and either $F_{n}^{i}$ is $\delta^{\prime \prime}$-free for some $\delta^{\prime \prime}>0$ or $c_{0}$ is an odd critical point. Set $\boldsymbol{I}_{n+1}^{\prime}=\operatorname{Comp}_{c_{0}}\left(f^{-\left(\tilde{v}+t_{1}+\cdots t_{T}\right.}\left(\mathcal{A}^{\prime}\left(\boldsymbol{I}_{n}\right)\right)\right)$. Observe that $\left(\mathbf{I}_{n+1}^{\prime} \backslash \mathbf{I}_{n+1}\right) \cap \omega\left(c_{0}\right)=\emptyset$.

By Lemma 5.10, we have that $f^{\tilde{v}+t_{1}+\cdots+t_{T}} \mid \boldsymbol{I}_{n+1}^{\prime}$ is a $1+c \mu\left(I_{n-1}\right)^{1 / 2}$-quasiregular mapping with bounded degree. It follows that there exists $c^{\prime} \geq 1$ so that $\bmod \left(\mathbf{I}_{n+1}^{\prime} \backslash \mathbf{I}_{n+1}\right)>$ $\xi / c^{\prime}$. As before,

$$
\frac{\operatorname{diam}\left(\boldsymbol{I}_{n+1}\right)}{\left|I_{n+1}\right|} \leq \eta(\theta) \max \left\{1,\left(\frac{\operatorname{diam}\left(\boldsymbol{I}_{n}\right)}{\left|I_{n}\right|}\right)^{1 / 2}\right\} .
$$

Since $\operatorname{diam}\left(\mathbf{I}_{0}\right)<\eta\left|I_{0}\right|$, it follows that $\operatorname{diam}\left(\boldsymbol{I}_{n}\right) /\left|I_{n}\right|$ is bounded from above.

It is worth noticing that from Propositions 1.9, 10.7 and the definitions of the operators $\mathcal{A}$ and $\mathcal{B}$ we have:

Corollary 10.12. There exists $\varepsilon>0$ and a universal constant $\delta>0$ such that, for all $n>0$ for which $\left|I_{n}\right|<\varepsilon$ the following holds. The puzzle piece $\boldsymbol{I}_{n}$ is $\delta$-nice and $\delta$-free. Hence the return mapping to $\boldsymbol{I}_{n}$ is $\delta$-extendible. Even more, there exists an interval $\tilde{I}_{n} \supset(1+2 \delta) I_{n} \tilde{I}_{n} \supset(1+2 \delta) I_{n}$ with $\left(\tilde{I}_{n} \backslash I_{n}\right) \cap \omega\left(c_{0}\right)$ so that $D_{\theta^{\prime}}\left(\tilde{I}_{n}\right) \supset \boldsymbol{I}_{n}$.

10.3. Lower bounds for finitely renormalizable maps. Lower Bounds for the geometry of puzzle pieces in the enhanced nest follows from the next two lemmas. The first lemma is Lemma 10.1 of [KSvS]; however, we cannot repeat the proof given there, since $f$ does not have to be analytic. 
Let

$$
\eta_{n}=\inf _{x \in \omega\left(c_{0}\right) \cap I_{n}} \frac{d\left(x, \partial \boldsymbol{I}_{n}\right)}{\left|I_{n}\right|} .
$$

Lemma 10.13 [KSvS, Lemma 10.1]. There exists $\varepsilon>0$ so that for all $n>0$ for which $\left|I_{n}\right|<\varepsilon$ the following holds:

1. There exists a constant $\delta>0$ such that $\eta_{n+1}>\delta \eta_{n}$.

2. There exist $\kappa>0, v>1$ such that if $\left|I_{n}\right| /\left|I_{n+1}\right|>v$, then

$$
\eta_{n+2} \geq \min \left(\kappa, 2 \eta_{n+1}\right) \text {. }
$$

Proof. Note that we can decompose the map $f^{p_{n}}: I_{n+1} \rightarrow I_{n}$ into a bounded number of maps, each of the form $z^{d}$ followed by a diffeomorphism with bounded distortion.

To prove the first estimate, observe that $\eta_{n+1} \geq \delta \eta_{n}$ is equivalent to

$$
\frac{\inf _{x \in \omega\left(c_{0}\right) \cap I_{n}} d\left(x, \partial \boldsymbol{I}_{n}\right)}{\inf _{x \in \omega\left(c_{0}\right) \cap I_{n+1}} d\left(x, \partial \boldsymbol{I}_{n+1}\right)} \leq \frac{1}{\delta} \frac{\left|I_{n}\right|}{\left|I_{n+1}\right|} .
$$

For any $x \in \omega\left(c_{0}\right) \cap I_{n}$, since $f^{p_{n}}$ has bounded degree and, by Theorem 3.1, $I_{n}$ has external free space, it follows from Theorem 3.2 that there exists a constant $c^{\prime}>1$ such that

$$
\left|\left(f^{p_{n}}\right)^{\prime}(x)\right| \leq c^{\prime} \frac{\left|I_{n}\right|}{\left|I_{n+1}\right|} .
$$

By Lemma 5.10, there exists $\eta>0$, such that $f^{p_{n}} \mid \boldsymbol{I}_{n+1}$ is $\left(1+\eta\left|\mu\left(I_{n}\right)\right|^{1 / 2}\right)$ quasiregular. So by the Stoilow Factorization Theorem we can express $f^{p_{n}} \mid \boldsymbol{I}_{n+1}=h \circ g$, where $g: \boldsymbol{I}_{n+1} \rightarrow \boldsymbol{I}_{n+1}$ is $\left(1+\eta\left|\mu\left(I_{n}\right)\right|^{1 / 2}\right)$-quasiconformal and $h: \boldsymbol{I}_{n+1} \rightarrow \boldsymbol{I}_{n}$ is holomorphic. Let $\lambda_{n}$ be an affine mapping that scales $\boldsymbol{I}_{n}$ to unit size. Then the mapping $H(z)=\lambda_{n} f^{p_{n}}\left(\frac{z}{\lambda_{n+1}}\right)$ is a $\left(1+\eta\left|\mu\left(I_{n}\right)\right|^{1 / 2}\right)$-quasiregular mapping between domains of unit size. As $n \rightarrow \infty$ this mapping converges to a holomorphic mapping. Arguing by contradiction, it follows that there exist constants $c>1$ and $\kappa_{1}>0$ such that

$$
\left|\left(f^{p_{n}}\right)^{\prime}(z)\right| \leq c \frac{\left|I_{n}\right|}{\left|I_{n+1}\right|}
$$

for $z \in \mathbb{C}$ with $\operatorname{dist}\left(z, \omega\left(c_{0}\right) \cap I_{n+1}\right) \leq \kappa_{1}\left|I_{n+1}\right|$, and the first estimate follows immediately.

Similarly, from the Koebe Distortion Theorem and the fact that $f^{p_{n}} \mid \boldsymbol{I}_{n+1}$ is $\left(1+\eta\left|\mu\left(I_{n}\right)\right|^{1 / 2}\right)$-quasiregular, we have that there exists $\kappa_{2}>0$ such that for any $z \in B\left(c_{0}, 2 \kappa_{2}\left|I_{n+1}\right|\right)$,

$$
\left|\left(f^{p_{n}}\right)^{\prime}(z)\right| \leq \frac{1}{2} \frac{\left|I_{n}\right|}{\left|I_{n+1}\right|} .
$$

Let $v>1$ be large. Notice that when $\left|I_{n}\right| /\left|I_{n+1}\right|>v$, then by Lemma 3.23 there exists $v_{1}>0$, so that for any $x \in \omega\left(c_{0}\right) \cap I_{n}$ we have $\left|I_{n+1}\right| /\left|\mathcal{L}_{x}\left(I_{n+1}\right)\right|>v_{1}$. From the definitions of the operators $\mathcal{A}, \mathcal{B}$ and Theorem $3.2(2)$, there exists a constant $\eta=\eta(\varepsilon) \rightarrow$ 0 as $\varepsilon \rightarrow 0$, so that $\omega\left(c_{0}\right) \cap \mathcal{B} \mathcal{A}\left(I_{n+1}\right) \subset \mathcal{A} \mathcal{A}\left(I_{n+1}\right)$ is contained in a $\eta\left|\mathcal{B} \mathcal{A}\left(I_{n+1}\right)\right|-$ neighbourhood of the critical point, and the second estimate in the lemma follows. 
The following is Lemma 10.2 of [KSvS]. The proof differs from that given in [KSvS] because $f$ is not analytic and we have to treat the big geometry case separately from the bounded geometry case.

Lemma 10.14 [KSvS, Lemma 10.2]. There exists $k_{0} \in \mathbb{N}$ and a constants $\gamma>0$ and $\varepsilon>0$ such that for all $n>0$ with $\left|I_{n}\right|<\varepsilon$ and for all $x \in \omega\left(c_{0}\right) \cap I_{n}$,

$$
B\left(x, \gamma\left|\operatorname{Comp}_{x} \operatorname{Dom}\left(R_{I_{n}}^{k_{0}}\right)\right|\right) \subset \boldsymbol{I}_{n} .
$$

Proof. Let $N=p_{n-1}+p_{n-2}+\cdots+p_{0}$. Observe that $f^{N}\left(\boldsymbol{I}_{n}\right)=\boldsymbol{I}_{0}$. By Lemma 8.3 of [KSvS], we have that $2 p_{n-1} \geq N$ and $r\left(I_{n}\right) \geq N / 6$. Let $x \in \omega\left(c_{0}\right) \cap I_{n}$ and let $W=\hat{\mathcal{L}}_{f^{N}(x)}\left(\boldsymbol{I}_{n}\right)$. Let $U=\operatorname{Comp}_{x}\left(f^{-N}(W)\right) \cap \mathbb{R}$. Then $U=\operatorname{Comp}_{x}\left(\operatorname{Dom}\left(R_{I_{n}}^{k_{0}}\right)\right)$ for some $k_{0}$, and since $r\left(I_{n}\right) \geq N / 6$ we have that $k_{0} \leq 6$ and that the order of $f^{N}: U \rightarrow W$ is bounded by $6 b$.

If either $c_{0}$ is even or $\left|I_{n-3}\right| /\left|I_{n-2}\right|$ is bounded from above, then by Theorem 3.1 $I_{n}$ is $\rho$-free for some $\rho>0$, and we can repeat the proof of Lemma 10.2 of [KSvS]. So, suppose that $\left|I_{n-3}\right| /\left|I_{n-2}\right|$ is big and $c_{0}$ is of odd order. In this case, $I_{n}$ is $C$-nice and $C$-externally free with $C$ big. Let $0<C^{\prime}<C$ be so that $\tilde{I}:=\left(1+2 C^{\prime}\right) I_{n}$ is $\delta$-externally free. Let $\tilde{W}=\hat{\mathcal{L}}_{f^{N}(x)}(\tilde{I})$, and $\tilde{U}=\operatorname{Comp}_{x}\left(f^{-N}((1+2 \delta) W)\right) \cap \mathbb{R}$. Since $C$ is big, we can choose $C^{\prime}$ so that $\delta>0$ does not depend on $f$ and so that $\tilde{W} \supset(1+2 \delta) W$. Then $\tilde{W}$ is $\rho^{\prime \prime}$-free for some $\rho^{\prime \prime}>0$. By Proposition 10.2 , there exists $\sigma \in(0, \pi / 2)$ so that $D_{\pi-\sigma}(\tilde{W}) \subset D_{\pi-\sigma}\left(I_{0}\right) \subset \boldsymbol{I}_{0}$. So Lemmas 5.10, 4.2 and 4.3, imply that $\boldsymbol{I}_{n} \supset \operatorname{Comp}_{x} f^{-N}\left(D_{\pi-\sigma}(\tilde{W})\right) \supset D_{\theta^{\prime \prime}}(\tilde{U})$, where $\theta^{\prime \prime} \in(0, \pi / 2)$.

Proposition 10.15. There exist beau constants $\eta>0$ and $\varepsilon>0$ such that for all $n>0$ with $\left|I_{n}\right|<\varepsilon$

$$
B\left(c_{0}, \eta\left|I_{n}\right|\right) \subset \boldsymbol{I}_{n}
$$

Proof. The proof follows the proof of Proposition 10.1 of [KSvS] with some minor adjustments to deal with the case when $c_{0}$ is odd. Indeed, there exists a constant $\varepsilon^{\prime}>0$ such that if $0<\eta_{n}<\varepsilon^{\prime}$, then $\left|I_{n+1}\right| /\left|I_{n}\right|<\varepsilon$, where $\varepsilon$ is the constant from the second estimate in Lemma 10.13. To see this, observe that if $\eta_{n}$ is very small, then by Lemma 10.14 there exists $x \in \omega\left(c_{0}\right) \cap I_{n}$ such that $|U| /\left|I_{n}\right|$ is very small where $U \ni x$ is a component of the domain of $R_{I_{n}}^{k_{0}}$. Since $k_{0}$ is bounded ( $\leq 6$, see the proof of Lemma 10.14), this implies that there exists a return domain $J$ to $I_{n}, J \cap \omega\left(c_{0}\right) \neq \varnothing$, and such that $|J| /\left|I_{n}\right|$ is very small. It follows from Theorem 3.1 (e) that $\left|I_{n+1}\right|$ is very small compared to $\left|I_{n}\right|$. Thus it follows from the second statement of Lemma 10.13 that when $\eta_{n}<\varepsilon^{\prime}, \eta_{n+2} \geq \min \left\{\kappa, 2 \eta_{n+1}\right\}$. By the first statement of Lemma 10.13, we have $\eta_{n+2} \geq \min \left(\kappa^{\prime}, 2 \eta_{n+1}\right)$ for all $n$, where $\kappa^{\prime}=\min \left(\delta^{2} \varepsilon^{\prime}, \kappa\right)$. Since $\eta_{0}, \eta_{1}$ are bounded away from 0 the result follows.

Thus we have concluded the proof of complex bounds in the non-renormalizable case, i.e. Theorem 1.1 (in the non-renormalizable case) and Theorem 10.1. Let us now turn to the infinitely renormalizable case.

10.4. Infinitely renormalizable maps. To construct a qr polynomial-like extension from a qr quasi-box mapping for infinitely renormalizable maps, we use a more geometric approach. Let $L: \mathbb{R}_{+} \rightarrow \mathbb{R}_{+}$. A map $h: X \rightarrow Y$ between two metric spaces is called 
an $L$-quasi-isometry if for any $\varepsilon>0$, $\operatorname{dist}(h(x), h(y)) \leq \max \{L(\varepsilon) \operatorname{dist}(x, y), \varepsilon\}$, for all $x, y \in X$. Quasiconformal maps are quasi-isometries with respect to the hyperbolic metric:

Lemma 10.16 (Lemma 2.2, [ALdM]). For every $\kappa \geq 1$ there exists $L_{\kappa}: \mathbb{R}_{+} \rightarrow \mathbb{R}_{+}$such that if $h: S \rightarrow \tilde{S}$ is a $\kappa$-qc map between two hyperbolic Riemann surfaces, then $h$ is a $L_{\kappa}$ quasi-isometry in the hyperbolic metric. Furthermore, for every $\varepsilon>0, \lim _{\kappa \rightarrow 1} L_{\kappa}(\varepsilon)=$ 1.

Lemma 10.17 (cf. [LY], Lemma 2.4, [M2] Proposition 4.10). For every $\varepsilon, \eta>0$, there exists $\kappa_{0}>1$ such that if $1 \leq \kappa \leq \kappa_{0}$, there exists $\delta>0$ such that the following holds. Let $U \subset V$ be two real-symmetric topological disks and $f: U \rightarrow V$ be a realsymmetric qr branched covering with non-escaping critical points and compact Julia set. Suppose that $U$ contains an $\eta$-neighbourhood, $\mathcal{N}$ of the $J(f)$ in the hyperbolic metric on $U$, and that $f \mid \mathcal{N}$ is $\kappa$-quasiregular. Then there are real-symmetric topological disks $U \supset V^{\prime} \supset U^{\prime} \supset K(f)$ such that the restriction $f: U^{\prime} \rightarrow V^{\prime}$ is polynomial-like. Moreover, if $\bmod (U \backslash K(f)) \geq \varepsilon>0$, then $\bmod \left(V^{\prime} \backslash U^{\prime}\right) \geq \delta(\varepsilon, \ell, \eta)>0$, where $\ell$ is the degree of $f$.

Proof. The proof of this lemma goes as the proof of Proposition 4.10 of [M2], we will provide a sketch of the argument. Notice that since $f$ is a real-symmetric mapping between real-symmetric domains $U$ and $V, K(f)$ and the $\eta$-neighbourhood of $K(f)$ in the hyperbolic metric on $U$ are both real-symmetric. Observe that we can assume that $\bmod (V \backslash K(f))$ is bounded from above. This implies that $\mathcal{N} \backslash K(f)$ is an annulus with modulus bounded from below, so that its core curve $\gamma$ is a $\kappa^{\prime}$-quasicircle. Let $\mathcal{N}_{\gamma}$ denote the region bounded by $\gamma$. Then, by the Schwarz Reflection Principle, $f \mid \mathcal{N}_{\gamma}$ extends to a $\kappa^{\prime \prime}$-quasiregular mapping $\tilde{f}: U \rightarrow V$ that agrees with $f$ on $\mathcal{N}_{\gamma}$. Consider the hyperbolic Riemann surfaces $\mathcal{N}_{0} \subset U_{0} \subset V_{0}$ obtained by doubling $\mathcal{N}_{\gamma} \backslash K(f), U \backslash K(f)$ and $V \backslash K(f)$, respectively, across their ends corresponding to $K(f)$. Then $\mathcal{N}_{0} \subset U_{0} \subset V_{0}$ are annuli with the same core geodesic $\gamma$, and $\tilde{f} \mid U \backslash K(f)$ extends to a symmetric mapping $F: U_{0} \rightarrow V_{0}$ that sends $\gamma$ to itself.

Let us show that $\|D F(z)\|>1$ (in the hyperbolic metric) for $z$ sufficiently close to $\gamma$. Take $\tau>0$ and $z \in \gamma$, consider the balls $B_{U_{0}}(z, \tau), B_{V_{0}}(z, \tau)$ in terms of the hyperbolic metrics on $U_{0}$ and $V_{0}$, respectively. Provided we take $\tau>0$ sufficiently small, we get that the distance of the ball $B_{U_{0}}(z, \tau)$ to $V_{0} \backslash U_{0}$ is strictly positive. Then $F\left(B_{U_{0}}(z, \tau)\right) \supset B_{U_{0}}(F(z), \tau)$ provided $\kappa$ and $\tau$ are sufficiently small. Indeed, $F \mid \mathcal{N}_{0}$ is a composition of a $\kappa$-qc map and a conformal isometry from the hyperbolic metric on $U_{0}$ to the hyperbolic metric on $V_{0}$. Therefore $F\left(B_{U_{0}}(z, \tau)\right) \supset B_{V_{0}}(F(z), r \tau)$ where $r<1$ can be chosen arbitrarily close to one provided $\kappa$ is sufficiently close to one. Moreover, the contraction of the inclusion $U_{0} \rightarrow V_{0}$ on $B_{U_{0}}(z, \tau)$ is bounded from above by a constant $t<1$ since the distance of the ball $B_{U_{0}}(z, \tau)$ to $V_{0} \backslash U_{0}$ is strictly positive. It follows that $F\left(B_{U_{0}}(z, \tau)\right) \supset B_{U_{0}}(F(z), \kappa \tau)$ for some $\kappa>1$.

Let $V_{0}^{\prime} \subset V_{0}$ be the $\tau$-collar neighbourhood of $\gamma$, and let $U_{0}^{\prime}=F^{-1}\left(V_{0}^{\prime}\right)$. Set $U^{\prime}=\left(U_{0}^{\prime} \cap U\right) \cup K(f)$ and $V^{\prime}=f\left(U^{\prime}\right)$. Then $f: U^{\prime} \rightarrow V^{\prime}$ is a polynomial-like map and $\bmod \left(V^{\prime} \backslash U^{\prime}\right)$ is bounded away from zero.

Theorem 10.18 (Complex bounds in the infinitely renormalizable case). There exist $\delta>0$ and $C>0$ such that the following holds. Suppose that $f: M \rightarrow M$ is infinitely renormalizable at $c_{0}$, with periodic intervals $M \supset J_{1} \supset J_{2} \supset \cdots$ with periods $1<j_{1}<$ $j_{2}<\cdots$. Suppose that $i$ is sufficiently large. Then there exists a $\kappa\left(V_{i}\right)$-qr polynomial-like 
mapping $F_{i}: U_{i} \rightarrow V_{i}$ which extends $f^{j_{i}}: J_{i} \rightarrow J_{i}$ such that $U_{i}, V_{i}$ are real-symmetric, $\bmod \left(V_{i} \backslash U_{i}\right)>\delta, U_{i}$ has $\delta$-bounded geometry and $\operatorname{diam}\left(U_{i}\right)<C\left|J_{i}\right|$.

Proof. Let $I_{n}$ be the smallest puzzle piece in the generalized enhanced nest such that $I_{n} \supset J_{i}$. Then $I_{n}$ is a terminating interval. Note that $J_{i}=I_{n}$ if and only if $I_{n-1}$ is terminating and $J_{i}=\mathcal{R}\left(I_{n-1}\right)$.

Step 1: Moduli bounds. We will show that if $i$ is sufficiently big, the return mapping to $J_{i}$ extends to a quasi-box mapping $F_{Q}: U_{Q} \rightarrow V_{Q}$, and that $\bmod \left(V_{Q} \backslash K\left(F_{Q}\right)\right)$ is universally bounded from below.

We will show that $\bmod \left(V_{Q} \backslash K\left(F_{Q}\right)\right)$ is bounded from below by proving that the filled Julia set of the quasi-box mapping, $K\left(F_{Q}\right)$, is contained in a Poincaré disk, $D_{\theta}(K)$, where $K$ is an interval well-inside $U_{Q} \cap \mathbb{R}$, with $|K|$ comparable to $\left|I_{n}^{\infty}\right|$.

We will divide the proof in two cases, depending on the geometry of the generalized enhanced nest. To do so, we define a constant $v>0$ that will separate the bounded geometry case from the big geometry one.

Claim. There exists $v>0$ so that the following holds:

(a) If $\left|I_{n}\right| /\left|I_{n}^{\infty}\right|>v$, then the first return mapping to $I_{n}^{\infty}$ extends to a polynomiallike map $F: U \rightarrow V$ with $V=D_{\pi / 2}\left(I_{n}^{j}\right)$ with $\left|I_{n}^{j}\right| \asymp\left|I_{n}^{\infty}\right|$, for some $j \in \mathbb{N}$. Furthermore, there exists an interval $K$ well-inside $I_{n}^{j+1}$, with $|K| \asymp\left|I_{n}^{\infty}\right|$, so that $K(F) \subset D_{\pi / 2}(K)$.

(b) If $I_{n-1}$ is non-terminating and $\left|I_{n-1}\right| /\left|I_{n}\right|>v$ then the first return mapping to $I_{n}$ extends to a qr box mapping $F: U \rightarrow V$ with $V=D_{\pi / 2}\left(I_{n}^{j}\right)$ for some $j \in \mathbb{N}$. Furthermore, there exists an interval $K$ well-inside $I_{n}^{j+1}$, with $|K| \asymp\left|I_{n}^{\infty}\right|$ so that $K(F) \subset D_{\pi / 2}(K)$.

(c) If $I_{n-1}$ is terminating and $\left|\mathcal{R}\left(I_{n-1}\right)\right| /\left|I_{n}\right|>v$, then the first return mapping to $I_{n}^{\infty}$ extends to a qr box mapping with range $D_{\pi / 2}\left(I_{n}^{j}\right)$ for some $j \in \mathbb{N}$. Furthermore, there exists an interval $K$ well-inside $I_{n}^{j+1}$, with $|K| \asymp\left|I_{n}^{\infty}\right|$ so that $K(F) \subset D_{\pi / 2}(K)$.

(d) Assume $I_{n-1}$ non-terminating and $\left|I_{n-1}\right| /\left|I_{n}\right|>v$. Then the return map to $I_{n-1}^{\infty}$ extends to a polynomial like map $F: U \rightarrow V$ with $V \cap \mathbb{R}=I_{n-1}^{j}$, for some $j \in \mathbb{N}$, with the property that $\left|I_{n-1}^{j}\right| \asymp\left|I_{n-1}^{\infty}\right|$.

Proof of Claim. We will prove (a), the other cases follow using similar arguments. By the proof of Lemma 8.9, we know that $I_{n}^{2}$ is $C$-nice and $C$-free for some $C>0$, with $C \rightarrow \infty$ as $v \rightarrow \infty$. The Koebe Principle, Theorem 3.2 and Lemma 3.10 imply that there exists $j \in \mathbb{N}$ so that $\left|I_{n}^{j}\right| /\left|I_{n}^{j+1}\right|>v_{1}$, where $\nu_{1}>0$ is defined in Lemma 8.9, with $\left|I_{n}^{j}\right|$ is comparable to $\left|I_{n}^{\infty}\right|$. The claim follows using a similar argument as the one used in Lemma 8.9, except for the fact that we pull back two times under the return map to $I_{n}^{\infty}$, instead of one.

Cases (a), (b) and (c) reduce the proof to the following: either,

- $\quad I_{n-1}$ is terminating and $\left|I_{n}^{\infty}\right|$ is comparable to $\left|\mathcal{R}\left(I_{n-1}\right)\right|$, or

- $\quad I_{n-1}$ is non-terminating and $\left|I_{n-1}\right|$ is comparable to $\left|I_{n}^{\infty}\right|$.

Let $M \in \mathbb{N}$ be the constant associated to $v$ given by Theorem 9.3.

Case A: assume $J_{i} \subsetneq I_{n}$. 
Case A.1: Bounded geometry. Suppose that for all $0 \leq j \leq M,\left|I_{n-j}\right| /\left|I_{n-j+1}\right| \leq v$. Then $\left|I_{n+1}\right| \asymp\left|I_{n-M}\right|$. Let $F_{Q}: U_{Q} \rightarrow V_{Q}$ be the qr quasi-box mapping associated to the return mapping to $I_{n}$ given by Theorem 9.3, where $V_{Q}=D_{\pi / 2}\left(I_{n-M}\right) \cap \mathbb{C}_{I_{n}}$ and $U_{Q} \subset D_{\pi / 2}\left(I_{n-M+1}\right)$.

Now, we will show the moduli bounds between $V_{Q}$ and $K\left(F_{Q}\right)$ hold. We will choose a constant $k_{0} \in \mathbb{N}$ in the course of the proof. Let $\mathbb{G}=\left\{G_{l}\right\}_{l=0}^{k_{0} j_{i}}$ with $G_{k_{0} j_{i}}=J_{i}$ and $G_{0}=J_{i}$. To begin, we take $k_{0}$ at least 4(4) +1 . Let us recall that in Proposition 9.1, given a point $x \in I_{n} \cap \omega\left(c_{0}\right)$ with return time to $I_{n}$ equal to $s$ we constructed a sequence of times $0 \leq s_{n-4}<s_{n-5}<\cdots<s_{n-M}=s$ for which the pullbacks of certain Poincare disks are well controlled. In cases (a), (b) and (c) of the proof of Proposition 9.1, assuming that $s_{n-i-1}$ has been defined for $0 \leq i \leq M-1$, we can define $s_{n-i}$ provided that there are at least four intervals $G_{l} \subset I_{n-i}$ with $0 \leq l<s_{n-i-1}$. In case (d), to be able to define $s_{n-i}$ we need at least three of the intervals $G_{l}$ with $0 \leq l<s_{n-i-1}$ to be contained in $I_{n-i+1}$. Now, we apply the argument form Proposition 9.1 to the chain $\mathbb{G}$. Observe that, since $k_{0}-1 \geq 16$, we can repeat Step 2 of Proposition 9.1, to carry on the construction of the sequence $s_{i}$, to obtain $s_{n-1}<s_{n-2}<s_{n-3}<s_{n-4}<\cdots<s_{n-M}=k_{0} j_{i}$. Then, using Lemma 9.2, as in the proof of Theorem 9.3, we find $\theta_{1} \in(0, \pi)$, so that

$$
\operatorname{Comp}_{c_{0}} f^{-\left(k_{0} j_{i}-s_{n-1}\right)}\left(V_{Q}\right) \subset D_{\theta_{1}}\left(I_{n-1}\right) .
$$

Suppose that $I_{n-1}$ is non-terminating. Using Proposition 8.2 and the choice of $k_{0}$ we define $0 \leq s_{n}<s_{n-1}$, and obtain an interval $K_{2}$ and an angle $\theta_{2} \in(0, \pi)$ so that

$$
\operatorname{Comp}_{c_{0}} f^{-\left(k_{0} j_{i}-s_{n}\right)}\left(V_{Q}\right) \subset D_{\theta_{2}}\left(K_{2}\right),
$$

where $K_{2}$ is well-inside the largest terminating interval $I_{n-1}^{i_{0}}$ in the principal nest $I_{n-1}^{0} \supset$ $I_{n-1}^{1} \supset I_{n-1}^{2} \supset \cdots$. Under these circumstances, Lemma 2.2 and the definition of successor imply that $I_{n+1}$ is a pullback of $I_{n}$ of bounded order; $\mathcal{B} \mathcal{A}\left(I_{n}\right)$ is a pullback of $I_{n}$ with order bounded from above by $2 b^{2}$, and each successor $\Gamma^{i+1}\left(\mathcal{B} \mathcal{A}\left(I_{n}\right)\right)$ is a pullback of order at most $2 b-1$ of $\Gamma^{i}\left(\mathcal{B A}\left(I_{n}\right)\right)$. Thus $I_{n+1}$ is a pullback of $I_{n}$ with order at most $2 b^{2}+5 b(2 b-1)$. For each $i \geq i_{0}$, the pullback $I_{n-1}^{i+1}$ of $I_{n-1}^{i}$ has order $b$, since $I_{n-1}^{i_{0}}$ is terminating. Therefore, increasing $k_{0}$ by $2 b+5(2 b-1)$ we can pull back $\operatorname{Comp}_{c_{0}} f^{-\left(k_{0} j_{i}-s_{n-1}\right)}\left(V_{Q}\right)$ by at most $15 b-5$ iterates of $f^{j_{i}}$ and obtain an interval $K$ that is well-inside $I_{n}$ and $\theta \in(0, \pi)$ such that $K\left(F_{Q}\right) \subset D_{\theta}(K)$. Furthermore, since $J_{i} \subset K$ and $\left|I_{n}\right| \asymp\left|I_{n+1}\right|$ we get that $|K| \asymp\left|I_{n}^{\infty}\right|$.

Now suppose that $I_{n-1}$ is terminating. Then $I_{n}=\mathcal{L}_{c_{0}}\left(\mathcal{R}\left(I_{n-1}\right)\right)$. Since $J_{i} \subsetneq I_{n}$, we have that $\mathcal{L}_{c_{0}}\left(\mathcal{R}\left(I_{n}\right)\right) \neq \mathcal{R}\left(I_{n}\right)$. Recall that $\operatorname{Comp}_{c_{0}} f^{-\left(k_{0} j_{i}-s_{n-1}\right)}\left(V_{Q}\right) \subset D_{\theta_{1}}\left(I_{n-1}\right)$. By Proposition 8.3, using three returns to $I_{n}$, see Combinatorial Remark 6, there exist $r_{n-1}<s_{n-1}$ and $\theta_{2} \in(0, \pi)$ such that $\operatorname{Comp}_{c_{0}} f^{-\left(k_{0} j_{i}-r_{n-1}\right)}\left(V_{Q}\right) \subset D_{\theta_{2}}\left(\mathcal{R}\left(I_{n-1}\right)\right)$.

Now, by Proposition 8.5, Combinatorial Remark 7, and Lemma 4.6, increasing $k_{0}$, if necessary, and pulling back by at most two more iterates of $f^{j_{i}}$, we have that there exist $s_{n}<r_{n-1}$, an interval $K$ well-inside $I_{n}$ and $\theta \in(0, \pi)$ such that Comp $_{c_{0}} f^{-\left(k_{0} j_{i}-s_{n}\right)}\left(V_{Q}\right) \subset D_{\theta}(K)$. Thus $K\left(F_{Q}\right) \subset D_{\theta}(K) \cap U_{Q}$. Furthermore, since $J_{i} \subset K$ and $\left|I_{n}\right| \asymp\left|I_{n+1}\right|$ we get that $|K| \asymp\left|I_{n}^{\infty}\right|$.

Case A.2: Unbounded geometry. Suppose that there exists $j, 0 \leq j \leq M$ such that $\left|I_{n-j}\right| /\left|I_{n-j+1}\right|>v$. Let $j_{0}, 0 \leq j_{0} \leq M$, be minimal so that $\left|I_{n-j_{0}}\right| /\left|I_{n-j_{0}+1}\right|>v$. Case A.2.1: Assume $j_{0} \geq 2$.

If $I_{n-j_{0}}$ is non-terminating, then it is easy to modify the argument in the bounded geometry case: If $I_{n-j_{0}}$ and $I_{n-j_{0}+1}$ are both non-terminating, then by Case (a) in Theorem 9.3, the first return mapping to $I_{n-j_{0}+2}$ extends to a quasiregular box mapping with 
range $D_{\pi / 2}\left(I_{n-j_{0}+2}\right)$. Since for all $0 \leq i \leq j_{0}-1$, we have that $\left|I_{n-i}\right| /\left|I_{n-i+1}\right|<v$, we argue as in Theorem 9.3 to see that there exists a qr quasi-box mapping $F: U_{Q} \rightarrow V_{Q}$ that extends the return mapping to $I_{n}$, and we can repeat the argument from the bounded geometry case to find $\theta \in(0, \pi)$ and an interval $K$, well-inside $I_{n}$, so that $K\left(F_{Q}\right) \subset D_{\theta}(K)$. Furthermore, since $J_{i} \subset K$ and $\left|I_{n}\right| \asymp\left|I_{n+1}\right|$ we get that $|K| \asymp\left|I_{n}^{\infty}\right|$. Similarly, if $I_{n-j_{0}}$ is non-terminating, and $I_{n-j_{0}+1}$ is terminating, then by Case (b) in Theorem 9.3, the return mapping from $I_{n-j_{0}+1}^{1}$ to $I_{n-j_{0}+1}$ extends to a qr polynomial-like mapping with range $D_{\pi / 2}\left(I_{n-j_{0}+1}^{2}\right)$, and we can repeat the proof of the bounded geometry case to conclude the proof in this case. So if Again, since for all $0 \leq i \leq j_{0}-1$, we have that $\left|I_{n-i}\right| /\left|I_{n-i+1}\right|<v$, we can repeat the arguments in the bounded geometry case.

Now, assume that $I_{n-j_{0}}$ is terminating. We need to consider in which case of Proposition 8.11 we are in. Let us first treat the cases that immediately reduce to the previous one:

- If we are in Case (2) of Proposition 8.11, then the first return mapping to $I_{n-j_{0}+1}^{\infty}$ extends to a qr box mapping with range $D_{\pi / 2}\left(I_{n-j_{0}+1}^{2}\right)$.

- If we are in Case (3) of Proposition 8.11, then the first return mapping to $I_{n-j_{0}+2}$ extends to a qr box mapping with range $D_{\pi / 2}\left(I_{n-j_{0}+2}\right)$.

- If we are in Case (4) of Proposition 8.11, then the first return mapping to $I_{n-j_{0}+2}^{\infty}$ extends to a qr box mapping with range $D_{\pi / 2}\left(I_{n-j_{0}+2}^{2}\right)$.

So suppose that we are in Case (1) of Proposition 8.11, which means that $\left|I_{n-j_{0}}^{\infty}\right|$ is small compared to $\left|I_{n-j_{0}}\right|$, so the first return mapping to $I_{n-j_{0}}^{\infty}$ extends to a qr box mapping with range $D_{\pi / 2}\left(I_{n-j_{0}}^{2}\right)$. Notice that we must have that $\left|I_{n-j_{0}+1}\right| \asymp\left|\mathcal{R}\left(I_{n-j_{0}}\right)\right|$ for otherwise, we would have that $I_{n-j_{0}+1}$ is $C$-nice for some $C>0$, large, which contradicts the choice of $j_{0}$.

So we have that $\left|I_{n-j_{0}+1}\right| \asymp\left|\mathcal{R}\left(I_{n-j_{0}}\right)\right|$, and $\left|I_{n-j_{0}}^{\infty}\right|$ is much smaller than $\left|I_{n-j_{0}}^{2}\right|$. Observe that if if $\left|I_{n-j_{0}+1}\right| \asymp\left|\mathcal{R}\left(I_{n-j_{0}}\right)\right|$ and $\left|I_{n-j_{0}}\right| \asymp\left|I_{n-j_{0}}^{2}\right|$, then by Lemma 3.10, $\left|I_{n-j_{0}}^{\infty}\right| \asymp\left|I_{n-j_{0}}\right|$.

Now we can repeat the argument of the bounded geometry case. This concludes the argument when $j_{0} \geq 2$.

Case A.2.2: Assume $j_{0}=1$. Then $\left|I_{n}\right|$ is small compared to $\left|I_{n-1}\right|$. Observe that by the assumptions made after the proof of the claim we must have that $I_{n-1}$ is terminating and $\left|\mathcal{R}\left(I_{n-1}\right)\right|$ is comparable to $\left|I_{n}^{\infty}\right|$. These imply that $\left|\mathcal{R}\left(I_{n-1}\right)\right|$ is much smaller than $\left|I_{n-1}\right|$. This fact, along with Lemma 3.10, implies that $\left|I_{n-1}\right|$ is much bigger than $\left|I_{n-1}^{2}\right|$. Then, we have that there exists a qr polynomial-like mapping $F: U \rightarrow V$ that extends the return mapping to $I_{n-1}^{\infty}$ with diam $(V)$ comparable to $\left|I_{n+1}\right|$. Now we can repeat the argument in the bounded geometry case to pullback one step to complete the proof.

Case A.2.3: Assume $j_{0}=0$.

There are two cases to consider depending on whether $I_{n-1}$ is terminating, either:

- $\quad I_{n-1}$ is non-terminating and $\left|I_{n-1}\right| \asymp\left|I_{n}\right|$ and $\left|I_{n}\right| /\left|I_{n+1}\right|>v$ or

- $\quad I_{n-1}$ is terminating, $\left|I_{n}^{\infty}\right| \asymp\left|\mathcal{R}\left(I_{n-1}\right)\right|$, and $\left|I_{n}\right| /\left|I_{n+1}\right|>v$.

Assume $I_{n-1}$ is non-terminating. Since $\left|I_{n}\right|$ is comparable to $\left|I_{n-1}\right|$, we can use the $j_{0} \geq 1$ cases to obtain a qr quasi-box mapping $F_{Q}^{\prime}: U_{Q}^{\prime} \rightarrow V_{Q}^{\prime}$ with $V_{Q}^{\prime}=D_{\pi / 2}\left(I_{n-j_{0}^{\prime}}\right) \cap$ $\mathbb{C}_{I_{n-1}}$ for some $j_{0}^{\prime} \geq 1$, that extends the return map to $I_{n-1}$. We obtain a quasi-box 
mapping $F_{Q}: U_{Q} \rightarrow V_{Q}$ extending the return map to $I_{n}$, with $V_{Q}=D_{\pi / 2}\left(I_{n-j_{0}^{\prime}}\right) \cap \mathbb{C}_{I_{n}}$ and $U_{Q}=\operatorname{Comp}_{c_{0}} f^{-j_{i}}\left(V_{Q}\right)$ since $F_{Q}$ is an iterate of $F_{Q}^{\prime}$. Using the argument in the cases for $j_{0} \geq 1$, we have that there exist an interval $K_{1}$ well-inside $I_{n-1}$ and $\theta_{1} \in(0, \pi)$ such that

$$
K\left(F_{Q}\right) \subset D_{\theta_{1}}\left(K_{1}\right)
$$

As in the bounded geometry case, we are going to use the argument for Proposition 8.2, to find $s_{n}^{\prime}>0, \theta_{2} \in(0, \pi)$ and $K_{2} \subset I_{n-1}^{i_{0}}$ such that $\operatorname{Comp}_{c_{0}} f^{-\left(k_{0} j_{i}-s_{n}^{\prime}\right)}\left(V_{Q}\right) \subset$ $D_{\theta_{2}}\left(K_{2}\right)$, where $i_{0} \in \mathbb{N}$ is minimal with $I_{n-1}^{i_{0}}$ terminating. However, we cannot apply Proposition 8.2 directly since we do not have that $I_{n-2}$ is comparable to $I_{n+1}$. First, notice that since $c_{0}$ is even and $I_{n-1}$ is non-terminating, Theorem 3.1 implies that there exists $\rho>0$ such that $I_{n-1}$ is $\rho$-free. Second, observe that the chain $\mathbb{G}$ is bound to enter a terminating component of the landing domain to $I_{n-1}$ containing critical point of $f$, so $k\left(I_{n-1}, \mathbb{G}\right)=\infty$. To use the same argument as in Case (ii) of Proposition 8.2, which deals with the situation when a chain has infinite combinatorial depth. Recall we are assuming $\left|I_{n-1}\right|$ is comparable to $\left|I_{n}^{\infty}\right|$. This means there exists $m \in \mathbb{N}$, depending only on the ratio $\left|I_{n-1}\right| /\left|I_{n}^{\infty}\right|$, so that $\mathcal{C}^{m}\left(\hat{\mathcal{L}}_{c}\left(I_{n-1}\right)\right)$ is a periodic interval. So the proof of Proposition 8.2 Case (ii) goes through and we obtain $\operatorname{Comp}_{c_{0}} f^{-\left(k_{0} j_{i}-s_{n}^{\prime}\right)}\left(V_{Q}\right) \subset D_{\theta_{2}}\left(K_{2}\right)$, with $s_{n}^{\prime}, \theta_{2}$ and $K_{2}$ as above. Finally, we pullback a bounded number of times by $f^{j_{i}}$, to find $\theta \in(0, \pi)$ and $K$ well-inside $I_{n}$ so that

$$
K\left(F_{Q}\right) \subset D_{\theta}(K) .
$$

Recall, we are working under the assumption that $\left|I_{n-1}\right|$ is comparable to $\left|I_{n}^{\infty}\right|$. Since $I_{n}^{\infty} \subset K$, we get that $|K| \asymp\left|I_{n}^{\infty}\right|$.

Assume now that $I_{n-1}$ is terminating and $\left|I_{n}^{\infty}\right| \asymp\left|\mathcal{R}\left(I_{n-1}\right)\right|$. Hence, $\left|I_{n}\right|$ is comparable to $\left|I_{n-1}^{\infty}\right|$. If $\left|I_{n-1}\right|$ is comparable to $\left|I_{n-1}^{\infty}\right|$, then from the cases when $j_{0} \geq 1$, we have that the return mapping to $I_{n-1}$ extends to a quasi-box mapping $F_{Q}^{\prime}: U_{Q}^{\prime} \rightarrow V_{Q}^{\prime}$, with $\left|V_{Q}^{\prime} \cap \mathbb{R}\right|$ comparable to $\left|I_{n-1}^{\infty}\right|$. Moreover we have that there exists an interval $K$ well-inside of $I_{n-1}$ and $\theta \in(0, \pi / 2)$ so that $K\left(F_{Q}^{\prime}\right) \subset D_{\theta}(K)$. On the other hand, if $\left|I_{n-1}\right| /\left|I_{n-1}^{\infty}\right|>v$, then by part (a) of the claim, applied to $I_{n-1}$, there is a qr polynomial-like mapping $F: U \rightarrow V$ that extends the return mapping to $I_{n-1}^{\infty}$ with $|V \cap \mathbb{R}| \asymp\left|I_{n-1}^{\infty}\right|$. As in the case when $I_{n-1}$ is non-terminating, we can obtain a quasibox mapping $F_{Q}: U_{Q} \rightarrow V_{Q}$ that extends the return map $f^{j_{i}}: I_{n}^{1} \rightarrow I_{n}$ as an iterate of $F_{Q}^{\prime}: U_{Q}^{\prime} \rightarrow V_{Q}^{\prime}$ or of $F: U \rightarrow V$. Moreover, there exists $\rho_{1}>0$ so that $I_{n}^{j}$ is $\rho_{1}$-free. The proof in the bounded geometry case is analogous, one just needs to consider $V \cap \mathbb{R}=I_{n-1}^{2}$. We will explain how to use the arguments for Proposition 8.3 and Proposition 8.5 to pullback through one more step in the enhanced nest. Recall we are assuming $I_{n}$ is terminating and $\left|I_{n+1}\right|$ is much smaller than $\left|I_{n}\right|$.

Since $V \cap \mathbb{R}$ is $\rho_{1}$-free, we can use the argument from Step 1 of Proposition 8.3 (compare Corollary 8.4) to find a $k_{1}, j_{i} \leq k_{1} \leq 4 j_{i}$ (see Combinatorial Remark 3), an interval $K_{1}$ well-inside $I_{n-1}^{\infty}$ and $\theta_{1} \in(0, \pi)$ so that

$$
\operatorname{Comp}_{c_{0}} f^{-\left(k_{0} j_{i}-k_{1}\right)}\left(V_{Q}\right) \subset D_{\theta_{1}}\left(K_{1}\right) .
$$

Now we argue as in Steps 2 and 3 of Proposition 8.3. It is important to notice that since $\mathcal{C}\left(\mathcal{R}\left(I_{n-1}\right)\right) \subset \mathcal{L}_{c_{0}} \mathcal{R}\left(I_{n-1}\right)=I_{n}$ is terminating, $k(X, \mathbb{G})=\infty$, where $X$ is defined as 
in Step 2 of Proposition 8.3. Because of this, we do not need to rely on having bounded geometry at a deeper level in order to control the loss of angle, since we do have control of the combinatorial depth in the parts of the chain between critical points, see Case (ii) of the proof of Proposition 8.2. Thus we obtain $k_{2}, j_{i} \leq k_{2} \leq 7 j_{i}$, see Combinatorial Remark 6, an interval $K_{2}$, well-inside $\mathcal{R}\left(I_{n-1}\right)$ and $\theta_{2} \in(0, \pi)$ such that

$$
\operatorname{Comp}_{c_{0}} f^{-\left(k_{0} j_{i}-k_{2}\right)}\left(V_{Q}\right) \subset D_{\theta_{2}}\left(K_{2}\right) .
$$

We are assuming $\mathcal{R}\left(I_{n-1}\right)$ is not a periodic interval and that $\left|I_{n}^{\infty}\right|$ is comparable to $\left|\mathcal{R}\left(I_{n-1}\right)\right|$. We use the same argument as the one used in Proposition 3.20 to prove that $\mathcal{R}\left(I_{n-1}\right)$ is $\rho^{\prime}$-free for some $\rho^{\prime}>0$. If $\mathcal{R}\left(I_{n-1}\right)$ is not periodic and terminating, then Proposition 3.20 (1) implies that there exists $\rho^{\prime}>0$ so that $\mathcal{R}\left(I_{n-1}\right)$ is $\rho^{\prime}$-free. Now, assume $\mathcal{R}\left(I_{n-1}\right)$ is non-terminating. Observe that

$$
I_{n}^{\infty} \subset \Gamma^{2}\left(\mathcal{R}\left(I_{n-1}\right)\right) \subset I_{n-1}^{\infty},
$$

and compare this with Equation (3.2). The proof of Proposition 3.20 (3) applies to this situation. Since $\left|I_{n}^{\infty}\right|$ is comparable to $\left|I_{n-1}^{\infty}\right|$, there exists $\rho^{\prime}>0$ so that $\mathcal{R}\left(I_{n-1}\right)$ is $\rho^{\prime}$-free. Finally, we repeat the argument used to prove Proposition 8.5 to obtain $k_{3}$, $0 \leq k_{3} \leq 9 j_{i}$ and $\theta_{3} \in(0, \pi)$ so that

$$
\operatorname{Comp}_{c_{0}} f^{-\left(k_{0} j_{i}-k_{3}\right)}\left(V_{Q}\right) \subset D_{\theta_{3}}\left(I_{n}\right) .
$$

Observe that, since $\mathcal{R}\left(I_{n-1}\right)$ is $\rho^{\prime}$-free there exists $\rho^{\prime \prime}>0$ so that $I_{n}$ is $\rho^{\prime \prime}$-free. So we can apply Lemma 4.6, to obtain $\theta \in(0, \pi)$ and an interval $K$ well-inside $I_{n}$ so that

$$
\operatorname{Comp}_{c_{0}} f^{-\left(k_{3}+1\right) j_{i}}\left(V_{Q}\right) \subset D_{\theta}(K),
$$

with $I_{n}^{\infty} \subset K$. Hence, $|K| \asymp\left|I_{n}^{\infty}\right|$. Since, $K\left(F_{Q}\right) \subset D_{\theta}(K) \cap U_{Q}$ the result follows.

Case B: Assume $J_{i}=I_{n}$.

In this case, $I_{n}=\mathcal{R}\left(I_{n-1}\right)=J_{i}$ is a periodic interval. Let $T_{i} \supset J_{i}$ be the largest interval so that $f^{j_{i}}$ has no critical points in $T_{i} \backslash J_{i}$, and let $S_{i}=\operatorname{Comp}_{c_{0}} f^{-j_{i}}\left(T_{i}\right)$. By Lemma 3.10, since, $\partial J_{i}=\{\alpha, \tau(\alpha)\}$, and $j_{i}=2 j_{i-1}$ where $\alpha$ is the orientation reversing fixed point closest to $c_{0}$ of $f^{j_{i-1}}$, there exists $\eta>0$ such that $(1+2 \eta) J_{i} \subset S_{i} \subset$ $(1+\eta) S_{i} \subset T_{i}$, compare [S3, Lemma 8.7]. In the case when $I_{n}=J_{i}$, the interval $K$ will always be $S_{i}$.

\section{Case B.1: Bounded geometry.}

First suppose that for no $j, 0 \leq j \leq M+1$ that $\left|I_{n-j}\right| /\left|I_{n-j+1}\right|>v$. Following the proof of the Proposition 9.3, one sees that it applies just as well to the return mapping $f^{j_{i}}: S_{i} \rightarrow T_{i}$ as it does to $f^{j_{i}}: J_{i} \rightarrow J_{i}$. This means we can construct $F_{Q}: U_{Q} \rightarrow V_{Q}$ a qr quasi-box mapping that extends the return mapping to $T_{i}$, where $V_{Q}=D_{\pi / 2}\left(I_{n-M-1}\right) \cap \mathbb{C}_{T_{i}}$. Notice that the chain $\left\{G_{i}\right\}_{i=0}^{j_{i}}$ with $G_{j_{i}}=T_{i}$ and $G_{0}=\operatorname{Comp}_{c_{0}} f^{-j_{i}}\left(T_{i}\right)=S_{i}$ has intersection multiplicity two.

Let us show that there exists $\theta_{1} \in(0, \pi / 2)$ so that $K\left(F_{Q}\right) \subset D_{\theta_{1}}\left(S_{i}\right)$. To see this, we consider two cases separately. First, suppose that for every $j, 0 \leq j \leq M, I_{n-j-1}$ is terminating, and $I_{n-j}$ is of period two under $R_{I_{n-j-1}}$. In this case, we have that each interval $I_{n-j}=J_{i-j}$, a periodic interval. We have that $\left.f^{j_{i-M}}\right|_{S_{i-M}}: S_{i-M} \rightarrow T_{i-M}$, and the chain, $\left\{\tilde{G}_{j}\right\}_{j=0}^{j_{i-M}}$ with $\tilde{G}_{j_{i-M}}=I_{i-M-1}$ and $\tilde{G}_{0}=I_{i-M-1}$ has order $2 b$. Thus by the argument in Step 1 of the proof of Proposition 8.3, we have that there exists 
$\theta \in(0, \pi)$ such that $\operatorname{Comp}_{c_{0}} f^{-j_{i-M}}\left(V_{Q}\right) \subset D_{\theta}\left(S_{i-M}\right)$. Repeating this argument for the return map to each $J_{i-j}, 0 \leq j \leq M$, we have that there exists $k_{0}, 0<k_{0} \leq M$, and $\theta_{1} \in(0, \pi / 2)$, such that

$$
\operatorname{Comp}_{c_{0}} f^{-j_{i} k_{0}}\left(V_{Q}\right) \subset D_{\theta_{1}}\left(S_{i}\right) .
$$

Thus $K\left(F_{Q}\right) \subset D_{\theta_{1}}\left(S_{i}\right)$. Alternatively, there exists $i_{0}, 0<i_{0} \leq M$ minimal so that $I_{n-i_{0}}$ is terminating, $I_{n-i_{0}}$ does not have period two under $R_{I_{n-i_{0}-1}}$ and for all $j<i_{0}$, $I_{n-j}$ does have period two under $R_{I_{n-j-1}}$. Let $F_{Q}^{\prime}: U_{Q}^{\prime} \rightarrow V_{Q}^{\prime}$ denote the quasi-box mapping that extends the return mapping to $I_{n-i_{0}}$. By Case A of this proof, we have that there exists $\theta^{\prime} \in(0, \pi / 2)$, and an interval $K^{\prime}$ with $\left|K^{\prime}\right| \asymp\left|I_{n-i_{0}}\right|$ such that such that $K\left(F_{Q}^{\prime}\right) \subset D_{\theta^{\prime}}\left(K^{\prime}\right)$. Now we can argue as in the case when all of the returns to $I_{n-j}$ were of period two under $R_{I_{n-j-1}}$ to see that there exists an angle $\theta_{1} \in(0, \pi / 2)$ such that $K\left(F_{Q}\right) \subset D_{\theta_{1}}\left(S_{i}\right)$. Thus $K\left(F_{Q}\right) \subset D_{\theta_{1}}\left(S_{i}\right) \cap D_{\pi / 2}\left(I_{n-M}\right)$.

\section{Case B.2: Unbounded geometry.}

Assume there exists $j, 0 \leq j \leq M+1$ such that $\left|I_{n-j}\right| /\left|I_{n-j+1}\right|>v$. Let $j_{0} \geq 0$ be minimal so that $\left|I_{n-j_{0}}\right| /\left|I_{n-j_{0}+1}\right|>v$.

Case B.2.1: Suppose $j_{0} \geq 2$. There exists $i_{0}, 0<i_{0} \leq j_{0}-1$ minimal so that $I_{n-i_{0}}$ is terminating, $I_{n-i_{0}}$ does not have period two under $R_{I_{n-i_{0}-1}}$ and for all $j<i_{0}, I_{n-j}$ does have period two under $R_{I_{n-j-1}}$. Let $F_{Q}^{\prime}: U_{Q}^{\prime} \rightarrow V_{Q}^{\prime}$ denote the quasi-box mapping that extends the return mapping to $I_{n-i_{0}}$, given by Case A, and repeat the proof of Case B.1 to obtain that there exists a $\theta \in(0, \pi)$ so that

$$
K\left(F_{Q}\right) \subset D_{\theta}\left(S_{i}\right) .
$$

Case B.2.2: Suppose $j_{0}=1$, or $j_{0}=0$ and $\left|I_{n-1}\right|$ is much bigger than $\left|I_{n}\right|$. Assume $j_{0}=1$. By Lemma 3.10, we know that $\left|\mathcal{R}\left(I_{n-1}\right)\right|$ is comparable to $\left|I_{n-1}^{\infty}\right|$. Furthermore, since we are assuming $\left|I_{n}^{\infty}\right|$ is comparable to $\left|\mathcal{R}\left(I_{n-1}\right)\right|$, then by Lemma 3.10, we have that $\left|I_{n-1}^{2}\right|$ is much smaller than $\left|I_{n-1}\right|$. By the claim,we can find a polynomial-like mapping $F: U \rightarrow V$ that extends the return mapping to $I_{n-1}^{\infty}$ so that $V \cap \mathbb{R}=I_{n-1}^{j}$ and $\left|I_{n-1}^{j}\right| \asymp\left|I_{n-1}^{\infty}\right|$. We obtain a quasi box mapping that extends the mapping $f^{j_{i}}: J_{i} \rightarrow J_{i}$, by setting $V_{Q}=V \cap \mathbb{C}_{T_{i}}, U_{Q}=\operatorname{Comp}_{c_{0}} f^{-j_{i}}\left(V_{Q}\right)$ and defining $F_{Q} \mid U_{Q}=F^{2}$. Since the boundary points of $T_{i}$ are critical values of $f^{j_{i}}$ and $\left|T_{i}\right|$ is comparable to $I_{n-1}^{i_{0}}$, by the argument from Step 1 of the proof of Proposition 8.3 there exists $\theta \in(0, \pi)$ such that

$$
\operatorname{Comp}_{c_{0}} f^{-j_{i}}\left(V_{Q}\right) \subset D_{\theta}\left(S_{i}\right) .
$$

Notice that this argument does not depend on $\left|I_{n}\right|$ and $\left|I_{n+1}\right|$ being comparable, so we also use it to cover the case when $j_{0}=0$ and $\left|I_{n-1}\right|$ is much bigger than $\left|I_{n}\right|$.

Case B.2.3: Suppose $j_{0}=0$ and $\left|I_{n-1}\right|$ is comparable to $\left|I_{n}\right|$. This implies that $\left|S_{i-1}\right|$ is comparable to $\left|S_{i}\right|$. We use the argument from either the bounded geometry case or the case when $j_{0} \geq 2$ with $n-1$ in place of $n$ to obtain a qr quasi-box mapping $F_{Q}^{\prime}: U_{Q}^{\prime} \rightarrow$ $V_{Q}^{\prime}$ that extends the return mapping to $I_{n-1}$, and an angle $\theta_{1} \in(0, \pi)$ such that

$$
K\left(F_{Q}^{\prime}\right) \subset D_{\theta_{1}}\left(S_{i-1}\right) .
$$

We obtain $F_{Q}: U_{Q} \rightarrow V_{Q}$ extending the first return mapping to $J_{i}$ as the second iterate of $F_{Q}^{\prime}$, just as in Case B.2.2. Now, since $\left|S_{i-1}\right|$ is comparable to $\left|S_{i}\right|$, by the argument from Step 1 of Proposition 8.3 there exists $\theta_{2} \in(0, \pi)$ so that 


$$
K\left(F_{Q}\right) \subset \operatorname{Comp}_{c_{0}} f^{-j_{i}}\left(V_{Q}\right) \subset D_{\theta_{2}}\left(S_{i}\right) .
$$

Step 2: Controlling the dilatation. Since there exist constants $C_{1}$ and $\rho^{\prime \prime}>0$ such that

$$
\operatorname{diam} J_{i} \leq \operatorname{diam} D_{\theta}(K)<C_{1}\left|J_{i}\right|,
$$

and $\left(K \backslash\left(1+2 \rho^{\prime \prime \prime}\right)^{-1} K\right)$ is disjoint from the post-critical set, there exists a constant $C_{2}>0$ such that the dilatation of $f^{j_{i}} \mid D_{\theta}(K)$ is bounded from above by $C_{2} \sum_{k=0}^{j_{i}}\left|\mathcal{L}_{f^{k}\left(c_{0}\right)}\left(J_{i}\right)\right|^{2}$.

Now, by Lemma 10.17 , we have that there exists a qr polynomial-like mapping $F_{i}: U_{i} \rightarrow V_{i}$ that extends the return mapping to $J_{i}$ such that $\bmod \left(V_{i} \backslash U_{i}\right)$ is bounded from below.

Step 3: $U_{i}$ has bounded geometry at $c_{0}$. Since $\bmod \left(V_{i} \backslash K\left(F_{i}\right)\right)$ is bounded from below and $V_{i} \supset K\left(F_{i}\right) \supset J_{i}$, it follows, for example from [M2, Proposition 4.8], that there exists $\rho>0$ so that the ball with radius $\rho$ centered at $F_{i}\left(c_{0}\right)$ is contained in $V_{i}$. If the biggest $\rho$ for which is true is small, then $V_{i}$ comes very near $J_{i}$ but still contains $J_{i}$, which means that the modulus of $V_{i} \backslash K\left(F_{i}\right)$ is necessarily small. Then since $\bmod \left(V_{i} \backslash U_{i}\right)$ is bounded from below, it follows that there also exists a $\rho^{\prime}(\rho)>0$ so that the ball with radius $\rho^{\prime}$ centered at $c_{0}$ is contained in $U_{i}$.

\section{Table of Notation and Terminology}

\begin{tabular}{|l|l|}
\hline$R_{I}$ & p. 3 \\
\hline $\mathcal{L}_{x}(I)$ & p. 3 \\
\hline$\hat{\mathcal{L}}_{x}(V)$ & p. 3 \\
\hline renormalizable & p. 4 \\
\hline central return & p. 4 \\
\hline terminating interval & p. 4 \\
\hline$J^{\infty}$ & p. 4 \\
\hline$\rho$-nice domain & p. 3 \\
\hline$\rho$-free domain & p. 3 \\
\hline$\rho$-bounded geometry & p. 3 \\
\hline$\gamma \cdot I$ & p. 17 \\
\hline Comp \\
$(K)$ & p. 17 \\
\hline$b, \underline{b}$ & p. 17 \\
\hline child & p. 19 \\
\hline persistently recurrent & p. 20 \\
\hline successor & p. 19 \\
\hline$\Gamma(I)$ & p. 20 \\
\hline central return domain & p. 19 \\
\hline non-central return domain & p. 19 \\
\hline principal nest & p. 19 \\
\hline
\end{tabular}

\begin{tabular}{|l|l|}
\hline$\hat{m}$ & p. 20 \\
\hline$\tau$ & p. 21 \\
\hline $\mathcal{R}(I)$ & p. 21 \\
\hline$Y_{i}, \tilde{\mathcal{Y}}_{\gamma}$ & p. 21 \\
\hline $\mathcal{A}(I), \mathcal{B}(I)$ & p. 21 \\
\hline $\mathcal{E}(I)$ & p. 22 \\
\hline$($ generalized $)$ enhanced nest & p. 22 \\
\hline$p_{n}$ & p. 22 \\
\hline$r(I), \hat{r}(I)$ & p. 23 \\
\hline$\rho$-nice interval & p. 24 \\
\hline$\rho$-externally free interval & p. 24 \\
\hline$\rho$-internally free interval & p. 24 \\
\hline$\rho$-free interval & p. 24 \\
\hline$\rho$-strongly nice interval & p. 24 \\
\hline well-inside, deep-inside & p. 24 \\
\hline $\mathcal{C}_{c}(I)$ & p. 29 \\
\hline$m$ & p. 29 \\
\hline Crit $(I ; J)$, Crit $\left(I ;\left\{G_{j}\right\}_{j=0}^{s}\right)$ & p. 64 \\
\hline$k\left(I,\left\{G_{j}\right\}_{j=0}^{s}\right), k(I, J), \hat{k}(I, J)$ & p. 64 \\
\hline $\mathcal{T}_{\xi}$ & p. 98 \\
\hline
\end{tabular}

Acknowledgements. This material is based upon work supported by the ERC AdG Grant No. 339523 RGDD, the National Science Foundation under Grant No. NSF 1045119 (Trevor Clark), CONACyT Grant No. 21222 (Sofia Trejo) and FAPESP Grant No. 2014/09418-0 (Sofia Trejo). Trevor Clark also thanks NSERC for their support. The authors thank the Mathematics Institute at the University of Warwick and Imperial College London for their hospitality. The authors would also like to thank Oleg Kozlovski and in particular Weixiao Shen for some very useful comments. 
Open Access This article is distributed under the terms of the Creative Commons Attribution 4.0 International License (http://creativecommons.org/licenses/by/4.0/), which permits unrestricted use, distribution, and reproduction in any medium, provided you give appropriate credit to the original author(s) and the source, provide a link to the Creative Commons license, and indicate if changes were made.

\section{References}

[AL] Avila, A., Lyubich, M.: The full renormalization horseshoe for unimodal maps of higher degree: exponential contraction along hybrid classes. Publ. Math. Inst. Hautes Études Sci. No. 114, 171-223 (2011)

[AKLS] Avila, A., Kahn, J., Lyubich, M., Shen, W.: Combinatorial rigidity for unicritical polynomials. Ann. Math. (2) 70, 783-797 (2009).

[ALS] Avila, A., Lyubich, M., Shen, W.: Avila, Parapuzzle of the Multibrot set and typical dynamics of unimodal maps. J. Eur. Math. Soc. 13(1), 27-56 (2011)

[AM1] Avila, A., Moreira, C.G.: Statistical properties of unimodal maps: the quadratic family. Ann. Math. (2) 161(2), 83-881 (2005)

[AM2] Avila, A., Moreira, C.G.: Phase-parameter relation and sharp statistical properties for general families of unimodal maps. Geometry and dynamics, pp. 1-42, Contemp. Math., 389, Amer. Math. Soc., Providence (2005)

[AIM] Astala, K., Iwaniec, T., Martin, G.: Elliptic partial differential equations and quasiconformal mappings in the plane. Princeton Mathematical Series, 48. Princeton University Press, Princeton, NJ, (2009). xviii+677 pp

[ALdM] Avila, A., Lyubich, M., de Melo, W.: Regular or stochastic dynamics in real analytic families of unimodal maps. Invent. Math. 154, 451-550 (2003)

[BSvS] Bruin, H., Shen, W., van Strien, S.: Existence of unique SRB-measures is typical for real unicritical polynomial families. Ann. Sci. École Norm. Sup. (4) 39(3), 381-414 (2006)

[BvS] Bruin, H., van Strien, S.: Monotonicity of entropy for real multimodal maps. J. AMS 28, 1-61 (2015)

[CE] Campanino, M., Epstein, H.: On the existence of Feigenbaum's fixed point. Commun. Math. Phys. 79, 261-302 (1981)

[C] Clark, T.: Regular or stochastic dynamics in families of higher-degree unimodal maps. Ergod. Theory Dyn. Syst. 34(5), 1538-1566 (2014)

[CFS] Clark, T., de Faria, E., van Strien, S.: Dynamics of asymptotically holomorphic polynomial-like maps (in preparation)

[CvS] Clark, T., van Strien, S.: Quasisymmetric rigidity in one-dimensional dynamics. Manuscript

[CvST] Clark, T., van Strien, S., Trejo, S.: Complex box bounds for real maps. arXiv:1310.8338 [math.DS]

[DH] Douady, A., Hubbard, J.: Étude dynamique des polynômes complexes, I, II. Publications Mathématiques d'Orsay, 84-02 and 85-04

[dFdM1] de Faria, E., de Melo, W.: Rigidity for critical circle maps I. J. Eur. Math. Soc. 1(4), 339-392 (1999)

[dFdM2] de Faria, E., de Melo, W.: Rigidity for critical circle maps II. J. Am. Math. Soc. 13(2), 343370 (1999)

[dFdMP] de Faria, E., de Melo, W., Pinto, A.: Global hyperbolicity of renormalization for $C^{r}$ unimodal mappings. Ann. Math. (2) 164(3), 731-824 (2006)

[Fe] Feigenbaum, M.J.: Quantitative universality for a class of nonlinear transformations. J. Stat. Phys. 19(1), 25-52 (1978)

[GdM] Guarino, P., de Melo, W.: Rigidity of smooth critical circle maps. J. Eur. Math. Soc. 19, 1729-1783

[GMdM] Guarino, P., de Melo, W.: Rigidity of critical circle maps. arXiv:1511.02792 [math.DS]

[GS] Graczyk, J., Świątek, G.: Polynomial-like mappings induced by real polynomials. In Dynamical systems and applications, volume 4 or World Sci. Ser. Appl. Anal., pp. 315-328. World Sci. Publ., River Edge (1995)

[GS2] Graczyk, J., Świątek, G.: Generic hyperbolicity in the logistic family. Ann. Math. (2) 146, 152 (1997)

[GS3] Graczyk, J., Świątek, G.: The real Fatou conjecture, Annals of Mathematics Studies, Volume 144. Princeton University Press (1998)

[GSS1] Graczyk, J., Sands, D., Świątek, G.: La dérivée schwarzienne en dynamique unimodale. (French. English, French summaries) [Schwarzian derivative in unimodal dynamics] C. R. Acad. Sci. Paris Sér. I Math. 332(4), 329-332 (2001)

[GSS2] Graczyk, J., Sands, D., Świątek, G.: Decay of geometry for unimodal maps: negative Schwarzian case. Ann. Math. (2) 161, 613-677 (2005)

[HJ] Hu, J., Jiang, Y.: The Julia set of Feigenbaum quadratic polynomial. In: Proceedings of the International Conference in Honor of Professor Liao Shantao, pp. 99-124 (1999) 
[Ka] Kahn, J.: A priori bounds for some infinitely renormalizable quadratics: I. Bounded primitive combinatorics. arXiv:math/0609045 [math.DS]

[KL1] Kahn, J., Lyubich, M.: The quasi-additivity law in conformal geometry. Ann. Math. (2) 169(2), 61593 (2009)

[KL2] Kahn, J., Lyubich, M.: Local connectivity of Julia sets for unicritical polynomials. Ann. Math. 170, 413-426 (2009)

[KL3] Kahn, J., Lyubich, M.: A priori bounds for some infinitely renormalizable quadratics. II. Decorations. Ann. Sci. Éc. Norm. Supér. (4) 41(1), 57-84 (2008)

[KL4] Kahn, J., Lyubich, M.: A priori bounds for some infinitely renormalizable quadratics. III. Molecules. Complex dynamics, pp. 229-254, A. K. Peters, Wellesley (2009)

[KT] Khanin, K., Teplinsky, A.: Renormalization horseshoe and rigidity for circle diffeomorphisms with breaks. Commun. Math. Phys. 320(2), 347-377 (2013)

[K] Kozlovski, O.: Getting rid of the negative schwarzian derivative condition. Ann. Math. (2) 152, 743762 (2000)

[KSvS] Kozlovski, O., Shen, W., van Strien, S.: Rigidity for real polynomials. Ann. Math. (2) 165, 749841 (2007)

[KSvS2] Kozlovski, O., Shen, W., van Strien, S.: Density of hyperbolicity in dimension one. Ann. Math. (2) 166, 145-182 (2007)

[KvS] Kozlovski, O., van Strien, S.: Local connectivity and quasi-conformal rigidity of non-renormalizable polynomials. Proc. Lond. Math. Soc. (3) 99(2), 275-296 (2009)

[La] Lanford, O.: A computer assisted proof of the Feigenbaum conjectures. Bull. Am. Math. Soc. (New Ser.) 6, 427-434 (1982)

[Le1] Levin, G.: Bounds for maps of an interval with one reflecting critical point. I. Fund. Math. 157, 287298 (1998)

[Le2] Levin, G.: Rigidity and non-local connectivity of Julia sets of some quadratic polynomials. Commun. Math. Phys. 304(2), 295-328 (2011)

[LvS1] Levin, G., van Strien, S.: Local connectivity of the Julia set of real polynomials. Ann. Math. (2) 147(3), 471-541 (1998)

[LvS2] Levin, G., van Strien, S.: Bounds for maps of an interval with one critical point of inflection type. Invent. Math. 141, 399-465 (2000)

[LvS3] Levin, G., van Strien, S.: Total disconnectedness of Julia sets and absense of invariant linefields for real polynomials, Géométrie complexe et systèmes dynamiques (Orsay, 1995). Astérisque No. 261, xii, 161-172 (2000)

[LiS] Li, S., Shen, W.: An improved real $C^{k}$ Koebe principle. Ergod. Theory Dyn. Syst. 30(5), 14851494 (2010)

[Ly1] Lyubich, M.: Teichmüller space of Fibonacci maps. Preprint. arXiv:math/9311213 [math.DS]

[Ly2] Lyubich, M.: Dynamics of quadratic polynomials I-II. Acta Math. 178, 185-297 (1997)

[Ly3] Lyubich, M.: Feigenbaum-Coullet-Tresser universality and Milnor's hairiness conjecture. Ann. Math. (2) 149, 319-420 (1999)

[Ly4] Lyubich, M.: Dynamics of quadratic polynomials. III. Parapuzzle and SBR measures. Géométrie complexe et systèmes dynamiques (Orsay, 1995). Astérisque No. 261 (2000), xii-xiii, pp. 173-200

[Ly5] Lyubich, M.: Almost every real quadratic map is either regular or stochastic. Ann. Math. (2) 156, 178 (2002)

[LY] Lyubich, M., Yampolsky, M.: Dynamics of quadratic polynomials: complex bounds for real maps. Ann. Inst. Fourier (Grenoble) 47(4), 1219-1255 (1997)

[MN] Martens, M., Nowicki, T.: Invariant measures for typical quadratic maps. Géométrie complexe et systèmes dynamiques (Orsay, 1995). Astérisque No. 261, xiii, pp. 239-252 (2000)

[M1] McMullen, C.: Complex dynamics and renormalization. Princeton Univ. Press, Ann. Math. Studies 135, (1994)

[M2] McMullen, C.T.: Renormalization and 3-Manifolds which Fiber over the Circle. Annals of Mathematical Studies. 142. Princeton University Press, Princeton (1996)

[dMvS] de Melo, W., van Strien, S.: One Dimensional Dynamics. Springer, Berlin (1993)

[QY] Qiu, W., Yin, Y.: Proof of the Branner-Hubbard conjecture on Cantor Julia sets. Sci. China Ser. A: Math. 52(1), 45-65 (2009)

[RvS1] Rempe, L., van Strien, S.: Absence of line fields and Mañé's theorem for nonrecurrent transcendental functions. Trans. Am. Math. Soc. 363(1), 203-228 (2011)

[RvS2] Rempe-Gillen, L., van Strien, S.: Density of hyperbolicity for classes of real transcendental entire functions and circle maps. Duke Math. J. 164(6), 1079-1137 (2015)

[S1] Shen, W.: Local connectivity of the Julia set of real polynomials (after Levin and van Strien). Sūrikaisekikenkyūsho Kōkyūroku, (988), 153-159 (1997) Complex dynamical systems and related areas (Japanese) (Kyoto, 1996)

[S2] Shen, W.: On the measurable dynamics of real rational functions. Ergod. Theory Dyn. Syst. 23, 957983 (2003) 
[S3] Shen, W.: On the metric properties of multimodal interval maps and $C^{2}$ density of Axiom A. Invent. Math. 156, 310-401 (2004)

[Sm1] Smania, D.: Complex bounds for multimodal maps: bounded combinatorics. Nonlinearity 14(5), 1311-1330 (2001)

[Sm2] Smania, D.: Phase space universality for multimodal maps. Bull. Braz. Math. Soc. New Ser. 36(2), 225-274 (2005)

[Sm3] Smania, D.: Puzzle geometry and rigidity: the Fibonacci cycle is hyperbolic. J. Am. Math. Soc. 20(3), 629-673 (2007)

[Sm4] Smania, D.: Shy shadows of infinite-dimensional partially hyperbolic invariant sets. Ergodic Theory Dyn. Syst. (2015) (to appear). arXiv:1508.07388. [math.DS]

[Sm5] Smania, D.: Solenoidal attractors with bounded combinatorics are shy (2016) arXiv:1603.06300 [math.DS]

[vSV] van Strien, S., Vargas, E.: Real bounds, ergodicity and negative Schwarzian for multimodal maps. J. Am. Math. Soc. 17(4), 749-782 (2004)

[vSV2] van Strien, S., Vargas, E.: Erratum to: "Real bounds, ergodicity and negative Schwarzian for multimodal maps" [J. Am. Math. Soc. 17(4), 749-782 (2004) (electronic); mr2083467]. J. Am. Math. Soc. 20(1)267-268 (electronic), (2007)

[Su] Sullivan, D.: Bounds, renormalization conjectures and quadratic differentials. AMS Centennial Publications II, Mathematics into the Twenty-First Century, 417-466 (1992)

[TC] Tresser, C., Coullet, P.: Itérations d'endomorphismes et groupe de renormalisation. C. R. Acad. Sci. Paris Sér. A-B 287(7), A577-A580 (1978)

[Ya] Yampolsky, M.: Complex bounds for renormalization of critical circle maps. Ergod. Theory Dyn. Syst. 19(1), 227-257 (1999)

[Ya2] Yampolsky, M.: Hyperbolicity of renormalization of critical circle maps. Publ. Math. Inst. Hautes Etudes Sci. No. 96 (2002), 1-41 (2003)

Communicated by M. Lyubich 